\title{
NMR Studies of Molecules in Liquid Crystals and Graphite
}

\author{
Mark Edward Rosen \\ (Ph.D. Thesis) \\ Materials Sciences Division \\ Lawrence Berkeley Laboratory \\ University of California \\ Berkeley, CA 94720
}

June 1992

This work was supported by the Director, Office of Energy Research, Office of Basic Energy Sciences, Materials Sciences Division, of the U.S. Department of Energy under Contract No. DE-AC03-76SF00098. 


\section{NMR Studies of Molecules in Liquid Crystals and Graphite}

Copyright (C) 1992

by

Mark Edward Rosen

The U.S. Department of Energy has the right to use this document for any purpose whatsoever including the right to reproduce all or any part thereof 


\title{
NMR Studies of Molecules in \\ Liquid Crystals and Graphite
}

by

\author{
Mark Edward Rosen
}

\begin{abstract}
NMR has proven to be a rich source of information about molecular structure in condensed phases. A particularly good example of this is magnetic dipole couplings between protons on partially-oriented molecules. These couplings are very sensitive to the distance between the coupled protons and to the orientation of their internuclear vector with respect to the applied magnetic field . This spatial dependence gives proton dipole couplings the potential to be excellent probes of molecular conformation and orientation. This potential is usually not fully realized, since couplings between protons on molecules which undergo rapid conformational changes are averaged over all molecular motions which occur. The couplings, however, can provide detailed constraints on the time-averaged conformation of a molecule that can be used as a rigorous test of models for molecular interactions.

NMR experiments to measure proton dipole couplings were performed on a series of $n$-alkanes ( $n$-hexane through $n$-decane) dissolved in nematic liquid crystals. Computer modeling of the experimental NMR spectra was done using several different models for intermolecular interactions in these systems. The model of Photinos,
\end{abstract}


et al. [1] was found to be best in describing the intermolecular interactions in these systems and can provide a statistical picture of the conformation and orientation of the alkane molecules in their partially-oriented environment. Order parameters and conformational distributions for the alkanes can be calculated from the modeling. The alkanes are found to have conformational distributions very much like those found in liquid alkanes.

Proton NMR spectra of tetrahydrofuran (THF) intercalated in two graphite intercalation compounds were also measured. Computer simulations of these spectra provide a picture of THF in the constrained environment between the graphene layers where the THF is oriented at a particular angle, can translate and rotate freely, but does not appear to pseudorotate. 


\section{Contents}

1 NMR Background 1

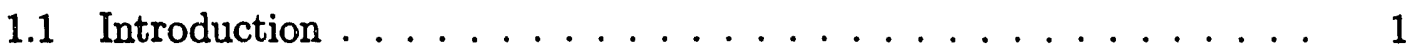

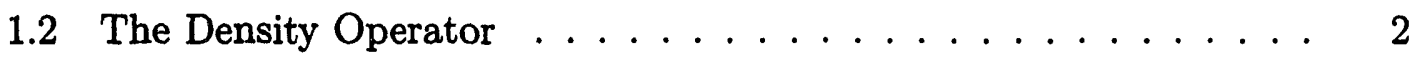

1.3 Tensors and Rotations .................. 5

1.4 NMR Hamiltonians . . . . . . . . . . . . . . . . 9

1.4.1 Zeeman Hamiltonian . . . . . . . . . . . . . 9

1.4.2 Radio Frequency Hamiltonian . . . . . . . . . . . . . 10

1.4.3 Rotating Frame Transformation . . . . . . . . . . 10

1.4.4 Chemical Shift Hamiltonian ... . . . . . . . . . 13

1.4.5 J Coupling Hamiltonian . . . . . . . . . . . . . . 16

1.4.6 Dipole Coupling Hamiltonian . . . . . . . . . . . 16

1.4.7 Quadrupole Coupling Hamiltonian . . . . . . . . . . 17

1.5 The Hamiltonian for Experiments in This Thesis . . . . . . . . . . 18

1.6 One-Dimensional NMR . . . . . . . . . . . . . . . . . . . 19

1.7 Two-Dimensional NMR . . . . . . . . . . . . . . . . 23

2 Liquid Crystals $\quad 30$

2.1 Introduction . . . . . . . . . . . . . . . 30 
2.2 Nematic Liquid Crystals . . . . . . . . . . . . . . . . . 32

2.3 Chiral Nematic (Cholesteric) Liquid Crystals . . . . . . . . . . . . . 34

2.4 Smectic Liquid Crystals . . . . . . . . . . . . . . 35

2.5 Other Mesophases . . . . . . . . . . . . . . . . 36

2.6 Order Parameters . . . . . . . . . . . . . . . . . 39

2.7 NMR in Liquid Crystals . . . . . . . . . . . . . . . . 41

3 NMR Studies of Molecular Conformation and Orientation in Liq$\begin{array}{ll}\text { uid Crystals } & 46\end{array}$

3.1 Introduction . . . . . . . . . . . . . . 46

3.2 Two-Proton Filtered COSY Experiment . . . . . . . . . . . . 48

3.3 Experimental Details . . . . . . . . . . . . . . . . 52

3.4 Experimental Dipole Couplings . . . . . . . . . . . . . 59

3.5 Modeling of Alkanes . . . . . . . . . . . . . 66

3.6 Models for $U_{\text {ext }}^{\text {aniso }}(n, \Omega) \ldots \ldots \ldots \ldots \ldots \ldots$

3.7 Results of Two-Adjustable-Parameter Modeling . . . . . . . . . 77

3.8 Results of Three-Adjustable-Parameter Modeling . . . . . . . . 87

3.9 Comparison of $n$-hexane in Liquid Crystals with $\Delta \chi>0$ and $\Delta \chi<0 \quad 94$

3.10 The Complete Set of Dipole Couplings for Hexane . . . . . . . . . 100

3.11 Further Studies with the Chord Model . . . . . . . . . . . . 100

3.12 Conclusions . . . . . . . . . . . . . . . 108

4 NMR Studies of Molecular Conformation and Orientation in Graphite $\begin{array}{ll}\text { Intercalation Compounds } & 111\end{array}$

4.1 Introduction . . . . . . . . . . . . . . . . 111

4.2 Exnorimental . . . . . . . . . . . . . . . 117 
4.3 Simulation of the NMR Spectra . . . . . . . . . . . 118

4.4 Results . . . . . . . . . . . . . . . . 127

$4.4 .1 \mathrm{Cs}(\mathrm{THF})_{1.3} \mathrm{C}_{24} \ldots \ldots \ldots \ldots \ldots \ldots$

$4.4 .2 \mathrm{~K}(\mathrm{THF})_{2.5} \mathrm{C}_{24} \ldots \ldots \ldots \ldots \ldots \ldots$

4.5 Conclusions . . . . . . . . . . . . . . . . 134

5 Tecmag Pulse Programmer Control Software 141

5.1 Hardware ......................... 141

5.1 .1 PULSkit . . . . . . . . . . . . . . . . . 142

5.1 .2 SAkit ......................... 144

5.2 TECMAG Program $\ldots \ldots \ldots \ldots \ldots . \ldots \ldots$

5.2 .1 Overview ..................... 144

5.2.2 Pulse Programming . . . . . . . . . . . . . . . . . . . 149

5.2.3 Example of Use of the TECMAG Program . . . . . . . . 153

5.2.4 More Pulse Programs . . . . . . . . . . . . . 157

5.2.5 Miscellany about the TECMAG Program . . . . . . . . . 161

$\begin{array}{ll}\text { A Software } & 166\end{array}$

A.1 Data Processing . . . . . . . . . . . . . . . 166

A.2 Simulation . . . . . . . . . . . . . . 167 


\section{List of Figures}

1.1 Euler angle definition . . . . . . . . . . . . . 7

1.2 Zeeman energy levels . . . . . . . . . . . . . . . 11

1.3 Energy levels for two coupled spins $\frac{1}{2} \ldots \ldots \ldots 24$

1.4 Peak positions and intensities for a simple COSY experiment . . . . 27

1.5 Peaks in a refocussed double-quantum-filtered COSY . . . . . . . 29

2.1 Lyotropic liquid crystals . . . . . . . . . . . . . . . . . . 31

2.2 Nematic liquid crystals . . . . . . . . . . . . . . . 33

2.3 p-pentylphenyl-2-chloro-(4-benzylbenzoyloxy)-benzoate . . . . . . 34

2.4 Cholesteric liquid crystal . . . . . . . . . . . . . . . 35

2.5 Smectic liquid crystals . . . . . . . . . . . . . . . 36

2.6 Columnar liquid crystals . . . . . . . . . . . . . . 37

2.7 Discotic liquid crystal molecules . . . . . . . . . . . . . . . 38

3.1 One-quantum spectrum of $n$-hexane . . . . . . . . . 50

3.2 Distribution of protonated isomers of $n$-hexane-d $\mathrm{d}_{81} \ldots \ldots \ldots 1$

3.3 Two-proton-filtered COSY spectrum of decane (1) . . . . . . . 55

3. Two-proton-filtered COSY spectrum of decane (2) . . . . . . . . 56

3.5 Two-proton-filtered COSY spectrum of decane (3) . . . . . . . . 57 
3.6 Numbering of protons on the alkanes . . . . . . . . . . 58

3.7 Geminal proton dipole couplings for the $n$-alkanes . . . . . . . . . 61

3.8 Proton dipole couplings to methyl protons for the $n$-alkanes . . . . 62

3.9 Other proton dipole couplings for the $n$-alkanes . . . . . . . 63

3.10 Even-odd effect in $n$-alkanes . . . . . . . . . . . . . . 64

3.11 Elastic Tube Model . . . . . . . . . . . . . . . . . . 71

3.12 Excluded Volume Model . . . . . . . . . . . . . . . . 74

3.13 Chord Model . . . . . . . . . . . . . . . 76

4.1 Pleated Layer Model for intercalation . . . . . . . . . . . . . . . . . 114

4.2 Schematic picture of $\mathrm{Cs}(\mathrm{THF})_{1.3} \mathrm{C}_{24}$ and $\mathrm{K}(\mathrm{THF})_{2.5} \mathrm{C}_{24} \ldots \ldots 115$

4.3 Temperature dependence of NMR spectrum of $\mathrm{Cs}(\mathrm{THF})_{1.3} \mathrm{C}_{24} \ldots 119$

4.4 Angular dependence of NMR spectrum of $\mathrm{Cs}(\mathrm{THF})_{1.3} \mathrm{C}_{24} \ldots \ldots$

4.5 Angular dependence of NMR spectrum of $\mathrm{K}(\mathrm{THF})_{2.5} \mathrm{C}_{24} \ldots \ldots$

4.6 Results of X-ray scattering from $\mathrm{Cs}(\mathrm{THF})_{1.3} \mathrm{C}_{24}$ and $\mathrm{K}(\mathrm{THF})_{2.5} \mathrm{C}_{24} \quad 122$

4.7 Planar reference compound for THF . . . . . . . . . . . . . . . . 124

4.8 Pseudorotation of THF . . . . . . . . . . . . 125

4.9 Simulation of proton NMR spectrum of $\mathrm{Cs}(\mathrm{THF})_{1.3} \mathrm{C}_{24} \ldots \ldots . . . \quad 129$

4.10 Simulation of proton NMR spectrum of $\mathrm{Cs}(\mathrm{THF})_{1.3} \mathrm{C}_{24} \ldots \ldots$

4.11 Simulation of proton NMR spectrum of $\mathrm{Cs}(\mathrm{THF})_{1.3} \mathrm{C}_{24} \ldots \ldots 131$

4.12 Simulation of proton NMR spectrum of $\mathrm{Cs}(\mathrm{THF})_{1.3} \mathrm{C}_{24} \ldots \ldots$

4.13 Simulation of proton NMR spectrum of $\mathrm{K}(\mathrm{THF})_{2.5} \mathrm{C}_{24} \ldots \ldots \ldots$

4.14 Simulation of proton NMR spectrum of $\mathrm{K}(\mathrm{THF})_{2.5} \mathrm{C}_{24} \ldots \ldots$

4.15 Simulation of proton NMR spectrum of $\mathrm{K}(\mathrm{THF})_{2.5} \mathrm{C}_{24} \ldots \ldots . . .137$

4.16 Simulation of proton NMR spectrum of $\mathrm{K}(\mathrm{THF})_{2.5} \mathrm{C}_{24} \ldots \ldots \ldots$ 
4.17 Simulation with free pseudorotation . . . . . . . . . . . 140

5.1 Connections between VAXStation II and Tecmag . . . . . . . . 145

5.2 Microcode word for the PULSkit pulse programmer . . . . . . . . . 146

5.3 Pulse program for one-pulse proton experiment . . . . . . . . . 150

5.4 SAkit acquisition timing . . . . . . . . . . . . . 152

5.5 Pulse program for two-dimensional proton COSY experiment . . . . 158

5.6 Pulse program for producing a rapid train of pulses . . . . . . . 159

5.7 Pulse program for TPPI multiple-quantum experiment . . . . . . 162

5.8 Pulse program for single point acquisition . . . . . . . . . 163 


\section{List of Tables}

1.1 Reduced Wigner Matrices $d_{m m^{\prime}}^{2}(\beta) \ldots \ldots \ldots \ldots \ldots$

1.2 Cartesian and spherical tensor representations of Hamiltonians . . . 14

$3.1 \mathrm{~T}, \mathrm{~T}_{N I}$, and $\mathrm{T}_{R}$ for the $n$-alkane/EK11650 samples $\ldots \ldots \ldots 53$

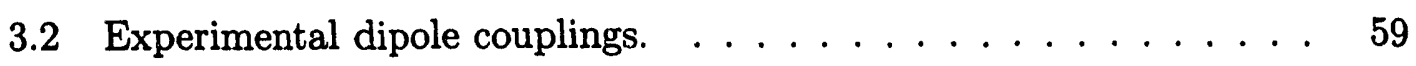

3.3 Experimental chemical shifts. . . . . . . . . . . . . . 60

3.4 Number of experimental dipole couplings for the $n$-alkanes. . . . 65

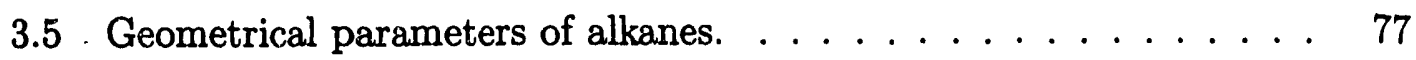

3.6 Results of two-adjustable-parameter fits . . . . . . . . . . . 79

3.7 Dipole couplings for $n$-hexane from two-parameter fits . . . . . 80

3.8 Dipole couplings for $n$-heptane from two-parameter fits . . . . . 81

3.9 Dipole couplings for $n$-octane from two-parameter fits . . . . . . 82

3.10 Dipole couplings for $n$-nonane from two-parameter fits $\ldots \ldots .83$

3.11 Dipole couplings for $n$-decane from two-parameter fits . . . . . . 84

3.12 Order parameters for $n$-alkanes from two-parameter fits . . . . 85

3.13 Conformor probabilities for $n$-alkanes from two-parameter fits $\ldots 86$

3.14 Conformer probabilities for $n$-hexane from two-parameter fits . . . 87

3.15 Results of three-adjustable-parameter fits . . . . . . . . . 88 
3.16 Dipole couplings for $n$-hexane from three-parameter fits . . . . . . 89

3.17 Dipole couplings for $n$-heptane from three-parameter fits . . . . . . 90

3.18 Dipole couplings for $n$-octane from three-parameter fits . . . . . . 91

3.19 Dipole couplings for $n$-nonane from three-parameter fits . . . . . . . 92

3.20 Dipole couplings for $n$-decane from three-parameter fits . . . . . . . 93

3.21 Order parameters for $n$-alkanes from three-parimeter fits . . . . . . 94

3.22 Conformer probabilities for $n$-alkanes from three-parameter fits . . . 95

3.23 Conformer probabilities for $n$-hexane from three-parameter fits . . . 96

3.24 Dipole couplings for $n$-hexane in EK11650 and ZLI1167 . . . . . . 97

3.25 Modeling results for $n$-hexane in EK11650 and ZLI1167 . . . . . . 99

3.26 Order parameters for $n$-hexane in EK11650 and ZLI1167 . . . . . . 99

3.27 Conformer probabilities for $n$-hexane in EK11650 and ZLI1167 . . . 99

3.28 Complete set of dipole couplings for $n$-hexane . . . . . . . . . 101

3.29 Modeling results for complete set of dipole couplings . . . . . . . . 101

3.30 Order parameters for complete set of dipole couplings . . . . . . . . 102

3.31 Conformer probabilities for complete set of dipole couplings . . . . 102

3.32 Results of four-adjustable-parameter fits . . . . . . . . . . . 103

3.33 Dipole couplings for $n$-hexane from four-parameter fits . . . . . 103

3.34 Dipole couplings for $n$-heptane from four-parameter fits . . . . . . . 104

3.35 Dipole couplings for $n$-octane from four-parameter fits . . . . . . . . 105

3.36 Dipole couplings for $n$-nonane from four-parameter fits . . . . . 106

3.37 Dipole couplings for $n$-decane from four-parameter fits . . . . . . . 107

3.38 Order parameters for $n$-alkanes from four-parameter fits . . . . . 108

3.39 Contormer probabilities for $n$-alkanes from four-parameter fits . . . 109 
3.40 Conformer probabilities for $n$-hexane from four-parameter fits . . 110

4.1 Geometrical parameters for $\mathrm{THF} \ldots \ldots . . \ldots 126$

5.1 TECMAG program commands ............... 148 


\section{Acknowledgments}

My time at Berkeley has been most enjoyable and memorable. It is rare to find someone with such a passion for science as Alex Pines. It has been a pleasure and an honor to have the opportunity to work with him these past five years.

Steve Rucker, a mentor and friend, got me through graduate school with his help, guidance, and unique sense of humor. Whether tackling the River of No Return or a recalcitrant spectrometer, he always gave $100 \%$ ("We love this stuff!"). The experiments in Chapter 3 would not have been possible without his diligence with the delta spectrometer. Claudia Schmidt laid the groundwork for the modeling in Chapter 3, and also pushed the THF/GIC project in Chapter 4 a long way towards completion. For her hard work and perseverence, I am deeply grateful. Dan Caplan was also involved in the work in Chapter 4, and his efforts are also greatly appreciated.

Brad Chmelka, Karl Mueller, and Steve Rucker were instrumental in keeping my sanity with trips to the weightroom, running in the hills, or to Cafe Roma. Softball games with Brad and the "Name Pendings" were another welcome diversion. Others who contributed to the memories I have of life in the Pines Group include Jeff Chang, John Pearson, Dave Shykind, Tom Jarvie, Bruce Black, Wrenn Wooten, Phil Grandinetti, John Harwood, Joe Zwanziger, A. J. Shaka, fellow North Dakotan Jay Baltisberger, and a host others.

I would be remiss if I didn't also thank Gerry Chingas for his innumerable 
xii

contributions both at a technical and scientific level, and Dione Carmichael for her help and guidance during my stay at Berkeley.

Finally, I would like to thank my family, my wife's family, and especially my wife, Cheryl, for their love, support, and encouragement. 
xiii

To Cheryl 


\section{Chapter 1}

\section{NMR Background}

\subsection{Introduction}

Nuclear Magnetic Resonance (NMR) has proven to be a very useful technique for elucidation of molecular structure in solution, in solids, and in partially ordered systems. In liquid-state NMR the isotropic parts of the nuclear spin Hamiltonian (chemical shift and J coupling) are observable, while the anisotropic parts are averaged to zero by the rapid, isotropic, molecular motions which occur in solution. The resuit is a high resolution, often easy-to-interpret, NMR spectrum.

Solid-state NMR provides ascess not only to the isotropic Hamiltonian, but also to the anisotropic parts of the nuclear spin Hamiltonian (anisotropic chemical shift and dipole and quadrupole couplings). The spectra obtained in solid-state NMR, however, usually contain broad, featureless lines which obscure most of the available information. Numerous pulse sequences and methods of sample reorientation have been developed which allow one to obtain narrower spectral lines and recover some of the information about the system, but the full information content of dipole and 
quadrupole couplings usually can not be easily obtained.

Partially ordered systems in which there is molecular motion - like the liquid crystals and graphite intercalation compounds discussed in this thesis - provide the best of both worlds. Rapid molecular motions in these systems result in narrow spectral lines as in liquid-state NMR, but the anisotropy of these systems allows access to both the isotropic and anisotropic parts of the Hamiltonian. The dipole and quadrupole couplings that can be obtained from these spectra provide information about molecular ordering and, in the case of the dipole couplings, molecular geometry. It is the dipole couplings which will provide the information necessary to understand the conformation and orientation of the alkane and THF molecules discussed later in this thesis.

Before pressing on with this discussion, some background on NMR necessary for discussion of the experiments in Chapters 3 and 4 will be presented. A more complete and detailed description can be found in numerous books about NMR $[2$, $3,4,5,6,7]$.

\subsection{The Density Operator}

In NMR one usually makes measurements on a macroscopic sample which contains an ensemble of many identical quantum-mechanical subsystems. To describe an NMR experiment quantum-mechanically, it is convenient for the moment to consider only one such subsystem. The state of this subsystem can be described by a particular state vector $\left|\Psi_{i}\right\rangle$. The act of measuring the state of this system involves taking an average of some observable of the subsystem, represented by a Hermitian 
operator, A. This is written :

$$
\langle\mathbf{A}\rangle_{i}=\left\langle\Psi_{i}|\mathbf{A}| \Psi_{i}\right\rangle
$$

A Hermitian operator $\mathbf{A}$ has the property $\mathbf{A}=\mathbf{A}^{\dagger}$ and has an orthonormal set of eigenvectors $\left|a_{j}\right\rangle$ each of which has a real eigenvalue $a_{j}$ :

$$
\mathbf{A}\left|a_{j}\right\rangle=a_{j}\left|a_{j}\right\rangle
$$

If $\left|\Psi_{i}\right\rangle$ is an eigenstate of $\mathbf{A}$, then the expectation value of $\mathbf{A}$ is that state's eigenvalue :

$$
\langle\mathbf{A}\rangle_{i}=a_{i}
$$

Otherwise, to compute the expectation value, it will often be convenient to expand the state $\left|\Psi_{i}\right\rangle$ in a complete set of normalized basis states (not necessarily the eigenstates of $\mathbf{A})$ :

$$
\left|\Psi_{i}\right\rangle=\sum_{m}\left|\phi_{m}\right\rangle\left\langle\phi_{m} \mid \Psi_{i}\right\rangle
$$

where the closure relation :

$$
\sum_{m}\left|\phi_{m}\right\rangle\left\langle\phi_{m}\right|=1
$$

was used. The expectation value in Equation 1.1 can now be written :

$$
\langle\mathbf{A}\rangle_{i}=\left\langle\Psi_{i}|\mathbf{A}| \Psi_{i}\right\rangle=\sum_{m} \sum_{n}\left\langle\phi_{n} \mid \Psi_{i}\right\rangle\left\langle\Psi_{i} \mid \phi_{m}\right\rangle\left\langle\phi_{m}|\mathbf{A}| \phi_{n}\right\rangle
$$

If the set of basis states, $\left|\phi_{m}\right\rangle$, was chosen to be the eigenstates of the operator $\mathbf{A}$, then this equation simplifies to :

$$
\langle\mathbf{A}\rangle_{i}=\sum_{m}\left|\left\langle\phi_{m} \mid \Psi_{i}\right\rangle\right|^{2} a_{m}
$$

so that the average is calculated by summing the eigenvalues of $\mathbf{A}\left(a_{m}\right)$ times the probability of obtaining that eigenvalue $\left(\left|\left\langle\phi_{m} \mid \Psi_{i}\right\rangle\right|^{2}\right)$. 
Since NMR measurements are made on ensembles of many identical subsystems, the next step is to consider the ensemble average of the observable $\mathbf{A}$ over all these subsystems. This adds to Equation 1.6 a probability that a subsystem is in state $\left|\Psi_{i}\right\rangle$ and sums over all possible states $\left|\Psi_{i}\right\rangle$ of the system :

$$
\langle\mathbf{A}\rangle_{e n s}=\sum_{i} \sum_{m} \sum_{n}\left\langle\phi_{n} \mid \Psi_{i}\right\rangle p_{i}\left\langle\Psi_{i} \mid \phi_{m}\right\rangle\left\langle\phi_{m}|\mathbf{A}| \phi_{n}\right\rangle
$$

where $p_{i}$ is the frobability of finding a subsystem of the ensemble in state $\left|\Psi_{i}\right\rangle$. The operator $\left|\Psi_{i}\right\rangle p_{i}\left\langle\ddot{\Psi}_{i}\right|$ is called the density operator, $\rho$. Equation 1.8 can be written :

$$
\langle\mathbf{A}\rangle_{e n s}=\sum_{m} \sum_{n}\left\langle\phi_{n}|\rho| \phi_{m}\right\rangle\left\langle\phi_{m}|\mathbf{A}| \phi_{n}\right\rangle
$$

or

$$
\langle\mathbf{A}\rangle_{e n s}=\sum_{n}\left\langle\phi_{n}|\rho \mathbf{A}| \phi_{n}\right\rangle
$$

which is the trace of $\rho \mathbf{A}$ :

$$
\langle\mathbf{A}\rangle_{\text {ens }}=\operatorname{Tr}(\rho \mathbf{A}) .
$$

I have overlooked so far that the states $\left|\Psi_{i}\right\rangle$ and therefore the density operator usually evolve in time. To do any meaningful calculations about NMR experiments, this must be considered. If the states $\left|\Psi_{i}\right\rangle$ evolve in time under the influence of a Hermitian Hamiltonian $\mathcal{H}$, the time evolution of the density operator can be described by the Liouville-von Neumann equation $[4,5,6,8]$ :

$$
\frac{d \rho}{d t}=i[\rho, \mathcal{F}]
$$

This equation can be solved for a time-independent Hamiltonian to give

$$
\rho(t)=e^{-i \mathcal{H} t} \rho(0) e^{i \mathcal{H} t}
$$


If the time-evolution of the system can be broken up into several time intervals, each governed by a different time-independent Hamiltonian, this becomes :

$$
\rho(t)=e^{-i \mathcal{H}_{n} t_{n}} e^{-i \mathcal{H}_{n-1} t_{n-1}} \cdots e^{-i \mathcal{H}_{1} t_{1}} \rho(0) e^{i \mathcal{H}_{1} t_{1}} \cdots e^{i \mathcal{H}_{n-1} t_{n-1}} e^{i \mathcal{H}_{n} t_{n}}
$$

If the initial density operator, the Hamiltonian, and the observable to be measured are known, the results of an NMR experiment can be calculated using Equations 1.11 and 1.14. The next few sections will be devoted to describing the Hamiltonians of interest, the initial density operator, and the observable to be measured. Then, in Section 1.6, Equations 1.11 and 1.14 will be used to calculate the results of a few NMR experiments. But before that, the next section will provide some background on tensors and rotations which will turn out to be quite useful in the description of NMR experiments.

\subsection{Tensors and Rotations}

I will not try to give a complete description of tensors and rotations, but will try to highlight some of the important properties and equations relating to them. For a complete discussion of tensors, see books by Rose [9] or Silver [10], and for discussion about the use of tensors in the description of NMR experiments see books by Mehring [11], Haeberlen [12], and Ernst [4].

The Hamiltonians used to describe NMR experiments can be written in terms of second-rank Cartesian tensors. For two-spin interactions the form is :

$$
\mathcal{H}=\mathbf{I} \cdot \tilde{\mathbf{A}} \cdot \mathbf{S}
$$




$$
\begin{aligned}
\mathcal{H} & =\left(I_{x}, I_{y}, I_{z}\right)\left(\begin{array}{ccc}
A_{x x} & A_{x y} & A_{x z} \\
A_{y x} & A_{y y} & A_{y z} \\
A_{z x} & A_{z y} & A_{z z}
\end{array}\right)\left(\begin{array}{c}
S_{x} \\
S_{y} \\
S_{z}
\end{array}\right) \\
\mathcal{H} & =\sum_{i, j} A_{i, j} I_{i} S_{j}
\end{aligned}
$$

Since, in the course of simulating an NMR experiment, numerous rotations often have to be performed, it is usually more convenient to write the Cartesian tensors as irreducible spherical tensors :

$$
\mathcal{H}=\sum_{k=0}^{2} \sum_{q=-k}^{k}(-1)^{q} A_{k q} T_{k-q}
$$

This separates the spatial $\left(A_{k q}\right)$ and spin $\left(T_{k-q}\right)$ parts of the Hamiltonian, so that each can be transformed separately.

Rotations of spherical tensors can be performed by specifying the Euler angles $\alpha, \beta$, and $\gamma$ (see Figure 1.1) through which to rotate the tensor, using the convention of Silver [10] for counter-clockwise rotation of a function about a fixed axis system. Such rotations can be expressed by Wigner rotation matrices :

$$
D_{p q}^{k}(\alpha, \beta, \gamma)=e^{-i \gamma p} d_{p q}^{k}(\beta) e^{-i \alpha q}
$$

where the $d_{p q}^{k}(\beta)$ are reduced Wigner rotation matrices, tabulated in Table 1.1 for $k=2[11]$. Rotations of spherical tensors can be effected by Wigner rotation matrices. The rotated tensor $T_{k q}^{\prime}$ can be written as a sum of tensors times Wigner rotation matrices :

$$
T_{k q}^{\prime}=\sum_{p=-k}^{k} T_{k p} D_{p q}^{k}(\alpha, \beta, \gamma)
$$

As a final note, Equation 1.17 can be changed into the form used by Rose [9] and Mehring [11] by exchanging angles $\alpha$ and $\gamma$ in the exponentials on the right-hand side of the equation. 

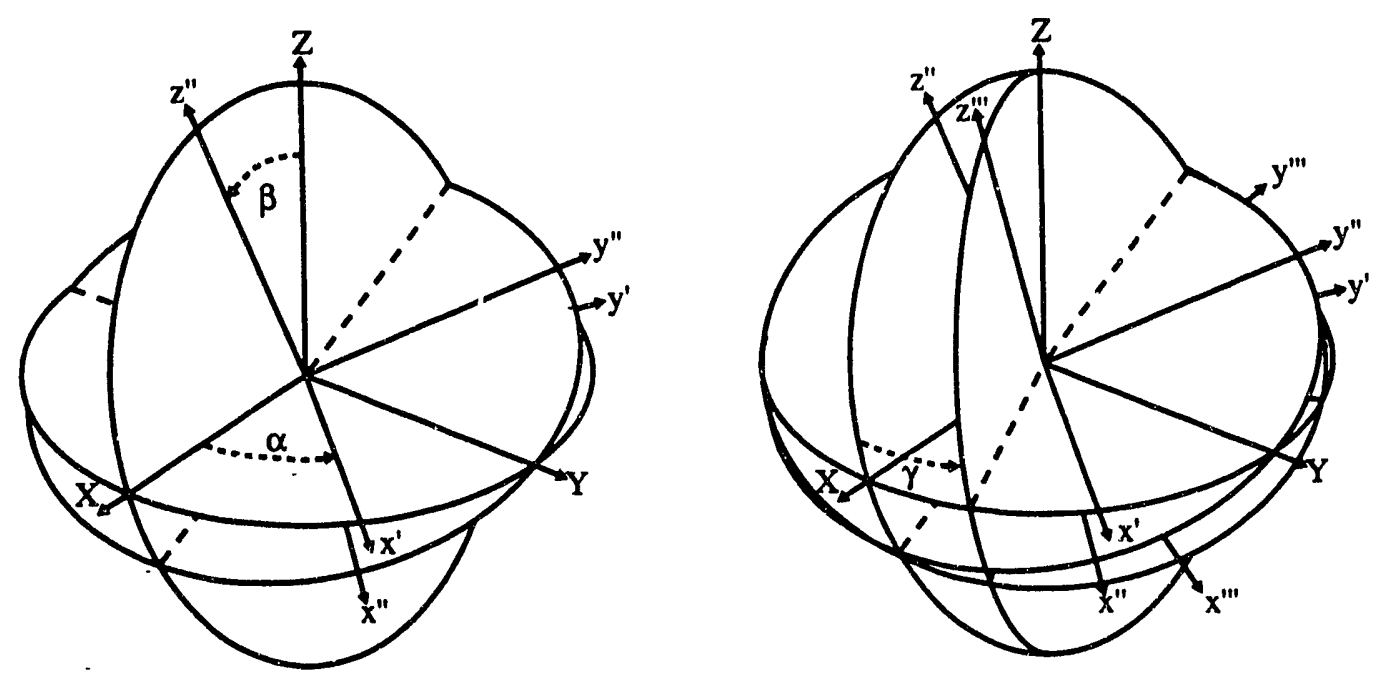

Figure 1.1: Definition of Euler angles used in this thesis. Rotations can be thought of by starting with the final and initial frames initially coincident. Then the coordinates in the initial frame are expressed in the final frame by rotating them by $\alpha$ about the final frame z-axis, by $\beta$ about the final frame $\mathrm{y}$-axis, and by $\gamma$ about the final frame $\mathrm{z}$-axis. 


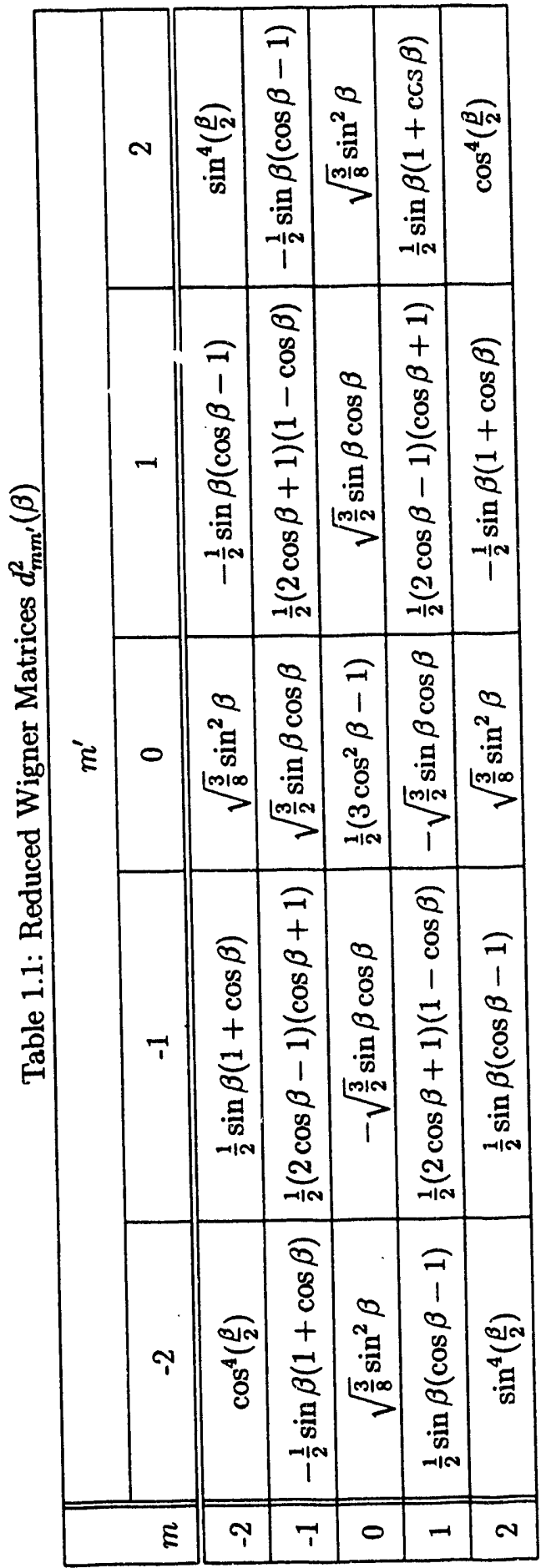




\subsection{NMR Hamiltonians}

The nuclear spin Hamiltonian can be written as a sum of external terms describing spin interactions with applied magnetic fields and internal terms describing spin interactions with local fields :

$$
\mathcal{H}=\mathcal{H}_{\text {ext }}+\mathcal{H}_{\text {int }}
$$

The external part involves two terms, the Zeeman Hamiltonian for the large applied magnetic field and the radio frequency Hamiltonian describing the applied radio frequency pulses :

$$
\mathcal{H}_{\text {ext }}=\mathcal{H}_{\mathrm{Z}}+\mathcal{H}_{\text {RF }}
$$

The internal part of the Hamiltonian is composed of several terms - chemical shift, $\mathrm{J}$ coupling, dipole coupling, and quadrupole coupling :

$$
\mathcal{H}_{\text {int }}=\mathcal{H}_{\mathrm{CS}}+\mathcal{H}_{\mathrm{J}}+\mathcal{H}_{\mathrm{D}}+\mathcal{H}_{\mathrm{Q}}
$$

Each of these Hamiltonians will be discussed in detail, starting with the external Hamiltonians.

\subsubsection{Zeeman Hamiltonian}

The principal interaction in NMR is the interaction of a nuclear spin with a large applied magnetic field. This causes the degenerate nuclear spin angular momentum energy levels to split into $2 I+1$ energy levels, each characterized by its spin angular momentum quantum number (see Figure 1.2). Written as a second-rank Cartesian tensor, this interaction has the form :

$$
\mathcal{H}_{\mathrm{Z}}=-\mathbf{I} \cdot \tilde{\mathbf{Z}} \cdot \mathbf{B}
$$


which, if the applied magnetic field is along the $z$-axis, simplifies to :

$$
\mathcal{H}_{\mathrm{Z}}=-\gamma B_{z} I_{z}
$$

where $\gamma$ is the magnetogyric ratio $\left(2.68 \times 10^{8} \mathrm{rad} \mathrm{T}^{-1} \mathrm{~s}^{-1}\right.$ for protons and 4.11 $\times 10^{7} \mathrm{rad} \mathrm{T} \mathrm{T}^{-1} \mathrm{~s}^{-1}$ for deuterons, for example), $B_{z}$ is the strength of the magnetic field (chosen along the z-axis for convenience) and $I_{z}$ is a spin angular momentum operator with eigenstates labeled by their eigenvalues, $m=-I,(-I+1), \cdots,+I$ as in Figure 1.2. The units of this and all other Hamiltonians used in this thesis are $\operatorname{rad~} \mathrm{s}^{-1}$. The Zeeman Hamiltonian can be rewritten as :

$$
\mathcal{H}_{\mathrm{Z}}=\omega_{0} I_{z}
$$

where $\omega_{0}$ is called the Larmor frequency of the nucleus.

\subsubsection{Radio Frequency Hamiltonian}

The other applied field is the oscillating radio frequency (RF) field used to induce transitions between states of the nuclear spin system. The Hamiltonian for this interaction can be written :

$$
\mathcal{H}_{\mathrm{RF}}=2 \omega_{1} \cos (\omega t+\phi) I_{x}
$$

where $\omega_{1}\left(=-\gamma \mathbf{B}_{1}\right)$ is the RF field strength, $\omega$ is the frequency of the applied RF field, and $\phi$ is its phase.

\subsubsection{Rotating Frame Transformation}

It is usually convenient to transform the Hamiltonians into a frame of reference which rotates at the frequency of the applied RF. This transformation simplifies 


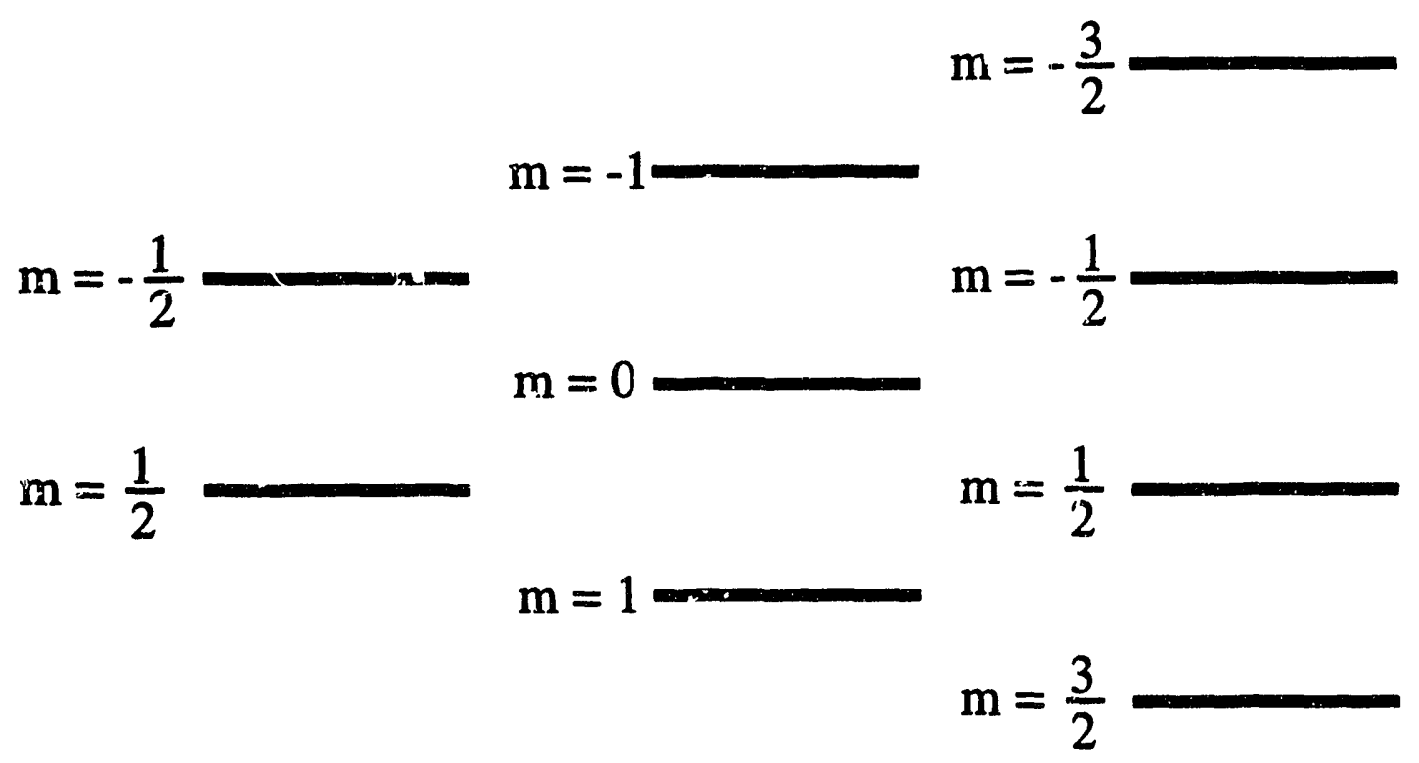

Spin $\frac{1}{2}$

Spin 1

Spin $\frac{3}{2}$

Figure 1.2: Energy levels of nuclear spins $\frac{1}{2}, 1$, and $\frac{3}{2}$ labeled by their spin angular momentum quantum numbers, $\mathrm{m}$. 
the simulation of NMR experiments and reflects how detection is done in real NMR experiments. The result of this transformation is to convert the RF Hamiltonian in Equation 1.25 to [5] :

$$
\mathcal{H}_{\mathrm{RF}}=\omega_{1}\left(\cos \phi I_{x}+\sin \phi I_{y}\right)
$$

In this frame the RF field does not oscillate, but lies at a constant orientation in the $x-y$ plane at an angle $\phi$ from the $x$-axis. The effect of the rotating frame transformation on the Zeeman Hamiltonian is to replace the Larmor frequency by a resonance offset frequency :

$$
\mathcal{H}_{\mathrm{z}}=\Delta \omega I_{z}
$$

where $\Delta \omega\left(=\omega_{0}-\omega\right)$ is the difference between the Larmor frequency and the frequency of the applied RF. In this frame tranverse magnetization appears to precess at $\Delta \omega$ rather than at $\omega_{0}$. This transfomation has the effect of removing the very large Zeeman interaction, allowing us to concentrate on the remaining internal Hamiltonians which provide the interesting chemical information about the system.

As previously discussed, the internal Hamiltonians can be expressed as a sum of irreducible spherical tensors of rank 0 through 2 :

$$
\mathcal{H}=\sum_{k=0}^{2} \sum_{q=-k}^{k}(-1)^{q} A_{k q} T_{k-q}
$$

The internal Hamiltonians are generally much smaller than the Zeeman Hamiltonian ( 0 - $50 \mathrm{kHz}$ vs. $360 \mathrm{MHz}$ for the studies in Chapter 3), so the effect of the rotatingframe transformation on these Hamiltonians is to introduce a time-dependence into all of the spin terms with $q \neq 0$, leaving $T_{00}, T_{10}$ and $T_{20}$ unaffected. The timedependent terms do not contribute to observed NMR spectra to first order [11], so they will not need to be considered in the rest of this thesis. This simplification is 
often called the high-field approximation. There are, of course, occasions when this approximation is not valid - for example, experiments in low field or experiments where $\mathcal{H}_{\mathrm{Q}}$ is very large. The term $A_{10} T_{10}$ can also be ignored since, to first order, all NMR Hamiltonians are symmetric [12] so that $A_{10}$ is zero.

The spherical tensor representations of the internal Hamiltonians are given in Table 1.2 (adapted from Reference [11]). The discussion below assumes that each Hamiltonian in this table is expressed in its principal axis system (PAS) where the second-rank Cartesian tensor is diagonal and $A_{z z}>A_{y y}>A_{x x}$. Since NMR measurements are not made in the principal axis system of the interaction, but rather in the laboratory frame, the Hamiltonians must be expressed in the lab frame. The isotropic terms, $A_{00} T_{00}$, are not affected by such a rotation, but the second-rank spatial terms become :

$$
A_{20}^{\mathrm{LAB}}=\sum_{q=-2}^{2} A_{2 q}^{\mathrm{PAS}} D_{q 0}^{2}(\alpha, \beta, \gamma) .
$$

This involves rotating the spatial part of the Hamiltonian from its PAS to the lab frame. Since $A_{2 m}^{\mathrm{PAS}}$ is diagonal in the Cartesian representation, only the terms $A_{20}^{\mathrm{PAS}}$ and $A_{2 \pm 2}^{\mathrm{PAS}}$ are non-zero. This puts the internal Hamiltonians into the form:

$$
\mathcal{H}^{\mathrm{LAB}}=\left[A_{20}^{\mathrm{PAS}} D_{00}^{2}(\alpha, \beta, \gamma)+A_{22}^{\mathrm{PAS}} D_{20}^{2}(\alpha, \beta, \gamma)+A_{2-2}^{\mathrm{PAS}} D_{-20}^{2}(\alpha, \beta, \gamma)\right] T_{20}
$$

\subsubsection{Chemical Shift Hamiltonian}

Nuclei aren't isolated, but instead are surrounded by electrons. These electrons shield the nucleus to some extent from the applied magnetic field, causing a shift of the resonance frequency of the nucleus. In Cartesian form the chemical shift 
Table 1.2: Relationship between Cartesian and spherical tensor representations of nuclear spin interactions for the internal Hamiltonians.

\begin{tabular}{|c|c|}
\hline \multicolumn{2}{|c|}{ Cartesian Spatial Tensors $A_{\alpha \beta}(\alpha, \beta=x, y, z)$} \\
\hline Chemical Shift & $\gamma \sigma_{\alpha \beta}$ \\
\hline Dipole Coupling & $\mathrm{D}_{\alpha \beta}^{12}=-\frac{\gamma_{1} \gamma_{2} h}{\pi r_{12}^{3}} \frac{1}{2}\left[\frac{3 r_{\alpha, 12} r_{\beta, 12}}{r_{12}^{2}}-1\right]$ \\
\hline $\mathrm{J}$ Coupling & $\mathrm{J}_{\alpha \beta}^{12}=2 \pi J \frac{1}{2}\left[\frac{3 r_{\alpha, 12} r_{\beta, 12}}{r_{12}^{2}}-1\right]$ \\
\hline Quadrupole Coupling & $\mathrm{Q}_{\alpha \beta}=\frac{e Q}{2 I(2 I-1)} V_{\alpha \beta}$ \\
\hline
\end{tabular}

\begin{tabular}{|c||c|}
\hline \multicolumn{2}{|c|}{ Spatial Tensors } \\
\hline$A_{00}$ & $-\frac{1}{\sqrt{3}}\left(A_{x x}+A_{y y}+A_{z z}\right)$ \\
$A_{10}$ & $-\frac{i}{\sqrt{2}}\left(A_{x y}-A_{y x}\right)$ \\
$A_{1 \pm 1}$ & $-\frac{1}{2}\left[A_{z x}-A_{x z} \pm i\left(A_{z y}-A_{y z}\right)\right]$ \\
$A_{20}$ & $\frac{1}{\sqrt{6}}\left[3 A_{z z}-\left(A_{x x}+A_{y y}+A_{z z}\right)\right]$ \\
$A_{2 \pm 1}$ & $\mp \frac{1}{2}\left[A_{x z}+A_{z x} \pm i\left(A_{y z}+A_{z y}\right)\right]$ \\
$A_{2 \pm 2}$ & $\frac{1}{2}\left[A_{x x}-A_{y y} \pm i\left(A_{x y}+A_{y x}\right)\right]$ \\
\hline
\end{tabular}

\begin{tabular}{|c|c|c|c|c|}
\hline \multicolumn{5}{|c|}{ Spin Tensors } \\
\hline & Chemical Shift & J Coupling & Dipole Coupling & Quadrupole Coupling \\
\hline$T_{00}$ & $-\frac{1}{\sqrt{3}} I_{z} B_{z}$ & $-\frac{1}{\sqrt{3}} \mathbf{I} \cdot \mathbf{S}$ & 0 & 0 \\
\hline$T_{10}$ & 0 & $-\frac{1}{2 \sqrt{2}}\left(I^{+} S^{-}-I^{-} S^{+}\right)$ & 0 & 0 \\
\hline$T_{1 \pm 1}$ & $-\frac{1}{2} I^{ \pm} B_{z}$ & $\frac{1}{2}\left(I_{z} S^{ \pm}-I^{ \pm} S_{z}\right)$ & 0 & 0 \\
\hline$T_{20}$ & $\sqrt{\frac{2}{3}} I_{z} B_{z}$ & $\frac{1}{\sqrt{6}}\left(3 I_{z} S_{z}-\mathbf{I} \cdot \mathbf{S}\right)$ & $\frac{1}{\sqrt{6}}\left(3 I_{z} S_{z}-\mathbf{I} \cdot \mathbf{S}\right)$ & $\frac{1}{\sqrt{6}}\left[3 I_{z}^{2}-I(I+1)\right]$ \\
\hline$T_{2 \pm 1}$ & $\mp \frac{1}{2} I^{ \pm} B_{z}$ & $\mp \frac{1}{2}\left(I_{z} S^{ \pm}+I^{ \pm} S_{z}\right)$ & $\mp \frac{1}{2}\left(I_{z} S^{ \pm}+I^{ \pm} S_{z}\right)$ & $\mp \frac{1}{2}\left(I_{z} I^{ \pm}+I^{ \pm} I_{z}\right)$ \\
\hline$T_{2 \pm 2}$ & 0 & $\frac{1}{2} I^{ \pm} S^{ \pm}$ & $\frac{1}{2} I^{ \pm} S^{ \pm}$ & $\frac{1}{2} I^{ \pm} I^{ \pm}$ \\
\hline
\end{tabular}


Hamiltonian can be written :

$$
\mathcal{H}_{\mathrm{CS}}=\mathbf{I} \cdot \tilde{\sigma} \cdot \mathbf{B}_{0}
$$

The tensor $\tilde{\sigma}$ can be separated into isotropic and anisotropic parts using spherical tensors. The isotropic part :

$$
\begin{gathered}
\mathcal{H}_{\mathrm{CS}(\mathrm{iso})}=A_{00} T_{00}=\gamma B_{z} \frac{1}{3}\left(\sigma_{x x}+\sigma_{y y}+\sigma_{z z}\right) I_{z} \\
\mathcal{H}_{\mathrm{CS}(\text { iso })}=-\omega_{0} \sigma_{\text {iso }} I_{z}
\end{gathered}
$$

has the same dependence on the large applied magnetic field as the Zeeman Hamiltonian and can be combined with $\mathcal{H}_{\mathrm{z}}$ in Equation 1.24 :

$$
\mathcal{H}_{\mathrm{z}+\mathrm{CS}(\mathrm{iso})}=\omega_{0}\left(1-\sigma_{\mathrm{iso}}\right) I_{z}
$$

Equation 1.27 for the rotating-frame Zeeman Hamiltonian must also be modified :

$$
\mathcal{H}_{\mathrm{z}+\mathrm{CS}(\mathrm{iso})}=\Delta \omega I_{z}
$$

where $\Delta \omega=\omega_{0}\left(1-\sigma_{i s o}\right)-\omega$.

The anisotropic part of the chemical shift is :

$$
\begin{aligned}
\mathcal{H}_{\mathrm{CS}(\text { aniso })}^{\mathrm{LAB}}= & {\left[A_{20}^{\mathrm{PAS}} D_{00}^{2}(\alpha, \beta, \gamma)+A_{22}^{\mathrm{PAS}} D_{20}^{2}(\alpha, \beta, \gamma)+A_{2-2}^{\mathrm{PAS}} D_{-20}^{2}(\alpha, \beta, \gamma)\right] T_{20} } \\
\mathcal{H}_{\mathrm{CS}(\text { aniso })}^{\mathrm{LAB}}= & -\frac{1}{3} \omega_{0}\left[\sigma_{z z}-\frac{1}{2}\left(\sigma_{x x}+\sigma_{y y}\right)\right]\left(3 \cos ^{2} \beta-1\right) I_{z} \\
& -\frac{1}{2} \omega_{0}\left[\left(\sigma_{x x}-\sigma_{y y}\right) \sin ^{2} \beta \cos 2 \alpha\right] I_{z}
\end{aligned}
$$

If we define $\Delta \sigma=\sigma_{z z}-\frac{1}{2}\left(\sigma_{x x}+\sigma_{y y}\right)$ as the chemical shift anisotropy and $\eta=$ $\frac{3}{2} \frac{\sigma_{x x}-\sigma_{y y}}{\Delta \sigma}$ as the asymmetry of the chemical shift, then this becomes :

$$
\mathcal{H}_{\mathrm{CS}(\text { aniso })}^{\mathrm{LAB}}=-\frac{1}{3} \omega_{0} \Delta \sigma\left[\left(3 \cos ^{2} \beta-1\right)+\eta \sin ^{2} \beta \cos 2 \alpha\right] I_{z}
$$

where $\alpha$ and $\beta$ are the Euler angles that relate the PAS of the chemical shift tensor and the lab frame with the applied magnetic field along the z-axis. 


\subsubsection{J Coupling Hamiltonian}

The $\mathrm{J}$ Coupling is an indirect interaction of nuclear spins through their effect on electrons in the chemical bonds that separate the coupled nuclei. As can be seen in Table 1.2, the interaction has both isotropic and anisotropic components. In proton NMR the anisotropic component has been found to be negligible (though not in fluorine NMR) [13] and will not be discussed further, except to point out that the second-rank part of the $\mathrm{J}$ coupling has the same form as the dipole coupling to be discussed in the next section. This means that even if the anisotropic $\mathrm{J}$ coupling is not negligible, it is difficult to separate the contributions of the anisotropic $\mathrm{J}$ coupling and the dipole coupling in experimental spectra.

The isotropic part of the $\mathrm{J}$ coupling has the form :

$$
\mathcal{H}_{\mathrm{J}}=A_{00} T_{00}=2 \pi \mathrm{JI}_{1} \cdot \mathbf{I}_{2}
$$

The values of proton-proton $\mathrm{J}$ couplings observed in liquid-state proton NMR are typically less than $20 \mathrm{~Hz}$.

\subsubsection{Dipole Coupling Hamiltonian}

The dipole coupling is the internal Hamiltonian of most interest in this thesis. It has an anisotropic part, but no isotropic part. This means that it is not observed in liquid-state NMR spectra where rapid tumbling of molecules averages out the orientational dependence of the couplings. Like in the case of the anisotropic chemical shift, the dipole coupling tensor in Table 1.2 is expressed in the PAS of the interaction - that is, with $\mathrm{D}_{z z}$ along the internuclear vector between the two coupled spins. Since measurements are done in the lab frame, not the PAS, we must rotate 
the tensor into this frame :

$$
\mathcal{H}_{\mathrm{D}}^{\mathrm{LAB}}=\left[A_{20}^{\mathrm{PAS}} D_{00}^{2}(\alpha, \beta, \gamma)+A_{22}^{\mathrm{PAS}} D_{20}^{2}(\alpha, \beta, \gamma)+A_{2-2}^{\mathrm{PAS}} D_{-20}^{2}(\alpha, \beta, \gamma)\right] T_{20}
$$

The dipole coupling is traceless and, in its PAS, it is axially symmetric $\left(D_{x x}=D_{y y}=\right.$ $\left.-\frac{1}{2} \mathrm{D}_{z z}=\frac{\gamma_{1} \gamma_{2} h}{2 \pi r_{12}^{3}}\right)$, so that $A_{22}^{\text {PAS }}=A_{2-2}^{\text {PAS }}=0$ and the dipole coupling Hamiltonian for two coupled nuclei simplifies to :

$$
\mathcal{H}_{\mathrm{D}}^{\mathrm{LAB}}=-\frac{\gamma_{1} \gamma_{2} h}{2 \pi r_{12}^{3}} \frac{1}{2}\left(3 \cos ^{2} \beta-1\right)\left(3 I_{z 1} I_{z 2}-I_{1} \cdot \mathbf{I}_{2}\right)
$$

With the relation $I^{ \pm}=I_{x} \pm i I_{y}$ this becomes :

$$
\mathcal{H}_{\mathrm{D}}^{\mathrm{LAB}}=-\frac{\gamma_{1} \gamma_{2} h}{2 \pi r_{12}^{3}}\left(3 \cos ^{2} \beta-1\right)\left[I_{z 1} I_{z 2}-\frac{1}{4}\left(I_{1}^{+} I_{2}^{-}+I_{1}^{-} I_{2}^{+}\right)\right]
$$

where $\beta$ is the angle between the internuclear vector and the applied magnetic field.

\subsubsection{Quadrupole Coupling Hamiltonian}

A quadrupole coupling only occurs when a nucleus is spin 1 or greater. It is due to the interaction of a non-spherical nuclear charge distribution with external electric field gradients. The quadrupole coupling (like the dipole coupling) is traceless, so there is no isotropic component which can be observed in liquid-state NMR. Unlike the dipole coupling, a quadrupole coupling does not have to be axially symmetric $\left(A_{22}^{\mathrm{PAS}}=A_{2-2}^{\mathrm{PAS}} \neq 0\right)$, so in the lab frame it can be written :

$$
\mathcal{H}_{\mathrm{Q}}^{\mathrm{LAB}}=\left[A_{20}^{\mathrm{PAS}} D_{00}^{2}(\alpha, \beta, \gamma)+A_{22}^{\mathrm{PAS}} D_{20}^{2}(\alpha, \beta, \gamma)+A_{2-2}^{\mathrm{PAS}} D_{-20}^{2}(\alpha, \beta, \gamma)\right] T_{20}
$$

which becomes :

$\mathcal{H}_{\mathrm{Q}}^{\mathrm{LAB}}=\frac{e Q}{8 I(2 I-1)}\left[V_{z z}\left(3 \cos ^{2} \beta-1\right)+\left(V_{x x}-V_{y y}\right) \sin ^{2} \beta \cos 2 \alpha\right]\left[3 I_{z}^{2}-I(I+1)\right]$ 
where $I$ is the spin of the nucleus, $e$ is the charge of an electron, $Q$ is the quadrupole coupling constant for the nucleus, and $V_{\alpha \alpha}$ are the Cartesian components of the

electric field gradient tensor at the nucleus. If $\eta$ is defined as $\frac{\left(V_{x x}-V_{y y}\right)}{V_{z z}}$ then this becomes :

$$
\mathcal{H}_{\mathrm{Q}}^{\mathrm{LAB}}=\frac{e Q V_{z z}}{8 I(2 I-1)}\left[\left(3 \cos ^{2} \beta-1\right)+\eta \sin ^{2} \beta \cos 2 \alpha\right]\left[3 I_{z}^{2}-I(I+1)\right]
$$

where $\alpha$ and $\beta$ are the Euler angles that relate the PAS of the quadrupole coupling tensor and the lab frame with the applied magnetic field along the z-axis.

\subsection{The Hamiltonian for Experiments in This}

\section{Thesis}

The various Hamiltonians which are accessible in NMR experiments on different types of systems were discussed in the previous sections. The experiments in this thesis are proton NMR experiments on $n$-alkanes dissolved in liquid crystals and on tetrahydrofuran (THF) in graphite intercalation compounds. In these systems the molecules of interest undergo rapid, anisotropic motion, so that NMR experiments, which are on a time scale much slower than the molecular motions, result in partially averaged spectra. One consequence of this is that dipole couplings between protons on different molecules are averaged to zero. This simplifies the simulation of the spectra of these systems because the signal observed appears to come from an ensemble of identical, isolated molecules.

The Hamiltonian for these systems is :

$$
\mathcal{H}=\mathcal{H}_{\mathrm{Z}}+\mathcal{H}_{\mathrm{CS}}+\mathcal{H}_{\mathrm{J}}+\mathcal{H}_{\mathrm{D}}
$$


The nature of the partially-averaged spectra that are observed leads to some simplifications in the Hamiltonians. First, the anisotropic portions of the Hamiltonian are partially averaged, so the observed chemical shift results from the isotropic chemical shift plus the average value of the anisotropic chemical shift and will be written :

$$
\mathcal{H}_{\mathrm{CS}}=-\omega_{0}\langle\sigma\rangle I_{z}
$$

Now $\Delta \omega$ in the rotating frame Hamiltonian in Equation 1.35 becomes $\langle\Delta \omega\rangle=$ $\omega_{0}(1-\langle\sigma\rangle)-\omega$. Second, the the J coupling is ignored. This is done because the resolution of the spectra is not sufficient to observe splittings and line shifts due to J couplings. Finally, the dipole couplings are also averaged over the molecular motions that occur. The total evolution Hamiltonian consists of a resonance offset term containing chemical shift and the partially averaged dipole coupling Hamiltonian :

$$
\mathcal{H}=\sum_{i}\langle\Delta \omega\rangle_{i} I_{z i}+\sum_{i>j}-\frac{\gamma_{i} \gamma_{j} h}{2 \pi}\left\langle\frac{1}{r_{i j}^{3}}\left(3 \cos ^{2} \beta_{i j}-1\right)\right\rangle\left[I_{z i} I_{z j}-\frac{1}{4}\left(I_{i}^{+} I_{j}^{-}+I_{i}^{-} I_{j}^{+}\right)\right]
$$

where $i$ and $j$ are are summed over all the protons on the molecule of interest. The other Hamiltonian which will be of interest between evolution periods is the RF Hamiltonian in Equation 1.26 describing the effect of the RF pulses.

\subsection{One-Dimensional NMR}

Now that the Hamiltonian governing the systems in this thesis is known, we need to specify the initial density operator and the observable before calculating NMR spectra. At equilibrium the density operator is diagonal [5] with the diagonal elements representing populations of the states. The states are populated at equilibrium as 
prescribed by the Boltzman distribution :

$$
p_{i} \propto e^{-\beta_{0} E_{i}}
$$

where $\beta_{0}=\frac{1}{k T}$ and $E_{i}$ is the energy of state $i$. The equilibrium density operator can be written :

$$
\rho_{\mathrm{eq}}=p_{i} \propto e^{-\beta_{0} \omega_{0} I_{z}}
$$

where the Zeeman energy is $\omega_{0} I_{z}$. Since energies in NMR are much less than $k T$ at room temperature, it is possible to expand the exponential in a series and truncate it :

$$
\rho_{\mathrm{eq}}=1-\beta_{0} \omega_{0} I_{z}
$$

The constant term doesn't evolve in time and will be dropped, as will the constants $\beta_{0} \omega_{0}$, leaving the reduced equilibrium density operator :

$$
\rho_{\mathrm{eq}}=I_{z}
$$

This will be the starting density operator for the calculations to follow.

The remaining issue is the observable in an NMR experiment. In NMR experiments, detection is done with the same RF coil used to supply the RF pulses. The coil simultaneously detects the real $\left(I_{x}\right)$ and imaginary $\left(I_{y}\right)$ parts of the signal. This is called quadrature detection and will be represented by the observable $I^{+}=I_{x}+i I_{y}$. Now everything needed to calculate the results of an NMR experiment has been discussed : The initial density operator (Equation 1.52), the equation for time evolution of the density operator (Equation 1.14), the evolution (Equation 1.48) and RF (Equation 1.26) Hamiltonians, and the equation for the expectation value of an observable (Equation 1.11). 
The simplest type of NMR experiment involves the application of a $\left(\frac{\pi}{2}\right)_{y}$ pulse to the spin system and detection of the resulting signal. The observed signal in such an experiment can be written :

$$
S(t)=\left\langle I^{+}\right\rangle=\operatorname{Tr}\left(\rho I^{+}\right)=\operatorname{Tr}\left(e^{-i \mathcal{H} t} e^{-i \frac{\pi}{2} I_{\nu}} \rho_{\mathrm{eq}} e^{i \frac{\pi}{2} I_{v}} e^{i \mathcal{H} t} I^{+}\right)
$$

where the RF Hamiltonian is expressed in terms of the tip angle $\theta=\omega_{1} \tau=\frac{\pi}{2}$ of the pulse. The density operator immediately after the pulse can be calculated :

$$
\rho(0)=e^{-i \frac{\pi}{2} I_{\nu}} I_{z} e^{i \frac{\pi}{2} I_{y}}=I_{x}
$$

Then

$$
S(t)=\operatorname{Tr}\left(e^{-i \mathcal{H} t} I_{x} e^{i \mathcal{H} t} I^{+}\right)
$$

or using the properties of the trace [8] :

$$
S(t)=\operatorname{Tr}\left(I_{x} e^{i \mathcal{H} t} I^{+} e^{-i \mathcal{H} t}\right)
$$

For computer calculation of the observed signal, a basis set must be chosen for evaluation of the trace. The most convenient basis will be the basis of eigenstates of the Hamiltonian, written $|i\rangle$. The signal is :

$$
\begin{gathered}
S(t)=\sum_{i}\left\langle i\left|I_{x} e^{i \mathcal{H} t} I^{+} e^{-i \mathcal{H} t}\right| i\right\rangle \\
S(t)=\sum_{i, j}\left\langle i\left|I_{x} e^{i \mathcal{H} t}\right| j\right\rangle\left\langle j\left|I^{+} e^{-i \mathcal{H} t}\right| i\right\rangle \\
S(t)=\sum_{i, j} e^{i\left(\omega_{j}-\omega_{i}\right) t}\left\langle i\left|I_{x}\right| j\right\rangle\left\langle j\left|I^{+}\right| i\right\rangle
\end{gathered}
$$

where $\omega_{i}$ and $\omega_{j}$ are the eigenvalues of the Hamiltonian and $\left(\omega_{j}-\omega_{i}\right)$ are the observed transition frequencies. The difference between the diagonal elements of the Hamiltonian matrix $\left(\omega_{j}-\omega_{i}\right)$ gives the observed transition frequencies, and the 
product $\left\langle i\left|I_{x}\right| j\right\rangle\left\langle j\left|I^{+}\right| i\right\rangle$ gives the relative amplitude of the signal at this frequency. For computational purposes the Hamiltonian and the operators $I^{+}$and $I_{x}$ must be written in the basis of the eigenstates of the Hamiltonian. To do this one usually first constructs the operators in the product basis (the basis formed by multiplying the Zeeman states of the individual nuclei) and then diagonalizes the Hamiltonian matrix. Mathematically this is :

$$
D=U^{\dagger} \mathcal{H U}
$$

where $D$ is the diagonal matrix and $U$ is the transformation matrix which takes an operator from the product basis to the basis where the Hamiltonian is diagonal. Then the other operators can be transformed to this basis by matrix multiplication :

$$
\begin{array}{r}
I_{x}^{\prime}=U^{\dagger} I_{x} U \\
I^{+\prime}=U^{\dagger} I^{+} U .
\end{array}
$$

The only thing left to make the simulation look like a real spectrum (besides noise) is to mimic the effect of relaxation. This is done by assuming a Lorentzian line shape and multiplying the time-domain signal by an exponential $e^{-\frac{t}{T_{2}}}$ where $T_{2}$ is the characteristic spin-spin relaxation time of the nuclei. The lines in the spectrum will have a width at half height of $\frac{1}{\pi T_{2}} \mathrm{~Hz}$. Alternatively, each line in the frequency domain can be broadened by weighting it with a Lorentzian of width at half height of $\frac{1}{\pi T_{2}} \mathrm{~Hz}$.

Chapter 3 will consider systems of two isolated spins $\frac{1}{2}$. For this simple case, it is possible to analytically solve for eigenvalues and eigenvectors of the Hamiltonian. These are shown in the energy level diagram in Figure 1.3. From this diagram it 
can be seen that the observable one-quantum transitions have frequencies :

$$
\begin{aligned}
& \omega_{21}=\bar{\omega}-\frac{\mathrm{D}}{2}+\frac{1}{2} \sqrt{(\delta \omega)^{2}+\frac{\mathrm{D}^{2}}{4}} \\
& \omega_{31}=\bar{\omega}-\frac{\mathrm{D}}{2}-\frac{1}{2} \sqrt{(\delta \omega)^{2}+\frac{\mathrm{D}^{2}}{4}} \\
& \omega_{42}=\bar{\omega}+\frac{\mathrm{D}}{2}-\frac{1}{2} \sqrt{(\delta \omega)^{2}+\frac{\mathrm{D}^{2}}{4}} \\
& \omega_{43}=\bar{\omega}+\frac{\mathrm{D}}{2}+\frac{1}{2} \sqrt{(\delta \omega)^{2}+\frac{\mathrm{D}^{2}}{4}} .
\end{aligned}
$$

The intensities of the peaks at these frequencies in a simple one-pulse experiment are given by $\left\langle i\left|I_{x}\right| j\right\rangle\left\langle j\left|I^{+}\right| i\right\rangle$ and are :

$$
\begin{aligned}
& I_{21}=1+\sin 2 \theta \\
& I_{31}=1-\sin 2 \theta \\
& I_{42}=1+\sin 2 \theta \\
& I_{43}=1-\sin 2 \theta
\end{aligned}
$$

with $\theta$ defined in Figure 1.3. If $|\mathrm{D}|>>|\delta \omega|$, as is often the case, then (assuming the sign of $\delta \omega$ is positive) depending on the sign of $\mathrm{D}$, either the pair of lines at $\omega_{21}$ and $\omega_{42}$ or $\omega_{31}$ and $\omega_{43}$ will have intensity close to zero, leaving only two lines in the spectrum at $\bar{\omega} \pm\left(\frac{|D|}{2}+\frac{1}{2} \sqrt{(\delta \omega)^{2}+\frac{D^{2}}{4}}\right)$. The splitting in the spectrum will then be $\left(|D|+\sqrt{(\delta \omega)^{2}+\frac{D^{2}}{4}}\right)$. If the difference in chemical shift between the two nuclei, $\delta \omega$, is zero, the splitting will be $\frac{3}{2}|\mathrm{D}|$.

\subsection{Two-Dimensional NMR}

Two-dimensional NMR was first proposed by Jeener in 1972 [14]. The primary benefits of two-dimensional NMR are the simplification of crowded one-dimensional 


$$
\begin{aligned}
& \frac{E_{4}=\bar{\omega}+\frac{D}{4}}{\left|\phi_{4}\right\rangle=|\beta \beta\rangle} \\
& E_{2}=-\frac{D}{4}+\frac{1}{2} \sqrt{(\delta \omega)^{2}+\frac{D^{2}}{4}} \\
& \left|\phi_{2}\right\rangle=\cos \theta|\alpha \beta\rangle+\sin \theta|\beta \alpha\rangle \\
& E_{3}=-\frac{D}{4}-\frac{1}{2} \sqrt{(\delta \omega)^{2}+\frac{D^{2}}{4}} \\
& \left|\phi_{3}\right\rangle=-\sin \theta|\alpha \beta\rangle+\cos \theta|\beta \alpha\rangle \\
& \frac{E_{1}=-\bar{\omega}+\frac{D}{4}}{\left|\phi_{1}\right\rangle=|\alpha \alpha\rangle}
\end{aligned}
$$

Figure 1.3: Eigenstates and eigenvalues of the Hamiltonian in Equation 1.48 for two dipole coupled spins $\frac{1}{2}$ with different chemical shifts. The symbol $\bar{\omega}$ is the average chemical shift $\frac{\langle\Delta \omega\rangle_{2}+\langle\Delta \omega\rangle_{2}}{2}$, the symbol $\delta \omega$ is the chemical shift difference between the coupled nuclei, and $\theta$ is defined by $\tan 2 \theta=\frac{-D}{2 \delta \omega}$.

NMR spectra and the correlation of peaks in the two dimensions to map out either through-bond or through-space connectivities between atoms in a molecule. All two-dimensional NMR experiments can be separated into four parts : preparation, evolution $\left(t_{1}\right)$, mixing, and detection $\left(t_{2}\right)$. The preparation and mixing periods are usually composed of one or more RF pulses, while the evolution and detection periods are time periods during which evolution under the Hamiltonian occurs. A two-dimensional experiment is usually performed as a series of one-dimensional experiments with the value of $t_{1}$ incremented between each experiment. The resulting two-dimensional time-domain data matrix is Fourier Transformed along two dimensions to give the desired frequency-domain spectrum.

The class of two-dimensional experiment of interest in this thesis is COSY (COrrelated SpectroscopY). The simplest COSY experiment is the one first proposed by 
Jeene. [14]. It has preparation and mixing periods which each consist of a single $\frac{\pi}{2}$ pulse, and free evolution during ihe evolution and detection periods. The pulse sequence can be written :

$$
\left(\frac{\pi}{2}\right)_{y}-t_{1}-\left(\frac{\pi}{2}\right)_{y}-\dot{t_{2}}
$$

The observed signal is :

$$
S\left(t_{1}, t_{2}\right)=\operatorname{Tr}\left(e^{-i \mathcal{H} t_{2}} e^{-i \frac{\pi}{2} I_{y}} e^{-i \mathcal{H} t_{1}} e^{-i \frac{\pi}{2} I_{y}} \rho_{\mathrm{eq}} e^{i \frac{\pi}{2} I_{\nu}} e^{i \mathcal{H} t_{1}} e^{i \frac{\pi}{2} I_{\nu}} e^{i \mathcal{H} t_{2}} I^{+}\right) .
$$

Kearranging the trace, expanding in the eigenstates of the Hamiltonian, and evaluating the result of the first pulse, as in Equation 1.54, this becomes :

$$
S\left(t_{1}, t_{2}\right)=\sum_{i, j, k, l}\left\langle i\left|e^{-i \frac{\pi}{2} I_{\nu}} e^{-i \mathcal{H} t_{1}}\right| k\right\rangle\left\langle k\left|I_{x} e^{i \mathcal{H} t_{1}}\right| l\right\rangle\left\langle l\left|e^{i \frac{\pi}{2} I_{\nu}} e^{i \mathcal{H} t_{2}}\right| j\right\rangle\left\langle j\left|I^{+} e^{-i \mathcal{H} t_{2}}\right| i\right\rangle
$$

which can be written :

$$
S\left(t_{1}, t_{2}\right)=\sum_{i, j, k, l} e^{i\left(\omega_{l}-\omega_{k}\right) t_{1}} e^{i\left(\omega_{j}-\omega_{i}\right) t_{2}}\left\langle i\left|e^{-i \frac{\pi}{2} I_{y}}\right| k\right\rangle\left\langle k\left|I_{x}\right| l\right\rangle\left\langle l\left|e^{i \frac{\pi}{2} I_{\nu}}\right| j\right\rangle\left\langle j\left|I^{+}\right| i\right\rangle
$$

or

$$
S\left(t_{1}, t_{2}\right)=\sum_{i, j, k, l} e^{i\left(\omega_{l}-\omega_{k}\right) t_{1}} e^{i\left(\omega_{j}-\omega_{i}\right) t_{2}} Z_{j i, k l}
$$

where

$$
Z_{j i, k l}=\left\langle i\left|e^{-i \frac{\pi}{2} I_{y}}\right| k_{i}\right\rangle\left\langle k\left|I_{x}\right| l\right\rangle\left\langle l\left|e^{i \frac{\pi}{2} I_{y}}\right| j\right\rangle\left\langle j\left|I^{+}\right| i\right\rangle .
$$

This results in sixteen peaks at frequencies in the two dimensions corresponding to all possible pairs of the frequencies $\omega_{21}, \omega_{31}, \omega_{42}$, and $\omega_{43}$. Since all peaks in a twodimensional spectrum of a strongly coupled spin system can not be simultaneously phased, all two-dimensional spectra in this thesis will be presented in absolute value mode. The absolute value intensities of the peaks are given by $\left[\left|Z_{j i, k l}\right|^{2}+\left|Z_{j i, l k}\right|^{2}\right]^{\frac{1}{2}}$ and are listed in Figure 1.4. The peaks with frequencies that are the same in the 
two dimensions are called diagonal peaks. The peaks with frequencies which are different in the two dimensions are called cross-peaks and only appear if two nuclei are coupled in some fashion. It is the presence or absence of these cross peaks that allows information about connectivities in a molecule to be determined, as will be discussed in Chapter 3.

The basic COSY experiment in Equation 1.64 can become a double-quantum filtered COSY with the addition of a delay and another $\frac{\pi}{2}$ pulse to the mixing period :

$$
\left(\frac{\pi}{2}\right)_{\phi}-t_{1}-\left(\frac{\pi}{2}\right)_{\phi}-\tau_{1}-\left(\frac{\pi}{2}\right)_{y}-t_{2}
$$

which can be written in the density matrix formalism (assuming $\phi=y$ ) :

$$
S\left(t_{1}, t_{2}\right)=\sum_{i, j, k, l, m, n} e^{i\left(\omega_{l}-\omega_{k}\right) t_{1}} e^{i\left(\omega_{j}-\omega_{i}\right) t_{2}} e^{i\left(\omega_{n}-\omega_{m}\right) \tau_{1}} Z_{j i, k l, m n}
$$

where

$$
Z_{j i, k l, m n}=\left\langle i\left|e^{-i \frac{\pi}{2} I_{y}}\right| m\right\rangle\left\langle m\left|e^{-i \frac{\pi}{2} I_{y}}\right| k\right\rangle\left\langle k\left|I_{x}\right| l\right\rangle\left\langle l\left|e^{i \frac{\pi}{2} I_{y}}\right| n\right\rangle\left\langle n\left|e^{i \frac{\pi}{2} I_{y}}\right| j\right\rangle\left\langle j\left|I^{+}\right| i\right\rangle
$$

The first two pulses in this sequence are cycled through phases $\phi=0^{\circ}, 90^{\circ}, 180^{\circ}$, and $270^{\circ}$ and the receiver phase must be cycled through $0^{\circ}$ and $180^{\circ}$ to select only the double quantum transitions during $\tau_{1}$. This means that $m$ and $n$ in the above equation take only the values 1 and 4 . The frequencies of the peaks from this experiment are the same as in the simple COSY experiment, but the intensities of the peaks are different and are given in Figure 1.4.

For the experiments in Chapter 3, it will be useful to do this experiment with a $(\pi)_{\phi}$ pulse during $t_{1}$ to refocus chemical shift during that time pericd. In the density matrix formalism, this becomes :

$$
S\left(t_{1}, t_{2}\right)=\sum_{i, j, k, l, m, n, p, q} e^{i\left(\omega_{l}-\omega_{k}\right) \frac{t_{1}}{2}} e^{i\left(\omega_{q}-\omega_{p}\right) \frac{t_{1}}{2}} e^{i\left(\omega_{j}-\omega_{i}\right) t_{2}} e^{i\left(\omega_{n}-\omega_{m}\right) \tau_{1}} Z_{j i, k l, m n, p q}
$$




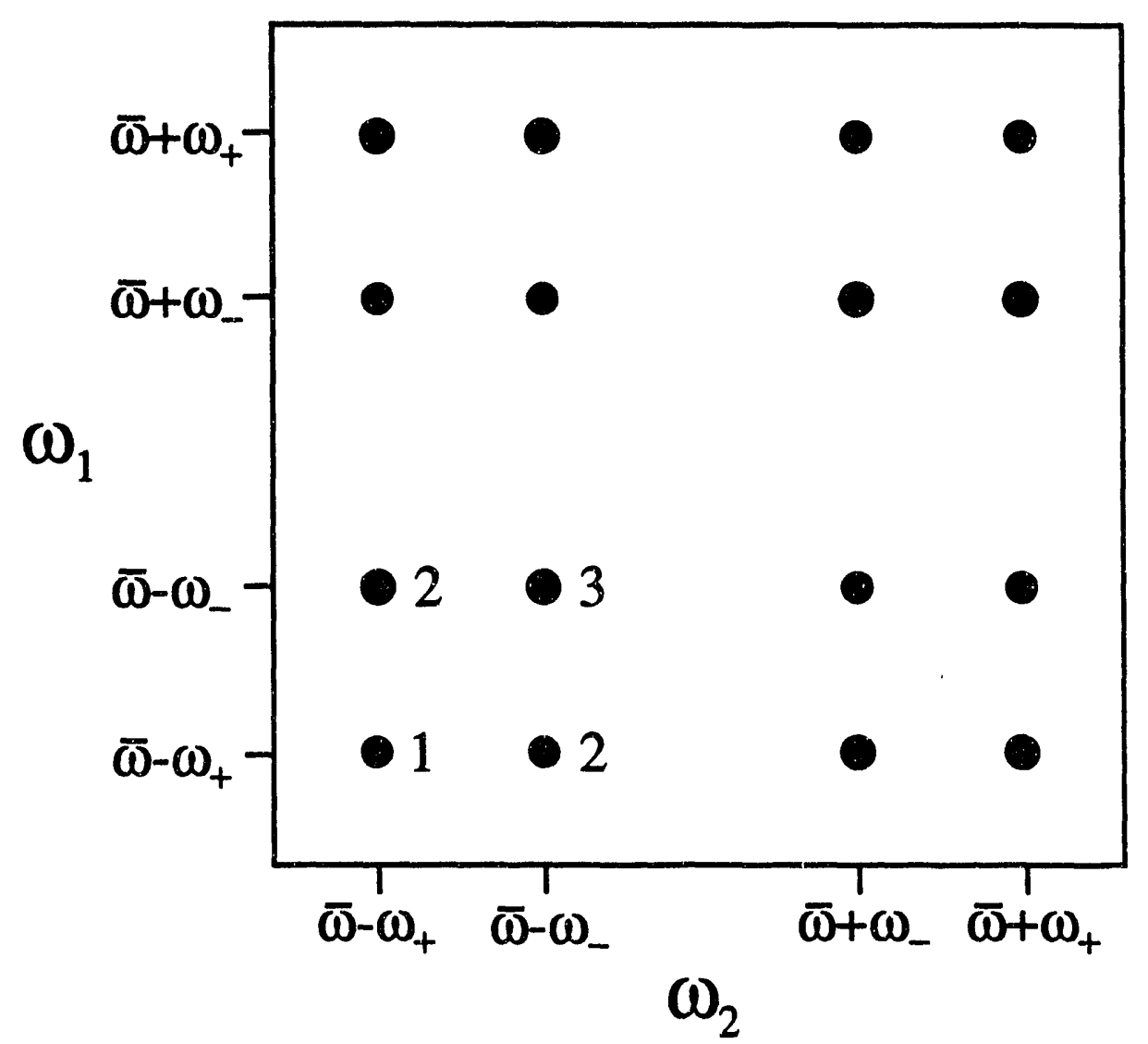

Figure 1.4: The pattern of peaks which arises in a simple COSY experiment on a dipole-coupled two-proton system. $\bar{\omega}$ is the average of the chemical shifts of the two coupled nuclei and $\omega_{ \pm}=\frac{\mathrm{D}}{2} \pm \frac{1}{2} \sqrt{(\delta \omega)^{2}+\frac{\bar{D}^{2}}{4}}$. The absolute-valued intensity of the peaks are : 1 . $(1-\sin 2 \theta) \sqrt{1+\sin ^{2} 2 \theta}, 2 \cdot \cos ^{2} 2 \theta$, and 3 . (1+ $\sin 2 \theta) \sqrt{1+\sin ^{2} 2 \theta}$ [15]. The intensities of the peaks that are not numbered can be determined from the $D_{4 h}$ symmetry of the absolute-valued spectrum. For a double-quantum-filtered COSY experiment, the absolute-valued intensities of the numbered peaks are : $1 .(1-\sin 2 \theta)^{2}, 2 \cdot \cos ^{2} 2 \theta$, and $3 .(1+\sin 2 \theta)^{2}$. 
where

$$
\begin{aligned}
Z_{j i, k l, m n, p q}= & \left\langle i\left|e^{-i \frac{\pi}{2} I_{\nu}}\right| m\right\rangle\left\langle m\left|e^{-i \frac{\pi}{2} I_{y}}\right| p\right\rangle\left\langle p\left|e^{-i \pi I_{y}}\right| k\right\rangle\left\langle k\left|I_{x}\right| l\right\rangle \\
& \left\langle l\left|e^{i \pi I_{y}}\right| q\right\rangle\left\langle q\left|e^{i \frac{\pi}{2} I_{\nu}}\right| n\right\rangle\left\langle n\left|e^{i \frac{\pi}{2} I_{y}}\right| j\right\rangle\left\langle j\left|I^{+}\right| i\right\rangle
\end{aligned}
$$

The peak positions and intensities for this experiment are given in Figure 1.5. This is the experiment that will be used (with small modifications) in Chapter 3 to study the conformation and orientation of $n$-alkanes dissolved in liquid crystals. 


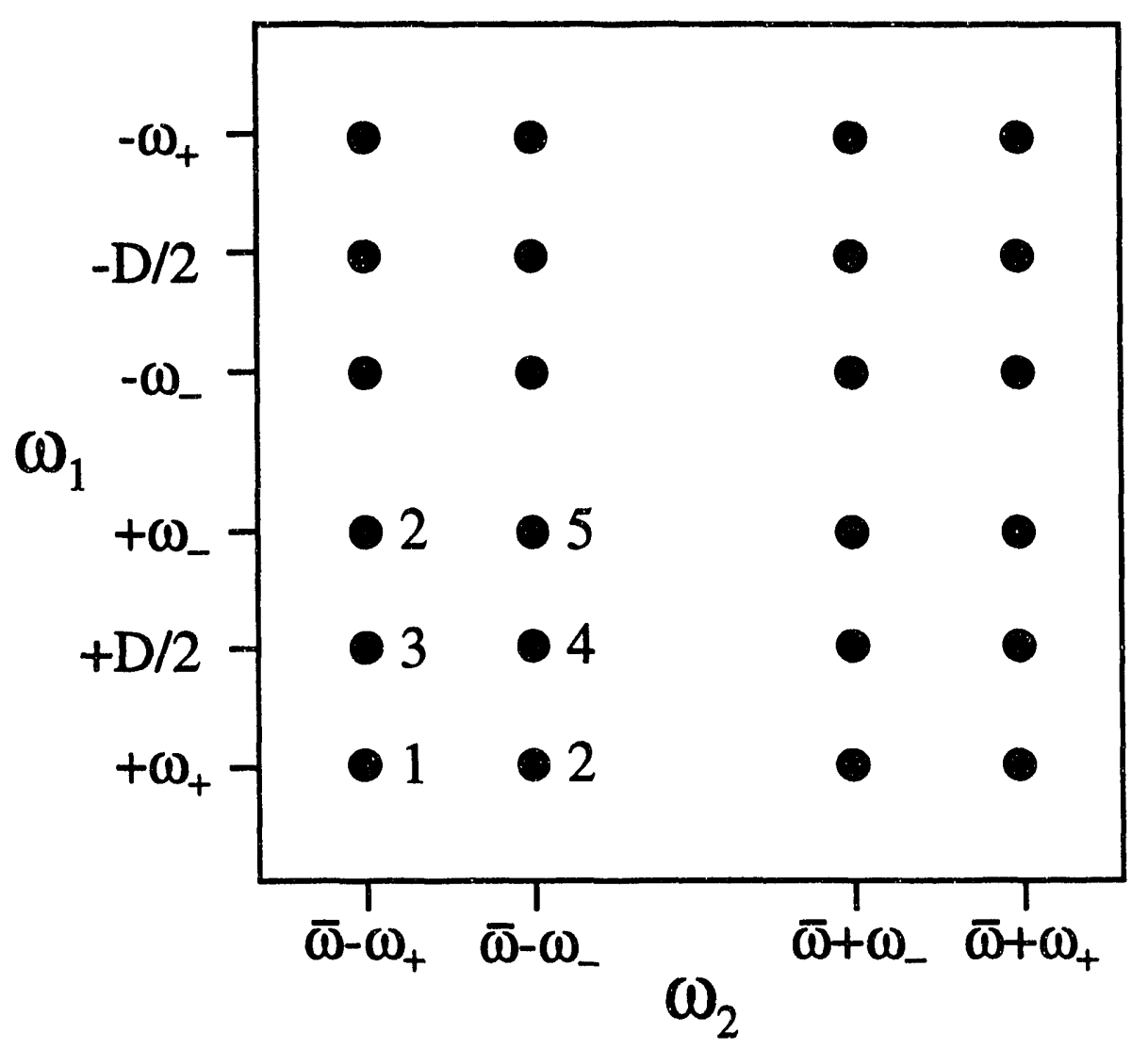

Figure 1.5: The pattern of peaks which arises in a refocussed doublequantum-filtered COSY spectrum of a dipole-coupled two-proton system. $\bar{\omega}$ is the average of the chemical shifts of the two coupled nuclei and $\omega_{ \pm}=$ $\frac{\mathrm{D}}{2} \pm \frac{1}{2} \sqrt{(\delta \omega)^{2}+\frac{\mathrm{D}^{2}}{4}}$. The intensities of the numbered peaks are : 1 . $(1-$ $\sin 2 \theta)^{2} \sin 2 \theta, 2 . \cos ^{2} 2 \theta \sin 2 \theta, 3.2 \cos ^{2} 2 \theta(1-\sin 2 \theta), 4.2 \cos ^{2} 2 \theta(1+\sin 2 \theta)$, and $5 .(1+\sin 2 \theta)^{2} \sin 2 \theta$. 


\section{Chapter 2}

\section{Liquid Crystals}

\subsection{Introduction}

Liquid crystals comprise what is essentially a fourth state of matter. They have been the subject of intense study over the last 30 years, not only for their interesting phase behavior, but also for their applications in displays and other optical technology. Numerous books and reviews $[16,17,18,19,20,21,22,23,24]$ exist which can provide a more thorough description of their properties and applications than is presented here, but an attempt will be made to provide some background which is relevant to the upcoming work in this thesis.

As the name implies, liquid crystals have properties of both liquids and solids and, because of this intermediate phase behavior, are also called mesophases. They are similar to liquids in that molecules in both phases undergo rapid translational and rotational motion. A typical self-diffusion constant for a nematic liquid crystal is $10^{-6}$ to $10^{-7} \mathrm{~cm}^{2} \mathrm{~s}^{-1}$ [16] compared with the self-diffusion constant of small solutes in water of $10^{-5} \mathrm{~cm}^{2} \mathrm{~s}^{-1}[25]$. The unique properties of liquid crystals, how- 

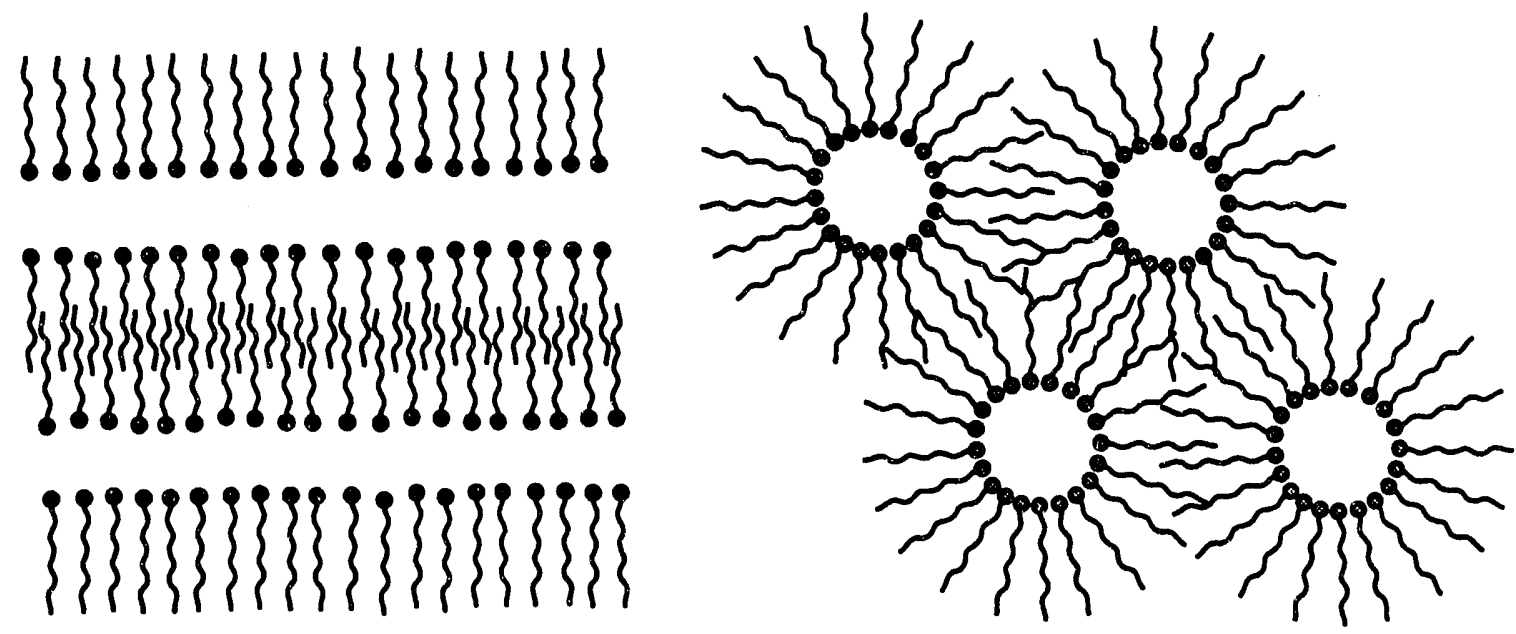

Figure 2.1: A schematic picture of laminar (left) and hexagonal (right) phases of lyotropic liquid crystals. The molecules' polar head groups are oriented toward the solvent water, while the hydrophobic hydrocarbon tails exclude the water. The molecules in the laminar liquid crystal are arranged in sheets separated by layers of water, and the hexagonal phase has the water confined to tubes which are arranged in a hexagonal lattice.

ever, come from their solid-like attributes. Molecules in liquid crystals, like in solids, have constraints on their orientational and, in some cases, translational degrees of freedom. These constraints break the isotropic symmetry of the liquid state. The existence of a director, $\mathbf{n}$, usually defined as the preferred direction of alignment of the liquid crystal molecules, is one consequence of the broken symmetry.

Liquid crystals can be separated into two broad classes based on the origin of their liquid crystalline properties. The first class is lyotropic, which includes those mesophases formed from mixtures containing compounds with amphiphilic properties and solvents. Their liquid crystalline behavior is determined by the concentrations of the components of the mixture. Some common examples of such systems are soaps or detergents in water and phospholipids in water. They form laminar, micellar, and other structures, as shown in Figure 2.1. 
The other class of liquid crystals is known as thermotropic. These liquid crystals are usually formed from a single compound which does not directly undergo a phase transition from the solid to liquid phase, but rather exhibits one or more liquid crystalline phases in the temperature range between its solid and liquid phases. Thermotropic liquid crystals can be formed from rod-shaped organic molecules, disc-shaped molecules [23], polymers [17], and organometallic compounds $[26,27]$, but the most common ones (and the ones of primary interest here) are the rod-shaped organic molecules. These liquid crystals can form several types of mesophases, differing in the nature of the constraints placed on the orientation and position of the liquid crystal molecules. The three most common types are nematic, chiral nematic (also known as cholesteric), and smectic.

\subsection{Nematic Liquid Crystals}

Nematic liquid crystals are the most liquid-like of the three types. The liquid crystal molecules have constraints on their orientation, but there is no positional order of the molecules' centers of mass. Schematic pictures of nematic mesophases composed of rod- and disc-shaped molecules are shown in Figure 2.2.

The long axes of the rod-shaped molecules tend to align in the same direction as their neighbors, leading, on average, to a preferred direction of alignment of the molecules called the director, n. For disc-shaped molecules, this is the preferred direction of alignment of the normals to the discs. A single director is all that is needed to characterize the anisotropy in most nematics, since they are uniaxial (though a few biaxial nematics have been shown to exist [28]). In all known nematics the directions $+\mathbf{n}$ and $-\mathbf{n}$ are indistinguishable. 


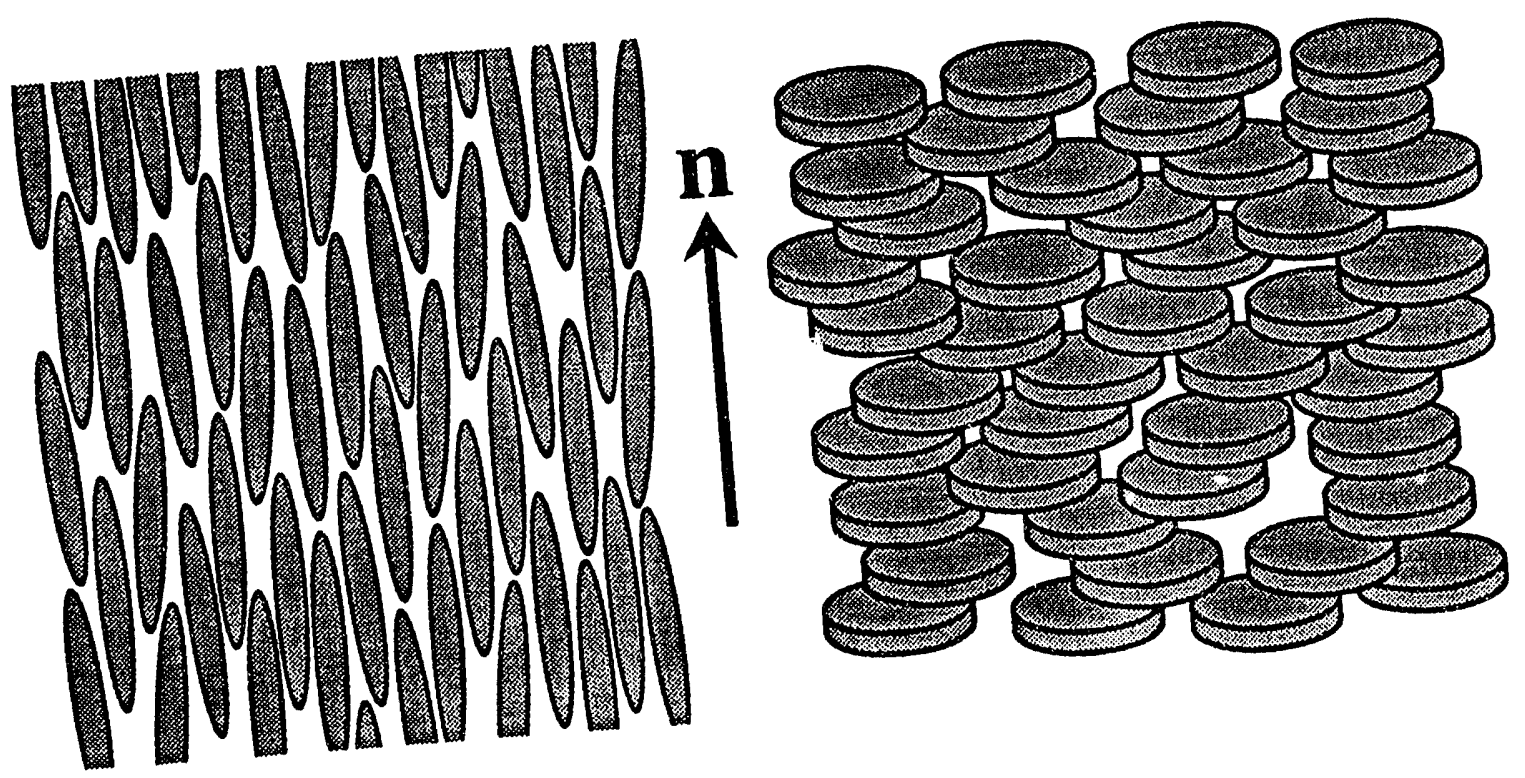

Figure 2.2: Schematic pictures of nematic liquid crystals composed of rod- and disc-shaped molecules. The liquid crystal molecules have constraints on their orientation but not on their position.

The direction of $\mathbf{n}$ in a given liquid crystal sample is arbitrary and may vary throughout the sample, but the variations are on a length scale much larger than the molecular length [18]. The direction of $\mathbf{n}$ is usually imposed by the boundaries of the sample or by applied electric or magnetic fields. Magnetic fields can impose alignment of the liquid crystal molecules with their long axes either parallel or perpendicular to the field, depending on the diamagnetic anisotropy, $\Delta \chi\left(=\chi_{\|}-\right.$ $\chi_{\perp}$ ), of the liquid crystal molecules. If $\Delta \chi$ is greater than zero, the liquid crystal molecules align with their long axes parallel to the applied magnetic field, and if $\Delta \chi$ is less than zero, the molecules align perpendicular to the applied field. In large magnetic fields like the 8.4 T field used in the studies described in the next chapter, the liquid crystal molecules are completely aligned by the field, with only transient variations of $\mathbf{n}$ within the sample.

One example of a thermotropic nematic liquid crystal is shown in Figure 2.3. 


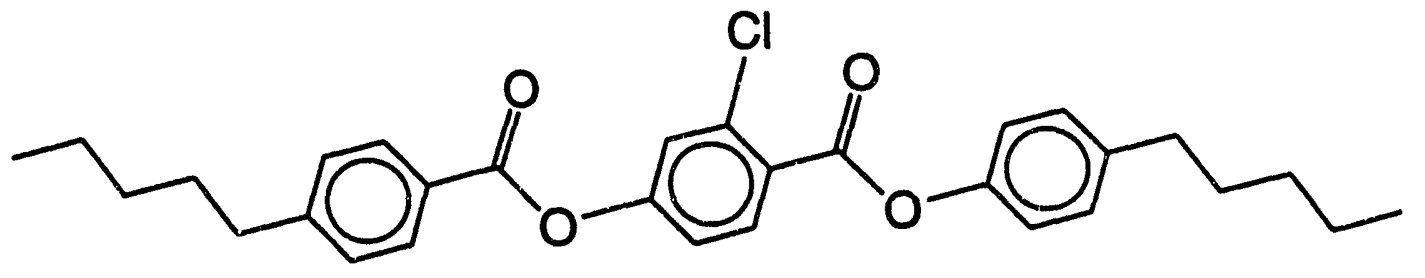

Figure 2.3: The nematic liquid crystal used for the studies in this thesis. It will be referred to in the rest of this thesis by its Eastman Kodak catalog number, EK11650. It melts at $39^{\circ} \mathrm{C}$ to form a nematic which turns into an isotropic liquid at $122^{\circ} \mathrm{C}$.

This liquid crystal is the one that will be used in the studies in the next chapter. It has a large nematic range, from $39^{\circ} \mathrm{C}$ to $122^{\circ} \mathrm{C}$, and has a positive diamagnetic anisotropy so that the long axes of the molecules align parallel to an applied magnetic field. The studies in the next chapter involve molecules dissolved in liquid crystals. The addition of solute molecules to liquid crystals, in general, has the effect of lowering the the nematic-isotropic transition temperature and narrowing the nematic temperature range of the liquid crystal.

\subsection{Chiral Nematic (Cholesteric) Liquid Crys- tals}

If a nematic liquid crystal is made up of one enantiomer of a chiral molecule, it is called a chiral nematic or cholesteric liquid crystal. This type of liquid crystal is very similar to a nematic except that the direction of the director in the liquid crystal varies throughout the sample in a regular fashion. The direction of the director varies along an axis in a helical twist with a pitch on the order of $2000 \AA$ or more $[16,18]$. Figure 2.4 shows a schematic picture of such a liquid crystal. The 


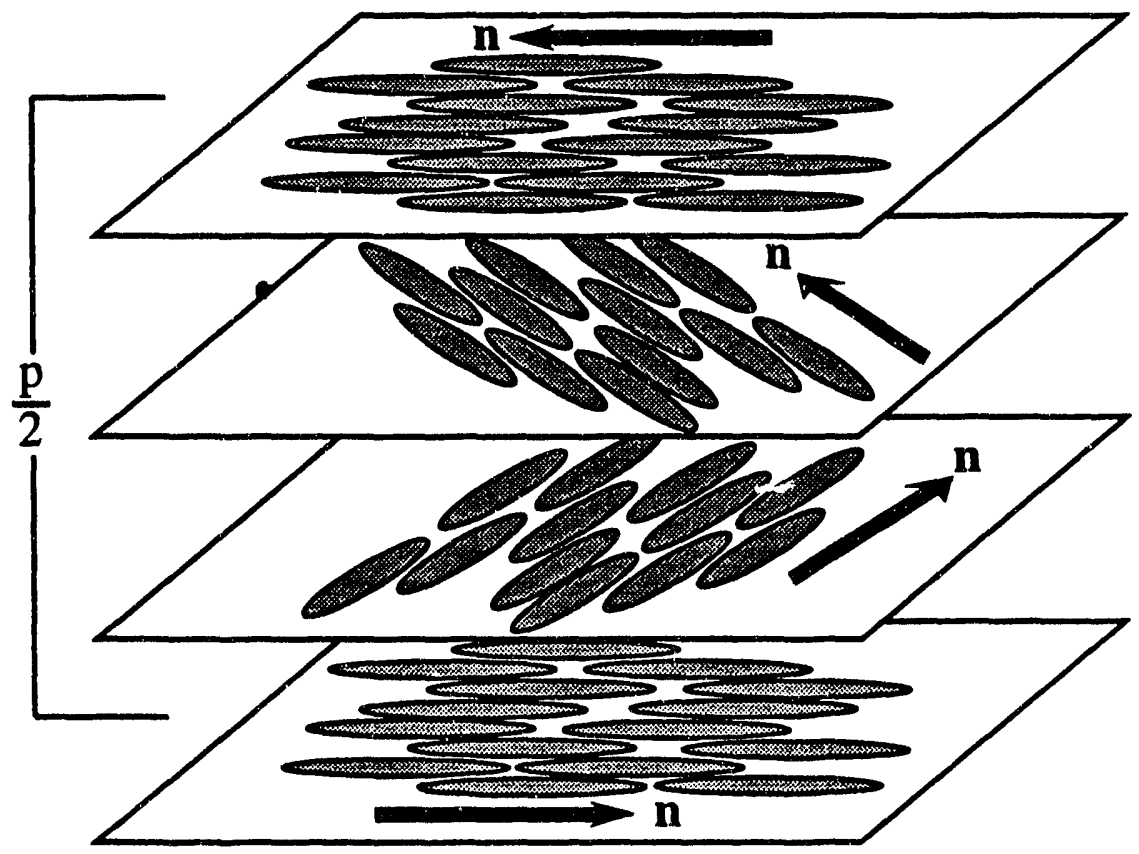

Figure 2.4: A schematic picture of a Chiral Nematic (or Cholesteric) liquid crystal composed of rod-shaped molecules. The director varies along an axis in a helix with pitch $\mathrm{p}$.

pitch of the twist can be modified by the application of weak electric or magnetic fields, or removed completely by the application of stronger fields.

\subsection{Smectic Liquid Crystals}

Smectic liquid crystals are more ordered than nematic or cholesteric liquid crystals. They have constraints on molecular postion as well as orientation. There are many different types of smectic liquid crystals, two of which are shown in Figure 2.5. The Smectic A liquid crystal is essentially a nematic in which the positions of the molecules have been restricted to layers perpendicular to the director. Translation in the two dimensions within a layer is not much different from a nematic, but 


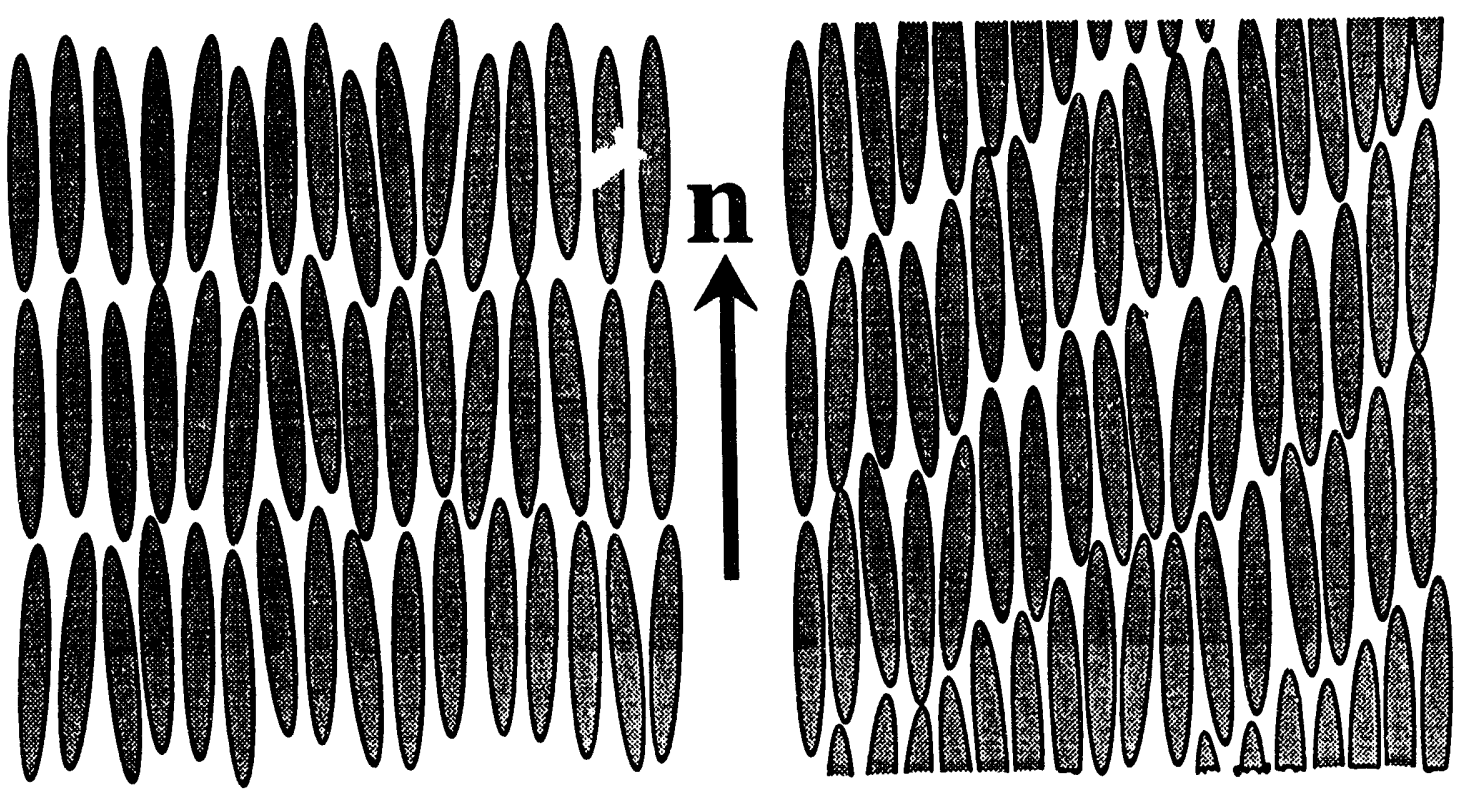

Figure 2.5: Schematic pictures of Smectic A (left) and Smectic C (right) liquid crystals composed of rod-shaped molecules. In addition to constraints on molecular orientation, molecular position is constrained in one dimension.

perpendicular to the layers, it is significantly restricted. Smectic C liquid crystals are similar to Smectic A liquid crystals except that the layers of positional localization are not perpendicular to the director, but at some angle to it. An example of a liquid crystal that has a Smectic A phase is $4^{\prime}$-n-octyl-4-cyanobiphenyl (8-CB) which melts to form a Smectic A phase at $21.5^{\circ} \mathrm{C}$. It has a transition to a nematic liquid crystal at $33.5^{\circ} \mathrm{C}$ and then a transition to an isotropic liquid at $40.5^{\circ} \mathrm{C}$.

\subsection{Other Mesophases}

Besides these three common types of thermotropic liquid crystal phases, two others have been of much interest recently - columnar phases and blue phases. Columnar liquid crystals are formed from disc-shaped molecules and have not only orienta- 

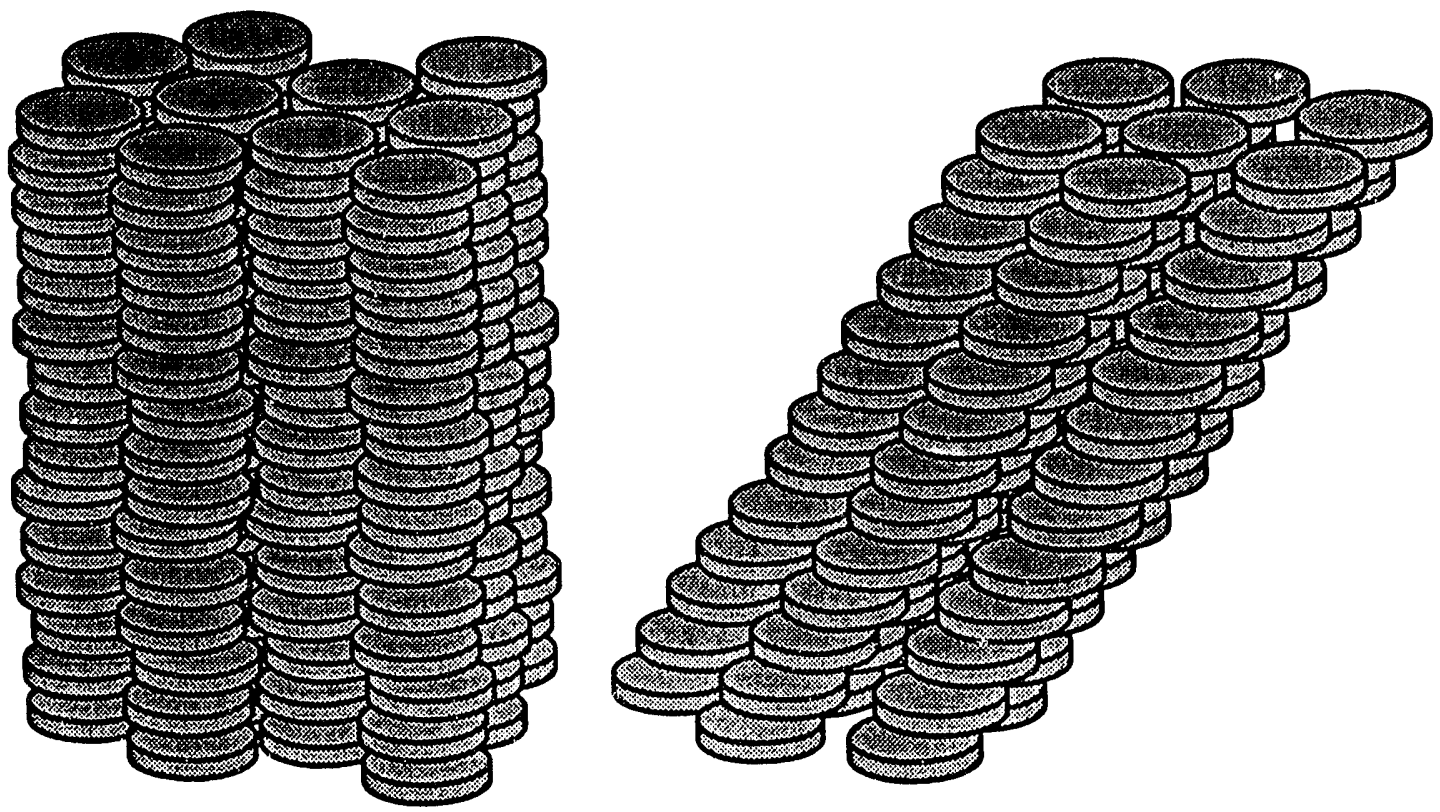

Figure 2.6: Schematic pictures of orthogonal (left) and tilted (right) columnar liquid crystals. Molecular orientation and molecular position in two dimensions are constrained, forming columns of molecules which are arranged in a "lattice".

tional order, but also constraints on molecular translation in two dimensions, with translation possible in the third [23]. Two examples of columnar mesophases are shown in Figure 2.6. The discotic molecules are oriented as in a discotic nematic, and their positions are constrained in two dimensions into columns which are arranged in a "lattice". Molecular translation is less restricted along the column axis. Some examples of the types of molecules which form discotic nematic and columnar liquid crystalline phases are shown in Figure 2.7.

Blue phases are formed by sorne chiral nematic liquid crystals with short pitches $(<3000) \AA[17]$ in a narrow temperature range between the chiral nematic and isotropic phases. Three blue phases are known - BPI, BPII, and BPIII - and typically form in that order with increasing temperature. The phases are found to 

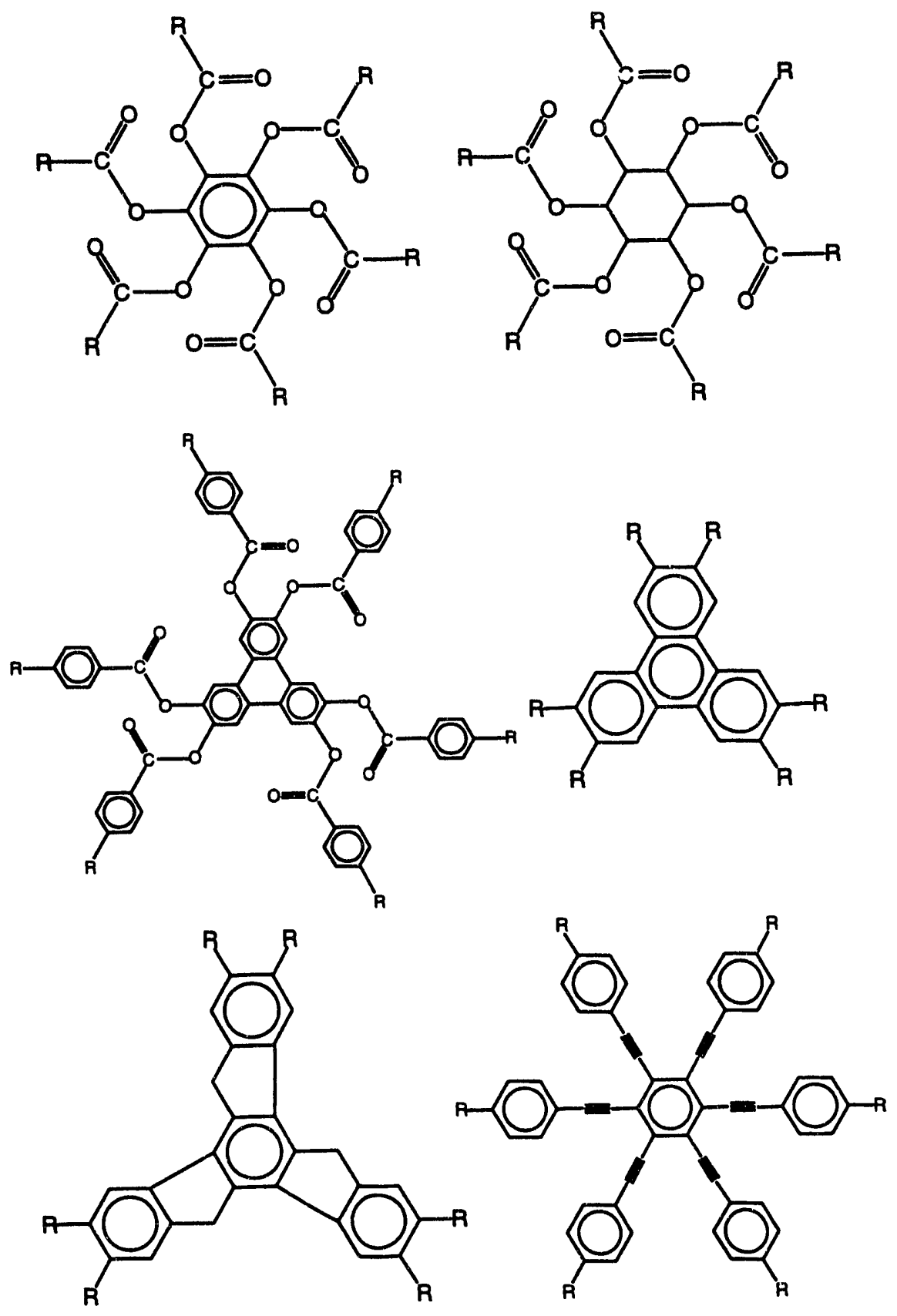

Figure 2.7: Some types of discotic mesogenic cores. The "R" substituents are usually alkyl or alkoxy chains, but may also have more complicated structures. The nature of these substituents has a dramatic effect on the phase behavior of the liquid crystals [23]. 
be optically isotropic (unlike other liquid crystal phases) and have quite different symmetries than other liquid crystals. BPI has body centered cubic symmetry, BPII has simple cubic symmetry, and BPIII has either icosahedral symmetry or is amorphous [17, 24]. One example of a molecule which exhibits all three Blue Phases is cholesterol nonanoate.

\subsection{Order Parameters}

The mesophases described in the previous sections all have a director which can be defined as a direction of preferred alignment of the molecules. The degree to which the r.olecules are aligned along the director is measured by the order parameter of the liquid crystal. The order parameter is a macroscopic measure of order, that is, it is an ensemble averaged quantity, averaged over all molecules in the sample. The order parameter should vary from zero (in an isotropic liquid) to a maximum (in a completely ordered crystal).

Consider a single molecule of a rod-shaped nematic liquid crystal. The orientation of the long axis of the molecule with respect to a laboratory-fixed axis system (with the director of the liquid crystal along the z-axis) can be described by a vector a. A first-rank tensor like this vector cannot provide an order parameter for the liquid crystal, berause an ensemble average of the direction of a vector in a nematic liquid crystal will always be zero due to the equivalence of $+\mathbf{n}$ and $-\mathbf{n}$. A second-rank tensor constructed from this vector, however, does provide an order parameter. The Cartesian order tensor, $S_{\alpha \beta}$, can be constructed from the Cartesian components, $a_{\alpha}$, of a :

$$
S_{\alpha \beta}=\left\langle\frac{1}{2}\left(3 a_{\alpha} a_{\beta}-\delta_{\alpha \beta}\right)\right\rangle
$$


or since the components of a are direction cosines for the vector in the lab-fixed axis system :

$$
S_{\alpha \beta}=\left\langle\frac{1}{2}\left(3 \cos \theta_{\alpha} \cos \theta_{\beta}-1\right)\right\rangle
$$

where $\theta_{\chi}(\chi=x, y, z)$ is the angle between the vector a and the $\chi$-axis of the laboratory-fixed axis system. This can be rewritten in terms of Euler angles $\alpha$ and $\beta$ describing ine orientation of a with respect to the laboratory-fixed axis system :

$$
\begin{aligned}
S_{z z} & =\left\langle\frac{1}{2}\left(3 \cos ^{2} \beta-1\right)\right\rangle \\
S_{x x-y y} & =\left\langle\frac{3}{2} \sin ^{2} \beta \cos 2 \alpha\right\rangle \\
S_{x y} & =\left\langle\frac{3}{2} \sin ^{2} \beta \sin \alpha \cos \alpha\right\rangle \\
S_{x z} & =\left\langle\frac{3}{2} \sin \beta \cos \beta \cos \alpha\right\rangle \\
S_{y z} & =\left\langle\frac{3}{2} \sin \beta \cos \beta \sin \alpha\right\rangle .
\end{aligned}
$$

If the molecule is cylindrically symmetric, then only the component of $S_{\alpha \beta}$ along the director of the liquid crystal is non-zero :

$$
S_{z z}=\frac{1}{2}\left\langle 3 \cos ^{2} \beta-1\right\rangle
$$

Rod-shaped nematic liquid crystals usually deviate only slightly from cylindrical symmetry, so this is the most important component of the order tensor. It is a measure of the average orientation of the long axis of the molecule and ranges from a value of 1 for a perfectly ordered crystal, to zero for an isotropic liquid, to $-\frac{1}{2}$ for a perfectly ordered crystal with the long axes of the molecules aligned perpendicular to the director. Typical nematic order parameters range from 0.3 to 0.8 [18]. 


\subsection{NMR in Liquid Crystals}

NMR has proven to be a very useful tool for studying liquid crystals. NMR studies of liquid crystals can provide information about molecular ordering, phase structure, molecular structure, molecular conformation, and molecular dynamics $[13,20,29$, 30]. This thesis is primarily concerned with the use of NMR to study molecular ordering and conformation in liquid crystals, so this will be discussed in detail.

As stated in the previous section, the order parameter used to describe liquid crystals is a second-rank tensor quantity. To measure this using NMR, one must measure a second-rank property of the nuclear spin system like chemical shift anisotropy, quadrupole coupling, or dipole coupling $[13,30]$. Starting with a secondrank Hamiltonian in its principle axis system (PAS) :

$$
\mathcal{H}=\sum_{q=-2}^{2}(-1)^{-q} A_{2 q}^{\mathrm{PAS}} T_{2-q}
$$

containing spatial $A_{2 q}^{\text {PAS }}$ and spin $T_{2-q}$ terms, the spatial part can be expressed in a molecule-fixed frame by a rotation of coordinates through an angle $\Omega_{1}$ :

$$
A_{2 q}^{\mathrm{MOL}}=\sum_{r=-2}^{2} A_{2 r}^{\mathrm{PAS}} D_{r q}^{2}\left(\Omega_{1}\right)
$$

The resulting tensor can be expressed in the director frame by a rotation through an angle $\Omega_{2}$ :

$$
A_{2 q}^{\mathrm{DIR}}=\sum_{s=-2}^{2} A_{2 s}^{\mathrm{MOL}} D_{s q}^{2}\left(\Omega_{2}\right) .
$$

If the director is parallel to the applied magnetic field, as it will be for liquid crystals with a positive diamagnetic anisotropy $(\Delta \chi>0)$, then no further transformations are necessary since $A_{2 q}^{\mathrm{LAB}}=A_{2 q}^{\mathrm{DIR}}$. If the liquid crystalline phase is uniaxial, all 
terms with $q \neq 0$ vanish because of the cylindrical symmetry of a uniaxial phase :

$$
A_{20}^{\mathrm{LAB}}=\sum_{s=-2}^{2} A_{2 s}^{\mathrm{MOL}} D_{s 0}^{2}\left(\Omega_{2}\right) .
$$

This gives the spatial part of the Hamiltonian for a particular molecule at a particular orientation at a given instant. Since in most NMR spectra of liquid crystals, narrow, motionally-averaged lines are observed, it is safe to assume that the molecular motions are occurring much faster than the time-scale of the NMR experiment; the observed spectra are averaged over all molecular motions. This means that we must take a long-time average of the spatial tensor in Equation 2.8 :

$$
A_{20}^{\mathrm{DIR}}=\sum_{s=-2}^{2} A_{2 s}^{\mathrm{MOL}}\left\langle D_{s 0}^{2}\left(\Omega_{2}\right)\right\rangle .
$$

The assumption has been made that the angle $\Omega_{1}$ between the PAS of the NMR interaction and the molecule-fixed axis system remains fixed; otherwise, an average over this angle would also have to be performed. The $\left\langle D_{s 0}^{2}\left(\Omega_{2}\right)\right\rangle$ are order parameters describing the average orientation of the molecule-fixed axis system with respect to the laboratory (director) frame z-axis. The entire Hamiltonian then becomes :

$$
\begin{aligned}
\mathcal{H} & =A_{20}^{\mathrm{LAB}} T_{20} \\
\mathcal{H} & =\sum_{s=-2}^{2} A_{2 s}^{\mathrm{MOL}}\left\langle D_{s 0}^{2}\left(\Omega_{2}\right)\right\rangle T_{20} .
\end{aligned}
$$

If the liquid crystal has $\Delta \chi<0$, that is, it orients with the director perpendicular to the applied magnetic field, then a rotation from the director frame to the lab frame is required. Equation 2.11 then becomes :

$$
\mathcal{H}=\sum_{s=-2}^{2} A_{2 s}^{\mathrm{MOL}}\left\langle D_{s t}^{2}\left(\Omega_{2}\right)\right\rangle D_{t, 0}^{2}\left(\Omega_{3}\right) T_{20}
$$

where $\Omega_{3}$ is the angle between the director and the applied magnetic field. Since the liquid crystal is uniaxial, however, the order parameters $\left\langle D_{s t}^{2}\left(\Omega_{2}\right)\right\rangle$ cannot depend 
on the third Euler angle $\gamma$ so they become $\left\langle D_{s 0}^{2}\left(\Omega_{2}\right)\right\rangle$ and Equation 2.12 becomes :

$$
\mathcal{H}=\sum_{s=-2}^{2} A_{2 s}^{\mathrm{MOL}}\left\langle D_{s 0}^{2}\left(\Omega_{2}\right)\right\rangle D_{0,0}^{2}\left(\Omega_{3}\right) T_{20}
$$

For liquid crystals with the director perpendicular to the applied magnetic field, the angle $\Omega_{3}=\left(\alpha=0, \beta=\frac{\pi}{2}, \gamma=0\right)$ so $D_{0,0}^{2}\left(\Omega_{3}\right)=\frac{1}{2}\left(3 \cos ^{2} \beta-1\right)=-\frac{1}{2}$, and Equation 2.13 becomes :

$$
\mathcal{H}=-\frac{1}{2} \sum_{s=-2}^{2} A_{2 s}^{\mathrm{MOL}}\left\langle D_{s 0}^{2}\left(\Omega_{2}\right)\right\rangle T_{20}
$$

Thus, the only difference between the cases of uniaxial liquid crystals with positive and negative diamagnetic anisotropies is that with a negative diamagnetic anisotropy the dipole couplings are scaled by a factor of $-\frac{1}{2}$.

Continuing with the case of liquid crystals with positive diamagnetic anisotropy from Equation 2.11, it is usually easier to work in Cartesian coordinates. The spherical tensor $A_{2 s}^{\mathrm{MOL}}$ can be rewritten in Cartesian tensor form, and the Wigner rotation matrices can be written in terms of Euler angles :

$$
\begin{aligned}
\mathcal{H}= & \left\{\frac{1}{\sqrt{6}}\left[2 A_{z z}^{\mathrm{MOL}}-\left(A_{x x}^{\mathrm{MOL}}+A_{y y}^{\mathrm{MOL}}\right)\right]\left\langle\frac{1}{2}\left(3 \cos ^{2} \beta-1\right)\right\rangle\right. \\
& +\frac{1}{2}\left[A_{x z}^{\mathrm{MOL}}+A_{z x}^{\mathrm{MOL}}+i\left(A_{y z}^{\mathrm{MOL}}+A_{z y}^{\mathrm{MOL}}\right)\right]\left\langle\sqrt{\frac{3}{2}} \sin \beta \cos \beta e^{-i \alpha}\right\rangle \\
& +\frac{1}{2}\left[A_{x z}^{\mathrm{MOL}}+A_{z x}^{\mathrm{MOL}}-i\left(A_{y z}^{\mathrm{MOL}}+A_{z y}^{\mathrm{MOL}}\right)\right]\left\langle\sqrt{\frac{3}{2}} \sin \beta \cos \beta e^{i \alpha}\right\rangle \\
& +\frac{1}{2}\left[A_{x x}^{\mathrm{MOL}}-A_{y y}^{\mathrm{MOL}}+i\left(A_{x y}^{\mathrm{MOL}}+A_{y x}^{\mathrm{MOL}}\right)\right]\left\langle\sqrt{\frac{3}{8}} \sin ^{2} \beta e^{-i 2 \alpha}\right\rangle \\
& \left.+\frac{1}{2}\left[A_{x x}^{\mathrm{MOL}}-A_{y y}^{\mathrm{MOL}}-i\left(A_{x y}^{\mathrm{MOL}}+A_{y x}^{\mathrm{MOL}}\right)\right]\left\langle\sqrt{\frac{3}{8}} \sin ^{2} \beta e^{i 2 \alpha}\right\rangle\right\} T_{20}
\end{aligned}
$$

where $A_{a b}^{\mathrm{MOL}}(a=x, y, z)$ are the Cartesian components (in the molecular frame) of the spherical tensor $A_{20}^{\mathrm{MOL}}$. Then since NMR Cartesian spatial tensors are sym- 
metric $\left(A_{a b}=A_{b a}\right)$ to first order :

$$
\begin{aligned}
\mathcal{H}= & \frac{2}{3}\left\{\left[A_{z z}^{\mathrm{MOL}}-\frac{1}{2}\left(A_{x x}^{\mathrm{MOL}}+A_{y y}^{\mathrm{MOL}}\right)\right]\left\langle\frac{1}{2}\left(3 \cos ^{2} \beta-1\right)\right\rangle\right. \\
& \left.+\frac{1}{2}\left[A_{x x}^{\mathrm{MOL}}-A_{y y}^{\mathrm{MOL}}\right)\right]\left\langle\frac{3}{2} \sin ^{2} \beta \cos 2 \alpha\right\rangle \\
& +2\left[A_{x y}^{\mathrm{MOL}}\right]\left\langle\frac{3}{2} \sin ^{2} \beta \sin \alpha \cos \alpha\right\rangle \\
& +2\left[A_{x z}^{\mathrm{MOL}}\right]\left\langle\frac{3}{2} \sin \beta \cos \beta \cos \alpha\right\rangle \\
& \left.+2\left[A_{y z}^{\mathrm{MOL}}\right]\left\langle\frac{3}{2} \sin \beta \cos \beta \sin \alpha\right\rangle\right\}\left[\sqrt{\frac{3}{2}} T_{20}\right]
\end{aligned}
$$

where the terms in the angle brackets are the order parameters for the moleculefixed axis system (see Equation 2.3) :

$$
\begin{aligned}
\mathcal{H}= & \frac{2}{3}\left\{\left[A_{z z}^{\mathrm{MOL}}-\frac{1}{2}\left(A_{x x}^{\mathrm{MOL}}+A_{y y}^{\mathrm{MOL}}\right)\right] S_{z z}\right. \\
& +\frac{1}{2}\left[A_{x x}^{\mathrm{MOL}}-A_{y y}^{\mathrm{MOL}}\right] S_{x x-y y} \\
& +2 A_{x y}^{\mathrm{MOL}} S_{x y} \\
& +2 A_{x z}^{\mathrm{MOL}} S_{x z} \\
& \left.+2 A_{y z}^{\mathrm{MOL}} S_{y z}\right\}\left[\sqrt{\frac{3}{2}} T_{20}\right]
\end{aligned}
$$

Using this equation, an experimental quadrupole coupling or anisotropic chemical shift tensor can be used to determine experimentally the order parameter along a given bond in a molecule or dipole couplings can be used to determine order parameters and molecular geometry for rigid molecules $[13,30]$. The next chapter will describe a method for modeling the dipole couplings between protons on flexible molecules and extracting information about molecular conformation and orientation. For dipole couplings $\mathrm{D}_{2 q}^{\mathrm{MOL}}$ has the form :

$$
D_{2 q}^{\mathrm{MOL}}=\sum_{s=-2}^{2} \mathrm{D}_{2 s}^{\mathrm{PAS}} D_{s q}^{2}\left(\Omega_{1}\right)
$$


which for the cylindrically symmetric dipole coupling written in Cartesian coordinates is :

$$
\mathrm{D}_{a b, i j}^{\mathrm{MOL}}=\frac{-\gamma_{1} \gamma_{2} h}{\pi r_{i j}^{3}} \frac{1}{2}\left[3 \frac{r_{a, i j} r_{b, i j}}{\mathbf{r}_{i j}^{2}}-\delta_{a b}\right]
$$

where $\mathbf{r}$ is the internuclear vector between protons $i$ and $j$ and $r_{a, i j}$ is the $a(=x, y, z)$ component of that vector in the molecular frame. 


\section{Chapter 3}

\section{NMR Studies of Molecular}

\section{Conformation and Orientation in}

\section{Liquid Crystals}

\subsection{Introduction}

As discussed in the previous -chapter, second rank interactions in NMR - chemical shift anisotropy, quadrupole couplings, and dipole couplings - can be used to measure order parameters in liquid crystalline systems. Order parameters are important for characterizing anisotropic systems, but they are macroscopic quantities which give no microscopic information about the nature of the molecular interactions responsible for such ordering. Since most anisotropic systems - liquid crystals, polymers, and lipid bilayers, for example - are composed, at least in part, of flexible alkyl chains, an important step in the understanding of intermolecular interactions responsible for ordering in these systems is to characterize not only molecular ori- 
entation, but also molecular conformation.

Previous attempts to study chain conformation and orientation have often employed deuterium NMR spectroscopy $[31,32,33,34,35]$. The deuterium quadrupole couplings measured in these studies provide information about the ordering of individual methylene segments in a flexible chain, but provide no information about the relative positions of different methylene segments in the chain - information crucial for detailed description of chain conformation. A potentially more useful method of measuring the conformation and orientation of alkyl chains in liquid crystals would be to use proton NMR to measure dipole couplings between pairs of protons on the chain. Because of the $\left(3 \cos ^{2} \beta_{12}-1\right) / r_{12}^{3}$ dependence of the dipole couplings, they should be very sensitive to both molecular conformation and orientation.

Proton NMR spectra of molecules in partially ordered systems, however, rapidly become intractably complex with increasing molecular size, making it virtually impossible to extract useful information from the spectra of molecules with more than a few protons. One possible method of simplifying the spectra of such molecules could be to specifically protonate a deuterated molecule at two sites. This would allow easy measurement of the dipole coupling between the two protons but would require the tedious and expensive synthesis of numerous molecules, each specifically protonated at different sites, in order to measure couplings between all pairs of protons on the molecule. An alternative to this is to use random (non-selective) deuteration and a two-dimensional multiple-quantum filtered NMR experiment, as will be described in the next few sections. This method simplifies the synthetic process considerably, requiring only one isotopic substitution reaction; then all the proton dipole couplings can be measured in a single two-dimensional NMR experi- 
ment.

While the dipole couplings can potentially provide a complete description of molecular shape, they cannot be directly interpreted in terms of molecular conformation and orientation, since the NMR experiment measures couplings that are averaged over the rapid molecular motions that occur in solution. Thus the dipole couplings are averaged over all conformations and orientations sampled by a molecule. To obtain useful information about conformation and orientation, the couplings must be interpreted using models for intra- and inter-molecular interactions.

Before applying this method to complicated systems of interest like liquid crystals, polymers, or lipid bilayers, we will start with simple model systems. A series of $\mathrm{n}$-alkanes (hexane through decane) dissolved in a nematic liquid crystal provides a set of good model systems on which to test the experimental method for measuring dipole couplings between pairs of protons on flexible molecules. The detailed information about molecular conformation and orientation obtained from the experimentally measured proton dipole couplings can then be used to test several models for intermolecular interactions in nematic liquid crystals.

\subsection{Two-Proton Filtered COSY Experiment}

A conventional one-dimensional NMR spectrum of fully protonated $n$-hexane dissolved in EK11650 is shown in Figure 3.1. The spectrum is very complex and would be virtually impossible to analyze to obtain dipole couplings between pairs of protons on the molecule. To simplify the spectrum, the alkanes will be randomly deuterated to a level of approximately $80 \%$. With $80 \%$ of the protons replaced by 
deuterons, a large number of hexane molecules will have only two protons left on them, as shown in Figure 3.2. If the signal from molecules with two protons can be selectively retained while discarding the signal from all other hexane molecules, then a simple spectrum containing pairs of lines whose splittings give the dipole couplings between pairs of protons on $n$-hexane can be obtained. As has been previously discussed $[36,37,38]$ a two-proton filtered COSY experiment will allow us to do this. The pulse sequence for this experiment is :

$$
\left(\frac{\pi}{2}\right)_{\phi}-\frac{t_{1}}{2}-(\pi)_{\phi}-\frac{t_{1}}{2}-\left(\frac{\pi}{2}\right)_{\phi}-\frac{\tau_{1}}{2}-(\pi)_{x}-\frac{\tau_{1}}{2}-\left(\frac{\pi}{2}\right)_{x}-\frac{\tau_{2}}{2}-(\pi)_{x}-\frac{\tau_{2}}{2}-t_{2}
$$

This pulse sequence begins with a $\frac{\pi}{2}$ pulse followed by $t_{1}$ evolution. A $\pi$ pulse during $t_{1}$ is used to refocus chemical shift, and then a second $\frac{\pi}{2}$ pulse is used to generate multiple-quantum coherence which evolves during $\tau_{1}$. If a molecule has only one proton on it, multiple-quantum coherence cannot be generated at this point, and signal from such molecules is lost. The multiple-quantum coherence evolves during $\tau_{1}$ while chemical shift is refocussed by the $\pi$ pulse in the middle. Then the multiple-quantum coherence is converted to observable single-quantum signal by the last $\frac{\pi}{2}$ pulse and detection is done after an echo during $\tau_{2}$. The echo allows the fast-relaxing liquid crystal proton signal to decay, while refocussing the signal from the protons on the alkane. Signal from molecules with three or more protons is removed by co-adding numerous experiments, each with a different value of $\tau_{1}$. This works because double-quantum coherences from two-spin systems don't evolve in time while double-quantum coherences from three or more spin systems do evolve. By co-adding experiments with different values of $\tau_{1}$, the double-quantum signal from molecules with more than two protons will average to zero.

Looking at this experiment in the density operator formalism for the two dipole- 


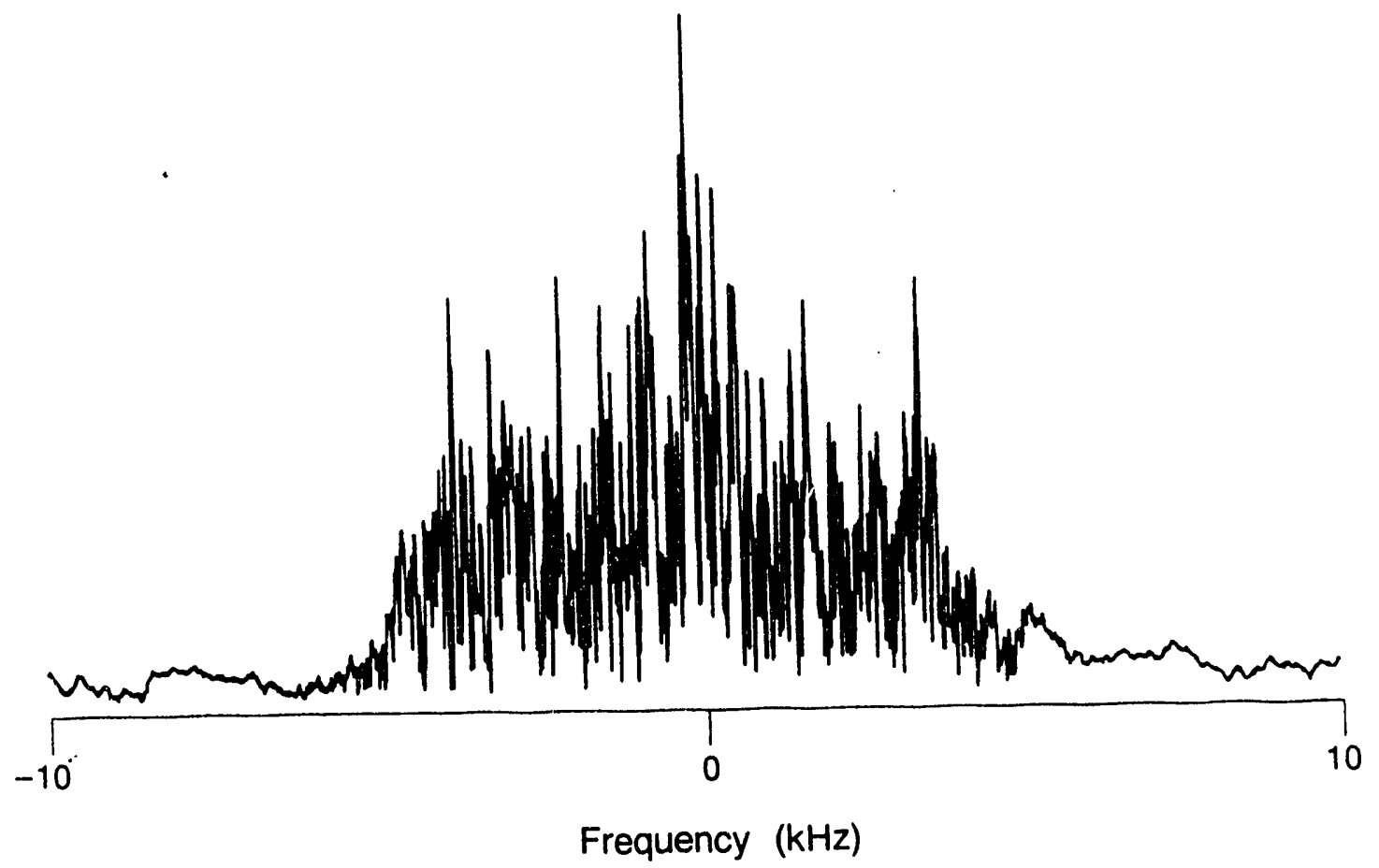

XBL $8610 \cdot 11728$

Figure 3.1: A conventional one-dimensional NMR spectrum of $n$-hexane dissolved in EK 11650. 


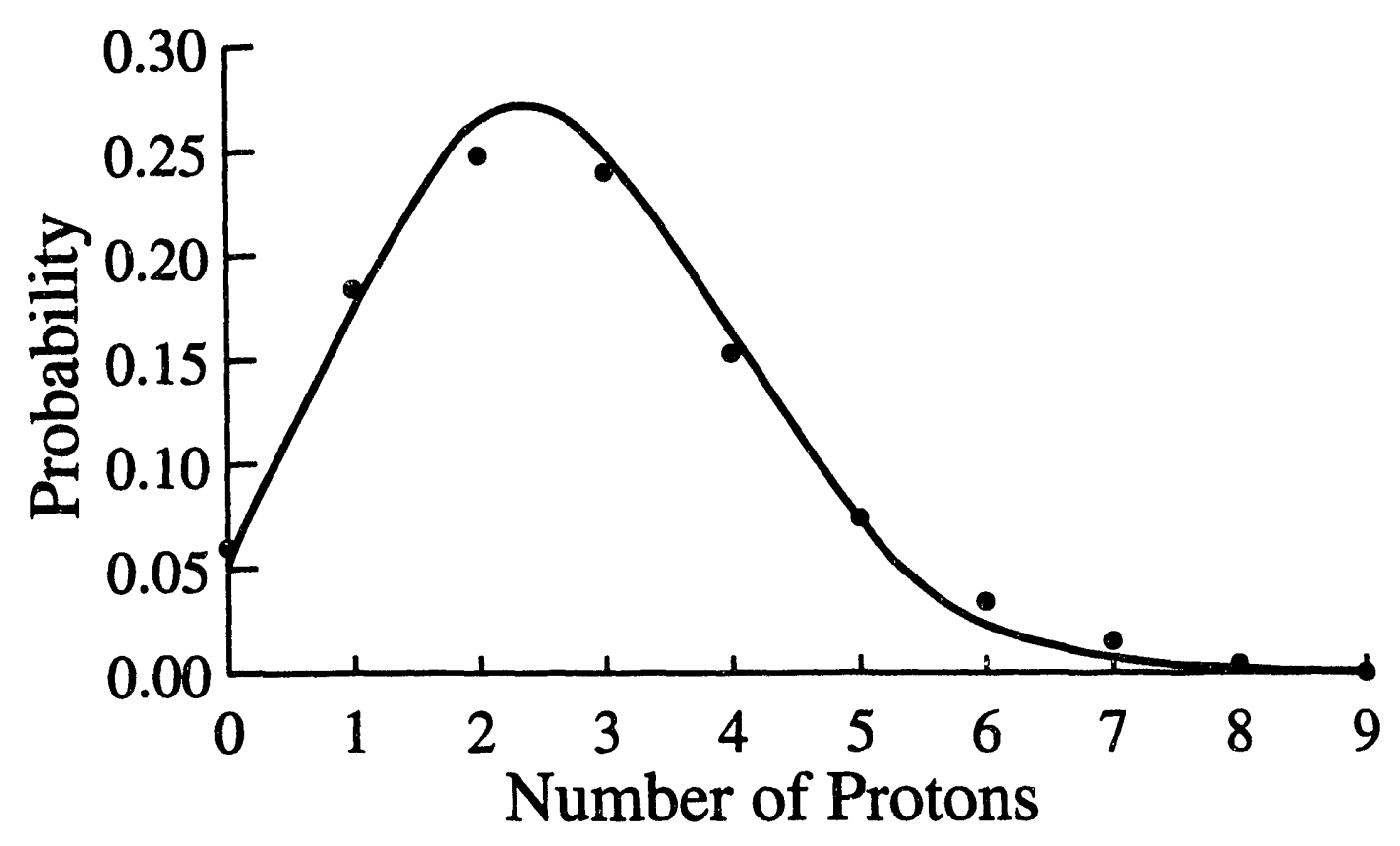

Figure 3.2: The distribution of protonated isomers of $n$-hexane deuterated to a level of 0.81 . The solid line is the theoretical curve if the deuteration is truly random, and the points $(\bullet)$ are the results from a mass spectrum. 
coupled spins $\frac{1}{2}$ from the previous chapter, the signal from a single experiment with $\phi=x$ can be written :

$$
\begin{gathered}
S(t)=\operatorname{Tr}\left(e^{-i \mathcal{H} t_{2}} V e^{-i \mathcal{H} \frac{t_{1}}{2}} e^{-i \pi I_{x}} e^{-i \mathcal{H} \frac{t_{1}}{2}} e^{-i \frac{\pi}{2} I_{x}} \rho_{\mathrm{eq}}\right. \\
\left.e^{i \frac{\pi}{2} I_{x}} e^{i \mathcal{H} \frac{t_{1}}{2}} e^{i \pi I_{x}} e^{i \mathcal{H} \frac{t_{1}}{2}} V^{\dagger} e^{i \mathcal{H} t_{2}} I^{+}\right)
\end{gathered}
$$

where

$$
\begin{aligned}
V & =e^{-i \mathcal{H} \frac{\tau_{2}}{2}} e^{-i \pi I_{x}} e^{-i \mathcal{H} \frac{\tau_{2}}{2}} e^{-i \frac{\pi}{2} I_{x}} e^{-i \mathcal{H} \frac{\tau_{1}}{2}} e^{-i \pi I_{x}} e^{-i \mathcal{H} \frac{\tau_{1}}{2}} e^{-i \frac{\pi}{2} I_{x}} \\
V^{\dagger} & =e^{i \frac{\pi}{2} I_{x}} e^{i \mathcal{H} \frac{\tau_{1}}{2}} e^{i \pi I_{x}} e^{i \mathcal{H} \frac{\tau_{1}}{2}} e^{i \frac{\pi}{2} I_{x}} e^{i \mathcal{H} \frac{\tau_{2}}{2}} e^{i \pi I_{x}} e^{i \mathcal{H} \frac{\tau_{2}}{2}}
\end{aligned}
$$

are the mixing propagator and its adjoint. Rearranging the trace, expanding in the basis of eigenstates of the Hamiltonian and evaluating the effect of the first $\frac{\pi}{2}$ pulse on the equilibrium density operator, this becomes :

$$
\begin{array}{r}
S(t)=\sum_{i, j, k, l, m, n}\left\langle i\left|V e^{-i \mathcal{H} \frac{t_{1}}{2}}\right| l\right\rangle\left\langle l\left|e^{-i \pi I_{x}} e^{-i \mathcal{H} \frac{t_{1}}{2}}\right| k\right\rangle\left\langle k\left|-I_{y} e^{i \mathcal{H} \frac{t_{1}}{2}}\right| n\right\rangle \\
\left\langle n\left|e^{i \pi I_{x}} e^{i \mathcal{H} \frac{t_{1}}{2}}\right| m\right\rangle\left\langle m\left|V^{\dagger} e^{i \mathcal{H} t_{2}}\right| j\right\rangle\left\langle j\left|I^{+} e^{-i \mathcal{H} t_{2}}\right| i\right\rangle
\end{array}
$$

Since the basis states are eigenstates of the Hamiltonian, this can be written :

$$
\begin{aligned}
S(t)= & \sum_{i, j, k, l, m, n} e^{i\left(\omega_{j}-\omega_{i}\right) t_{2}} e^{i\left(\left(\omega_{m}+\omega_{n}\right)-\left(\omega_{k}+\omega_{l}\right)\right) \frac{t_{1}}{2}} \\
& \langle i|V| l\rangle\left\langle l\left|e^{-i \pi I_{x}}\right| k\right\rangle\left\langle k\left|-I_{y}\right| n\right\rangle\left\langle n\left|e^{i \pi I_{x}}\right| m\right\rangle\left\langle m\left|V^{\dagger}\right| j\right\rangle\left\langle j\left|I^{+}\right| i\right\rangle
\end{aligned}
$$

where $\omega_{\alpha}$ are the eigenvalues of the Hamiltonian, given in Figure 1.3. The frequencies which occur in the spectrum are the same as in Figure 1.5 but the intensities are slightly different because of the additional delay $\tau_{2}$ and the $\pi$ pulse in $\tau_{2}$.

\subsection{Experimental Details}

The alkanes to be studied ( $n$-hexane, $n$-heptane, $n$-octane, $n$-nonane, and $n$-decane) were randomly deuterated to a level of $0.8-0.9$ by gas-phase exchange with $D_{2}$ over 
a $\mathrm{Pd}$ catalyst at $90^{\circ} \mathrm{C}$. The level of deuteration was determined by mass spectrometry. Five samples, each containing one of the n-alkanes, were prepared by dissolving a sufficient amount of the randomly deuterated alkane in the liquid crystal EK 11650 (p-pentylphenyl-2-chloro-(4-benzylbenzoyloxy)-benzoate) to bring the concentration of the alkane to 30 mole \%. The NMR experiments were performed on a home-built $360 \mathrm{MHz}$ spectrometer using the pulse sequence in Equation 3.1. For each sample, $1024 t_{2}$ points and $384 t_{1}$ points were collected with a $12 \mathrm{kHz}$ sweep width in both dimensions. FrI each $t_{1}$ point 96 scans were averaged, cycling phase $\phi$ through $0^{\circ}, 90^{\circ}, 180^{\circ}$, and $270^{\circ}$ every scan, cycling the receiver through $0^{\circ}$ and $180^{\circ}$ every scan, and incrementing $\tau_{1}$ by $1.4 \mathrm{~ms}$ every fourth scan from a starting value of $1.4 \mathrm{~ms} . \tau_{2}$ was kept fixed at a value of $1.2 \mathrm{msec}$. Double-quantum cieuterium decoupling [39] over a $30 \mathrm{kHz}$ bandwidth was used throughout the experiment to remove proton-deuteron dipole couplings. The spectra were recorded at a reduced temperature $\left(\mathrm{T}_{\mathrm{R}}=\mathrm{T} / \mathrm{T}_{N I}\right)$ of 0.875 to allow direct comparison of the results for different alkanes. The experimental temperatures and clearing points for the different samples are given in Table 3.1.

Table 3.1: Experimental temperatures, clearing points and reduced temperatures for the $n$-alkane/EK11650 samples.

\begin{tabular}{|c||r|r|r|}
\hline & $\begin{array}{c}\text { Experimental } \\
\text { Temperature }(\mathrm{T})\end{array}$ & $\begin{array}{c}\text { Clearing } \\
\text { Point }\left(\mathrm{T}_{\mathrm{NI}}\right)\end{array}$ & $\begin{array}{r}\text { Reduced Temperature } \\
\mathrm{T}_{\mathrm{R}}=\frac{\mathrm{T}}{\mathrm{T}_{\mathrm{NI}}}\end{array}$ \\
\hline \hline hexane & 314.1 & 359.0 & 0.875 \\
heptene & 309.7 & 354.0 & 0.875 \\
octane & 308.2 & 352.2 & 0.875 \\
nonane & 306.9 & 350.8 & 0.875 \\
decane & 304.8 & 348.4 & 0.875 \\
\hline
\end{tabular}

Since the dipole couplings are much larger in magnitude than the differences in chemical shift for most of the proton pairs on these alkanes, usually only four peaks 
appear in the spectra with significant intensity. These appear in square patterns, from which the magnitude of the dipole couplings can be determined (neglecting $\mathrm{J}$ couplings which are not resolved in the spectra) by the relation :

$$
|D|=\frac{4}{3} s-\frac{2}{3} \sqrt{s^{2}+3(\delta \omega)^{2}}
$$

where $s$ is the length of the side of the square and $\delta \omega$ is the difference in chemical shift between the two coupled protons. Increasing molecular size precludes the measurement and assignment of all dipole couplings. Fortunately, the readily resolvable couplings are those which provide the greatest constraints on molecular conformation : couplings between geminal protons, couplings involving a methyl proton, and couplings between methylene protons which are on second-nearest-neighbor carbons.

Assignment of the couplings to specific pairs of protons is based primarily on the chemical shifts of coupled protons, but for longer alkanes also relies on comparison with the results for $n$-hexane $[36,37,38]$. The sign of each coupling can be determined based on the assumption that the alkanes are aligned predominantly along the nematic director - along the applied magnetic field. This means that the geminal proton couplings, with their internuclear vector approximately perpendicular to the applied magnetic field, are positive, and couplings between proton pairs whose internuclear vector lies along the length of the chain are negative.

Figures 3.3, 3.4, and 3.5 show an example of the analysis of the two-protonfiltered COSY spectrum of $n$-decane. The square patterns in the spectrum and their assignment to pairs of protons on the molecule are shown, with the labeling of protons given in Figure 3.6. Table 3.2 lists the dipole couplings obtained from this spectrum, as well as those obtained from the spectra of the other $n$-alkanes in this 


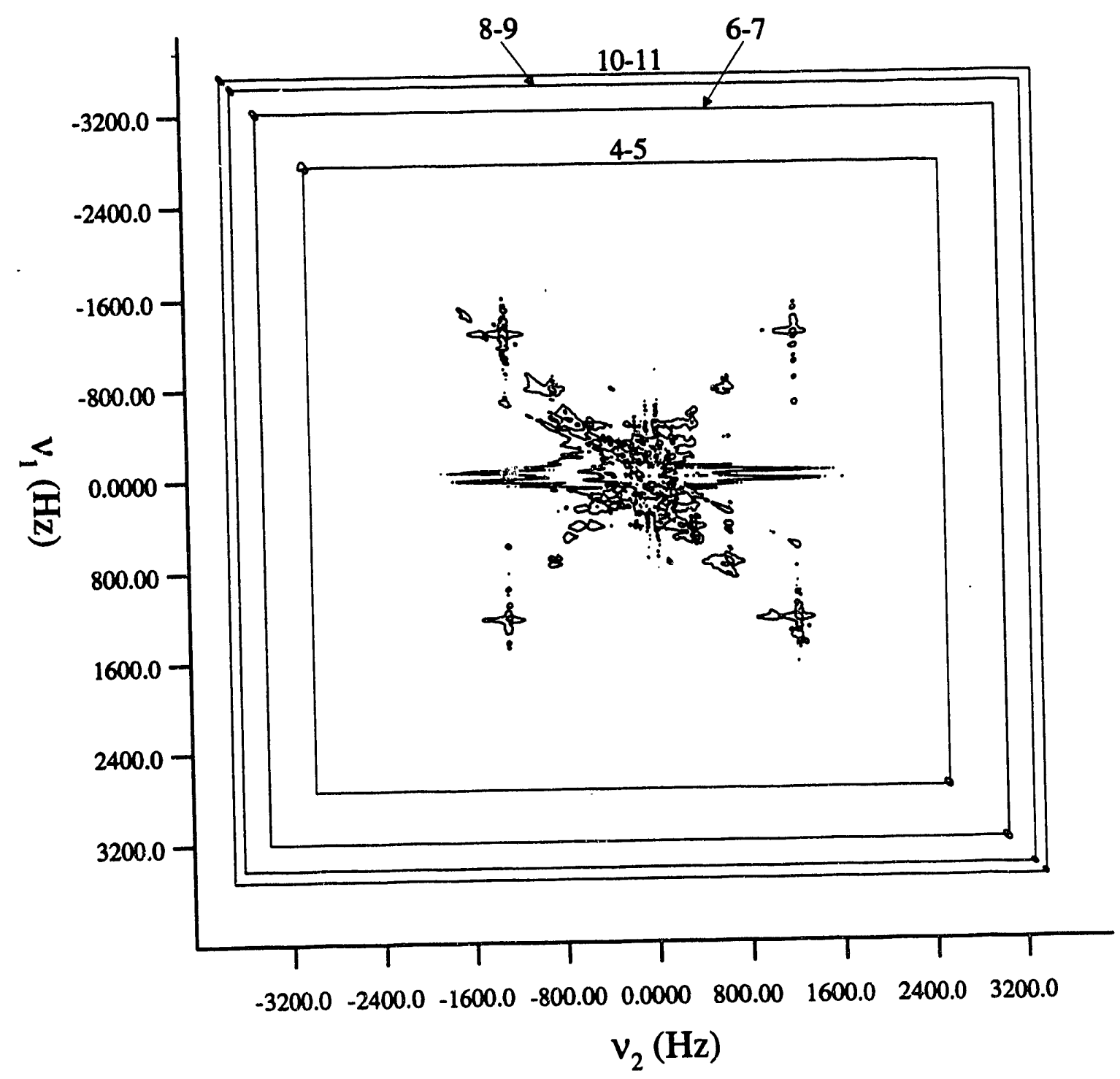

Figure 3.3: The two-proton-filtered CDSY spectrum of $n$-decane dissolved in EK 11650 with the square patterns of peaks in the spectra labeled by the protons corresponding to those peaks. The numbering of the protons is given in Figure 3.6. 


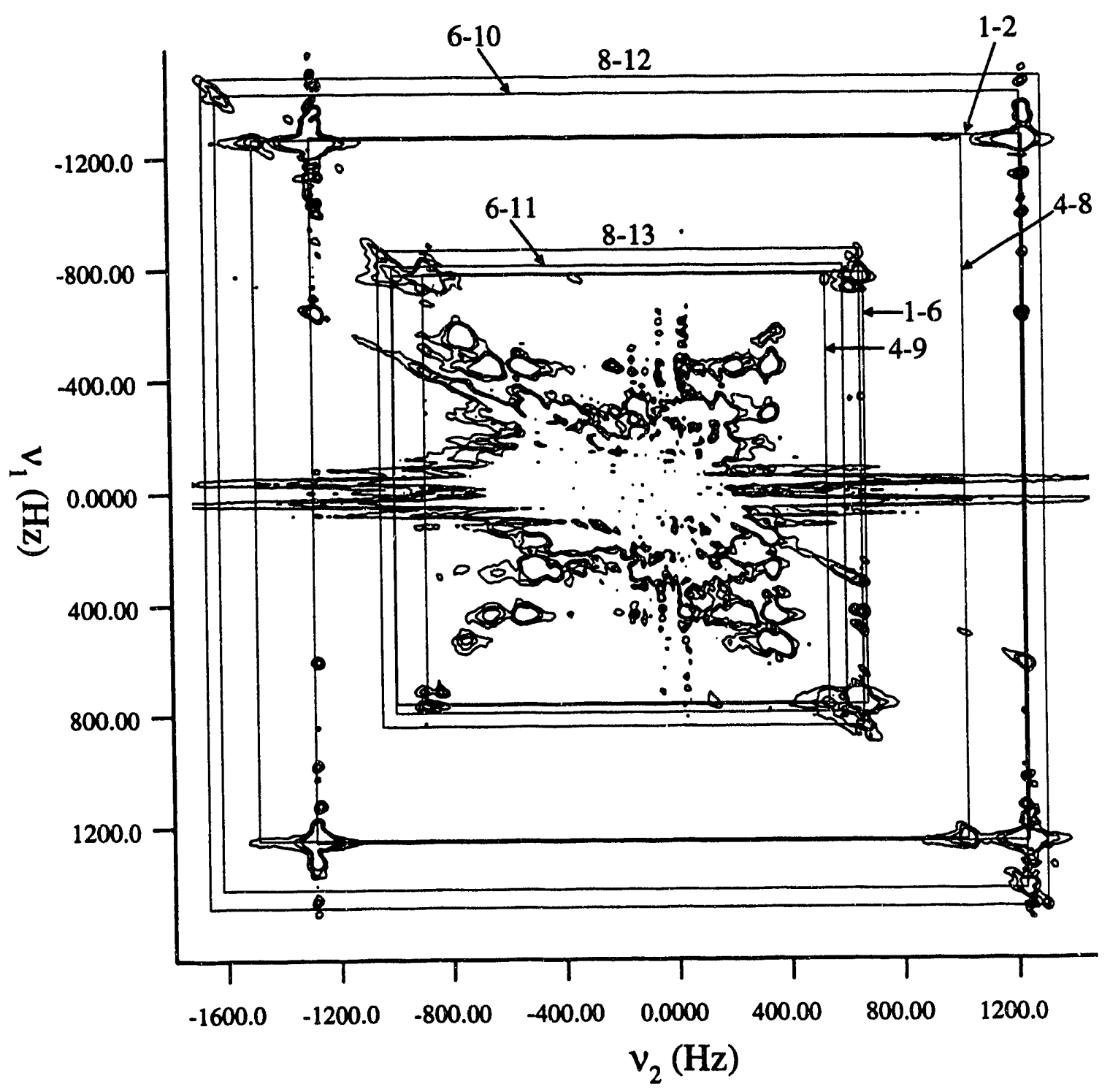

Figure 3.4: Enlarged view of the two-proton-filtered COSY spectrum of $n$ decane dissolved in EK 11650. 


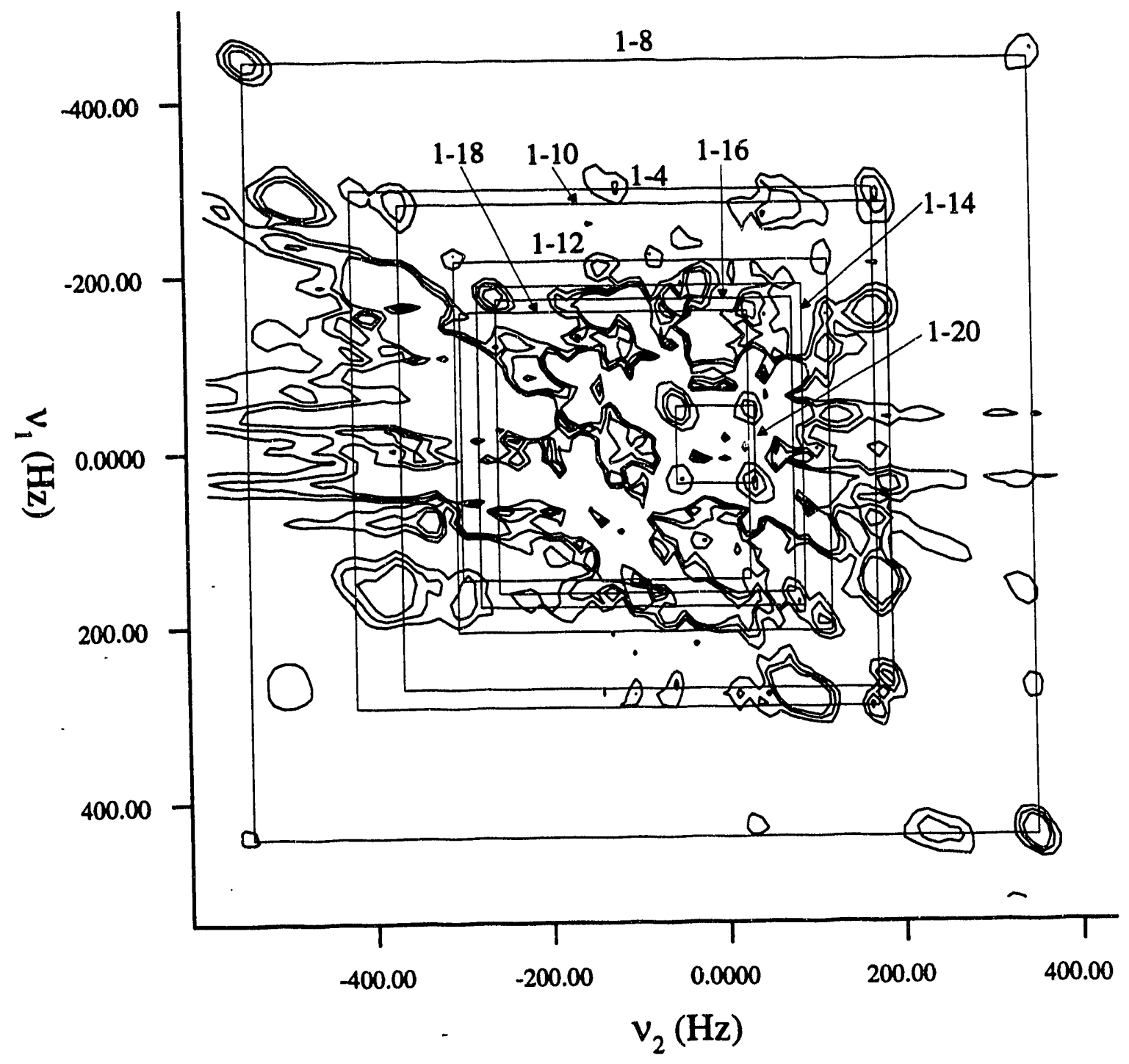

Figure 3.5: Central portion of the two-proton-filtered COSY spectrum of $n$ decane dissolved in EK 11650. 


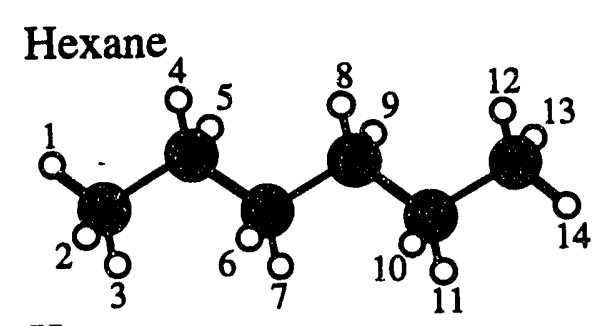

Heptane

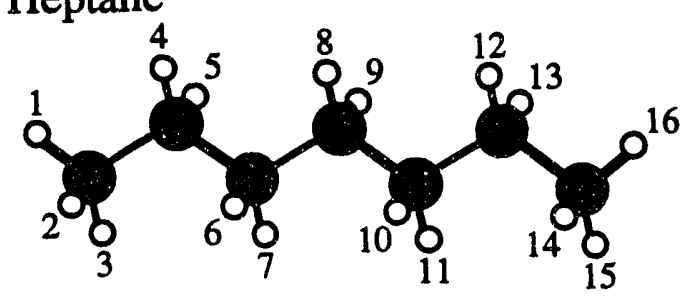

Octane

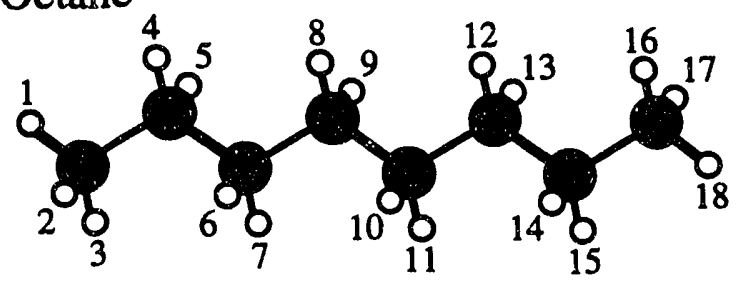

Nonane

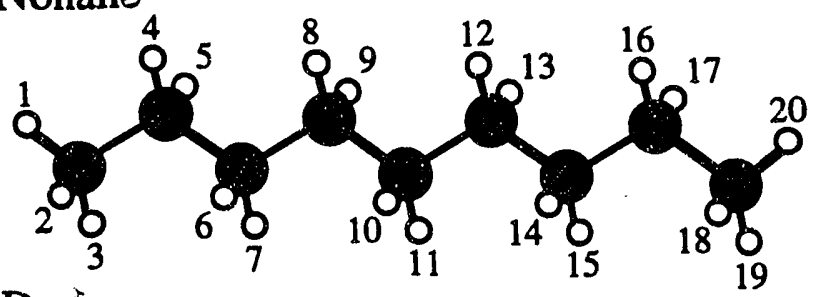

Decane

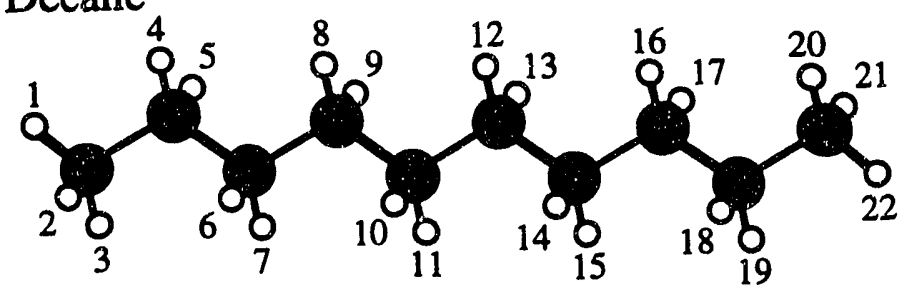

Figure 3.6: The numbering of the protons on the alkanes. The even-numbered protons are on the same side of the molecule, coming out of the page, and the odd-numbered protons are on the side of the molecule going into the page. 
study, and Table 3.3 lists the chemical shifts of the protons on the alkanes which were used to calculate the dipole couplings from Equation 3.6.

Table 3.2: Experimental dipole couplings.

\begin{tabular}{|c||r|r|r|r|r|}
\hline \multicolumn{1}{|c||}{} & \multicolumn{5}{c|}{ Dipole Coupling $(\mathrm{Hz})^{\ddagger}$} \\
\cline { 2 - 6 } Protons $^{\dagger}$ & Hexane & Heptane & Octane & Nonane & Decane \\
\hline \hline $1-2$ & 1526 & 1489 & 1637 & 1546 & 1669 \\
$4-5$ & 3288 & 3461 & 3539 & 3570 & 3660 \\
$6-7$ & 3711 & 3897 & 4119 & 4104 & 4283 \\
$8-9$ & & 4093 & 4319 & 4420 & 4577 \\
$10-11$ & & & & 4420 & 4697 \\
$1-4$ & -310 & -226 & -319 & -265 & -313 \\
$1-6$ & -852 & -916 & -949 & -958 & -998 \\
$1-8$ & -484 & -520 & -538 & -539 & -556 \\
$1-10$ & -272 & -290 & -296 & -300 & -319 \\
$1-12$ & -167 & -192 & -204 & -206 & -219 \\
$1-14$ & & -127 & -129 & -138 & -151 \\
$1-16$ & & & -96 & -93 & -127 \\
$1-18$ & & & & -73 & -82 \\
$1-20$ & & & & & -57 \\
$4-8$ & -1323 & -1453 & -1544 & -1554 & -1666 \\
$4-9$ & -881 & -924 & -976 & -977 & -1020 \\
$6-10$ & & -1575 & -1710 & -1721 & -1863 \\
$6-11$ & & -975 & -1088 & -1028 & -1062 \\
$8-12$ & & & & -1779 & -1970 \\
$8-13$ & & & & -1097 & -1140 \\
\hline
\end{tabular}

${ }^{\dagger}$ Labeling of protons is given in Figure 3.6

${ }_{\ddagger}$ Experimental Error $\pm 25 \mathrm{~Hz}$

\subsection{Experimental Dipole Couplings}

While it hasn't been possible to determine all of the dipole couplings between pairs of protons on the $n$-alkanes, a significant number have been determined, as shown in Table 3.4. Trends in the experimental dipole couplings listed in Table 3.2 are 
Table 3.3: Experimental chemical shifts.

\begin{tabular}{|c||r|r|r|r|r|}
\hline \multicolumn{1}{|c||}{} & \multicolumn{5}{c|}{ Chemical Shift (Hz) } \\
\cline { 2 - 6 } Protons $^{\dagger}$ & Hexane & Hentane & Octane & Nonane & Decane \\
\hline \hline $1,2,3$ & 0 & 0 & 0 & 0 & 0 \\
4,5 & 219 & 227 & 229 & 230 & 230 \\
6,7 & 185 & 186 & 190 & 189 & 187 \\
8,9 & & 180 & 177 & 178 & 174 \\
10,11 & & & & 178 & 165 \\
\hline
\end{tabular}

${ }^{\dagger}$ Labeling of protons is given in Figure 3.6

more easily seen if the individual couplings are plotted versus chain length, as in Figures 3.7, 3.8, and 3.9. Figure 3.7 shows the geminal couplings as a function of chain length. One notable feature is the even-odd variation of the magnitude of the methyl proton couplings. This has been observed previously in a deuterium NMR study of alkyl chains dissolved in liquid crystals [40] and reflects the difference in the shape of even and odd alkanes. If we consider the conformations of an alkane as symmetrical fluctuations about the lowest-energy, all-trans conformation, then, on average, even alkanes have $C_{2 h}$ symmetry and odd alkanes have $C_{2 v}$ symmetry. Looking at hexane and heptane in the all-trans conformation, as in Figure 3.10, it can be seen that the two terminal carbon-carbon bonds in hexane are parallel to each other and the terminal carbon-carbon bonds in heptane are at an angle of $112^{\circ}$ to each other. If we assume that the liquid crystal aligns individual carbon-carbon bonds or that the inertia tensor of the molecule determines its lowest energy orientation, then even $n$-alkanes will have these terminal carbon-carbon bonds aligned, on average, closer to the nematic director than the terminal carbon-carbon bonds in odd n-alkanes. This makes the geminal methyl proton coupling larger for even $n$-alkanes than for odd $n$-alkanes.

The remaining proton dipole couplings measured in this study are shown in 


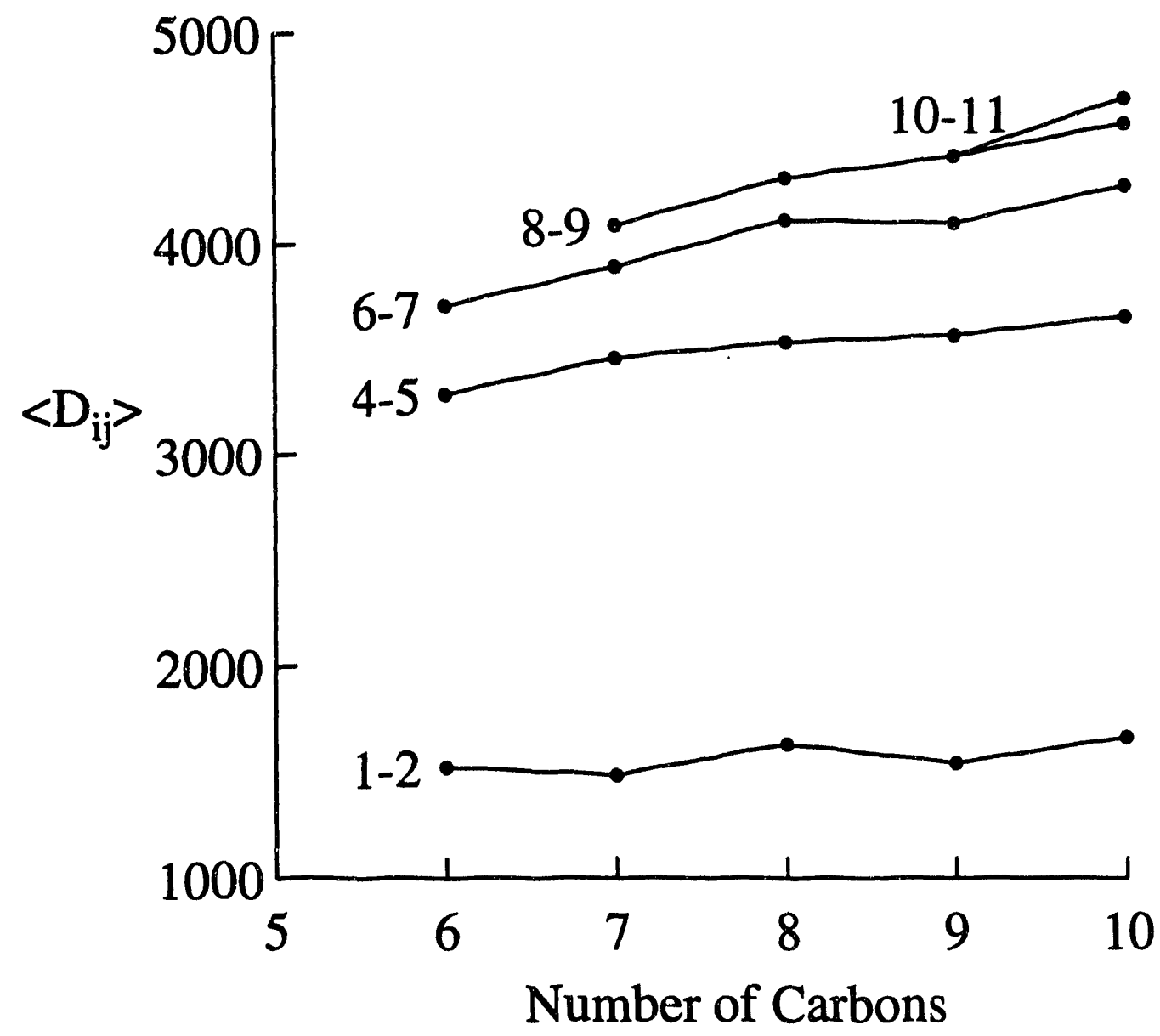

Figure 3.7: Geminal proton dipole couplings as a function of chain length. 


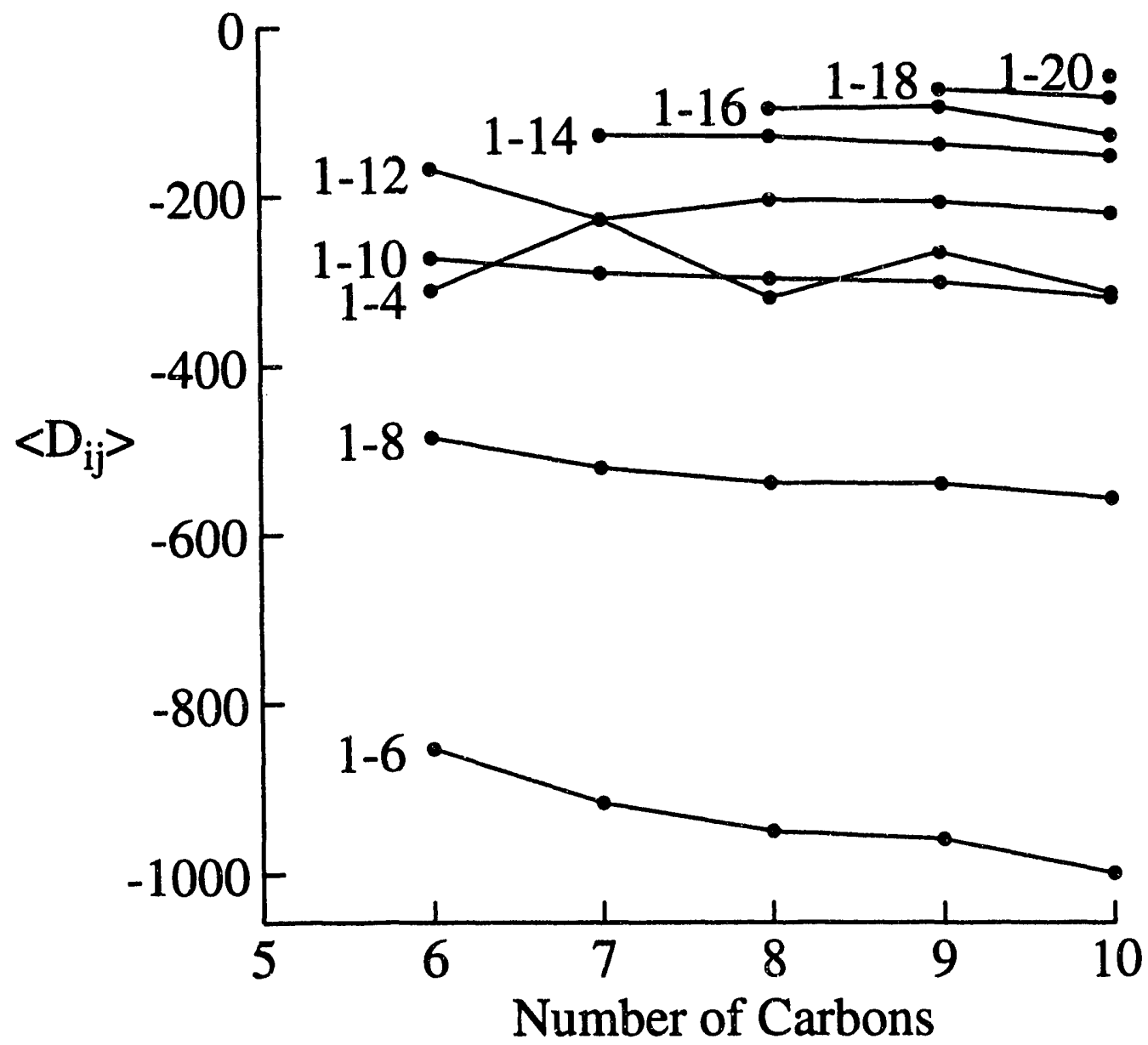

Figure 3.8: Proton dipole couplings involving a methyl proton as a function of chain length. 


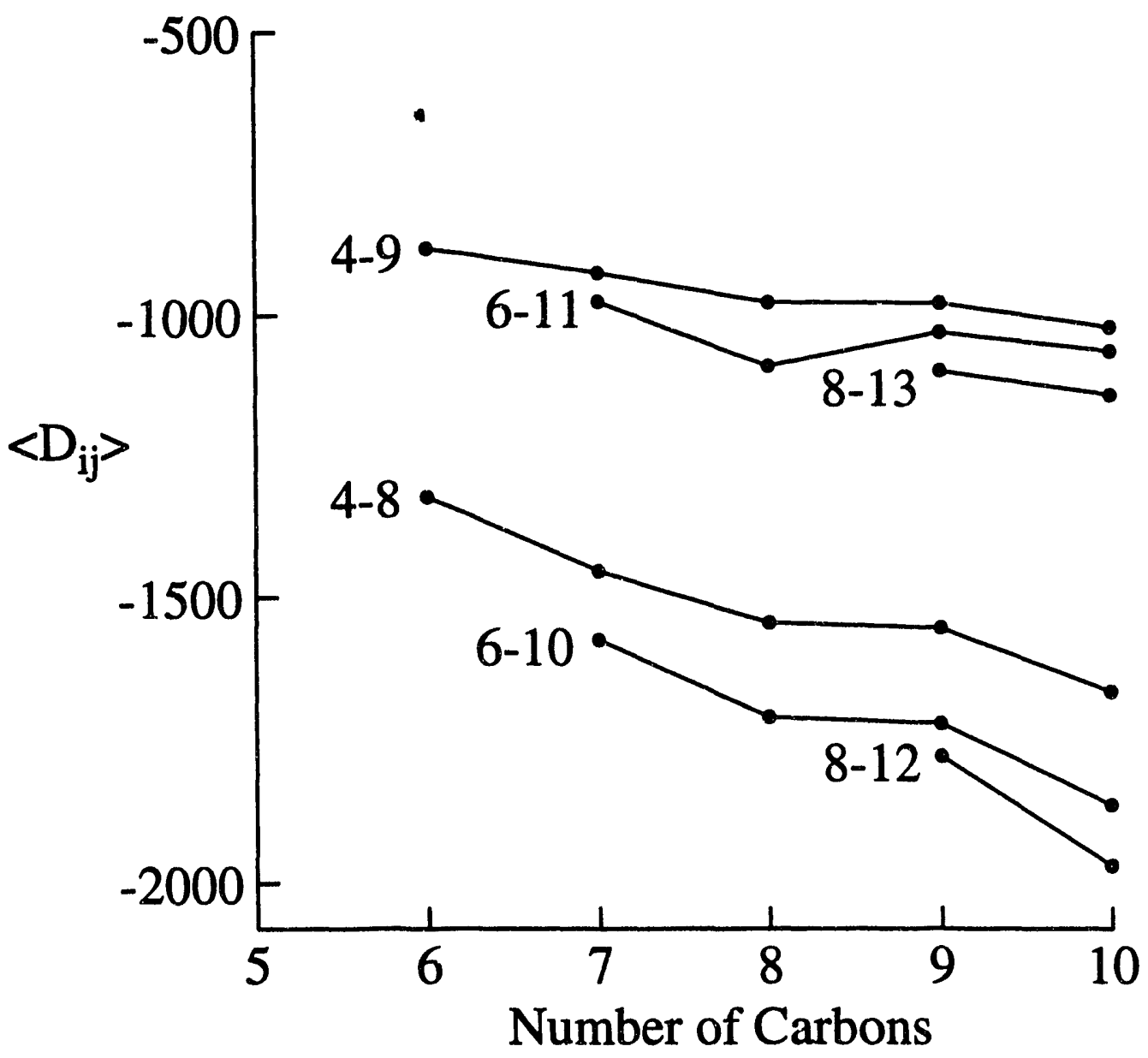

Figure 3.9: Proton dipole couplings between protons on second-nearestneighbor carbons as a function of chain length. 

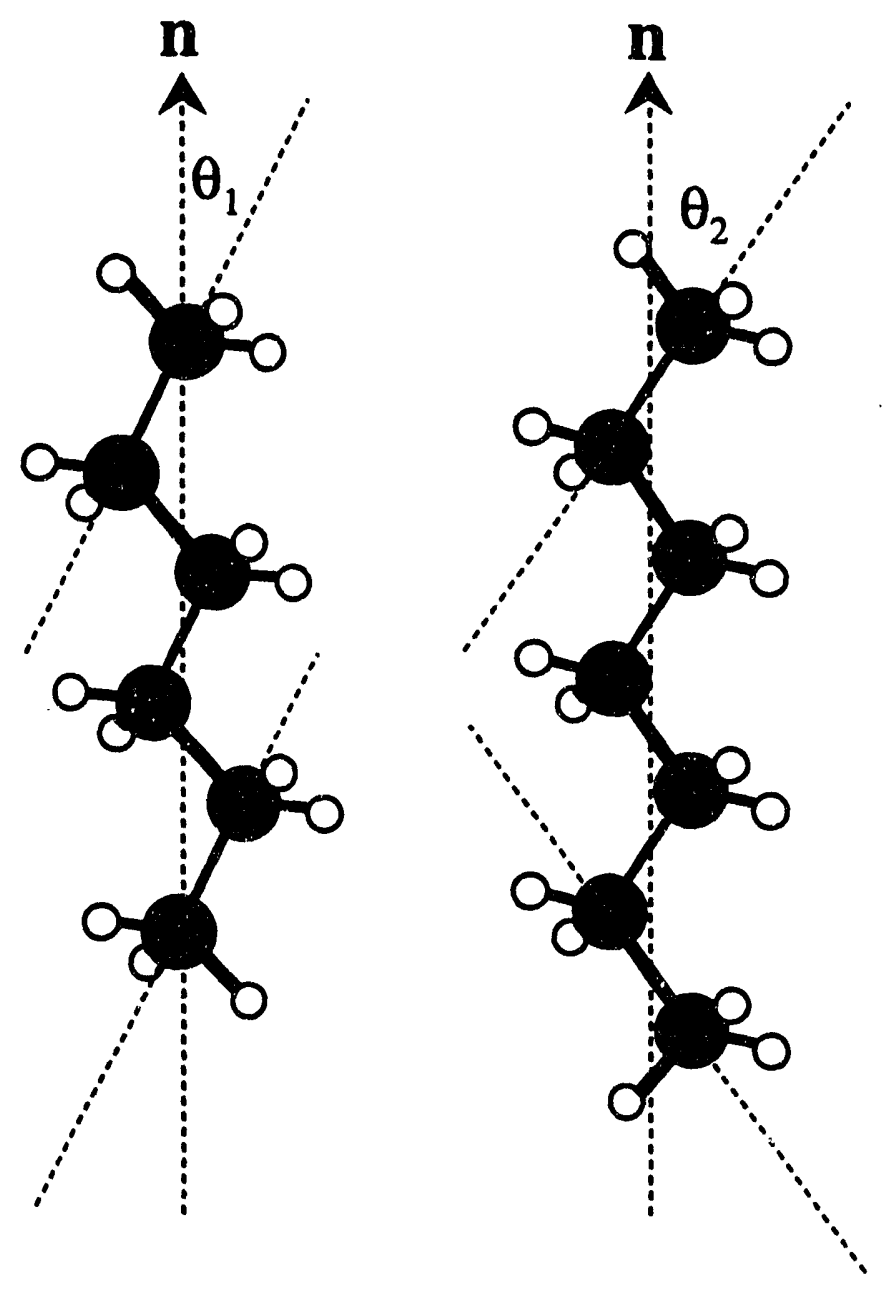

Figure 3.10: The even-odd variation of the dipole couplings between proton pairs 1-2 and 1-4 can be pictured by considering the lowest energy, alltrans conformations of hexane and heptane. If it is assumed that the nematic mean field attempts to align carbon-carbon bonds or the inertia tensor of the molecule, then the even alkanes with their terminal carbon-carbon bonds parallel to each other will have these bonds aligned closer to parallel to the nematic director (and the applied magnetic field) than in the odd alkanes, whose terminal carbon-carbon bonds are at an angle of $112^{\circ}$ to each other. This makes $\theta_{1}$ smaller than $\theta_{2}$ and the dipole couplings between proton pairs 1-2 and 1-4 larger in magnitude in the even alkanes than in the odd alkanes. 
Table 3.4: Number of experimental dipole couplings for the $n$-alkanes.

\begin{tabular}{|c||r|r|}
\hline & $\begin{array}{c}\text { Number of Dipole } \\
\text { Couplings Used }\end{array}$ & $\begin{array}{c}\text { Total Number of } \\
\text { Dipole Couplings }\end{array}$ \\
\hline \hline hexane & 10 & 16 \\
heptane & 14 & 22 \\
octane & 15 & 29 \\
nonane & 19 & 37 \\
decane & 20 & 46 \\
\hline
\end{tabular}

Figures 3.8 and 3.9. These couplings give information about molecular shape that is not available from deuterium quadrupole couplings. Figure 3.8 shows the dipole couplings between a methyl proton and protons on other carbon atoms in the chain. The even-odd variation in the magnitude of the coupling between a methyl proton and a proton on the neighboring carbon (protons 1 and 4) can be seen. This is again the result of the difference in ordering of terminal carbon-carbon bonds in even and odd $n$-alkanes. The effect of the $\left(3 \cos ^{2} \beta-1\right)$ dependence of the dipole couplings can also be seen. Despite the proximity of proton 4 to the methyl protons, the coupling between this proton and the methyl protons is smaller than the coupling between the methyl protons and proton 6 . This is because the internuclear vector between the methyl protons and proton 4 is closer, on average, to the magic angle $\left(54.7^{\circ}\right)$ with respect to the applied magnetic field, than the internuclear vector between the methyl protons and proton 6 , which is approximately parallel to the applied magnetic field. The more distant couplings reflect the dominance of the $r^{-3}$ dependence of the dipole couplings - the magnitude of couplings to the methyl protons decreases as the distance of its coupling partner increases.

Figure 3.9 shows the couplings between protons on second-nearest-neighbor carbon atoms. There appears to be an even-odd variation in the couplings, which again 
can be attributed to the difference in ordering of even- and odd-length chains, as pictured in Figure 3.10. Internuclear vectors between protons on second-nearestneighbor carbons in the all-trans conformation of odd $n$-alkanes are closer to parallel to the nematic director (and the applied magnetic field) than those in even $n$-alkanes. This makes the couplings in odd $n$-alkanes larger than in even $n$-alkanes.

\subsection{Modeling of Alkanes}

The proton dipole couplings listed in Table 3.2 do not directly provide quantitative information about molecular conformation and orientation, but instead only give constraints on the ensemble-averaged conformation and orientation of the n-alkanes. To obtain quantitative information, computer simulation of the averaging process must be carried out using suitable models for inter- and intra-molecular interactions. One method assumes a mean-field potential, $U(n, \Omega)$, for the energy of a single solute molecule in a uniaxial nematic solvent. $U(n, \Omega)$ is a function of the discrete conformations, $n$, of the solute molecule and the orientation, $\Omega$, of a solute moleculefixed axis system with respect to the nematic director. The averaged dipole coupling between two protons on this molecule can be expressed as :

$$
\left\langle\mathrm{D}_{12}\right\rangle=\frac{2}{3} \sum_{n} p^{n} \sum_{\alpha \beta} S_{\alpha \beta}^{n} \cdot \mathrm{D}_{\alpha \beta, 12}^{\mathrm{MOL}, n}
$$

where $p^{n}$ is the probability of conformer $n, S_{\alpha, \beta}^{n}$ is the order tensor for the molecule in that conformation, and $D_{\alpha \beta, 12}^{\mathrm{MOL}, n}$ is the dipole coupling tensor in the molecular frame for the two protons with the molecule in conformation $n$. The order tensor is defined as :

$$
S_{\alpha, \beta}^{n}=\frac{1}{2}\left\langle 3 \cos \theta_{\alpha} \cos \theta_{\beta}-\delta_{\alpha \beta}\right\rangle
$$


where $\theta_{\chi}(\chi=x, y, z)$ is the angle between the director and the molecular $\chi$ axis. The dipole coupling tensor for two spins $\frac{1}{2}$ in conformer $n$ in the molecular frame is :

$$
\mathrm{D}_{\alpha \beta, 12}^{\mathrm{MOL}, n}=-\frac{\gamma_{1} \gamma_{2} h}{2 \pi r_{12}^{3}}\left(3 \cos \theta_{\alpha, 12} \cos \theta_{\beta, 12}-\delta_{\alpha \beta}\right)
$$

where $\theta_{\chi, 12}$ is the angle between the internuclear vector $\mathbf{r}_{12}$ and the molecular $\chi$ axis. Since $p^{n}$ and $S_{\alpha, \beta}^{n}$ are average quantities and depend on $U(n, \Omega)$, a good choice of $U(n, \Omega)$ will allow one to simulate the experimental dipole couplings and interpret them in terms of molecular conformation and orientation.

In order to simplify the modeling of $U(n, \Omega)$, the solute conformer energy is assumed to be independent of its orientation. The potential $U(n, \Omega)$ can then be separated into internal (solvent-independent) and external (solute-solvent) terms [41] :

$$
U(n, \Omega)=U_{\text {int }}(n)+U_{\text {ext }}(n, \Omega)
$$

The internal part depends only on conformation and will be modeled by the Rotational Isomeric States (RIS) model [42] where rotation about each carbon-carbon bond is restricted tc three discrete states (trans, gauche ${ }^{+}$, and gauche ${ }^{-}$) :

$$
U_{\text {int }}(n)=n_{\mathrm{g}} E_{\mathrm{g}}+n_{\mathrm{g}+\mathrm{g}}-E_{\mathrm{g}^{+} \mathrm{g}^{-}}
$$

In the first term, $n_{\mathrm{g}}$ represents the number of gauche bonds in conformer $n$, and $E_{\mathrm{g}}$ the gas phase trans-gauche energy differerce (approximately $3.8 \mathrm{~kJ} / \mathrm{mol}$ [43]). The second term penalizes conformations with adjacent gauche ${ }^{+}$and gauche ${ }^{-}$bonds (the pentan $\epsilon$ effect [42]), assuming the energy of this configuration, $E_{\mathrm{g}^{\dagger} \mathrm{g}^{-}}$, is 12.6 $\mathrm{kJ} / \mathrm{mol}[42]$.

The external (solute-solvent) potsntial can be divided irito isotropic and aniso- 
tropic components :

$$
U_{\text {ext }}(n, \Omega)=U_{\text {ext }}^{\text {iso }}(n)+U_{\text {ext }}^{\text {aniso }}(n, \Omega)
$$

The isotropic, conformation-dependent, component will be modeled as a correction to the gas-phase trans-gauche energy difference, $E_{\mathrm{g}}$ in Equation 3.11, due to "solvent pressure" :

$$
U_{\text {ext }}^{\text {iso }}(n)=n_{\mathrm{g}} E_{\mathbf{g}}^{\text {iso }}
$$

though other parameterizations of this "solvent pressure" are possible. This portion of the solute-solvent potential can be combined with the internal part of the potential in Equation 3.11 to give :

$$
U^{\text {iso }}(n)=n_{\mathrm{g}} E_{\mathrm{g}}^{\text {eff }}+n_{\mathrm{g}+\mathrm{g}}-E_{\mathrm{g}+\mathrm{g}}
$$

using an effective trans-gauche energy difference, $E_{\mathrm{g}}^{\text {eff }}=E_{\mathrm{g}}+E_{\mathrm{g}}^{\text {iso }}$. The value of $E_{\mathrm{g}}^{\text {eff }}$ depends not only on the internal coordinates of the solute molecule, but also on the isotropic solute-solvent interaction and determines the conformational distribution observed in solution. $E_{\mathrm{g}}^{\text {eff }}$ has been measured by different methods to be $2.1-2.5$ $\mathrm{kJ} / \mathrm{mol}$ [43] and will be used as an adjustable parameter in modeling the alkanes. The total potential can now be written :

$$
U(n, \Omega)=U^{\text {iso }}(n)+U_{\text {ext }}^{\text {aniso }}(n, \Omega)
$$

where $U^{\text {iso }}(n)$ is defined by Equation 3.14 and $U_{\text {ext }}^{\text {aniso }}(n, \Omega)$ will bf defined by a mean field model for the anisotropic solute-solvent interaction. The conformer probabilities, $p^{n}$, and order tensors, $S_{\alpha, \beta}^{n}$, can be expressed in terms of the potential $U(n, \Omega) \cdot p^{\tilde{n}}$ can be written in terms of $U(n, \Omega)$ as :

$$
p^{n}=G(n) \frac{\exp \left(-\frac{U^{\mathrm{ieo}}(n)}{k T}\right) Z_{\text {ext }}^{n}}{Z_{\text {tot }}}
$$


where $G(n)=\left(I_{x} I_{y} I_{z}\right)^{\frac{1}{2}}$ is the confomer rotational kinetic energy factor [1] (which has a very small effect on the calculated probabilities) and

$$
Z_{\text {ext }}^{n}=\int \exp \left(-\frac{U_{\text {ext }}^{\text {aniso }}(n, \Omega)}{k T}\right) d \Omega
$$

and

$$
Z_{\mathrm{tot}}=\sum_{n} G(n) \exp \left(-\frac{U^{\mathrm{iso}}(n)}{k T}\right) Z_{\mathrm{ext}}^{n}
$$

are the external and total partition functions. The average in Equation 3.8 can be written in terms of $U_{\text {ext }}^{\text {aniso }}(n, \Omega)$ :

$$
S_{\alpha, \beta}^{n}=\frac{1}{2 Z_{\text {ext }}^{n}} \int\left(3 \cos \theta_{\alpha} \cos \theta_{\beta}-\delta_{\alpha \beta}\right) \exp \left(-\frac{U_{\text {ext }}^{\text {aniso }}(n, \Omega)}{k T}\right) d \Omega .
$$

For the modeling in the next few sections, $G(n)$ will be used with Model $\mathrm{D}$ but will not be used in the other models for consistency with previous uses of the models and because of its negligible effect on the results of the modeling.

\subsection{Models for $U_{\text {ext }}^{\text {aniso }}(n, \Omega)$}

Several mean-field models for the external potential $U_{\text {ext }}^{\text {aniso }}(n, \Omega)$ describing the solute-solvent interaction were examined and used to simulate the measured dipole couplings of the $n$-alkanes. The first, Model A, is a phenonıenological model that was introduced by van der Est, et al. [44] and describes the liquid crystal solvent as an elastic continuum distorted by the presence of the solute molecule. This potential is expressed in terms of the circumference of the projection of the solute molecule into a plane perpendicular to the nematic director :

$$
U_{\text {ext }}^{\text {aniso }}\left(r_{l}, \Omega\right)=k c^{2}(n, \Omega)
$$


where $k$ is an adjustable constant describing the strength of the interaction between the solute molecule and the solvent mean-field, and $c$ is the minimum circumference [44] around the projection of the solute molecule, pictured in Figure 3.11. This model has also been extended by Zimmerman, et al. [45] to include a term which accounts for the length of the elastic tube along the director of the liquid crystal. The potential then becomes :

$$
U_{\mathrm{ext}}^{\text {aniso }}(n, \Omega)=k c^{2}(n, \Omega)-\zeta z(\Omega) c(\Omega)
$$

where $k$ and $\zeta$ are adjustable constants describing the strength of the solute-solvent interaction, $c(n, \Omega)$ has the same meaning as in Equation 3.20, and $z(n, \Omega)$ is the length of the projection of the solute molecule along the liquid crystal director, as shown in Figure 3.11.

The other models used in this study are based on a potential of mean torque for a solute molecule in a uniaxial mean field. The total external potential (including the isotropic portion), truncated to second rank, can be expressed $[20,46,47]$ :

$$
U_{\text {ext }}(n, \Omega)=u_{0,0}^{n} D_{0,0}^{0}(\Omega)+\sum_{m} u_{2, m}^{n} D_{m, 0}^{2}(\Omega)
$$

where the $u_{l, m}^{n}$ are coefficients describing the solute-solvent interaction and the $D_{m, m^{\prime}}^{l}$ are Wigner rotation matrices. This form for the potential can be derived by considering long-range [46] or short-range [20] forces as the primary intermolecular interactions in the liquid crystal or simply by expanding an unknown interaction in an infinite series of spherical harmonics [47], yet all lead to the same form for the potential. Thus it is not clear what type(s) of forces may be responsible for ordering in liquid crystals, so three parameterizations of the $u_{2, m}^{n}$ coefficients will be used in this thesis. The zero-rank term in Equation 3.22 is the same as the isotropic, 


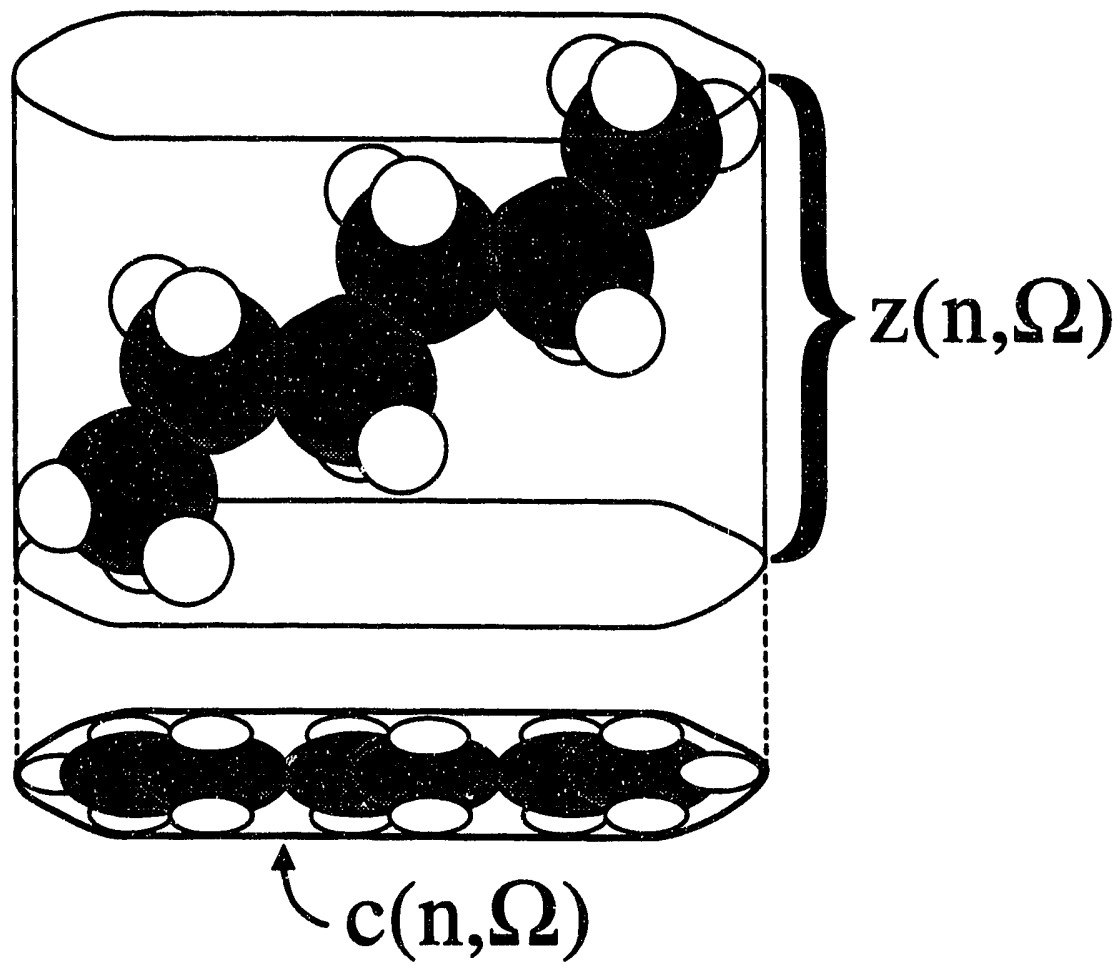

Figure 3.11: Model A. $z(n, \Omega)$ is the length of the projection of conformer $n$ along the director of the liquid crystal as a function of orientation of the molecule, $\Omega . c(n, \Omega)$ is the minimum circumference around the projection of the van der Waals radii of the atoms of conformer $n$ into a plane perpendicular to the director of the liquid crystal. 
conformation-dependent solute-solvent interaction, $U_{\text {ext }}^{\text {iso }}(n)$ in Equation 3.12, and will be modeled as in Equation 3.13. The remaining second-rank terms comprise the anisotropic portion of the solute-solvent interaction :

$$
U_{\text {ext }}^{\text {aniso }}(n, \Omega)=\sum_{m} u_{2, m}^{n} D_{m, 0}^{2}(\Omega)
$$

$u_{2, m}^{n}$ is a spherical tensor representation of the interaction between a particular conformation, $n$, of the solute molecule and the solvent mean field. This tensor can be written in its principal axis system leaving only $u_{2,0}^{n}$ and $u_{2, \pm 2}^{n}$ non-zero. Then Equation 3.23 can be written :

$$
U_{\text {ext }}^{\text {aniso }}(n, \Omega)=u_{2,0}^{n} d_{0,0}^{2}(\beta)+2 u_{2,2}^{n} d_{2,0}^{2}(\beta) \cos (2 \alpha)
$$

where $d_{0,0}^{2}=\frac{1}{2}\left(3 \cos ^{2} \beta-1\right)$ and $d_{2,0}^{2}=\sqrt{\frac{3}{8}} \sin ^{2} \beta$ are reduced Wigner rotation matrices describing the orientation of the molecule's PAS with respect to the nematic director.

The first mean-torque potential (Model B) parameterizes the $u_{2, m}^{n}$ coefficients in Equation 3.24 using a model for the solute-solvent interaction in which the interacting molecules are represented by parallelepipeds. This interaction is not a true tensor. However, it appears to be successful in modeling solute-liquid crystal interactions $[40,48]$ and will be used here. The coefficients $u_{2, m}^{n}$ are written in terms of the length (L), width (W), and breadth (B) of the parallelepiped representing the solute molecule $[40,48]$ :

$$
\begin{aligned}
& u_{2,0}^{n}=\frac{\epsilon}{3}\left[6 L B W+L\left(W^{2}+B^{2}\right)-2 W\left(L^{2}+B^{2}\right)-2 B\left(W^{2}+L^{2}\right)\right] \\
& u_{2,2}^{n}=\frac{\epsilon}{\sqrt{6}}\left[\left(L^{2}-B W\right)(B-W)\right] .
\end{aligned}
$$

The length, width, and breadth of each conformer of the molecule are calculated from the semiaxes of the ellipsoid of inertia of the conformer [40] so that $L, B$, and 
$W$ are coincident with the principal axes of the inertia tensor of the solute molecule, pictured in Figure 3.12. L, B, and W are calculated :

$$
\begin{aligned}
L & =\sqrt{\frac{5}{2 m}\left(I_{x x}+I_{y y}-I_{z z}\right)} \\
B & =\sqrt{\frac{5}{2 m}\left(I_{x x}+I_{z z}-I_{y y}\right)} \\
W & =\sqrt{\frac{5}{2 m}\left(I_{y y}+I_{z z}-I_{x x}\right)}
\end{aligned}
$$

where $m$ is the mass of the solute molecule and $I_{\alpha \alpha}$ are the diagonal elements of particular conformation's Cartesian inertia tensor. $\epsilon$ in Equation 3.25 is an adjustable parameter describing the strength of the solute-solvent interaction.

The second mean-field model (Model C) uses the inertia tensor for the $u_{2, m}^{n}$ coefficients. The spherical tensor coefficients are calculated from the Cartesian inertia tensor :

$$
\begin{aligned}
& u_{x x}=\sum_{i} m_{i}\left(y_{i}^{2}+z_{i}^{2}\right) \\
& u_{y y}=\sum_{i} m_{i}\left(x_{i}^{2}+z_{i}^{2}\right) \\
& u_{z z}=\sum_{i} m_{i}\left(x_{i}^{2}+y_{i}^{2}\right) \\
& u_{x y}=-\sum_{i} m_{i} x_{i} y_{i} \\
& u_{x z}=-\sum_{i} m_{i} x_{i} z_{i} \\
& u_{y z}=-\sum_{i} m_{i} y_{i} z_{i}
\end{aligned}
$$

where $x_{i}, y_{i}$, and $z_{i}$ are the distances of atom $i$ from the center of mass of a given conformation and $m$ is the mass of the atom. The spherical tensor elements $u_{2, m}^{n}$ are constructed from the diagonalized Cartesian tensor elements as in Table 1.2. This model can be pictured as the liquid crystal solvent orienting an anisotropicallyshaped molecule, where the shape anisotropy is quantified by the inertia tensor. 


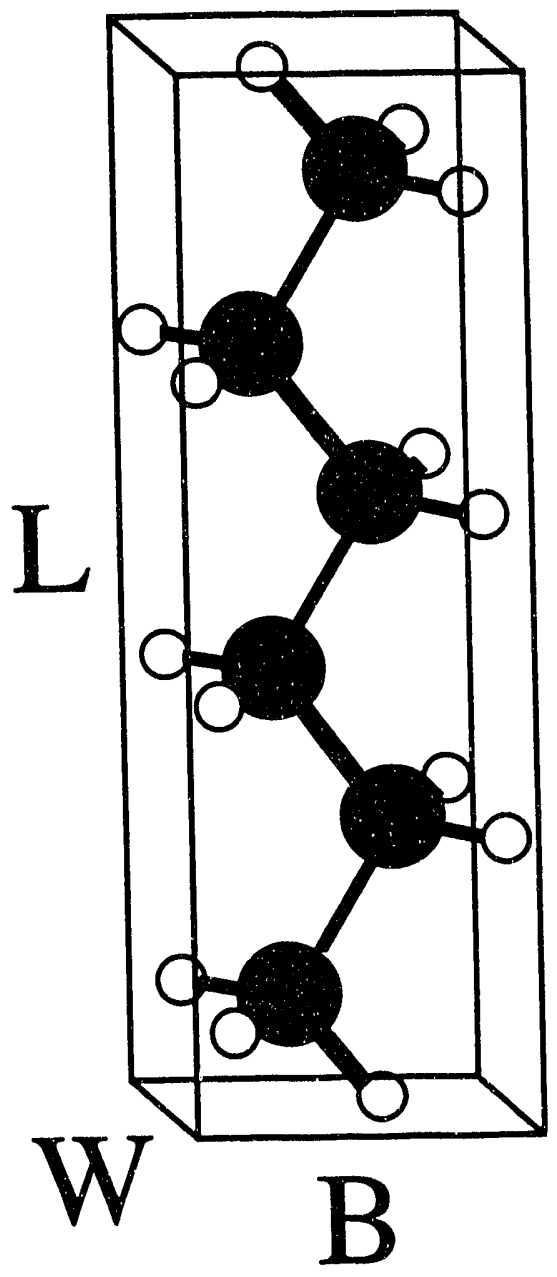

Figure 3.12: Model B. L, B, and $\mathrm{W}$ are the dimensions of a parallelipiped representing each alkane conformer. $\mathrm{L}, \mathrm{B}$, and $\mathrm{W}$ are coincident with the principal axes of the inertia tensor. 
The other mean-torque potential (Model D) [1] parameterizes the $u_{2, m}^{n}$ as an interaction between individual carbon-carbon bonds and the nematic mean field, and as an interaction between pairs of adjacent carbon-carbon bonds and the nematic mean field. The $u_{2, m}^{n}$ are derived from the Cartesian tensor [1, 49] :

$$
u_{a b}=-\frac{2}{3} \tilde{w}_{0} \sum_{i=1}^{N}\left[3 s_{a}^{i} s_{b}^{i}-\delta_{a b} \mathbf{s}^{i} \cdot \mathbf{s}^{i}\right]-\frac{2}{3} \tilde{w}_{1} \sum_{i}^{N-1}\left[3\left(\frac{s_{a}^{i} s_{b}^{i+1}+s_{a}^{i+1} s_{b}^{i}}{2}\right)-\delta_{a b} \mathrm{~s}^{i} \cdot \mathrm{s}^{i+1}\right]
$$

where $\mathbf{s}^{i}$ is a unit vector in the direction of carbon-carbon bond $i, s_{\alpha}^{i}$ is the $\alpha(=$ $x, y, z)$ component of that unit vector, and $\tilde{w}_{0}$ and $\tilde{w}_{1}$ are adjustable constants describing the strength of the solute-liquid crystal interaction. The first sum in Equation 3.28 accounts for the interaction aligning individual carbon-carbon bonds, and the second sum describes the orientation of adjacent pairs of carbon-carbon bonds by the nematic mean-field. As discussed by Photinos, et al. [1, 49], this equation can be rewritten in terms of chords connecting the midpoints of adjacent carbon-carbon bonds. In this equivalent representation, ordering of the $n$-alkane is due to alignment of individual carbon-carbon bonds and alignment of chords connecting adjacent carbon-carbon bonds, as pictured in Figure 3.13.

The $u_{2, m}^{n}$ can be constructed frorn the diagonalized Cartesian tensor of Equation 3.28 :

$$
\begin{aligned}
& u_{2,0}^{n}=\frac{1}{\sqrt{6}}\left[2 u_{33}-\left(u_{11}+u_{22}\right)\right] \\
& u_{2,2}^{n}=\frac{1}{2}\left(u_{11}-u_{22}\right)
\end{aligned}
$$

where $u_{11}, u_{22}$, and $u_{33}$ are the principal values of the Cartesian tensor of Equation 3.28. This potential will be tested with one adjustable parameter, $\tilde{w}_{0}=\check{w}_{1}$, and with two adjustable parameters, $\tilde{w}_{0}$ and $\tilde{w}_{1}$.

To test the mean field models, the experimental dipole couplings will be simu- 

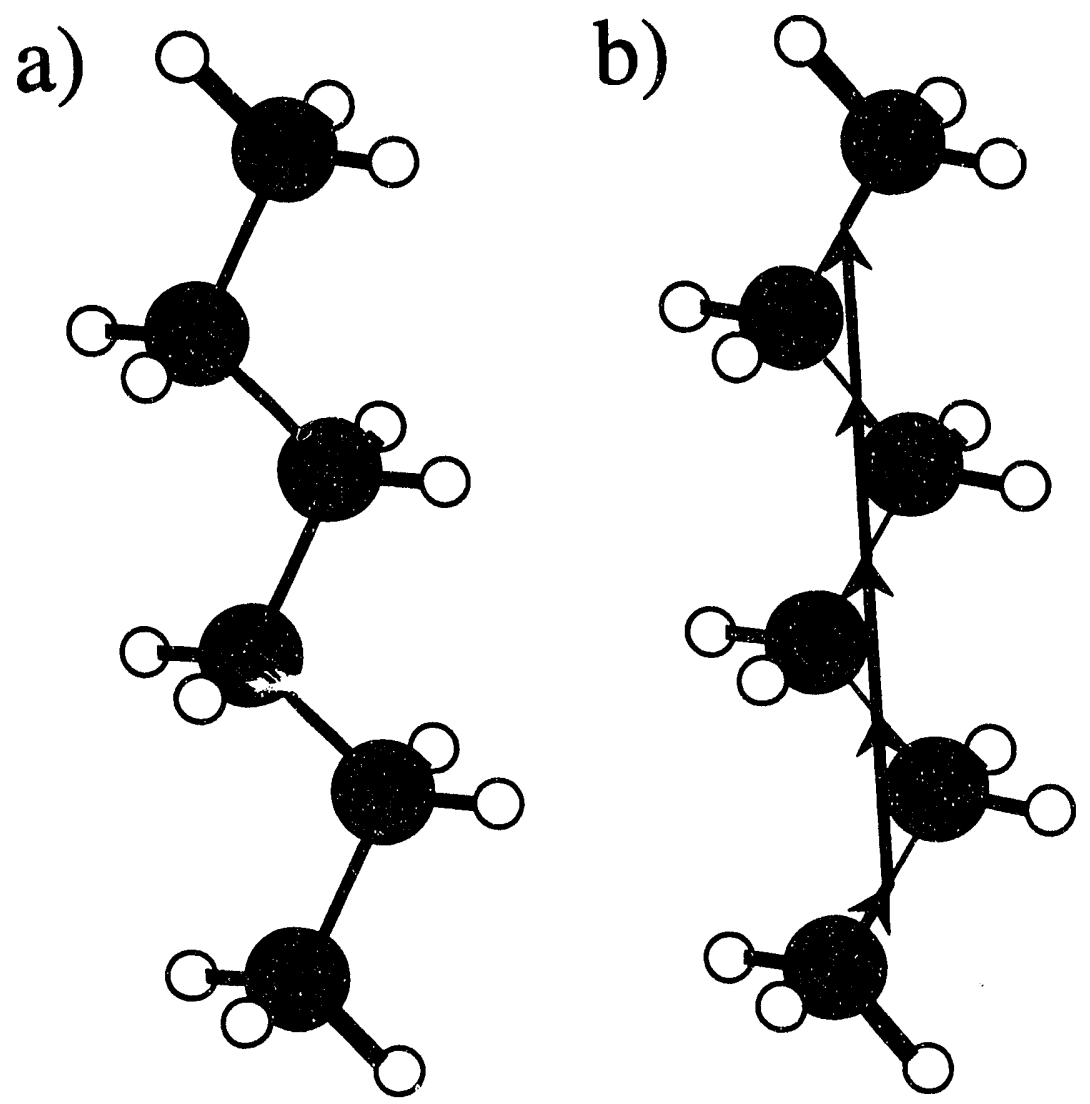

Figure 3.13: Model D. a) The single carbon-carbon bond self-correleation terms of the tensor in Equation 3.28 can be interpreted as alignment of each carbon-carbon bond by the nematic mean field. 'The importance of the single bond terms is determined by the value of the multiplier of this term, $\left(\tilde{w}_{1}-\tilde{w}_{0}\right)$ [1]. b) The second sum in Equation 3.28 can be rewritten in terms of chords connecting the midpoints of adjacent carbon-carbon bonds [1]. This term can be interpreted as the nematic mean field aligning the chords and the terminal carbon-carbon bonds. The importance of this term is determined by its coefficient, $\tilde{w}_{1}$. 
lated using the RIS model for the isotropic part of the alkane solute potential and one of the four mean-field models just described for the anisotropic part. The simulations will be done using either two or three adjustable parameters : one being the effective trans-gauche energy difference, $E_{\mathbf{g}}^{\text {eff }}$, and the other one or two describing the strength of the solute-solvent interaction.

\subsection{Results of Two-Adjustable-Parameter Mod- eling}

Simulations of the experimental dipole couplings of the alkanes were performed with the two- and three-parameter versions of the models described in the previous sections. The adjustable parameters were varied using a simplex minimization routine to optimize the root-mean-squared (RMS) difference between the calculated and experimental couplings. All minimizations were performed with the alkane geometrical parameters from Table 3.5.

Table 3.5: Geometrical parameters of alkanes.

\begin{tabular}{|l|c|}
\hline Length of C-H bond & $1.09 \AA$ \\
Length of C-C bond & $1.53 \AA$ \\
Bond Angle HCH of methylene group & $109.0^{\circ}$ \\
Bond Angle HCH of methyl group & $109.47^{\circ}$ \\
Bond Angle CCC & $112^{\circ}$ \\
Dihedral Angle for gauche conformations & $\pm 112.5^{\circ}$ \\
van der Waals radius of H & $1.2 \AA$ \\
van der Waals radius of C & $1.7 \AA$ \\
Mass of H & 2.0 \\
Mass of C & 12.0 \\
\hline
\end{tabular}

The two-parameter fits used the strength of the solute-solvent interaction $(k, \epsilon$, 
or $\left.\tilde{w}_{0}=\tilde{w}_{1}\right)$ and the effective trans-gauche energy difference $\left(E_{\mathrm{g}}^{\text {eff }}\right)$ as adjustable parameters. The RMS deviation between the calculated and experimental dipole couplings in Table 3.6 and the calculated dipole couplings in Tables 3.7-3.11 show that all models fit the couplings of the shorter alkanes better than the longer, probably due in part to the greater number of couplings on the longer alkanes. Model B fit the short alkanes the best and Model D fit the long alkanes best. The adjustable parameters show a variation with molecular size - the solute-solvent interaction strength parameters $\epsilon, k$, and $\tilde{w}_{0}=\tilde{w}_{1}$, all decrease in magnitude with increasing chain length. The other adjustable parameter, $E_{\mathrm{g}}^{\text {eff }}$, counters this by increasing with increasing chain length. Both of these effects are much more pronounced in Models A, B, and C than in Model D. In fact, the values of $E_{\mathrm{g}}^{\text {eff }}$ in Models A, B, and $\mathrm{C}$ grow larger for decane than the gas phase value of $E_{\mathrm{g}}$ found for butane of $3.8 \mathrm{~kJ} / \mathrm{mol}$ [43]. Comparison with experimentally determined values of $E_{\mathrm{g}}^{\text {eff }}$ found for butane in the liquid state ( $E_{\mathrm{g}}^{\text {eff }}$ approximately $2.1-2.5 \mathrm{~kJ} / \mathrm{mol}[43]$ ) suggests that such large values of $E_{\mathbf{g}}^{\text {eff }}$ are physically unreasonable. While it is possible that the orienting effect of the liquid crystal might skew the conformer population distribution to favor elongated conformers, resulting in a higher $E_{\mathrm{g}}^{\text {eff }}$, it should nat be so pronounced in a "liquid-like" system such as this. Models A, B, and C appear to work well only for small molecules, while Model D works for molecules of all sizes and has values of adjustable parameters which remain relatively constant as the size of the alkane changes, allowing prediction of dipole couplings for longer alkanes by extrapolation from the values of adjustable parameters found for these alkanes. Thus it appears that Model D, which is an additive potential constructed from interactions of molecular subunits with ihe liquid crystal mean field outper- 
forms Models A, B, and C which model the overall shape of the solute molecule interacting with the liquid crystal mean field.

Table 3.6: Results of two-adjustable-parameter fits to experimental dipole couplings.

\begin{tabular}{|c|c|c|c|c|c|c|}
\hline & & Hexane & Heptane & Octane & Nonane & Decane \\
\hline \multirow{3}{*}{ Model A } & $\begin{array}{c}k \\
\left(10^{-3} \mathrm{Nm}^{-1}\right)\end{array}$ & 3.97 & 3.30 & 2.88 & 2.44 & 2.15 \\
\hline & $\begin{array}{c}E_{\mathbf{g}}^{\text {eff }} \\
\left(k J m o m^{-1}\right)\end{array}$ & 3.27 & 3.45 & 3.72 & 3.89 & 4.22 \\
\hline & $\begin{array}{l}\text { RMS Dev. } \\
(\mathrm{Hz})\end{array}$ & 88 & 109 & 122 & 151 & 153 \\
\hline \multirow{3}{*}{ Model B } & $\begin{array}{c}\epsilon \\
\left(10^{4} \mathrm{kJmol}^{-1} \mathrm{~m}^{-3}\right)\end{array}$ & 2.13 & 1.57 & 1.21 & 0.92 & 0.74 \\
\hline & $\begin{array}{c}E_{\mathbf{g}}^{\text {eff }} \\
\left(k J \mathrm{~mol}^{-1}\right)\end{array}$ & 3.07 & 3.35 & 3.75 & 4.03 & 4.47 \\
\hline & $\begin{array}{l}\text { RMS Dev. } \\
(\mathrm{Hz})\end{array}$ & 35 & 54 & 83 & 105 & 112 \\
\hline \multirow{3}{*}{ Model C } & $\begin{array}{c}\epsilon \\
\left(J k g^{-1} m^{-2} m^{-1}\right)\end{array}$ & 1.45 & 1.01 & 0.74 & 0.54 & 0.42 \\
\hline & $\begin{array}{c}E_{\mathbf{g}}^{\text {eff }} \\
\left(k J \mathrm{~mol}^{-1}\right)\end{array}$ & 2.96 & 3.16 & 3.56 & 3.72 & 4.11 \\
\hline & $\begin{array}{c}\text { RMS Dev. } \\
(\mathrm{Hz})\end{array}$ & 93 & 120 & 142 & 175 & 178 \\
\hline \multirow{3}{*}{ Model D } & $\begin{array}{c}\tilde{w}_{0}=\tilde{w}_{1} \\
\left(k J m^{-1}\right)\end{array}$ & 0.806 & 0.765 & 0.754 & 0.738 & 0.714 \\
\hline & $\begin{array}{c}E_{\mathrm{g}}^{\mathrm{eff}} \\
\left(k J \mathrm{~mol}^{-1}\right) \\
\end{array}$ & 2.16 & 2.28 & 2.35 & 2.28 & 2.51 \\
\hline & $\begin{array}{l}\text { RMS Dev. } \\
(\mathrm{Hz})\end{array}$ & 74 & 77 & 79 & 93 & 90 \\
\hline
\end{tabular}

Having fit the dipole couplings, the models allows us to calculate order parameters and conformational probabilities for the alkanes. Table 3.12 shows the order parameters calculated using the best-fit parameters in Table 3.6. It can be seen that all four models give similar results for the values of the order parameters.

Table 3.13 lists the probabilities of having different numbers of gauche bonds 
Table 3.7: Dipole couplings for hexane from the two-adjustable-parameter fits to the experimental couplings.

\begin{tabular}{|c||r|r|r|r|r|}
\hline \multicolumn{1}{|c||}{} & \multicolumn{6}{c|}{ Dipole Couplings (Hz) } \\
\hline Protons & Experiment & Model A & Model B & Model C & ModeI D \\
\hline \hline $1-2$ & 1526 & 1493 & 1455 & 1482 & 1573 \\
$4-5$ & 3288 & 3161 & 3280 & 3149 & 3171 \\
$6-7$ & 3711 & 3702 & 3737 & 3709 & 3686 \\
$1-4$ & -310 & -262 & -293 & -257 & -305 \\
$1-6$ & -852 & -919 & -836 & -919 & -901 \\
$1-8$ & -484 & -576 & -501 & -568 & -585 \\
$1-10$ & -272 & -276 & -242 & -275 & -273 \\
$1-12$ & -167 & -176 & -154 & -176 & -172 \\
$4-8$ & -1323 & -1516 & -1380 & -1532 & -1440 \\
$4-9$ & -881 & -963 & -850 & -967 & -990 \\
$4-6$ & - & 92 & 2 & 100 & 134 \\
$4-7$ & - & 156 & 141 & 161 & 182 \\
$4-10$ & - & -761 & -666 & -760 & -767 \\
$4-11$ & - & -561 & -470 & -554 & -580 \\
$6-8$ & - & 21 & -91 & 17 & -40 \\
$6-9$ & - & 121 & 96 & 124 & 74 \\
\hline
\end{tabular}

in the chain for the different models. Alongside each probability is the probability of the conformers in an isotropic liquid with the same values of $E_{\mathrm{g}}^{\text {eff }}$ (that is, with $\epsilon, k$, or $\tilde{w}_{0}=\tilde{w}_{1}$ set to 0 ). The total number of conformers for each molecule is listed, along with the number of conformers with a given number of gauche bonds, excluding conformers with adjacent gauche ${ }^{+}$and gauche $^{-}$bonds, which have nearly zero probability. From this table it can be seen that the anisotropic solute-solvent interaction has little effect on the conformer distribution - the value of $E_{\mathbf{g}}^{\text {eff }}$ is primarily what determines the conformer distribution. This confirms the notion that the alkanes in a "liquid-like" liquid crystal such as this one behave very similarly to the neat liquid alkane, and also suggests that Models A, B, and C, with their high $E_{\mathrm{g}}^{\text {eff }}$, do not provide good models for solute-solvent interaction. It can also be seen from Model D that the small effect the anisotropic solute-solvent potential does have on the conformer distribution is to slightly favor elongated 
Table 3.8: Dipole couplings for heptane from the two-adjustable-parameter fits to the experimental couplings.

\begin{tabular}{|c||r|r|r|r|r|}
\hline \multicolumn{1}{|c||}{} & \multicolumn{5}{|c|}{ Dipole Couplings (Hz) } \\
\hline Protons & Experiment & Model A & Model B & Model C & Mode D \\
\hline \hline $1-2$ & 1489 & 1421 & 1417 & 1412 & 1495 \\
$4-5$ & 3461 & 3211 & 3351 & 3182 & 3305 \\
$6-7$ & 3897 & 3860 & 3898 & 3859 & 3845 \\
$8-9$ & 4093 & 4122 & 4172 & 4127 & 4098 \\
$1-4$ & -226 & -215 & -259 & -218 & -248 \\
$1-6$ & -916 & -945 & -875 & -939 & -947 \\
$1-8$ & -520 & -572 & -509 & -568 & -605 \\
$1-10$ & -290 & -293 & -262 & -292 & -299 \\
$1-12$ & -192 & -199 & -176 & -199 & -202 \\
$1-14$ & -127 & -127 & -112 & -127 & -133 \\
$4-8$ & -1453 & -1646 & -1524 & -1658 & -1551 \\
$4-9$ & -924 & -994 & -890 & -994 & -1029 \\
$6-10$ & -1575 & -1792 & -1652 & -1820 & -1700 \\
$6-11$ & -975 & -1044 & -938 & -1053 & -1073 \\
$4-6$ & - & 67 & -9 & 79 & 65 \\
$4-7$ & - & 141 & 131 & 149 & 139 \\
$4-10$ & - & -817 & -726 & -814 & -851 \\
$4-11$ & - & -575 & -492 & -567 & -609 \\
$4-12$ & - & -322 & -288 & -324 & -323 \\
$4-13$ & - & -315 & -282 & -317 & -320 \\
$6-8$ & - & 106 & -8 & 101 & 90 \\
$6-9$ & - & 177 & 153 & 181 & 163 \\
\hline
\end{tabular}


Table 3.9: Dipole couplings for octane from the two-adjustable-parameter fits to the experimental couplings.

\begin{tabular}{|c||r|r|r|r|r|}
\hline \multicolumn{1}{|c||}{} & \multicolumn{5}{c|}{ Dipole Couplings (Hz) } \\
\hline Protons & Experiment & Model A & Model B & Model C & ModeI D \\
\hline \hline $1-2$ & 1637 & 1516 & 1495 & 1494 & 1626 \\
$4-5$ & 3539 & 3298 & 3424 & 3259 & 3356 \\
$6-7$ & 4119 & 4018 & 4079 & 4012 & 4058 \\
$8-9$ & 4319 & 4389 & 4432 & 4398 & 4358 \\
$1-4$ & -319 & -255 & -282 & -247 & -297 \\
$1-6$ & -949 & -986 & -913 & -970 & -974 \\
$1-8$ & -538 & -563 & -504 & -551 & -625 \\
$1-10$ & -296 & -300 & -269 & -297 & -311 \\
$1-12$ & -204 & -208 & -184 & -204 & -218 \\
$1-14$ & -129 & -140 & -122 & -137 & -150 \\
$1-16$ & -96 & -95 & -83 & -93 & -103 \\
$4-8$ & -1544 & -1780 & -1669 & -1805 & -1653 \\
$4-9$ & -976 & -1014 & -912 & -1006 & -1082 \\
$6-10$ & -1710 & -1979 & -1855 & -2041 & -1851 \\
$6-11$ & -1088 & -1099 & -984 & -1094 & -1136 \\
$4-6$ & - & 130 & 45 & 138 & 154 \\
$4-7$ & - & 182 & 168 & 189 & 201 \\
$4-10$ & - & -838 & -739 & -819 & -905 \\
$4-11$ & - & -564 & -479 & -541 & -630 \\
$4-12$ & - & -355 & -316 & -353 & -360 \\
$4-13$ & - & -344 & -305 & -339 & -355 \\
$4-14$ & - & -223 & -201 & -224 & -226 \\
$4-15$ & - & -228 & -199 & -223 & -244 \\
$6-8$ & - & 100 & -16 & 95 & 15 \\
$6-9$ & - & 189 & 157 & 187 & 124 \\
$6-12$ & - & -902 & -787 & -868 & -960 \\
$6-13$ & - & -602 & -505 & -572 & -654 \\
$8-10$ & - & 160 & 68 & 188 & 225 \\
$8-11$ & - & 228 & 212 & 244 & 256 \\
\hline
\end{tabular}


Table 3.10: Dipole couplings for nonane from the two-adjustable-parameter fits to the experimental couplings.

\begin{tabular}{|c||r|r|r|r|r|}
\hline \multicolumn{1}{|c||}{} & \multicolumn{5}{c|}{ Dipole Couplings (Hz) } \\
\hline Protons & Experiment & Model A & Model B & Model C & Model D \\
\hline \hline $1-2$ & 1546 & 1405 & 1431 & 1392 & 1514 \\
$4-5$ & 3570 & 3214 & 3353 & 3151 & 3326 \\
$6-7$ & 4104 & 3920 & 3991 & 3897 & 3987 \\
$8-9$ & 4420 & 4387 & 4450 & 4386 & 4417 \\
$10-11$ & 4420 & 4525 & 4537 & 4533 & 4475 \\
$1-4$ & -265 & -215 & -260 & -216 & -246 \\
$1-6$ & -958 & -960 & -903 & -940 & -967 \\
$1-8$ & -539 & -528 & -473 & -520 & -629 \\
$1-10$ & -300 & -291 & -264 & -288 & -317 \\
$1-12$ & -206 & -201 & -178 & -199 & -222 \\
$1-14$ & -138 & -139 & -122 & -136 & -157 \\
$1-16$ & -93 & -100 & -87 & -98 & -113 \\
$1-18$ & -73 & -71 & -61 & -69 & -82 \\
$4-8$ & -1554 & -1788 & -1695 & -1793 & -1638 \\
$4-9$ & -977 & -977 & -878 & -964 & -1071 \\
$6-10$ & -1721 & -2021 & -1908 & -2070 & -1866 \\
$6-11$ & -1028 & -1082 & -975 & -1078 & -1160 \\
$8-12$ & -1779 & -2076 & -1970 & -2151 & -1909 \\
$8-13$ & -1097 & -1120 & -1001 & -1114 & -1176 \\
$4-6$ & - & 97 & 37 & 113 & 90 \\
$4-7$ & - & 164 & 160 & 171 & 158 \\
$4-10$ & - & -807 & -714 & -791 & -916 \\
$4-11$ & - & -531 & -455 & -514 & -645 \\
$4-12$ & - & -355 & -318 & -353 & -367 \\
$4-13$ & - & -340 & -303 & -336 & -361 \\
$4-14$ & - & -228 & -203 & -228 & -238 \\
$4-15$ & - & -231 & -201 & -225 & -259 \\
$4-16$ & - & -152 & -132 & -149 & -168 \\
$4-17$ & -151 & -131 & -148 & -167 \\
$6-8$ & - & 156 & 30 & 142 & 105 \\
$6-9$ & - & 217 & 183 & 213 & 179 \\
$6-12$ & -894 & -778 & -868 & -1006 \\
$6-13$ & - & -577 & -485 & -554 & -673 \\
$6-14$ & -372 & -333 & -372 & -388 \\
$6-15$ & -358 & -317 & -353 & -382 \\
$8-10$ & 129 & 52 & 164 & 140 \\
$8-11$ & 219 & 205 & 234 & 206 \\
\hline & & & & &
\end{tabular}


Table 3.11: Dipole couplings for decane from the two-adjustable-parameter fits to the experimental couplings.

\begin{tabular}{|c|c|c|c|c|c|}
\hline & \multicolumn{5}{|c|}{ Dipole Couplings $(\mathrm{Hz})$} \\
\hline Protons & Experiment & Model A & Model B & Model C & Model D \\
\hline$\overline{1-2}$ & 1669 & $\overline{1538}$ & $\overline{1524}$ & 1497 & 1639 \\
\hline $4-5$ & 3660 & 3334 & 3482 & 3279 & 3411 \\
\hline $6-7$ & 4283 & 4040 & 4134 & 4025 & 4149 \\
\hline $8-9$ & 4577 & 4544 & 4602 & 4537 & 4574 \\
\hline $10-11$ & 4697 & 4807 & 4822 & 4808 & 4787 \\
\hline $1-4$ & -313 & -270 & -290 & -252 & -294 \\
\hline $1-6$ & -998 & -1001 & -945 & -982 & -996 \\
\hline $1-8$ & -556 & -513 & -458 & -502 & -623 \\
\hline $1-10$ & -319 & -292 & -265 & -289 & -319 \\
\hline $1-12$ & -219 & -199 & -176 & -195 & -225 \\
\hline $1-14$ & -151 & -139 & -121 & -135 & -158 \\
\hline $1-16$ & -127 & -102 & -88 & -98 & -117 \\
\hline $1-18$ & -82 & -75 & -64 & -73 & -87 \\
\hline $1-20$ & -57 & -55 & -47 & -53 & -64 \\
\hline $4-8$ & -1666 & -1915 & -1827 & -1925 & -1745 \\
\hline $4-9$ & -1020 & -973 & -878 & -964 & -1094 \\
\hline $6-10$ & -1863 & -2179 & -2060 & -2233 & -2003 \\
\hline $6-11$ & -1062 & -1091 & -978 & -1082 & -1177 \\
\hline 8-12 & -1970 & -2289 & -2164 & -2374 & -2085 \\
\hline $8-13$ & -1140 & -1160 & -1035 & -1151 & -1235 \\
\hline 46 & - & 165 & 72 & 158 & 165 \\
\hline $4-7$ & - & 203 & 190 & 205 & 208 \\
\hline $4-10$ & - & -788 & -694 & -770 & -925 \\
\hline $4-11$ & - & -496 & -425 & -479 & -624 \\
\hline $4-12$ & - & -367 & -330 & -365 & -382 \\
\hline $4-13$ & - & -345 & -309 & -341 & -373 \\
\hline $4-14$ & - & -231 & -204 & -229 & -248 \\
\hline $4-15$ & - & -230 & -199 & -223 & -263 \\
\hline $4-16$ & - & -158 & -136 & -153 & -175 \\
\hline $4-17$ & - & -156 & -135 & -151 & -174 \\
\hline $4-18$ & - & -109 & -94 & -106 & -124 \\
\hline 4-19 & - & -109 & -94 & -106 & -124 \\
\hline $6-8$ & - & 133 & 23 & 134 & 48 \\
\hline $6-9$ & - & 217 & 191 & 218 & 150 \\
\hline $6-12$ & - & -891 & -774 & -856 & -1016 \\
\hline $6-13$ & - & -562 & -471 & -532 & -672 \\
\hline $6-14$ & - & -392 & -350 & -392 & -406 \\
\hline $6-15$ & - & -371 & -328 & -365 & -395 \\
\hline $6-16$ & - & -243 & -214 & -239 & -257 \\
\hline $6-17$ & - & -241 & -207 & -232 & -273 \\
\hline $8-10$ & - & 206 & 99 & 225 & 248 \\
\hline $8-11$ & - & 268 & 245 & 280 & 278 \\
\hline $8-14$ & - & -920 & -790 & -884 & -1055 \\
\hline $8-15$ & - & -576 & -475 & -542 & -676 \\
\hline
\end{tabular}


conformers (e.g. all-trans, and conformers with only one gauche bond) in relation to the isotropic conformer distribution. The other notable feature is that, especially for longer alkanes, a significant number of the possible conformers have adjacent gauche $^{+}$and gauche ${ }^{-}$bonds and almost never occur in solution.

Table 3.12: Order parameters from two-adjustable-parameter fits to experimental dipole couplings.

\begin{tabular}{|l||r|r|r|r|}
\hline \multicolumn{1}{|c||}{} & \multicolumn{4}{c|}{$S_{z z}$} \\
\cline { 2 - 5 } & Model A & Model B & Model C & Model D \\
\hline \hline Hexane & 0.19 & 0.17 & 0.19 & 0.19 \\
Heptane & 0.21 & 0.19 & 0.21 & 0.22 \\
Octane & 0.23 & 0.21 & 0.23 & 0.24 \\
Nonane & 0.24 & 0.22 & 0.24 & 0.26 \\
Decane & 0.26 & 0.23 & 0.26 & 0.28 \\
\hline
\end{tabular}

The probabilites of conformers of hexane with different numbers of gauche bonds are further broken down in Table 3.14. Here the probability of each of the significantly populated conformers is listed. The primary feature to note is that for a given number of gauche bonds, regardless of their placement, the probabilities of the conformers are very similar. This further shows that the solute-solvent interaction has little effect on the conformer probabilities. Finally, it should be noted that the approximate probability of a given conformer can be determined from Table 3.13 by taking the probability for conformers with the same number of gauche bonds and dividing by the number of conformers with that number of gauche bonds. Because of this and the large number of conformers sampled by the longer alkanes, detailed tables of conformer probabilites like Table 3.14 will not be presented for the longer alkanes. 
Table 3.13: Conformer probabilities from two-adjustable-parameter fits to experimental dipole couplings.

\begin{tabular}{|c|c|c|c|c|c|c|c|c|c|c|}
\hline & \multirow{3}{*}{$\begin{array}{c}\text { No. } \\
\text { Gauche } \\
\text { Bonds }\end{array}$} & \multirow{3}{*}{$\begin{array}{l}\text { No. } \\
\text { Confs }^{\dagger}\end{array}$} & \multicolumn{8}{|c|}{ Probabilities } \\
\hline & & & \multicolumn{2}{|c|}{ Model A } & \multicolumn{2}{|c|}{ Model B } & \multicolumn{2}{|c|}{ Model C } & \multicolumn{2}{|c|}{ Model D } \\
\hline & & & Model & Iso. & Model & Iso. & Model & Iso. & Model & Iso. \\
\hline Hexane & 0 & 1 & 0.27 & 0.29 & 0.28 & 0.27 & 0.27 & 0.26 & 0.20 & 0.19 \\
\hline 27 & 1 & 6 & 0.50 & 0.50 & 0.50 & 0.50 & 0.50 & 0.50 & 0.50 & 0.50 \\
\hline Confs. & 2 & 8 & 0.21 & 0.19 & 0.20 & 0.21 & 0.21 & 0.22 & 0.27 & 0.28 \\
\hline Total & 3 & 2 & 0.02 & 0.01 & 0.02 & 0.02 & 0.02 & 0.02 & 0.03 & 0.03 \\
\hline Heptane & 0 & 1 & 0.20 & 0.22 & 0.22 & 0.21 & 0.20 & 0.19 & 0.13 & 0.12 \\
\hline 81 & 1 & 8 & 0.45 & 0.46 & 0.46 & 0.46 & 0.45 & 0.45 & 0.42 & 0.41 \\
\hline Confs. & 2 & 18 & 0.29 & 0.27 & 0.27 & 0.28 & 0.29 & 0.30 & 0.35 & 0.37 \\
\hline \multirow[t]{2}{*}{ Total } & 3 & 12 & 0.05 & 0.05 & 0.05 & 0.05 & 0.05 & 0.06 & 0.09 & 0.10 \\
\hline & 4 & 2 & 0.00 & 0.00 & 0.00 & 0.00 & 0.00 & 0.00 & 0.01 & 0.01 \\
\hline Octane & 0 & 1 & 0.16 & 0.18 & 0.19 & 0.18 & 0.17 & 0.16 & 0.09 & 0.07 \\
\hline 243 & 1 & 10 & 0.40 & 0.41 & 0.42 & 0.42 & 0.41 & 0.41 & 0.33 & 0.32 \\
\hline Confs. & 2 & 32 & 0.33 & 0.31 & 0.30 & 0.31 & 0.31 & 0.32 & 0.39 & 0.40 \\
\hline \multirow[t]{3}{*}{ Total } & 3 & 38 & 0.10 & 0.09 & 0.08 & 0.08 & 0.09 & 0.10 & 0.16 & 0.18 \\
\hline & 4 & 16 & 0.01 & 0.01 & 0.01 & 0.01 & 0.01 & 0.01 & 0.03 & 0.03 \\
\hline & 5 & 2 & 0.00 & 0.00 & 0.00 & 0.00 & 0.00 & 0.00 & 0.00 & 0.00 \\
\hline Nonane & 0 & 1 & 0.13 & 0.14 & 0.16 & 0.15 & 0.14 & 0.13 & 0.05 & 0.04 \\
\hline 729 & 1 & 12 & 0.35 & 0.37 & 0.39 & 0.38 & 0.36 & 0.36 & 0.25 & 0.23 \\
\hline Confs. & 2 & 50 & 0.35 & 0.34 & 0.32 & 0.33 & 0.34 & 0.35 & 0.38 & 0.38 \\
\hline \multirow[t]{4}{*}{ Total } & 3 & 88 & 0.14 & 0.13 & 0.11 & 0.12 & 0.14 & 0.14 & 0.24 & 0.26 \\
\hline & 4 & 66 & 0.02 & 0.02 & 0.02 & 0.02 & 0.02 & 0.02 & 0.07 & 0.07 \\
\hline & 5 & 20 & 0.00 & 0.00 & 0.00 & 0.00 & 0.00 & 0.00 & 0.01 & 0.01 \\
\hline & 6 & 2 & 0.00 & 0.00 & 0.00 & 0.00 & 0.00 & 0.00 & 0.00 & 0.00 \\
\hline Decane & 0 & 1 & 0.12 & 0.13 & 0.16 & 0.15 & 0.13 & 0.12 & 0.04 & 0.03 \\
\hline 2187 & 1 & 14 & 0.33 & 0.35 & 0.37 & 0.37 & 0.34 & 0.34 & 0.21 & 0.18 \\
\hline Confs. & 2 & 72 & 0.34 & 0.34 & 0.32 & 0.32 & 0.33 & 0.34 & 0.35 & 0.35 \\
\hline \multirow[t]{5}{*}{ Total } & 3 & 170 & 0.17 & 0.15 & 0.13 & 0.13 & 0.15 & 0.16 & 0.28 & 0.29 \\
\hline & 4 & 192 & 0.04 & 0.03 & 0.02 & 0.03 & 0.03 & 0.04 & 0.10 & 0.12 \\
\hline & 5 & 102 & 0.00 & 0.00 & 0.00 & 0.00 & 0.00 & 0.00 & 0.02 & 0.02 \\
\hline & 6 & 24 & 0.00 & 0.00 & 0.00 & 0.00 & 0.00 & 0.00 & 0.00 & 0.00 \\
\hline & 7 & 2 & 0.00 & 0.00 & 0.00 & 0.00 & 0.00 & 0.00 & 0.00 & 0.00 \\
\hline
\end{tabular}

${ }^{\dagger}$ Excluding conformers with adjacent gauche ${ }^{+}$gauche $^{-}$bonds. 
Table 3.14: Conformer probabilities for hexane from two-adjustable-parameter fits to experimental dipole couplings.

\begin{tabular}{|c||c|c|c|c|c|c|c|c|}
\hline \multirow{2}{*}{\multicolumn{1}{c||}{$\begin{array}{c}\text { Conformer } \\
\text { (Multiplicity) }\end{array}$}} & \multicolumn{8}{|c|}{ Probabilities } \\
\cline { 2 - 9 } & \multicolumn{2}{|c|}{ Model A } & \multicolumn{2}{c|}{ Model B } & \multicolumn{2}{c|}{ Model C } & \multicolumn{2}{c|}{ Model D } \\
\hline \hline $\operatorname{ttt}(1)$ & 0.274 & 0.292 & 0.280 & 0.272 & 0.272 & 0.261 & 0.201 & 0.186 \\
$\operatorname{gtt}(4)$ & 0.084 & 0.084 & 0.084 & 0.084 & 0.083 & 0.084 & 0.085 & 0.085 \\
$\operatorname{tgt}(2)$ & 0.083 & 0.084 & 0.084 & 0.084 & 0.084 & 0.084 & 0.083 & 0.082 \\
$\operatorname{ggt}(4)$ & 0.026 & 0.024 & 0.025 & 0.026 & 0.026 & 0.027 & 0.033 & 0.035 \\
$\operatorname{gtg}(2)$ & 0.026 & 0.024 & 0.026 & 0.026 & 0.026 & 0.027 & 0.033 & 0.035 \\
$\operatorname{gtg}^{-}(2)$ & 0.026 & 0.024 & 0.025 & 0.026 & 0.027 & 0.027 & 0.033 & 0.034 \\
$\operatorname{ggg}(2)$ & 0.008 & 0.007 & 0.008 & 0.008 & 0.008 & 0.009 & 0.013 & 0.014 \\
\hline
\end{tabular}

\subsection{Results of Three-Adjustable-Parameter Mod-}

\section{eling}

The dipole couplings were also fit with Models A and D using three adjustable parameters. Two solute-solvent interaction parameters ( $k$ and $\zeta$ for Model $\mathrm{A}$, or $\tilde{w}_{0}$ and $\tilde{w}_{1}$ for Model D) and the effective trans-gauche energy difference, $E_{\mathrm{g}}^{\text {eff }}$, were used as adjustable parameters in the simplex minimization. Minimization with Model D reached a stable minimum, but no single minimum was found with Model A. There is a range of values for the adjustable parameters in Model A which result in fits of similar quality to the one shown in Table 3.15. This occurs because the two adjustable parameters, $k$ and $\zeta$, multiply the terms $c^{2}(\Omega)$ and $c(\Omega) z(\Omega)$, respectively, and $c(\Omega)$ and $z(\Omega)$ are not independent. Generally as $c(\Omega)$ decreases for a given molecule, $z(\Omega)$ increases, as shown in Figure 3.11. This interdependence yields a wide range of values for the adjustable parameters which provide fits of similar quality. Furthermore, Model A gives an unusually low value for $E_{\mathrm{g}}^{\mathrm{eff}}$. Model A is judged deficient, for while it fits the experimental couplings fairly well, the 
resulting adjustable parameters seem physically unreasonable.

Table 3.15: Results of three-adjustable-parameter fits to experimental dipole couplings.

\begin{tabular}{|r|r||r|r|r|r|r|}
\hline & & Hexane & Heptane & Octane & Nonane & Decane \\
\hline \hline \multirow{5}{*}{ Model A } & $\begin{array}{c}k \\
\left(10^{-3} \mathrm{Nm}^{-1}\right)\end{array}$ & -3.79 & -2.27 & -3.86 & -2.12 & -2.58 \\
\cline { 2 - 7 } & $\begin{array}{c}\zeta \\
\left(10^{-3} \mathrm{Nm}^{-1}\right)\end{array}$ & 74.76 & 56.86 & 78.94 & 55.42 & 66.54 \\
\cline { 2 - 7 } & $\begin{array}{c}E_{\mathrm{g}}^{\text {eff }} \\
\left(k J m^{-1}\right)\end{array}$ & 1.51 & 1.79 & 0.68 & 1.37 & 0.68 \\
\cline { 2 - 7 } & $\begin{array}{c}\text { RMS Dev. } \\
(\mathrm{Hz})\end{array}$ & 56 & 39 & 66 & 78 & 77 \\
\hline \multirow{5}{*}{ Model D } & $\begin{array}{c}\tilde{w}_{0} \\
\left(k J m o l^{-1}\right)\end{array}$ & 0.970 & 0.914 & 0.914 & 0.907 & 0.855 \\
\cline { 2 - 7 } & $\begin{array}{c}\tilde{w}_{1} \\
\left(k J m o l^{-1}\right)\end{array}$ & 0.598 & 0.538 & 0.550 & 0.494 & 0.500 \\
\cline { 2 - 7 } & $\begin{array}{c}E_{\mathbf{g}}^{\text {eff }} \\
\left(k J m o l^{-1}\right)\end{array}$ & 2.11 & 2.44 & 2.40 & 2.46 & 2.70 \\
\cline { 2 - 7 } & $\begin{array}{c}\text { RMS Dev. } \\
(\mathrm{Hz})\end{array}$ & 42 & 28 & 37 & 38 & 47 \\
\hline
\end{tabular}

Model $\mathrm{D}$, on the other hand, fits the dipole couplings better than Model A, as can be seen in Tables 3.15-3.20 and has physically reasonable values of the adjustable parameters that are not very different from those found in the two-parameter fit. $E_{\mathrm{g}}^{\text {eff }}$ stays almost the same as in the two-parameter fit, and when $\tilde{w}_{0}$ and $\tilde{w}_{1}$ are varied independently in the three-parameter fits, $\tilde{w}_{0}:_{3}$ a little higher and $\tilde{w}_{1}$ is a little lower than when they are varied together in the two-parameter fit. The quality of the fit using Model $D$ is closer to the experimental uncertainty of the dipole couplings of $\pm 25 \mathrm{~Hz}$. Better fits may be possible by extending the model and increasing the number of adjustable parameters as described in reference [1]. This will be investigated in Section 3.11 .

Order parameters for the alkanes can be calculated based on the best-fit values of 
Table 3.16: Dipole couplings for hexane from the three-adjustable-parameter fits to the experimental couplings.

\begin{tabular}{|c||r|r|r|}
\hline \multicolumn{1}{|c||}{} & \multicolumn{3}{c|}{ Dipole Couplings (Hz) } \\
\hline Protons & Experiment & Model A & Model D \\
\hline \hline $1-2$ & 1526 & 1484 & 1616 \\
$4-5$ & 3288 & 3386 & 3235 \\
$6-7$ & 3711 & 3604 & 3702 \\
$1-4$ & -310 & -262 & -361 \\
$1-6$ & -852 & -881 & -836 \\
$1-8$ & -484 & -511 & -540 \\
$1-10$ & -272 & -264 & -254 \\
$1-12$ & -167 & -160 & -158 \\
$4-8$ & -1323 & -1372 & -1323 \\
$4-9$ & -881 & -839 & -907 \\
$4-6$ & - & -77 & 131 \\
$4-7$ & - & 94 & 177 \\
$4-10$ & - & -751 & -720 \\
$4-11$ & - & -513 & -525 \\
$6-8$ & - & 17 & -151 \\
$6-9$ & - & 104 & -1 \\
\hline
\end{tabular}

the adjustable parameters and are listed in Table 3.21. The values for Model D with three adjustable parameters are slightly lower than with two adjustable parameters in Table 3.12, but do not differ dramatically. The conformer probabilities are listed in Table 3.22, and it can be seen that the values for the three-parameter fits using Model D differ only slightly from those found in the two-parameter fits in Table 3.13. For completeness, the probabilities of the different conformers of hexane for the three-parameter fits is presented in Table 3.23, though for Model D they are quite similar to the values found in the two-parameter fits. Thus Model D can be used with three adjustable parameters to better fit the experimental dipole couplings. Not only are the fits better than with any of the two-adjustable-parameter models, but the values of the adjustable parameters are well behaved in going from two to three adjustable parameters. All in all Model D appears to be a very good model for intermolecular interactions in liquid crystals and has adjustable parameters which 
Table 3.17: Dipole couplings for heptane from the three-adjustable-parameter fits to the experimental couplings.

\begin{tabular}{|c||r|r|r|}
\hline \multicolumn{1}{|c||}{} & \multicolumn{3}{c|}{ Dipole Couplings (Hz) } \\
\hline Protons & Experiment & Model A & Model D \\
\hline \hline $1-2$ & 1489 & 1399 & 1504 \\
$4-5$ & 3461 & 3412 & 3426 \\
$6-7$ & 3897 & 3905 & 3865 \\
$8-9$ & 4093 & 4135 & 4144 \\
$1-4$ & -226 & -215 & -289 \\
$1-6$ & -916 & -919 & -888 \\
$1-8$ & -520 & -544 & -541 \\
$1-10$ & -290 & -278 & -275 \\
$1-12$ & -192 & -189 & -182 \\
$1-14$ & -127 & -117 & -120 \\
$4-8$ & -1453 & -1489 & -1456 \\
$4-9$ & -924 & -903 & -923 \\
$6-10$ & -1575 & -1632 & -1582 \\
$6-11$ & -975 & -924 & -976 \\
$4-6$ & - & -76 & 25 \\
$4-7$ & - & 59 & 111 \\
$4-10$ & - & -755 & -772 \\
$4-11$ & - & -541 & -526 \\
$4-12$ & - & -304 & -294 \\
$4-13$ & - & -302 & -289 \\
$6-8$ & - & 65 & 17 \\
$6-9$ & - & 187 & 114 \\
\hline
\end{tabular}


Table 3.18: Dipole couplings for octane from the three-adjustable-parameter fits to the experimental couplings.

\begin{tabular}{|c||r|r|r|}
\hline \multicolumn{1}{|c||}{} & \multicolumn{3}{c|}{ Dipole Couplings (Hz) } \\
\hline Protons & Experiment & Model A & Model D \\
\hline \hline $1-2$ & 1637 & 1513 & 1683 \\
$4-5$ & 3539 & 3589 & 3458 \\
$6-7$ & 4119 & 4069 & 4101 \\
$8-9$ & 4319 & 4353 & 4384 \\
$1-4$ & -319 & -228 & -359 \\
$1-6$ & -949 & -969 & -912 \\
$1-8$ & -538 & -564 & -573 \\
$1-10$ & -296 & -301 & -289 \\
$1-12$ & -204 & -187 & -200 \\
$1-14$ & -129 & -127 & -138 \\
$1-16$ & -96 & -84 & -95 \\
$4-8$ & -1544 & -1539 & -1545 \\
$4-9$ & -976 & -941 & -993 \\
$6-10$ & -1710 & -1755 & -1720 \\
$6-11$ & -1088 & -913 & -1042 \\
$4-6$ & - & -147 & 149 \\
$4-7$ & - & 60 & 195 \\
$4-10$ & - & -848 & -843 \\
$4-11$ & - & -552 & -559 \\
$4-12$ & - & -330 & -333 \\
$4-13$ & - & -307 & -327 \\
$4-14$ & - & -218 & -205 \\
$4-15$ & - & -210 & -224 \\
$6-8$ & - & 73 & -106 \\
$6-9$ & - & 175 & 46 \\
$6-12$ & - & -698 & -880 \\
$6-13$ & - & -485 & -587 \\
$8-10$ & -155 & 204 \\
$8-11$ & & 186 & 236 \\
\hline
\end{tabular}


Table 3.19: Dipole couplings for nonane from the three-adjustable-parameter fits to the experimental couplings.

\begin{tabular}{|c|c|c|c|}
\hline & \multicolumn{3}{|c|}{ Dipole Couplings (Hz) } \\
\hline Protons & Experiment & Model A & Model D \\
\hline$\overline{1-2}$ & $\overline{1546}$ & 1392 & $\overline{1546}$ \\
\hline $4-5$ & 3570 & 3415 & 3490 \\
\hline $6-7$ & 4104 & 4067 & 4042 \\
\hline 8-9 & 4420 & 4465 & 4483 \\
\hline $10-11$ & 4420 & 4536 & 4494 \\
\hline $1-4$ & -265 & -218 & -300 \\
\hline $1-6$ & -958 & -929 & -906 \\
\hline $1-8$ & -539 & -559 & -558 \\
\hline $1-10$ & -300 & -290 & -289 \\
\hline $1-12$ & -206 & -198 & -198 \\
\hline $1-14$ & -138 & -130 & -140 \\
\hline $1-16$ & -93 & -93 & -101 \\
\hline $1-18$ & -73 & -65 & -72 \\
\hline $4-8$ & -1554 & -1570 & -1540 \\
\hline 4-9 & -977 & -961 & -957 \\
\hline $6-10$ & -1721 & -1835 & -1735 \\
\hline $6-11$ & -1028 & -967 & -1049 \\
\hline $8-12$ & -1779 & -1894 & -1776 \\
\hline $8-13$ & -1097 & -971 & -1049 \\
\hline 4-6 & - & -42 & 50 \\
\hline 4-7 & - & 105 & 130 \\
\hline $4-10$ & - & -793 & -826 \\
\hline $4-11$ & - & -554 & -550 \\
\hline $4-12$ & - & -339 & -331 \\
\hline $4-13$ & - & -325 & -323 \\
\hline $4-14$ & - & -217 & -212 \\
\hline $4-15$ & - & -214 & -230 \\
\hline $4-16$ & - & -141 & -150 \\
\hline $4-17$ & - & -140 & -149 \\
\hline $6-8$ & - & 56 & 9 \\
\hline $6-9$ & - & 204 & 118 \\
\hline $6-12$ & - & -816 & -901 \\
\hline $6-13$ & - & -505 & -574 \\
\hline $6-14$ & - & -343 & -354 \\
\hline $6-15$ & . & -323 & -346 \\
\hline $8-10$ & - & -39 & 62 \\
\hline $8-11$ & - & 188 & 151 \\
\hline
\end{tabular}

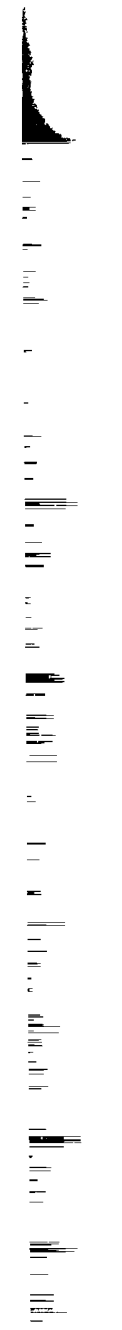


Table 3.20: Dipole couplings for decane from the three-adjustable-parameter fits to the experimental couplings.

\begin{tabular}{|c|c|c|c|}
\hline & \multicolumn{3}{|c|}{ Dipole Couplings (Hz) } \\
\hline Protons & Experiment & Model A & Model D \\
\hline$\overline{1-2}$ & 1669 & 1458 & 1705 \\
\hline 45 & 3660 & 3626 & 3542 \\
\hline $6-7$ & 4283 & 4298 & 4213 \\
\hline $8-9$ & 4577 & 4605 & 4617 \\
\hline $10-11$ & 4697 & 4708 & 4813 \\
\hline $1-4$ & -313 & -179 & -362 \\
\hline $1-6$ & -998 & -995 & -938 \\
\hline $1-8$ & -556 & -583 & -560 \\
\hline $1-10$ & -319 & -312 & -293 \\
\hline $1-12$ & -219 & -196 & -203 \\
\hline $1-14$ & -151 & -135 & -143 \\
\hline 1.16 & -127 & -90 & -105 \\
\hline $1-18$ & -82 & -67 & -78 \\
\hline $1-20$ & -57 & -48 & -58 \\
\hline $4-8$ & -1666 & -1658 & -1656 \\
\hline $4-9$ & -1020 & -1001 & -993 \\
\hline $6-10$ & -1863 & -1946 & -1884 \\
\hline $6-11$ & -1062 & -991 & -1070 \\
\hline $8-12$ & -1970 & -2026 & -1958 \\
\hline $8-13$ & -1140 & -954 & -1121 \\
\hline $4-6$ & - & -158 & 157 \\
\hline $4-7$ & - & 26 & 203 \\
\hline $4-10$ & - & -879 & -845 \\
\hline $4-11$ & - & -573 & -536 \\
\hline 4-12 & - & -356 & -351 \\
\hline 4-13 & - & -329 & -340 \\
\hline $4-14$ & - & -234 & -223 \\
\hline $4-15$ & - & -223 & -236 \\
\hline $4-16$ & - & -142 & -158 \\
\hline $4-17$ & - & -138 & -157 \\
\hline 4.18 & - & -101 & -112 \\
\hline 4-19 & - & -100 & -112 \\
\hline $6-8$ & - & 129 & -79 \\
\hline $6-9$ & - & 198 & 75 \\
\hline $6-12$ & - & -736 & -915 \\
\hline $6-13$ & - & -486 & -586 \\
\hline $6-14$ & - & -374 & -372 \\
\hline $6-15$ & - & -338 & -360 \\
\hline $6-16$ & - & -206 & -232 \\
\hline $6-17$ & - & -193 & $-2,45$ \\
\hline $8-10$ & - & -141 & 218 \\
\hline $8-11$ & - & 200 & 255 \\
\hline $8-14$ & - & -927 & -959 \\
\hline $8-15$ & - & -519 & -580 \\
\hline
\end{tabular}


have physically reasonable values and are well-behaved enough to extrapolate from these results to molecules of larger size.

Table 3.21: Order parameters from three-adjustable-parameter fits to experimental dipole couplings.

\begin{tabular}{|l||r|r|}
\hline \multicolumn{1}{|c||}{} & \multicolumn{2}{c|}{$S_{z z}$} \\
\cline { 2 - 3 } & Model A & Model D \\
\hline \hline Hexane & 0.18 & 0.18 \\
Heptane & 0.20 & 0.20 \\
Octane & 0.21 & 0.23 \\
Nonane & 0.23 & 0.24 \\
Decane & 0.24 & 0.26 \\
\hline
\end{tabular}

\subsection{Comparison of $n$-hexane in Liquid Crystals with $\Delta \chi>0$ and $\Delta \chi<0$}

As was mentioned in Chapter 2, nematic liquid crystals can have positive or negative diamagnetic anisotropy $(\Delta \chi)$. The two-proton filtered COSY experiments described in the previous sections used the liquid crystal EK11650, which has a positive diamagnetic anisotropy. It would be interesting to perform similar experiments in a liquid crystal with $\Delta \chi<0$. Such an experiment has been done with n-hexane dissolved in the liquid crystal Merck ZLI1167, a nematic liquid crystal composed of propyl, pentyl, and heptyl bicyclohexylcarbonitriles which has a negative diamagnetic anisotropy [50]. ZLI1167 has phase transitions from crystal to smectic $\mathrm{A}$ at $25^{\circ} \mathrm{C}$, from smectic $\mathrm{A}$ to nematic at $32^{\circ} \mathrm{C}$, and from nematic to isotropic liquid at $83^{\circ} \mathrm{C}$. $80 \%$ randomly deuterated $n$-hexane was dissolved in ZLI1167 to bring its concentration to $20 \mathrm{~mol} \%$. A two-proton filtered COSY experiment was 
Table 3.22: Conformer probabilities from three-adjustable-parameter fits to experimental dipole couplings.

\begin{tabular}{|c|c|c|c|c|c|c|}
\hline & \multirow{3}{*}{$\begin{array}{c}\text { No. } \\
\text { Gauche } \\
\text { Bonds }\end{array}$} & \multirow{3}{*}{$\begin{array}{c}\text { No. } \\
\text { Confs }^{\dagger}\end{array}$} & \multicolumn{4}{|c|}{ Probabilities } \\
\hline & & & \multicolumn{2}{|c|}{ Model A } & \multicolumn{2}{|c|}{ Model D } \\
\hline & & & Model & Iso. & Model & Iso. \\
\hline Hexane & $\overline{0}$ & 1 & 0.35 & 0.14 & 0.20 & 0.18 \\
\hline 27 & 1 & 6 & 0.47 & 0.46 & 0.50 & 0.50 \\
\hline Confs. & 2 & 8 & 0.17 & 0.35 & 0.27 & 0.28 \\
\hline Total & 3 & 2 & 0.01 & 0.05 & 0.03 & 0.03 \\
\hline Heptane & 0 & 1 & 0.23 & 0.09 & 0.14 & 0.13 \\
\hline 81 & 1 & 8 & 0.45 & 0.37 & 0.43 & 0.42 \\
\hline Confs. & 2 & 18 & 0.27 & 0.40 & 0.34 & 0.35 \\
\hline \multirow[t]{2}{*}{ Total } & 3 & 12 & 0.05 & 0.13 & 0.08 & 0.09 \\
\hline & 4 & 2 & 0.00 & 0.01 & 0.00 & 0.01 \\
\hline Octane & 0 & 1 & 0.23 & 0.02 & 0.09 & 0.08 \\
\hline 243 & 1 & 10 & 0.37 & 0.16 & 0.34 & 0.33 \\
\hline Confs. & 2 & 32 & 0.29 & 0.38 & 0.38 & 0.40 \\
\hline \multirow[t]{3}{*}{ Total } & 3 & 38 & 0.10 & 0.33 & 0.16 & 0.17 \\
\hline & 4 & 16 & 0.02 & 0.10 & 0.02 & 0.03 \\
\hline & 5 & 2 & 0.00 & 0.01 & 0.00 & 0.00 \\
\hline Nonane & 0 & 1 & 0.14 & 0.02 & 0.06 & 0.05 \\
\hline 729 & 1 & 12 & 0.34 & 0.14 & 0.26 & 0.25 \\
\hline Confs. & 2 & 50 & 0.32 & 0.34 & 0.38 & 0.39 \\
\hline \multirow[t]{4}{*}{ Total } & 3 & 88 & 0.16 & 0.33 & 0.23 & 0.24 \\
\hline & 4 & 66 & 0.03 & 0.14 & 0.06 & 0.07 \\
\hline & 5 & 20 & 0.00 & 0.02 & 0.01 & 0.01 \\
\hline & 6 & 2 & 0.00 & 0.00 & 0.00 & 0.00 \\
\hline Decane & 0 & 1 & 0.16 & 0.00 & 0.05 & 0.04 \\
\hline 2187 & 1 & 14 & 0.29 & 0.05 & 0.22 & 0.20 \\
\hline Confs. & 2 & 72 & 0.30 & 0.20 & 0.36 & 0.36 \\
\hline \multirow[t]{5}{*}{ Total } & 3 & 170 & 0.17 & 0.34 & 0.26 & 0.28 \\
\hline & 4 & 192 & 0.06 & 0.28 & 0.09 & 0.10 \\
\hline & 5 & 102 & 0.01 & 0.10 & 0.02 & 0.02 \\
\hline & 6 & 24 & 0.00 & 0.02 & 0.00 & 0.00 \\
\hline & 7 & 2 & 0.00 & 0.00 & 0.00 & 0.00 \\
\hline
\end{tabular}

$\dagger$ Excluding conformers with adjacent gauche ${ }^{+}$gauche $^{-}$bonds. 
Table 3.23: Conformer probabilities for hexane from three-adjustable-parameter fits to experimental dipole couplings.

\begin{tabular}{|c||c|c|c|c|}
\hline \multicolumn{1}{|c||}{\multirow{2}{*}{$\begin{array}{c}\text { Conformer } \\
\text { (Multiplicity) }\end{array}$}} & \multicolumn{4}{c|}{ Probabilities } \\
\cline { 2 - 5 } & \multicolumn{2}{|c|}{ Model A } & \multicolumn{2}{c|}{ Model D } \\
\hline \hline $\operatorname{ttt}(1)$ & 0.352 & 0.138 & 0.197 & 0.182 \\
$\operatorname{gtt}(4)$ & 0.069 & 0.077 & 0.084 & 0.085 \\
$\operatorname{tgt}(2)$ & 0.096 & 0.077 & 0.083 & 0.082 \\
$\operatorname{ggt}(4)$ & 0.020 & 0.013 & 0.034 & 0.036 \\
$\operatorname{gtg}(2)$ & 0.017 & 0.043 & 0.034 & 0.036 \\
$\operatorname{gtg}^{-}(2)$ & 0.025 & 0.043 & 0.034 & 0.035 \\
$\operatorname{ggg}(2)$ & 0.007 & 0.024 & 0.014 & 0.015 \\
\hline
\end{tabular}

done at $302 \mathrm{~K}$ and the dipole couplings between pairs of protons on $n$-hexane were determined. While the concentration and the reduced temperature were not the same as the previous experiments in EK11650, it should be possible to qualitatively compare the results for liquid crystal with $\Delta \chi>0$ and $\Delta \chi<0$. A comparison of the dipole couplings between pairs of protons on $n$-hexane dissolved in EK11650 and ZLI1167 is given in Table 3.24. The hexane/EK11650 results are from the experiments and modeling discussed in the previous section.

Some notable features of the ZLI1167 system are that the liquid crystal molecules tend to align with their long molecular axes perpendicular to the applied magnetic field. They have no macroscopic preferred direction in the plane perpendicular to the applied magnetic field, but tend to align parallel to neighboring molecules on a microscopic length scale. The main difference, though, is that measured dipole couplings in a liquid crystal with $\Delta \chi<0$ are scaled by a factor of $-\frac{1}{2}$ from those in a liquid crystal with $\Delta \chi>0$. It can be seen from Table 3.24 that this is approximately true, but since the liquid crystals and experimental conditions are different, the difference is not exactly a factor of $-\frac{1}{2}$. 
Table 3.24: Experimental and calculated dipole couplings for Hexane in the liquid crystals EK11650 and ZLI1167.

\begin{tabular}{|c||r|r|r|r|}
\hline \multicolumn{1}{|c||}{} & \multicolumn{4}{c|}{$\mathrm{D}(\mathrm{Hz})^{\ddagger}$} \\
\hline \multirow{3}{*}{ Protons $^{\dagger}$} & EK11650 & \multicolumn{2}{|c|}{ ZLI1167 } \\
\cline { 2 - 5 } & Experiment & Model D & Experiment & Model D \\
\hline \hline $1-2$ & 1526 & 1616 & -1119 & -1153 \\
$4-5$ & 3288 & 3235 & -2300 & -2273 \\
$6-7$ & 3711 & 3702 & -2611 & -2612 \\
$1-4$ & -310 & -361 & 221 & 246 \\
$1-6$ & -852 & -836 & 637 & 624 \\
$1-8$ & -484 & -540 & 353 & 384 \\
$1-10$ & -272 & -254 & 193 & 184 \\
$1-12$ & -167 & -158 & 120 & 116 \\
$4-8$ & -1323 & -1323 & 1013 & 1018 \\
$4-9$ & -881 & -907 & 652 & 663 \\
$4-6$ & - & 131 & - & -118 \\
$4-7$ & - & 177 & - & -138 \\
$4-10$ & - & -720 & - & 514 \\
$4-11$ & - & -525 & - & 367 \\
$6-8$ & - & -151 & - & 68 \\
$6-9$ & - & -1 & - & -27 \\
\hline
\end{tabular}

${ }^{\dagger}$ Labeling of protons is given in Figure 3.6

$\ddagger$ Experimental Error $\pm 25 \mathrm{~Hz}$ 
Model $\mathrm{D}$ with three adjustable parameters was judged to be best of the models tested in describing the alkane-liquid crystal interaction and was used to calculate dipole couplings for hexane in these two liquid crystals. Table 3.25 shows the results. The model did a very good job of fitting the couplings for hexane in ZLI1167, with the RMS deviation less than the experimental error of $\pm 25 \mathrm{~Hz}$, but it was less effective in modeling hexane in EK11650. The values of $E_{\mathbf{g}}^{\text {eff }}$ are very similar for the two liquid crystals, but the values of $\tilde{w}_{0}$ and $\tilde{w}_{1}$ are very different. The explanation for this can be seen in Table 3.26 where the order parameters for $n$-hexane in the two liquid crystals are given. Hexane is ordered to a much greater extent in the liquid crystal ZLI1167, and this may account for the difference in the values of $\tilde{w}_{0}$ and $\tilde{w}_{1}$ for the two systems. The similarity of the values of $E_{\mathrm{g}}^{\text {eff }}$ for hexane in the two liquid crystals shows that Model D does a good job of modeling the anisotropic soluteliquid crystal interaction, since the value of $E_{\mathrm{g}}^{\text {eff }}$ does not depend on the degree of ordering; the effects of the liquid crystal ordering are entirely accounted for by the anisotropic solute-liquid crystal interaction. The conformer probabilities are given in Table 3.27. It can be seen from this table that the effect of the liquid crystal solvent ZLI1167 is to make the elongated conformers (ttt, for example) noticibly more probable than for isotropic liquid hexane and more probable than in the less ordered liquid crystal EK11650.

In summary, the liquid crystals EK11650 and ZLI1167 with opposite signs of diamagnetic anisotropies appear to have ordering mechanisms which are well-described by Model $\mathrm{D}$. Hexane dissolved in the two liquid crystals seems to experience similar isotropic "solvent pressures", as reflected in the values of $E_{\mathrm{g}}^{\text {eff }}$, but the greater ordering effect of ZLI1167 makes elongated conformers like ttt slightly more probable 
than in EK11650 and more probable than in an isotropic liquid.

Table 3.25: Results of three-adjustable-parameter fits to experimental dipole couplings of hexane in EK11650 and hexane in ZLI1167.

\begin{tabular}{|c|r||r|r|}
\hline & & EK11650 & ZL[1167 \\
\hline \hline \multirow{5}{*}{ Model D } & $\begin{array}{c}\tilde{w}_{0} \\
\left(k J m l^{-1}\right)\end{array}$ & 0.970 & 1.168 \\
\cline { 2 - 4 } & $\begin{array}{c}\tilde{w}_{1} \\
\left(k J m o m^{-1}\right)\end{array}$ & 0.598 & 0.912 \\
\cline { 2 - 4 } & $\begin{array}{c}E_{\mathrm{g}}^{\text {eff }} \\
\left(k J m o l^{-1}\right)\end{array}$ & 2.11 & 2.22 \\
\cline { 2 - 4 } & $\begin{array}{c}\text { RMS Dev. } \\
(\mathrm{Hz})\end{array}$ & 42 & 20 \\
\hline
\end{tabular}

Table 3.26: Order parameters for hexane in EK11650 and ZLI167.

\begin{tabular}{|r||r|r|}
\hline \multicolumn{1}{|c||}{} & \multicolumn{2}{c|}{$S_{z z}$} \\
\cline { 2 - 3 } & EK11650 & ZLI167 \\
\hline \hline Hexane & 0.18 & 0.26 \\
\hline
\end{tabular}

Table 3.27: Conformer probabilities for hexane in EK11650 and ZLI1167.

\begin{tabular}{|c||r|r|r|r|}
\hline \multicolumn{1}{|c||}{\multirow{2}{*}{$\begin{array}{c}\text { Conformer } \\
\text { (Multiplicity) }\end{array}$}} & \multicolumn{3}{c|}{ Probabilities } \\
\cline { 2 - 5 } & \multicolumn{2}{|c|}{ EK11650 } & \multicolumn{2}{c|}{ ZLI1167 } \\
\hline \hline $\operatorname{ttt}(1)$ & 0.197 & 0.182 & 0.228 & 0.199 \\
$\operatorname{gtt}(4)$ & 0.084 & 0.085 & 0.084 & 0.086 \\
$\operatorname{tgt}(2)$ & 0.083 & 0.082 & 0.084 & 0.083 \\
$\operatorname{ggt}(4)$ & 0.034 & 0.036 & 0.030 & 0.034 \\
$\operatorname{gtg}(2)$ & 0.034 & 0.036 & 0.030 & 0.033 \\
$\operatorname{gtg}^{-}(2)$ & 0.034 & 0.035 & 0.031 & 0.033 \\
$\operatorname{ggg}(2)$ & 0.014 & 0.015 & 0.011 & 0.013 \\
\hline
\end{tabular}




\subsection{The Complete Set of Dipole Couplings for}

\section{Hexane}

While it isn't possible to determine the complete set of couplings for the longer alkanes, it is possible to do this for $n$-hexane. The experiments on $n$-hexane in EK11650 from Section 3.3 and on $n$-hexane in ZLI1167 from Section 3.9 were analyzed further to determine all 16 possible dipole couplings between pairs of protons on $n$-hexane. The couplings are listed in Table 3.28 along with the couplings calculated from Model $\mathrm{D}$ with three adjustable parameters. The results of the modeling are listed in Tables 3.29, 3.30, and 3.31. The effect of the additional dipole couplings does not seem to be very dramatic. It appears that the subset of dipole couplings used in the previous sections for the alkanes $n$-hexane through $n$-decane describe the alkanes sufficiently well. The uncertainty in assignment and sign of the additional dipole couplings $[36,37,38]$ may also affect these results, however.

\subsection{Further Studies with the Chord Model}

The chord model as used in the previous sections only takes into account carboncarbon bond self-correlations and neighboring carbon-carbon bond correlations. 'The model, as originally proposed, takes into account correlations between all possible pairs of carbon-carbon bonds, so that the tensor in Equation 3.28 can be written [1] :

$$
u_{a b}=-\frac{2}{3} \sum_{j=0}^{N-1} \sum_{i=1}^{N} \tilde{w}_{j} \frac{1}{2}\left[3\left(\frac{s_{a}^{i} s_{b}^{i+j}+s_{a}^{i+j} s_{b}^{i}}{2}\right)-\delta_{a b} s^{i} \cdot \mathbf{s}^{i+j}\right]
$$


Table 3.28: All 16 Experimental and calculated dipole couplings for hexane in the liquid crystals EK11650 and ZLI1167.

\begin{tabular}{|c||r|r|r|r|}
\hline \multicolumn{1}{|c||}{} & \multicolumn{4}{c|}{ Dipole Couplings (Hz) $)^{\ddagger}$} \\
\hline \multirow{2}{*}{ Protons $^{\dagger}$} & \multicolumn{2}{|c|}{ EK11650 } & \multicolumn{2}{|c|}{ ZLI1167 } \\
\cline { 2 - 5 } & Experiment & Model D & Experiment & Model D \\
\hline \hline $1-2$ & 1526 & 1619 & -1119 & -1161 \\
$4-5$ & 3288 & 3229 & -2300 & -2275 \\
$6-7$ & 3711 & 3685 & -2611 & -2602 \\
$1-4$ & -310 & -369 & 221 & 256 \\
$1-6$ & -852 & -828 & 637 & 615 \\
$1-8$ & -484 & -522 & 353 & 370 \\
$1-10$ & -272 & -248 & 193 & 179 \\
$1-12$ & -167 & -154 & 120 & 113 \\
$4-8$ & -1323 & -1325 & 1013 & 1011 \\
$4-9$ & -881 & -888 & 652 & 644 \\
$4-6$ & 56 & 139 & -79 & -124 \\
$4-7$ & 148 & 181 & -129 & -141 \\
$4-10$ & -585 & -701 & 425 & 499 \\
$4-11$ & -504 & -502 & 362 & 348 \\
$6-8$ & -157 & -163 & 121 & 85 \\
$6-9$ & 33 & -6 & -39 & -17 \\
\hline
\end{tabular}

${ }^{\dagger}$ Labeling of protons is given in Figure 3.6

$\ddagger$ Experimental Error $\pm 25 \mathrm{~Hz}$

Table 3.29: Results of three-adjustable-parameter fits to all 16 experimental dipole couplings of hexane in EK11650 and hexane in ZLI1167.

\begin{tabular}{|c|c||r|r|}
\hline & & EK11650 & ZLI1167 \\
\hline \hline \multirow{5}{*}{ Model D } & $\begin{array}{c}\tilde{w}_{0} \\
\left(k J \mathrm{~mol}^{-1}\right)\end{array}$ & 0.960 & 1.185 \\
\cline { 2 - 4 } & $\begin{array}{c}\tilde{w}_{1} \\
\left(k J \mathrm{~mol}^{-1}\right)\end{array}$ & 0.568 & 0.843 \\
\cline { 2 - 4 } & $\begin{array}{c}E_{\mathrm{g}}^{\text {eff }} \\
\left(\mathrm{kJmol}^{-1}\right)\end{array}$ & 2.25 & 2.34 \\
\cline { 2 - 4 } & $\begin{array}{c}\text { RMS Dev. } \\
(\mathrm{Hz})\end{array}$ & 51 & 30 \\
\hline
\end{tabular}


Table 3.30: Order parameters for hexane in EK11650 and ZLI167 using all 16 dipole couplings.

\begin{tabular}{|r||r|r|}
\hline \multicolumn{1}{|c||}{} & \multicolumn{2}{c|}{$S_{z z}$} \\
\cline { 2 - 3 } & EK11650 & ZLI1167 \\
\hline \hline Hexane & 0.18 & 0.26 \\
\hline
\end{tabular}

Table 3.31: Conformer probabilities for hexane in EK11650 and ZLI1167 using all 16 dipole couplings.

\begin{tabular}{|c||r|r|r|r|}
\hline \multicolumn{1}{|c||}{\multirow{2}{*}{$\begin{array}{c}\text { Conformer } \\
\text { (Multiplicity) }\end{array}$}} & \multicolumn{3}{c|}{ Probabilities } \\
\cline { 2 - 5 } & \multicolumn{2}{c|}{ EK11650 } & \multicolumn{2}{c|}{ ZLI1167 } \\
\hline \hline $\operatorname{ttt}(1)$ & 0.207 & 0.193 & 0.238 & 0.209 \\
$\operatorname{gtt}(4)$ & 0.085 & 0.085 & 0.084 & 0.086 \\
$\operatorname{tgt}(2)$ & 0.084 & 0.083 & 0.084 & 0.083 \\
$\operatorname{ggt}(4)$ & 0.033 & 0.034 & 0.029 & 0.032 \\
$\operatorname{gtg}(2)$ & 0.033 & 0.034 & 0.029 & 0.032 \\
$\operatorname{gtg}^{-}(2)$ & 0.033 & 0.033 & 0.030 & 0.031 \\
$\left.\operatorname{ggg}^{-12}\right)$ & 0.013 & 0.013 & 0.010 & 0.012 \\
\hline
\end{tabular}

where the symbols have the same meaning as in Equation 3.28. It was found in previous studies $[1,35,51]$ that the terms in Equation 3.30 with $j>1$ didn't contribute significantly to the ordering mechanism, and these terms were dropped. To check the validity of this assumption, the term with $j=2$ was also kept, and modeling of the series of alkanes in EK11650 and hexane in ZLI1167 was done with four adjustable parameters. The results are given in Table 3.32 and the calculated couplings are given in Tables 3.33-3.37 for this model.

It appears that the extra adjustable parameter improves the fits noticably, bringing them close to the level of experimental error. The value of $E_{\mathrm{g}}^{\text {eff }}$ stays nearly the same as in the two- and three-adjustable-parameter fits, and the order parameters (Table 3.38) and conformational probabilities (Tables 3.39 and 3.40) also are near the values found for the two- and three-adjustable-parameter fits. 
Table 3.32: Results of four-adjustable-parameter fits to experimental dipole couplings.

\begin{tabular}{|c||r|r|r|r|r|r|}
\hline & \multicolumn{2}{|c|}{ Hexane } & Heptane & Octane & Nonane & Decane \\
\cline { 2 - 7 } & EK11650 & ZLI1167 & & & & \\
\hline \hline $\begin{array}{c}\tilde{w}_{0} \\
\left(k J m o l^{-1}\right)\end{array}$ & 1.128 & 1.451 & 1.018 & 1.059 & 1.068 & 1.060 \\
\hline $\begin{array}{c}\tilde{w}_{1} \\
\left(k J m o l^{-1}\right)\end{array}$ & 0.673 & 1.031 & 0.586 & 0.630 & 0.579 & 0.610 \\
\hline $\begin{array}{c}\tilde{w}_{2} \\
\left(k J m o l^{-1}\right)\end{array}$ & -0.258 & -0.438 & -0.138 & -0.192 & -0.202 & -0.248 \\
\hline $\begin{array}{c}E_{\mathrm{g}}^{\text {eff }} \\
\left(k J m o l^{-1}\right)\end{array}$ & 1.93 & 1.94 & 2.28 & 2.20 & 2.26 & 2.42 \\
\hline $\begin{array}{c}\text { RMS Dev. } \\
(\mathrm{Hz})\end{array}$ & 39 & 15 & 24 & 28 & 23 & 28 \\
\hline
\end{tabular}

Table 3.33: Dipole couplings for hexane in the liquid crystals EK11650 and ZLI1167 from four-adjustable-parameter fits.

\begin{tabular}{|c||r|r|r|r|}
\hline \multicolumn{1}{|c||}{} & \multicolumn{4}{c|}{ Dipole Couplings (Hz) } \\
\hline \multirow{2}{*}{ Protons } & \multicolumn{2}{|c|}{ EK11650 } & \multicolumn{2}{c|}{ ZLI1167 } \\
\cline { 2 - 5 } & Experiment & Model D & Experiment & Model D \\
\hline \hline $1-2$ & 1526 & 1610 & -1119 & -1140 \\
$4-5$ & 3288 & 3266 & -2300 & -2298 \\
$6-7$ & 3711 & 3675 & -2611 & -2594 \\
$1-4$ & -310 & -356 & 221 & 239 \\
$1-6$ & -852 & -845 & 637 & 629 \\
$1-8$ & -484 & -530 & 353 & 377 \\
$1-10$ & -272 & -250 & 193 & 181 \\
$1-12$ & -167 & -151 & 120 & 113 \\
$4-8$ & -1323 & -1329 & 1013 & 1019 \\
$4-9$ & -881 & -917 & 652 & 669 \\
$4-6$ & - & 122 & - & -104 \\
$4-7$ & - & 170 & - & -129 \\
$4-10$ & - & -698 & - & 496 \\
$4-11$ & - & -517 & - & 362 \\
$6-8$ & - & -117 & - & 41 \\
$6-9$ & - & 16 & - & -41 \\
\hline
\end{tabular}


Table 3.34: Dipole couplings for heptane from the four-adjustable-parameter fits to the experimental couplings.

\begin{tabular}{|c||r|r|}
\hline \multicolumn{1}{|c||}{} & \multicolumn{2}{c|}{ Dipole Couplings (Hz) } \\
\hline Protons & Experiment & ModeI D \\
\hline \hline $1-2$ & 1489 & 1516 \\
$4-5$ & 3461 & 3457 \\
$6-7$ & 3897 & 3862 \\
$8-9$ & 4093 & 4110 \\
$1-4$ & -226 & -291 \\
$1-6$ & -916 & -897 \\
$1-8$ & -520 & -544 \\
$1-10$ & -290 & -275 \\
$1-12$ & -192 & -181 \\
$1-14$ & -127 & -120 \\
$4-8$ & -1453 & -1455 \\
$4-9$ & -924 & -938 \\
$6-10$ & -1575 & -1580 \\
$6-11$ & -975 & -980 \\
$4-6$ & - & 24 \\
$4-7$ & - & 110 \\
$4-10$ & - & -771 \\
$4-11$ & - & -529 \\
$4-12$ & - & -294 \\
$4-13$ & - & -290 \\
$6-8$ & - & 27 \\
$6-9$ & - & 118 \\
\hline
\end{tabular}


Table 3.35: Dipole couplings for octane from the four-adjustable-parameter fits to the experimental couplings.

\begin{tabular}{|c||r|r|}
\hline \multicolumn{1}{|c||}{} & \multicolumn{2}{c|}{ Dipole Couplings (Hz) } \\
\hline Protons & Experiment & Model D \\
\hline \hline $1-2$ & 1637 & 1699 \\
$4-5$ & 3539 & 3513 \\
$6-7$ & 4119 & 4097 \\
$8-9$ & 4319 & 4324 \\
$1-4$ & -319 & -358 \\
$1-6$ & -949 & -932 \\
$1-8$ & -538 & -580 \\
$1-10$ & -296 & -290 \\
$1-12$ & -204 & -199 \\
$1-14$ & -129 & -139 \\
$1-16$ & -96 & -96 \\
$4-8$ & -1544 & -1552 \\
$4-9$ & -976 & -1017 \\
$6-10$ & -1710 & -1715 \\
$6-11$ & -1088 & -1052 \\
$4-6$ & - & 141 \\
$4-7$ & - & 192 \\
$4-10$ & - & -842 \\
$4-11$ & - & -568 \\
$4-12$ & - & -334 \\
$4-13$ & - & -328 \\
$4-14$ & - & -201 \\
$4-15$ & - & -226 \\
$6-8$ & - & -79 \\
$6-9$ & - & 58 \\
$6-12$ & - & -884 \\
$6-13$ & - & -589 \\
$8-10$ & - & 210 \\
$8-11$ & & 238 \\
\hline
\end{tabular}


Table 3.36: Dipole couplings for nonane from the four-adjustable-parameter fits to the experimental couplings.

\begin{tabular}{|c|c|c|}
\hline & \multicolumn{2}{|c|}{ Dipole Couplings (Hz) } \\
\hline Protons & Experiment & Model D \\
\hline $1-2$ & $\overline{1546}$ & $\overline{1585}$ \\
\hline $4-5$ & 3570 & 3571 \\
\hline $6-7$ & 4104 & 4061 \\
\hline $8-9$ & 4420 & 4432 \\
\hline $10-11$ & 4420 & 4426 \\
\hline $1-4$ & -265 & -306 \\
\hline $1-6$ & -958 & -932 \\
\hline $1-8$ & -539 & -567 \\
\hline $1-10$ & -300 & -292 \\
\hline $1-12$ & -206 & -198 \\
\hline $1-14$ & -138 & -141 \\
\hline $1-16$ & -93 & -102 \\
\hline $1-18$ & -73 & -73 \\
\hline $4-8$ & -1554 & -1557 \\
\hline 4-9 & -977 & -988 \\
\hline $6-10$ & -1721 & -1739 \\
\hline $6-11$ & -1028 & -1063 \\
\hline $8-12$ & -1779 & -1769 \\
\hline $8-13$ & -1097 & -1062 \\
\hline 4-6 & - & 51 \\
\hline 4-7 & - & 133 \\
\hline $4-10$ & - & -828 \\
\hline $4-11$ & - & -559 \\
\hline $4-12$ & - & -333 \\
\hline $4-13$ & - & -325 \\
\hline $4-14$ & - & -208 \\
\hline $4-15$ & - & -232 \\
\hline $4-16$ & - & -150 \\
\hline $4-17$ & - & -150 \\
\hline $6-8$ & - & 29 \\
\hline $6-9$ & - & 127 \\
\hline $6-12$ & - & -904 \\
\hline $6-13$ & - & -579 \\
\hline $6-14$ & - & -354 \\
\hline $6-15$ & - & -346 \\
\hline $8-10$ & - & 79 \\
\hline $8-11$ & - & 159 \\
\hline
\end{tabular}


Table 2.37: Dipole couplings for decane from the four-adjustable-parameter fits to the experimental couplings.

\begin{tabular}{|c||r|r|}
\hline & \multicolumn{2}{|c|}{ Dipole Couplings (Hz) } \\
\hline Protons & Experiment & Model D \\
\hline \hline $1-2$ & 1669 & 1744 \\
$4-5$ & 3660 & 3645 \\
$6-7$ & 4283 & 4239 \\
$8-9$ & 4577 & 4561 \\
$10-11$ & 4697 & 4724 \\
$1-4$ & -313 & -364 \\
$1-6$ & -998 & -972 \\
$1-8$ & -556 & -575 \\
$1-10$ & -319 & -297 \\
$1-12$ & -219 & -205 \\
$1-14$ & -151 & -145 \\
$1-1$ & -127 & -107 \\
$1-18$ & -82 & -80 \\
$1-20$ & -57 & -59 \\
$4-8$ & -1666 & -1676 \\
$4-9$ & -1020 & -1035 \\
$6-10$ & -1863 & -1886 \\
$6-11$ & -1062 & -1093 \\
$8-12$ & -1970 & -1944 \\
$3-13$ & -1140 & -1141 \\
$4-6$ & - & 150 \\
$4-7$ & - & 202 \\
$4-10$ & - & -854 \\
$4-11$ & - & -555 \\
$4-12$ & - & -354 \\
$4-13$ & - & -344 \\
$4-14$ & - & -221 \\
$4-15$ & - & -240 \\
$4-16$ & - & -159 \\
$4-17$ & - & -159 \\
$4-18$ & - & -113 \\
$4-19$ & - & -114 \\
$6-8$ & - & -46 \\
$6-9$ & - & 90 \\
$6-12$ & - & -925 \\
$6-13$ & - & -597 \\
$6-14$ & - & -373 \\
$6-15$ & - & -362 \\
$6-16$ & - & -229 \\
$6-17$ & - & -249 \\
$8-10$ & - & 229 \\
$8-11$ & - & 259 \\
$8-14$ & - & -966 \\
$8-15$ & - & -595 \\
\hline & & \\
\hline
\end{tabular}


Table 3.38: Order parameters from Model D four-adjustable-parameter fits to experimental dipole couplings.

\begin{tabular}{|l||r|r|}
\hline \multicolumn{1}{|c||}{} & \multicolumn{2}{c|}{$S_{z z}$} \\
\cline { 2 - 3 } & EK11650 & ZLI1167 \\
\hline \hline Hexane & 0.18 & 0.26 \\
Heptane & 0.20 & - \\
Octane & 0.23 & - \\
Nonane & 0.24 & - \\
Decane & 0.26 & - \\
\hline
\end{tabular}

\subsection{Conclusions}

The work in this chapter has focussed on obtaining better experimental information about molecular conformation and modeling the conformation of flexible molecules in a constrained environment. Proton dipole couplings of flexible molecules dissolved in liquid crystals provide detailed information about the conformation and orientation of the flexible molecules not available by other experimental techniques. These experimental constraints give data with which to test models for intermolecular interactions in liquid crystals. In this chapter it was found that Model D proposed by Photinos, et al. [1] provides the best description of the anisotropic intermolecular interaction found in liquid crystals of the four mean-field models tested, in agreement with the results of Photinos, et. al. [51] for the proton dipole couplings of hexane. It also appears that the RIS model with an effective transgauche energy difference $\left(E_{\mathrm{g}}^{\text {eff }}\right)$ of $1.9-2.7 \mathrm{~kJ} \mathrm{~mol}^{-1}$ adequately describes the internal degrees of freedom and the isotropic "solvent pressure" on the alkane solutes. This range of values agrees well with other experimental measurements on alkanes in the liquid state [43]. Thus it can be concluded that alkyl chains in the $\mathrm{C}_{6}-\mathrm{C}_{10}$ range have conformational distributions which are not very different from those in the 
Table 3.39: Conformer probabilities from four-adjustable-parameter fits to experimental dipole couplings.

\begin{tabular}{|c|c|c|c|c|}
\hline & \multirow{3}{*}{$\begin{array}{c}\text { No. } \\
\text { Gauche } \\
\text { Bonds }\end{array}$} & \multirow{3}{*}{$\begin{array}{c}\text { No. } \\
\text { Confs }^{\dagger}\end{array}$} & \multirow{2}{*}{\multicolumn{2}{|c|}{$\frac{\text { Probabilities }}{\text { Model D }}$}} \\
\hline & & & & \\
\hline & & & Model & Iso. \\
\hline Hexane & 0 & 1 & 0.18 & 0.17 \\
\hline (EK11650) & 1 & 6 & 0.50 & 0.50 \\
\hline 27 Confs. & 2 & 8 & 0.29 & 0.30 \\
\hline Total & 3 & 2 & 0.03 & 0.03 \\
\hline Hexane & 0 & 1 & 0.21 & 0.18 \\
\hline (ZLI1167) & 1 & 6 & 0.50 & 0.50 \\
\hline 27 Confs. & 2 & 8 & 0.26 & 0.29 \\
\hline Total & 3 & 2 & 0.03 & 0.03 \\
\hline Heptane & 0 & 1 & 0.13 & 0.12 \\
\hline 81 & 1 & 8 & 0.42 & 0.41 \\
\hline Confs. & 2 & 18 & 0.35 & 0.37 \\
\hline Total & 3 & 12 & 0.09 & 0.09 \\
\hline & 4 & 2 & 0.01 & 0.01 \\
\hline Octane & 0 & 1 & 0.08 & 0.07 \\
\hline 243 & 1 & 10 & 0.32 & 0.31 \\
\hline Confs. & 2 & 32 & 0.39 & 0.40 \\
\hline Total & 3 & 38 & 0.18 & 0.19 \\
\hline & 4 & 16 & 0.03 & 0.03 \\
\hline & 5 & 2 & 0.00 & 0.00 \\
\hline Nonane & 0 & $\overline{1}$ & 0.05 & 0.04 \\
\hline 729 & 1 & 12 & 0.24 & 0.23 \\
\hline Confs. & 2 & 50 & 0.38 & 0.38 \\
\hline Total & 3 & 88 & 0.25 & 0.26 \\
\hline & 4 & 66 & 0.07 & 0.08 \\
\hline & 5 & 20 & 0.01 & 0.01 \\
\hline & 6 & 2 & 0.00 & 0.00 \\
\hline Decane & 0 & 1 & 0.04 & 0.03 \\
\hline 2187 & 1 & 14 & 0.20 & 0.18 \\
\hline Confs. & 2 & 72 & 0.35 & 0.35 \\
\hline Total & 3 & 170 & 0.28 & 0.30 \\
\hline & 4 & 192 & 0.11 & 0.12 \\
\hline & 5 & 102 & 0.02 & 0.02 \\
\hline & 6 & 24 & 0.00 & 0.00 \\
\hline & 7 & 2 & 0.00 & 0.00 \\
\hline
\end{tabular}

$\dagger$ Excluding conformers with adjacent gauche ${ }^{+}$gauche $^{-}$bonds. 
Table 3.40: Conformer probabilities for hexane in EK11650 and ZLI1167 from fouradjustable-parameter fits.

\begin{tabular}{|c||r|r|r|r|}
\hline \multicolumn{1}{|c||}{\multirow{2}{*}{$\begin{array}{c}\text { Conformer } \\
\text { (Multiplicity) }\end{array}$}} & \multicolumn{4}{c|}{ Probabilities } \\
\cline { 2 - 5 } & \multicolumn{2}{|c|}{ EK11650 } & \multicolumn{2}{c|}{ ZLI1167 } \\
\hline \hline $\operatorname{ttt}(1)$ & 0.183 & 0.168 & 0.208 & 0.175 \\
$\operatorname{gtt}(4)$ & 0.083 & 0.084 & 0.084 & 0.084 \\
$\operatorname{tgt}(2)$ & 0.082 & 0.081 & 0.082 & 0.081 \\
$\operatorname{ggt}(4)$ & 0.036 & 0.038 & 0.033 & 0.037 \\
$\operatorname{gtg}(2)$ & 0.036 & 0.038 & 0.033 & 0.037 \\
$\operatorname{gtg}(2)$ & 0.036 & 0.037 & 0.034 & 0.036 \\
$\operatorname{ggg}(2)$ & 0.016 & 0.017 & 0.014 & 0.016 \\
\hline
\end{tabular}

neat liquid, and that the liquid crystal solvent has only a slight effect on molecular conformation. 


\section{Chapter 4}

\section{NMR Studies of Molecular}

\section{Conformation and Orientation in}

\section{Graphite Intercalation}

\section{Compounds}

\subsection{Introduction}

Two Graphite Intercalation Compounds (GICs) composed of an alkali metal and tetrahydrofuran (THF) intercalated between the layers of highly-oriented pyrolytic graphite (HOPG) were studied by proton NMR to determine the conformation and orientation of the THF molecules in these compounds. These alkali/THF GICs are very different compounds than the alkane/liquid crystal mixtures studied in the previous chapter, but NMR studies of the compounds show that they have more in common than it first appears. Both compounds have conformationally and transla- 
tionally mobile species (alkanes or THF) which are in an anisotropic environment. Because of this, the NMR Hamiltonians are the same, and the same approach could be taken with the alkali/THF GICs as was taken with the alkanes in the previous chapter to determine the conformation and orientation of the THF. Random deuteration of the THF molecules and two-proton filtered NMR experiments should work well in this case for measuring dipole couplings between protons on a THF molecule. Unfortunately, this approach was not taken due to the unavailability of a sample with randomly deuterated THF. Instead, fully protonated THF was used, so that the NMR spectra are quite complex, resulting from eight-spin THF molecules. This makes it necessary to take a brute-force computational approach to simulation of the NMR spectra, since analytical calculation of the eigenstates and eigenvalues of such a spin system is prohibitively difficult. Before tackling the problem of interpreting the NMR spectra of THF in these GICs, a brief digression into some background about the compounds of interest is in order.

Graphite intercalation compounds (GICs) have been studied for many years $[52,53]$, both because of their possible applications (for example, as conductors) and as models for quasi-two-dimensional systems. Ternary GICs, reviewed recently by Solin and Zabel [54], are of special interest because they allow a wider variation of composition and properties than binary compounds.

Previous work on ternary alkali/THF GICs has revealed pronounced differences in their structure, depending on the type of intercalated alkali metal and on the amount of intercalated THF $[55,56]$. With the exception of compounds with $\operatorname{Li}[55$, 57 , the intercalants form monomolecular layers. In the case of the ternary $\mathrm{K}, \mathrm{Rb}$, or Cs/THF GICs, two forms of the GIC are possible - saturated and residual. The 
saturated form is a first-stage GIC (stage refers to the number of graphene layers between neighboring layers of intercalate) obtained by direct action of THF vapor on the second-stage binary GIC $\mathrm{MC}_{24}(\mathrm{M}=\mathrm{K}, \mathrm{Rb}, \mathrm{Cs})$. The residual compound is a first-stage GIC in the case of $\mathrm{K}$ or $\mathrm{Rb}$ and a second stage GIC for $\mathrm{Cs}$, and is formed by incomplete deintercalation of THF from the saturated one [58]. The intercalation process can be pictured by the pleated layer model of Daumas and Herold [59], shown schematically in Figure 4.1.

The differences in structure between ternary alkali/THF GICs containing different alkali metals is evident in their composition and in the spacing between the two nearest intercalated layers $\left(\mathrm{I}_{c}\right)$. The saturated form of the $\mathrm{K}$ or Rb/THF GIC has a $\mathrm{THF} /$ metal ratio of about 2.5 and an $\mathrm{I}_{c}$ of $8.9 \AA$, while the residual compound has a THF/metal ratio of about 1.6 and an $\mathrm{I}_{c}$ of $7.1 \AA[58,60]$. The saturated Cs/THF GIC is similar to the residual $\mathrm{K}$ or $\mathrm{Rb}$ compound and has a THF/Cs ratio of about 1.6 and an $I_{c}$ of $7.1 \AA$, and the residual compound is second stage with a THF/Cs ratio of about 0.9 and an $I_{c}$ value of $10.2 \AA[61,62]$.

The interlayer spacing for alkali/THF GICs is imposed by the size and orientation of the THF molecules, rather than by the size of the smaller alkali ions [58]. From the observed differences in composition and $\mathrm{I}_{c}$ between the $\mathrm{K}$ or $\mathrm{Rb} / \mathrm{THF}$ and Cs/THF saturated compounds, it was concluded that the mean plane of the THF molecule is approximately perpendicular to the graphene layers in the $\mathrm{K}$ and $\mathrm{Rb}$ compounds and parallel to the graphene layers in the Cs compound [62, 63, 64,65], as pictured in Figure 4.2 .

To better understand the differences between the $\mathrm{K}$ or $\mathrm{Rb} / \mathrm{THF}$ and $\mathrm{Cs} / \mathrm{THF}$ GICs, proton NMR studies have been conducted on saturated K/THF and satu- 


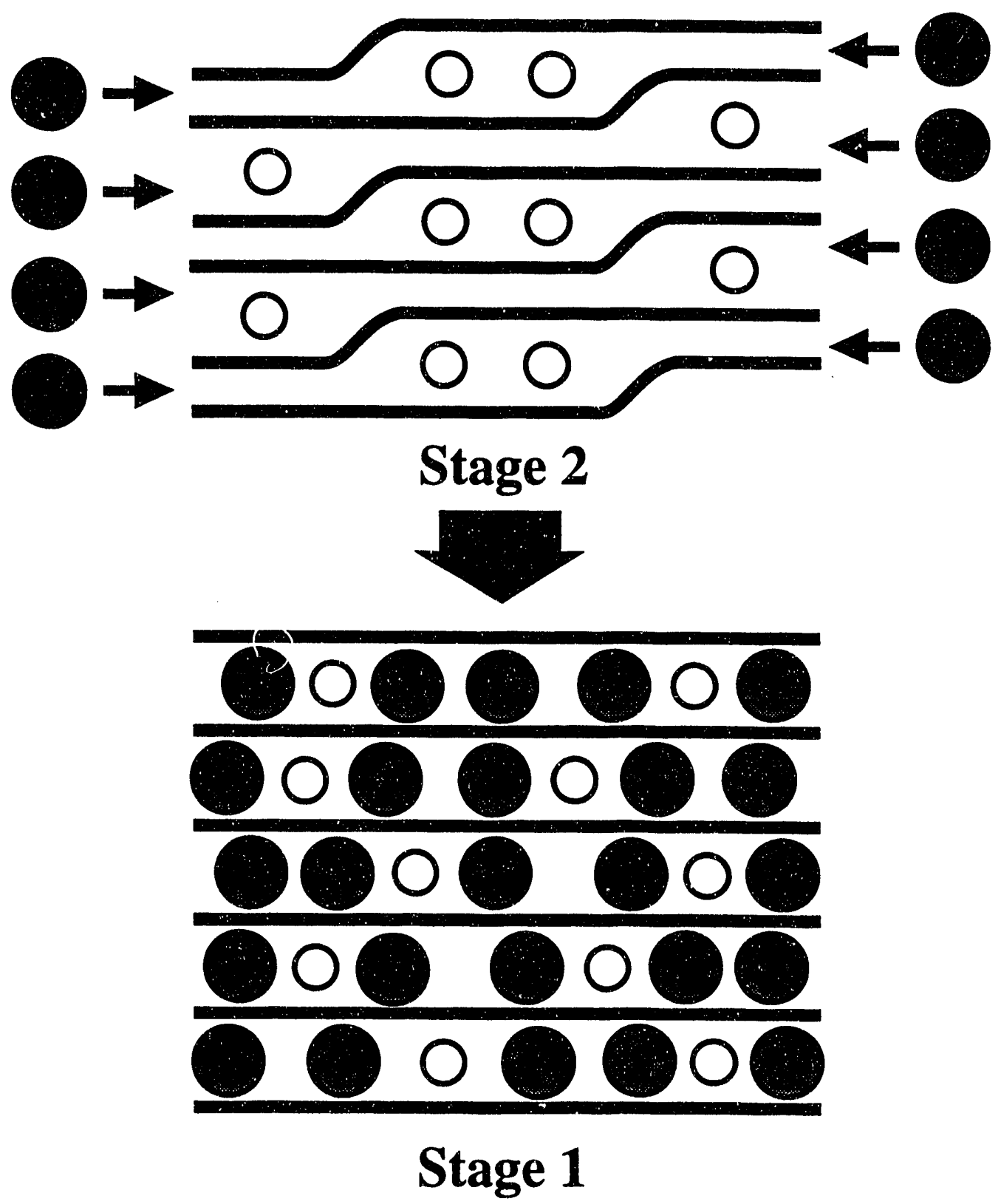

Figure 4.1: Pleated Layer Model for interclation of THF into the binary stage 2 alkali GIC to form the stage 1 ternary alkali/THF GIC. The open circles represent alkali metal ions and the filled circles represent THF molecules. 

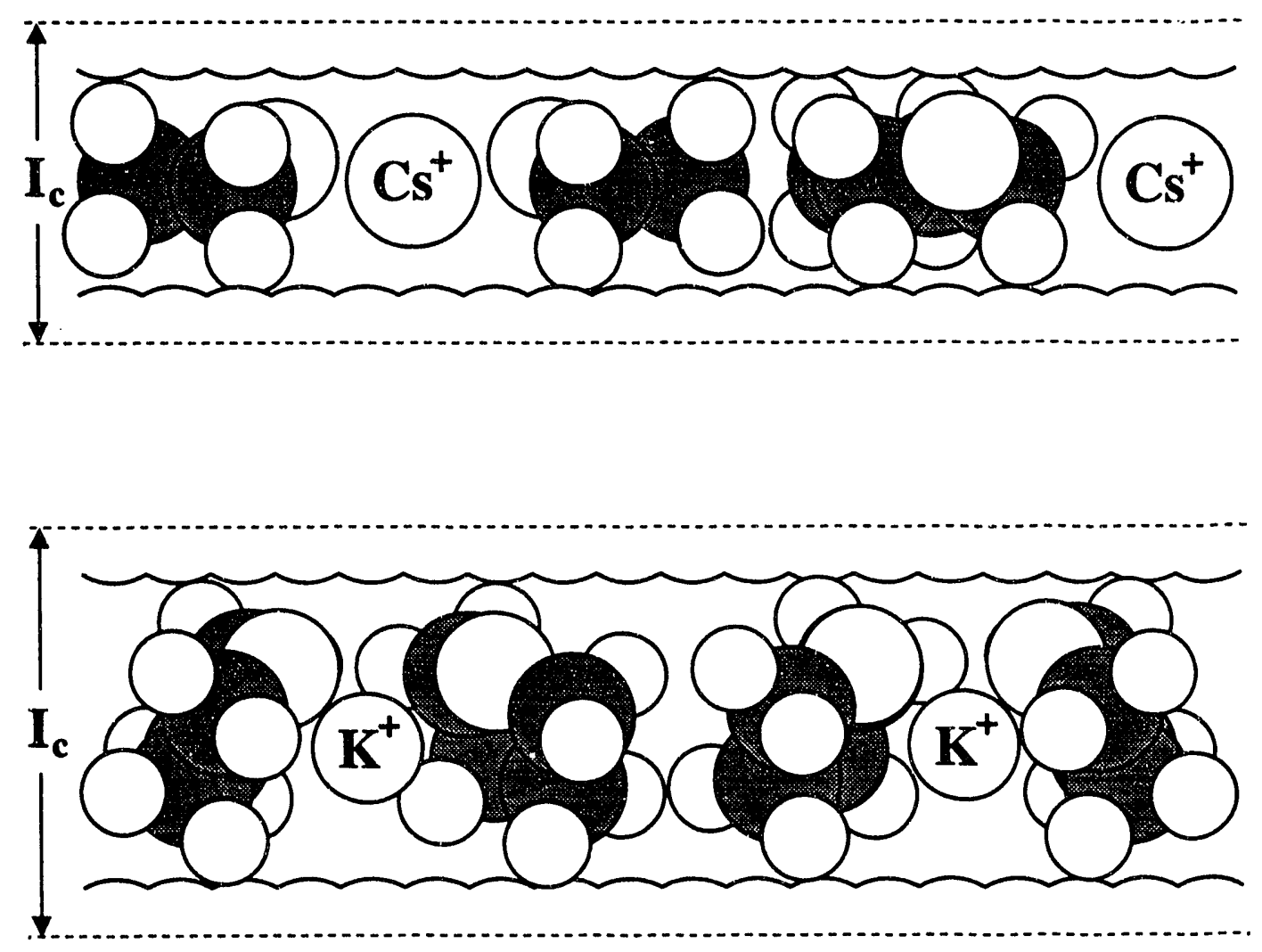

Figure 4.2: Schematic picture of the ternary graphite intercalation compounds $\mathrm{Cs}(\mathrm{THF})_{1.3} \mathrm{C}_{24}$ and $\mathrm{K}(\mathrm{THF})_{2.5} \mathrm{C}_{24}$. In $\mathrm{Cs}(\mathrm{THF})_{1.3} \mathrm{C}_{24}$ the THF molecules have their mean planes approximately parallel to the graphene layers, while in $\mathrm{K}(\mathrm{THF})_{2.5} \mathrm{C}_{24}$ they have their mean planes almost perpendicular to the graphene layers. 
rated Cs/THF GICs. Proton NMR spectroscopy is particularly informative here for two reasons. First, at room temperature, the observed lineshapes indicate that anisotropic molecular motions of the intercalated molecules effectively average out magnetic dipole-dipole couplings between spins on different molecules, leaving only intramolecular couplings. The dependence of these couplings on the orientation and length of proton-proton internuclear vectors provides information about molecular orientation, conformation, and mobility. Second, the layers of the graphite matrix impose a high degree of orientational order on the intercalated THF molecules. The situation is similar to that of molecules dissolved in, and partially oriented by, liquid crystals. But while NMR in liquid crystals has become a widely used method for structure determination, to date there have been only a few examples where dipolar spectra of molecules oriented in solids have resolved lines which permit detailed analysis (see for example, $[66,67]$ ). Thus NMR spectroscopy of intercalation compounds has been applied most often to powder samples $[68,69]$, which yield broad, unresolvable spectra. The almost perfectly parallel orientation of the graphene layers in the HOPG-GIC samples studied here, however, results in well-resolved NMR spectra.

Of special interest is the motion of the THF molecule itself. One possible mode of motion is rotation of the molecule around a fixed axis. In addition, as with other five-membered ring molecules, THF in the gas or liquid phase exhibits a large amplitude vibration known as pseudorotation $[70,71]$. One of the goals of this work is to determine whether or not such rotational and vibrational modes of THF are active in the intercalation compounds. 


\subsection{Experimental}

The preparation of ternary alkali/THF GICs has been described previously [64]. From measurement of THF consumption during intercalation, the THF/C ratio (referenced to $24 \mathrm{C}$ atoms) was determined to be 1.3 in the $\mathrm{Cs} / \mathrm{THF}$ sample, yielding a ternary sample of composition $\mathrm{Cs}_{x}(\mathrm{THF})_{1.3} \mathrm{C}_{24}$ with $x=1.0 \pm 0.1$. The amount of intercalated THF in this sample is less than that usually found in saturated Cs/THF GICs $[57,62,65]$, but the proton NMR spectrum of this sample is the same as that of a sample of saturated Cs/THF GIC with the higher THF/C $\mathrm{C}_{24}$ ratio of 1.6 [62]. The K/THF GIC has the approximate composition $\mathrm{K}_{x}(\mathrm{THF})_{2.5} \mathrm{C}_{24}$ with $x=1.0 \pm 0.1$.

NMR experiments on these two samples were performed on a Bruker CXP100 spectrometer operating at a proton frequercy of $100 \mathrm{MHz}$. The orientation of the sample inside the coil was adjusted by eye. The error in the angle $\Theta$ between the sample $c$ axis (perpendicular to the graphene layers) and the external magnetic field was estimated to be $\pm 2.5^{\circ}$ at $\Theta=0^{\circ}$ and $90^{\circ}$, and $\pm 10.0^{\circ}$ at intermediate angles.

The temperature dependence of the proton NMR spectra of the Cs/THF GIC is shown in Figure 4.3 for $\Theta=0^{\circ}$. At low temperature the spectrum is broad and featureless because the rate of motion of the THF molecules is greatly reduced. As the temperature increases, so does the mobility of the THF molecules, resulting in sharper spectral features due to averaging of intermolecular dipole couplings. The angular dependence of the proton spectra of both alkali/THF GICs at $293 \mathrm{~K}$ is shown in Figures 4.4 and 4.5. The overall width of the spectra shows the $3 \cos ^{2} \Theta$ - 1 dependence characteristic of dipolar spectra, but at angles between $0^{\circ}$ and $90^{\circ}$ individual lines in the spectra of the Cs/THF GIC appear to broaden more than 
those in the spectra of the K/THF GIC. This occurs because the graphene layers in the Cs/THF GIC exhibit a larger mosaic spread in orientation than those in the $\mathrm{K} / \mathrm{THF}$ compound. The orientational disorder of the graphene layers in the two samples is shown in the X-ray diffraction results in Figure 4.6.

\subsection{Simulation of the NMR Spectra}

Typically, all nuclear spins in a solid are dipole coupled to each other, resulting in a broad featureless spectrum like the low temperature spectrum of the Cs/THF GIC in Figure 4.3. At higher temperatures, however, the THF molecules are mobile; they rotate and probably undergo translational motion [62]. This rapid molecular motion results in a motionally-averaged Hamiltonian (Equation 1.48). The resolution of the room temperature spectra of the $\mathrm{Cs} / \mathrm{THF}$ and K/THF GICs in Figures 4.4 and 4.5 suggests that intermolecular dipole couplings are essentially averaged to zero and only the intramolecular couplings remain. Thus, to a good approximation, the room temperature spectra can be considered to result from isolated, eight-spin THF molecules, which can be simulated numerically. The spectrum of an eightspin system with $\mathrm{C}_{2 v}$ symmetry (possibly the symmetry of the time-averaged THF molecule) still has up to 2860 lines, leading to a crowded spectrum with extensive overlap of lines. Nevertheless, the spectra of THF in the highly oriented GICs studied here exhibit enough resolution and characteristic features to permit analysis.

To simulate the spectrum of THF, motional averaging must be carried out over the molecular motions of THF in the GIC. Possible motions include molecular rotatic sso and conformational changes or pseudorotation. THF was the first molecule for which spectroscopic evidence [72] was found for pseudorotation, a vibrational 

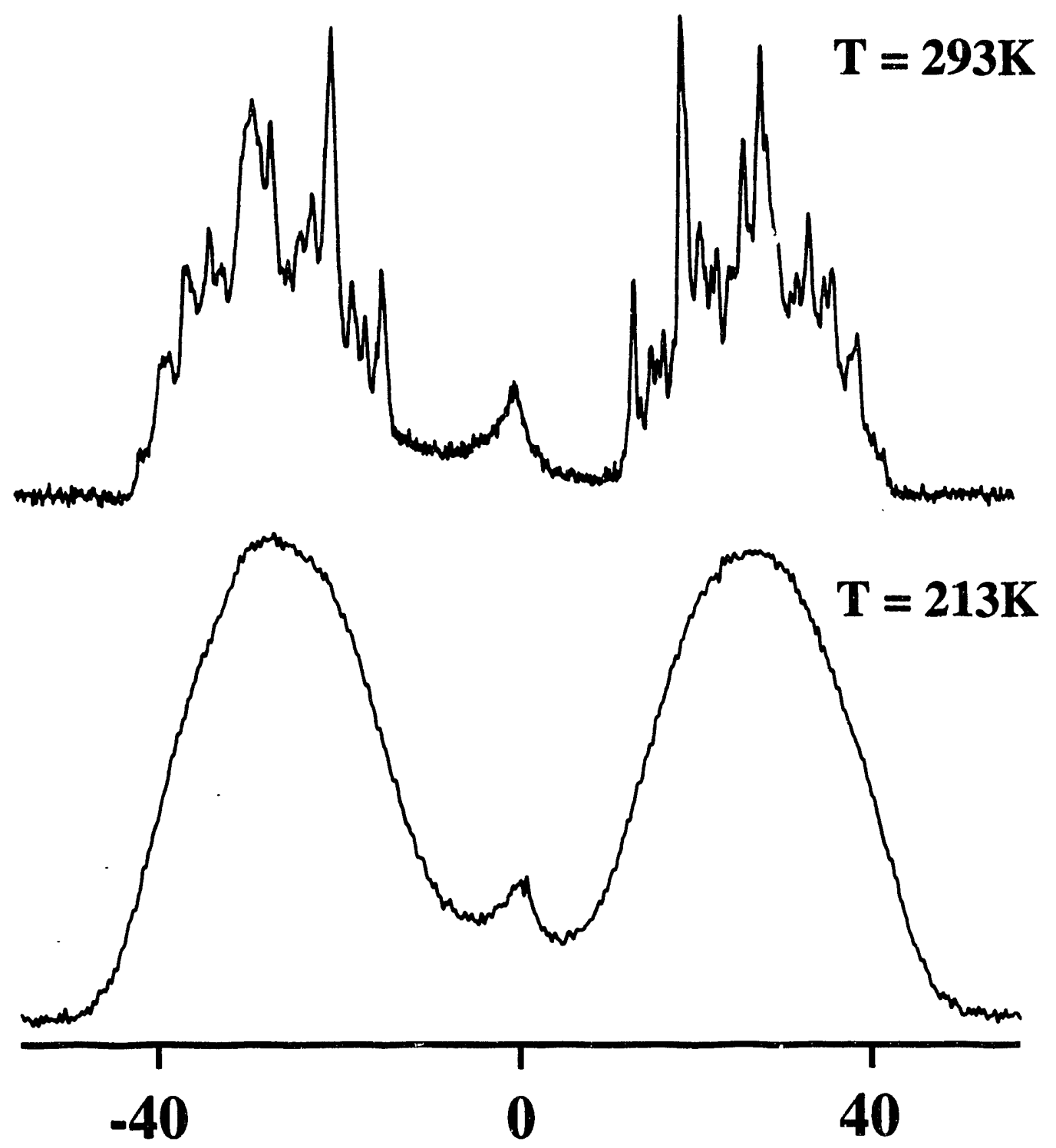

Frequency $(\mathbf{k H z})$

Figure 4.3: Experimental spectra of $\mathrm{Cs}(\mathrm{THF})_{1.3} \mathrm{C}_{24}$ with the graphene layers perpendicular to the external magnetic field. The narrower lines in the higher temperature spectrum result from an increase in the rates of motion of the THF molecules from $213 \mathrm{~K}$ to $293 \mathrm{~K}$. 


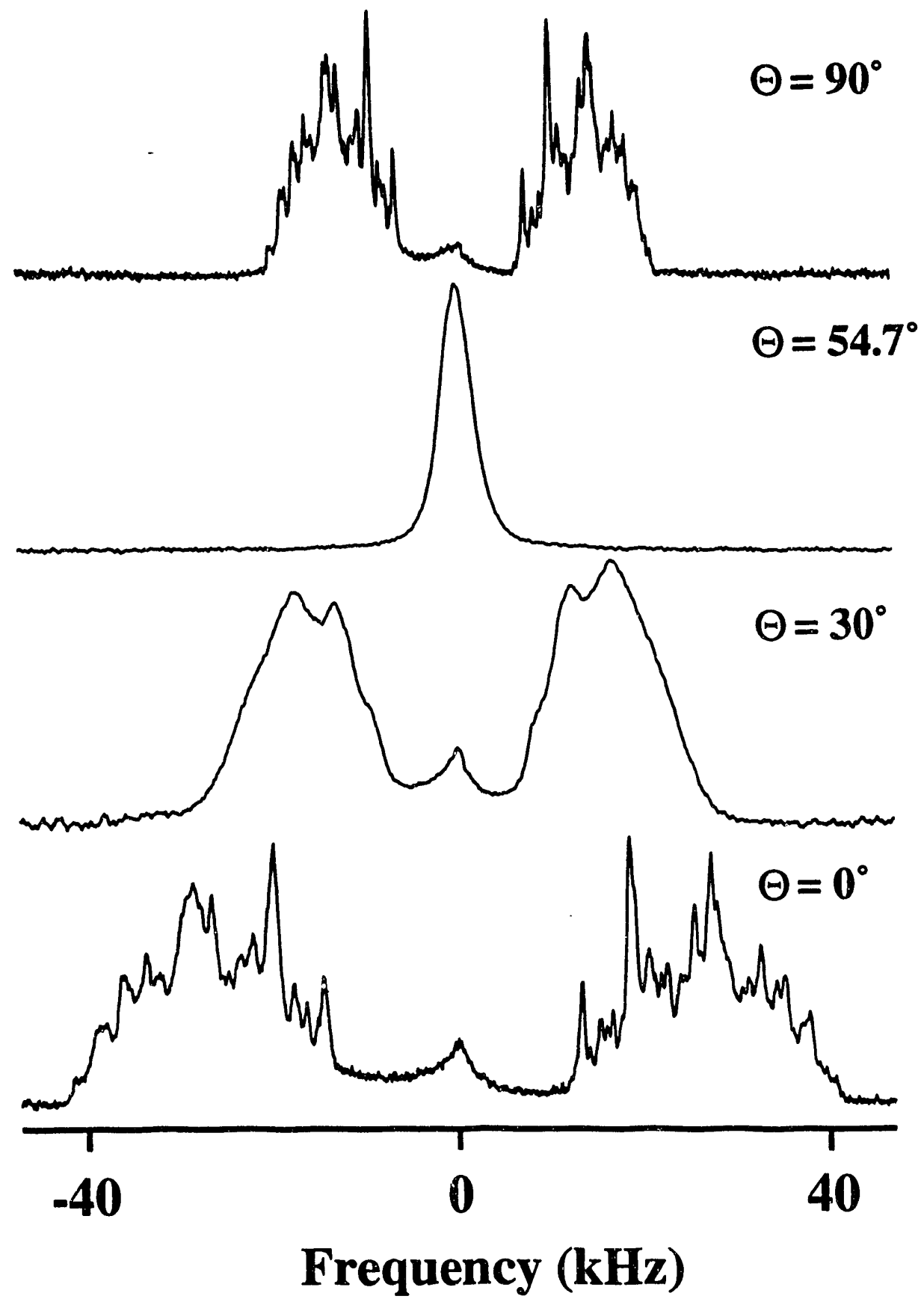

Figure 4.4: Experimental spectra of $\mathrm{Cs}(\mathrm{THF})_{1.3} \mathrm{C}_{24}$ for different angles $\theta$ between the normal to the graphene layers and the external magnetic field. The overall width of the features in the spectra scale as $3 \cos ^{2} \theta-1$ indicating rotation of THF about an axis perpendicular to the graphene layers. 


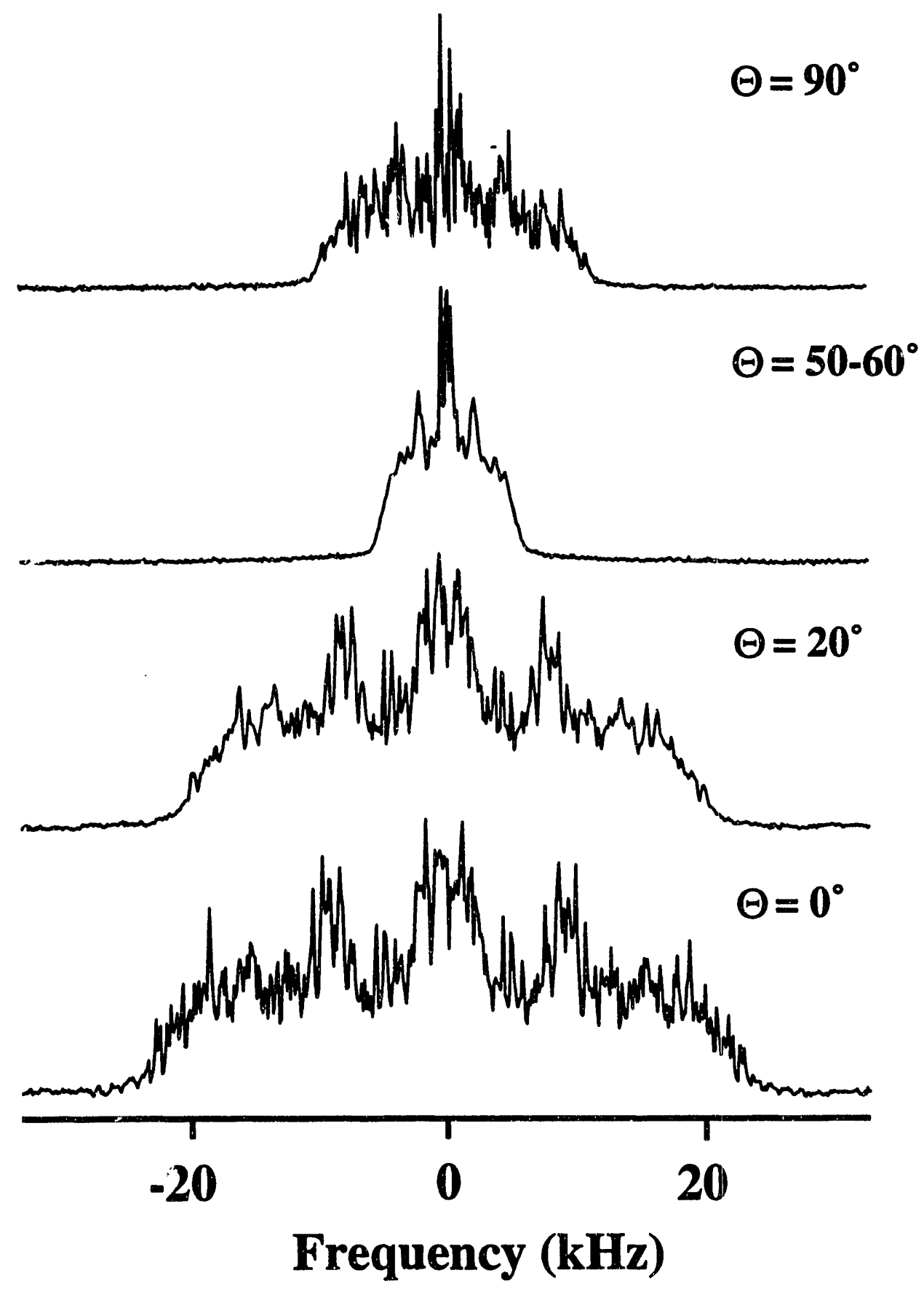

Figure 4.5: Experimental spectra of $\mathrm{K}(\mathrm{THF})_{2.5} \mathrm{C}_{24}$ for different angles $\theta$ between the normal to the graphene layers and the external magnetic field. The overall width of the features in the spectra scale as $3 \cos ^{2} \theta-1$ indicating rotation of $\mathrm{THF}$ about an axis perpendicular to the graphene layers. 


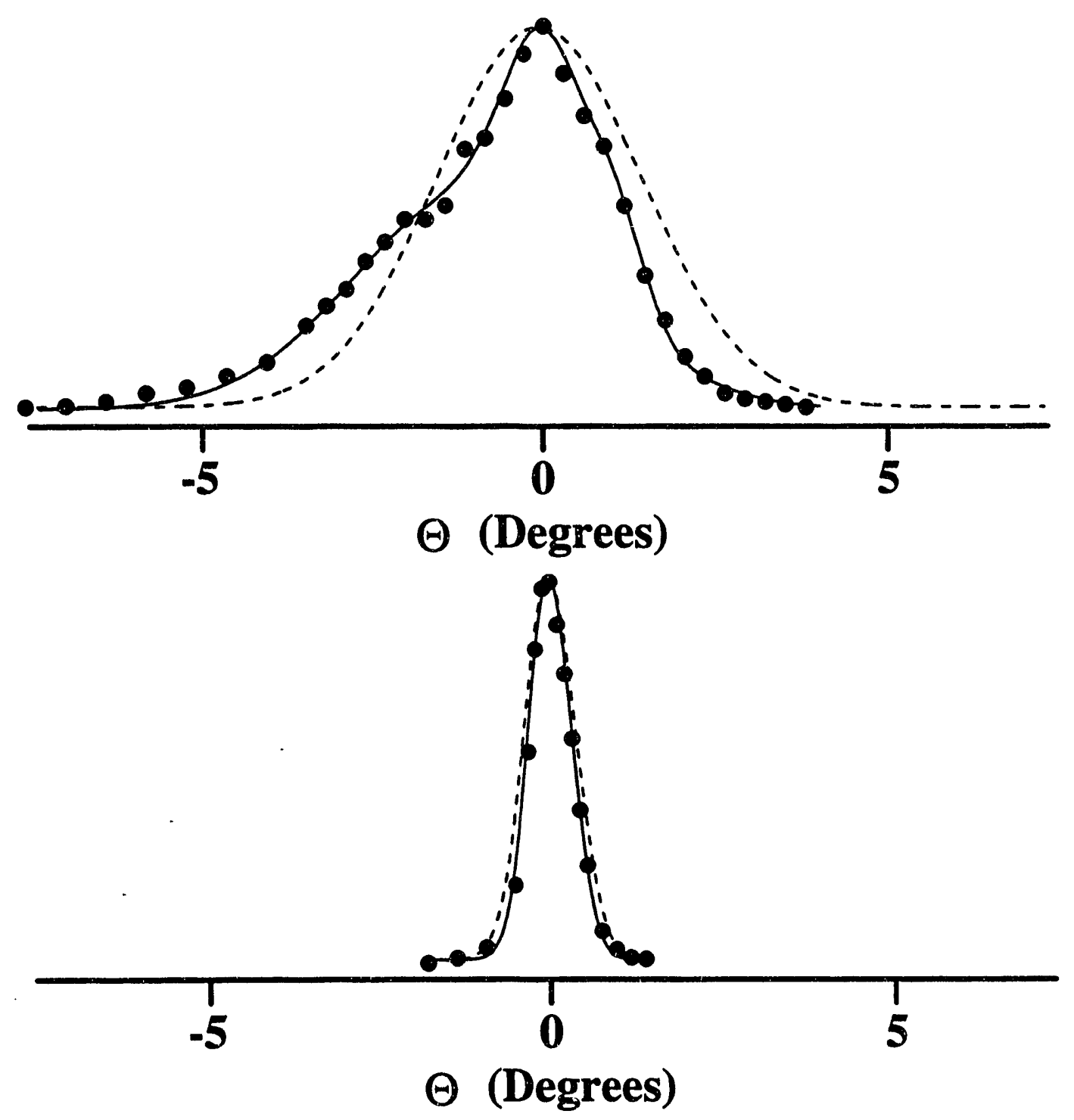

Figure 4.6: Mosaic spread of the orientation of the graphene layers in $\mathrm{Cs}(\mathrm{THF})_{1.3} \mathrm{C}_{24}$ (top) and $\mathrm{K}(\mathrm{THF})_{2.5} \mathrm{C}_{24}$ (bottom) as measured by $\mathrm{X}$-ray diffraction. With the detector set to verify the Bragg condition corresponding to the (002) reflection, the intensity as a function of sample orientation is a direct measure of the orientational distribution of the graphene layers in the sample. The solid circles represent experimental points, the solid lines represent Gaussian fits to the experimental data, and the dashed lines are the distributions used in the simulations of the experimental NMR spectra. 
mode originally postulated for cyclopentane [71] and subsequently for other fivemembered ring molecules [73]. Such ring systems are usually not planar, but puckered. In the model by Kilpatrick, Pitzer, and Spitzer [71], the distances of the ring atoms from the reference $x-y$ plane are given by :

$$
z(i)=\sqrt{\frac{2}{5}} q \cos \left(\frac{4 \pi}{5} i+\phi\right)
$$

where $i$ ranges from 0 to 4 and labels the ring atoms, starting with the oxygen atom, and $q$ and $\phi$ are the amplitude and phase of the puckering, respectively. Note that our definition of $\phi$ is different from that of Kilpatrick et al. [71]. In their definition $\phi$ ranges from $0^{\circ}$ to $180^{\circ}$ and in ours it ranges from $0^{\circ}$ to $360^{\circ}$. Kilpatrick's model was improved by Geise et al. [74] to minimize the variations in $\mathrm{C}-\mathrm{C}$ and $\mathrm{C}-\mathrm{O}$ bond lengths with changes in phase and amplitude of the puckering. They start with a planar reference compound defined by the spoke length $\mathrm{S}_{0}$ and the angles $\delta$ and $\epsilon$ as shown in Figure 4.7. The distances of the ring atoms from the $x-y$ plane in the puckered molecule are given by Equation 4.1 with the additional constraint that

$$
S(i)=S_{0}\left(1-k z^{2}(i)\right)
$$

where $S(i)$ is the spoke length in the puckered compound and $k$ is a constant equal to $0.65 \AA^{-2}$. Thus, using Equations 4.1 and 4.2 and the values of $S_{0}, \delta$, and $\epsilon$ of Geise, et al. [74], it is possible to calculate the coordinates of the oxygen and carbon atoms in THF as a function of the pseudorotation parameters $q$ and $\phi$. Several conformations of THF as a function of $\phi$ are shown in Figure 4.8 for a fixed value of $q$.

The proton coordinates for THF are calculated by assuming a fixed C-H bond length of $1.115 \AA[74]$ and an $\mathrm{H}-\mathrm{C}-\mathrm{H}$ angle of $109.47^{\circ}$. Each H-C-H plane is perpendicular to the plane defined by the carbon atom and its two nearest heavy atom 


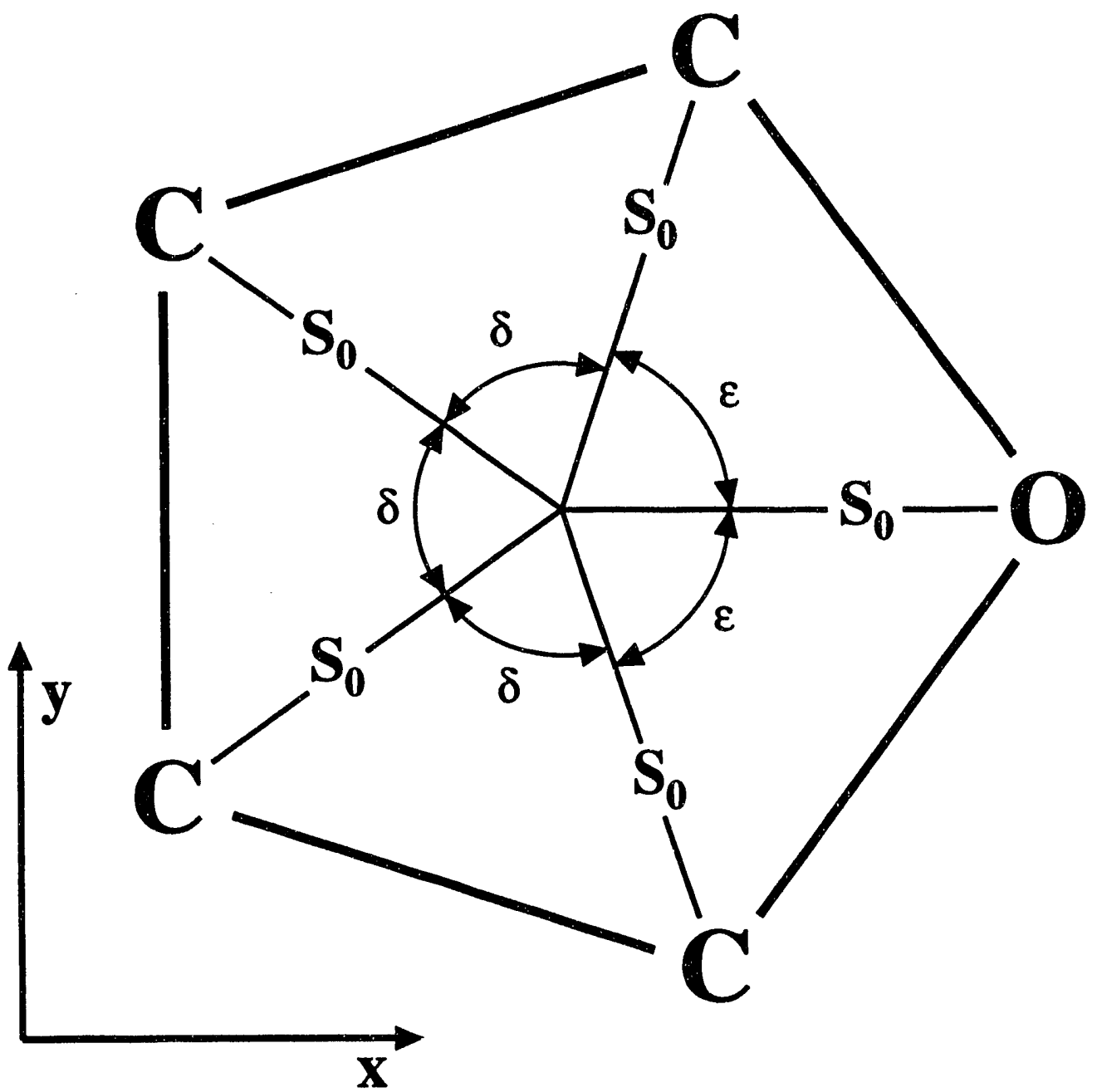

Figure 4.7: The planar reference compound for THF showing the $\mathrm{x}$ and $\mathrm{y}$ coordinates of the heavy atoms in THF. The positions of the atoms in the $x-y$ plane are defined by the angles $\delta$ and $\epsilon$ and by the spoke length $S_{0}$. 


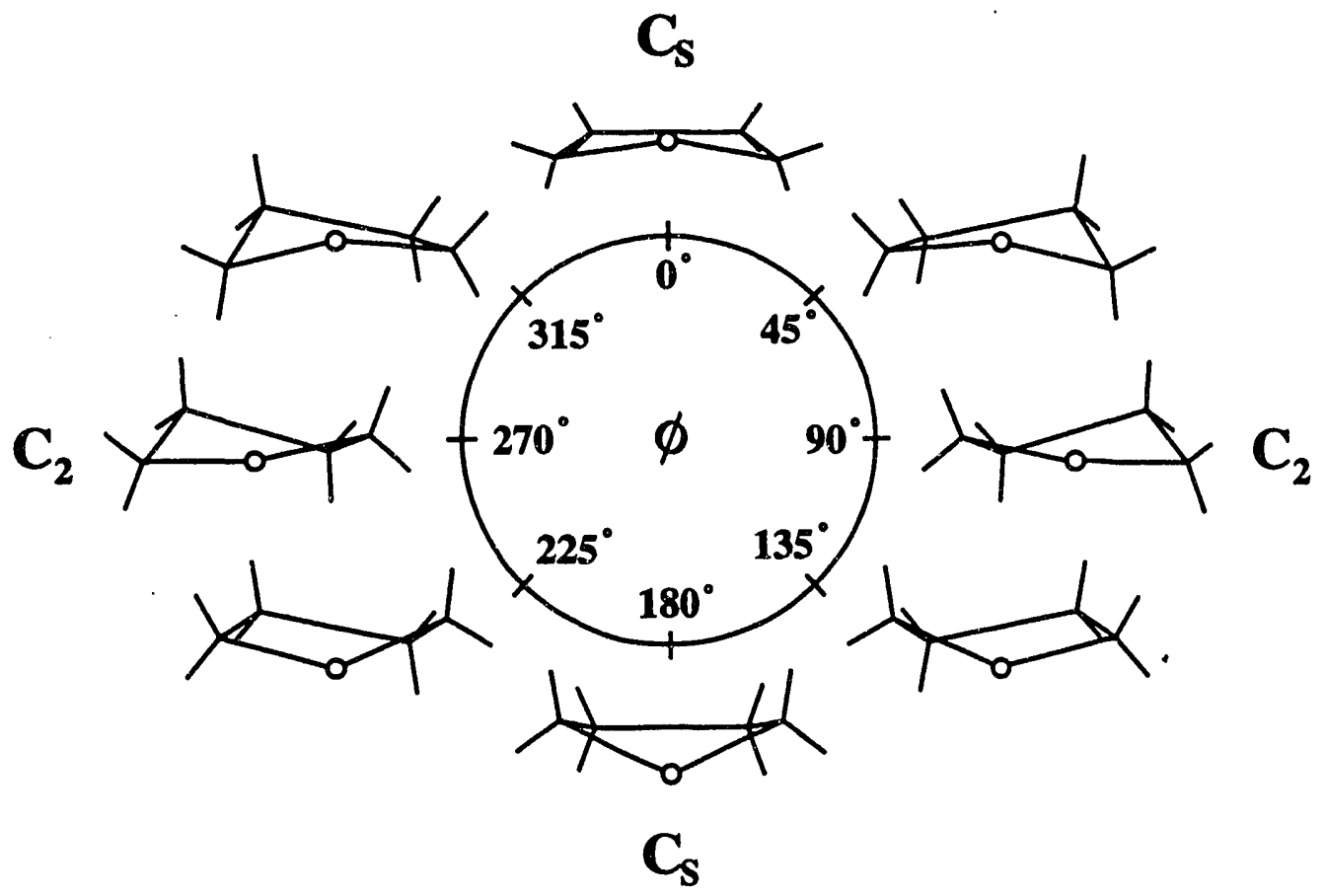

Figure 4.8: Pseudorotation of THF as a function of the pseudorotation phase $\phi$. 
neighbors; the intersection of both planes bisects the H-C-H angle. The geometrical parameters used in the simulations are summarized in Table 4.1.

Table 4.1: Geometrical parameters for THF

\begin{tabular}{|c|c|}
\hline$\delta$ & $74.52^{\circ}$ \\
$\epsilon$ & $68.22^{\circ}$ \\
$S_{0}$ & $1.277 \AA$ \\
C-H Bond & $1.115 \AA$ \\
H-C-H Angle & $109.47^{\circ}$ \\
\hline
\end{tabular}

To parameterize the potential for pseudorotation of THF, previous studies [75, 76,77 ] have used a potential of the form :

$$
V(\phi)=V_{2} \cos (2 \phi)+V_{4} \cos (4 \phi)
$$

where $V_{2}$ and $V_{4}$ are constants and $\phi$ is the phase of the pseudorotation. Greenhouse, et al. [76] and Engerholm, et al. [77] found values around $V_{2}=0.16 \mathrm{~kJ} / \mathrm{mol}$ and $V_{4}=$ $0.24 \mathrm{~kJ} / \mathrm{mol}$ for gaseous THF, corresponding to slightly hindered pseudorotation. Esteban, et al. [75] were unable to determine unique values for $V_{2}$ and $V_{4}$ for THF dissolved in a nematic liquid crystal, but concluded that their data was most consistent with nearly free pseudorotation.

In addition to dipole couplings, the chemical shifts of the protons in THF could play a role in determining the shape of the NMR spectra. The measured spectra are almost symmetric at all sample orientations, showing that the chemical shift difference between the $\alpha$ - and $\beta$ - protons of THF is small compared to the dipole couplings. For the simulations here a chemical shift difference of $200 \mathrm{~Hz}(2 \mathrm{ppm})$ was used. This is approximately the same as the difference in the measured isotropic chemical shift values $[78,79]$.

An attempt was made to simulate the NMR spectra of THF in the K/THF 
and Cs/THF GICs using the geometrical parameters in Table 4.1 and adjusting the pseudorotation amplitude $q$, the coefficients $V_{2}$ and $V_{4}$ of the pseudorotation potential, and the orientation of the THF molecule within the graphene layers. The simulations were done by density matrix calculations like in Equation 1.53 for an eight-spin THF molecule. The Hamiltonian is first constructed by averaging over all conformations and orientations sampled by the molecule. Then it is diagonalized, and Equation 1.59 is used to calculate the NMR spectra.

\subsection{Results}

\subsection{1 $\mathrm{Cs}(\mathrm{THF})_{1.3} \mathrm{C}_{24}$}

Previous studies of saturated Cs/THF GICs [57, 65] concluded that due to steric constraints imposed by the small interlayer spacing $\left(I_{c}\right)$ of $7.1 \AA$, the THF molecules must have their mean planes parallel to the graphene layers. It was also proposed that at room temperature the THF molecules are rotating about an axis perpendicular to the graphene planes. Both of these conclusions are supported by the data and simulations in this chapter. The width of the spectra in Figure 4.4 as a function of $\theta$, the angle between the normal to the graphene layers and the external magnetic field, retain roughly the same shape, except scaled by $3 \cos ^{2} \theta-1$, and collapse to a single line at the magic angle. This can only occur if all THF molecules are rapidly spinning about one common axis - the axis perpendicular to the graphene layers. A rapid rotation of complexes consisting of one cation and a few THF molecules is also possible. The THF molecules must also have their mean planes approximately parallel to the graphene layers, as this is the only orientation 
for which the simulations give spectra with features which extend past $\pm 40 \mathrm{kHz}$ as in the experimental spectra. Any other orientation gives simulated spectra which are too narrow.

With the mean plane of THF parallel to the graphene layers and rotation about an axis perpendicular to the graphene layers, simulation of the $\theta=0^{\circ}$ $\mathrm{Cs}(\mathrm{THF})_{1.3} \mathrm{C}_{24}$ spectrum was optimized with respect to the parameters $q$ (the ring puckering amplitude) and $V_{2}$ and $V_{4}$ (the constants in the pseudorotation potential in Equation 4.3). The best fit to the experimental data was obtained with $q=0.30 \pm 0.01 \AA, V_{2}=-15 \pm 5 \mathrm{~kJ} / \mathrm{mol}$, and $V_{4}=3 \pm 2 \mathrm{~kJ} / \mathrm{mol}$ and is shown in Figures 4.9 and 4.10. The value of $q$ is somewhat less than that found for gaseous THF of $0.38-0.44 \AA[72,74,80]$ and that found for THF in a liquid crystal of 0.39 $\AA$ [75]. The values found for $V_{2}$ and $V_{4}$ correspond not to hindered pseudorotation, but instead to interconversion between only two conformations at $\phi=0^{\circ}$ and $180^{\circ}$ with a barrier of $30 \mathrm{~kJ} / \mathrm{mol}$ between them. The possibiiity that THF is fixed in one conformation, $\phi=0^{\circ}$, was also considered. With this assumption, it was found that the experimental spectra could be most accurately simulated with $q=0.32 \pm 0.01$ $\AA$, as shown in Figures 4.11 and 4.12 .

The differences in the conformational mobility and puckering amplitude between THF in a gas or liquid crystal and THF intercalated into the layers of graphite are most likely a result of the constrained environment imposed by the graphene layers. The graphite matrix and the interaction with the cations seem to deform the THF molecules slightly from their equilibrium conformation, as well as to prevent them from pseudorotating. 


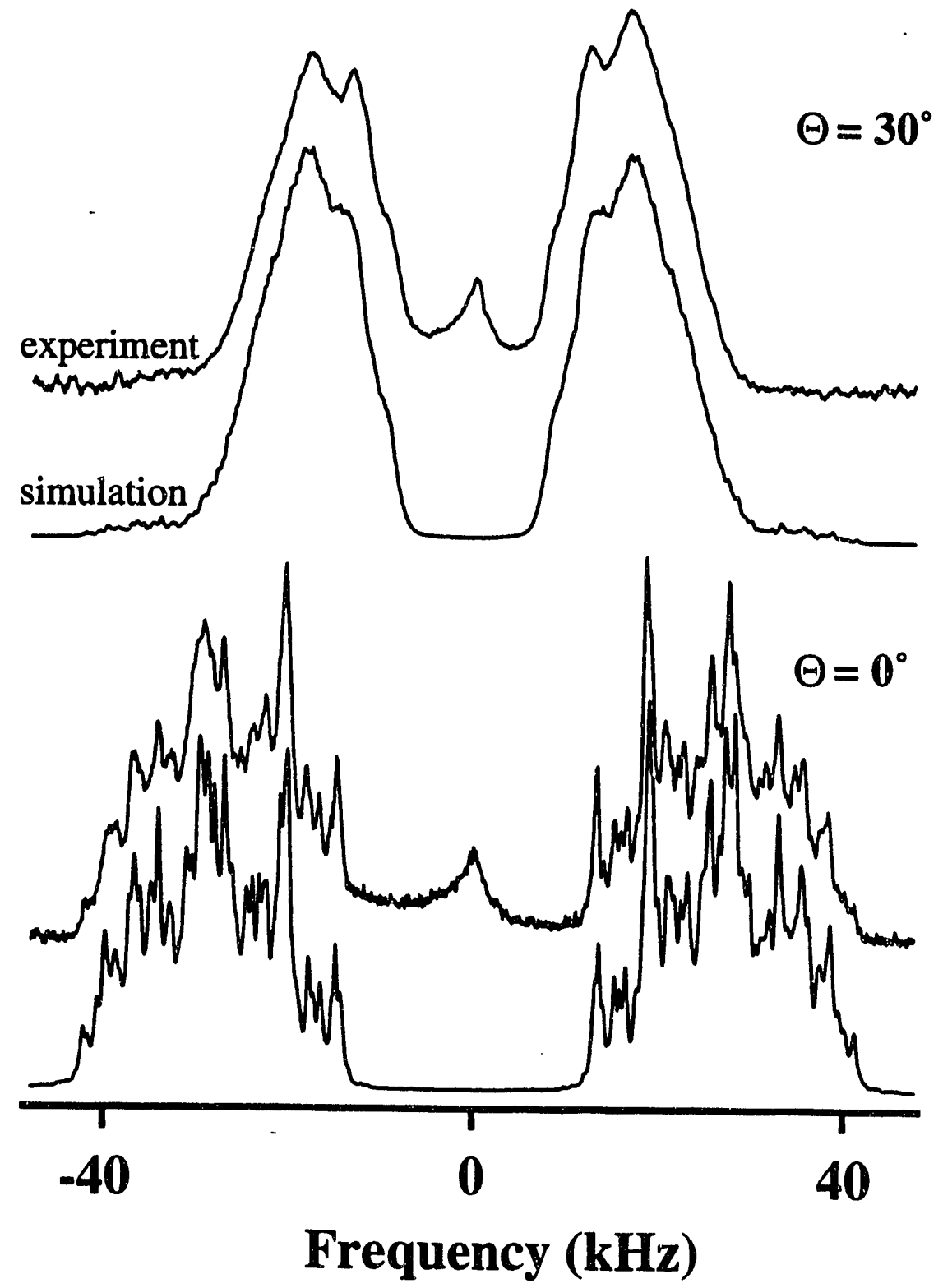

Figure 4.9: Simulation of $\mathrm{Cs}(\mathrm{THF})_{1.3} \mathrm{C}_{24}$ for $\Theta$, the angle between the external magnetic field and the normal to the graphene layers, having the values $0^{\circ}$ and $30^{\circ}$. The simulations were carried out with the mean planes of the THF molecules parallel to the graphene layers and the molecules rotating about an axis perpendicular to the layers. The parameters $q=0.30 \AA, V_{2}=-15 \mathrm{~kJ} / \mathrm{mol}$, $V_{4}=3 \mathrm{~kJ} / \mathrm{mol}$, and line broadening of $200 \mathrm{~Hz}$ were used, and appropriately weighted spectra of different orientations were added together to simulate the mosaic spread of the layers in Figure 4.6. 


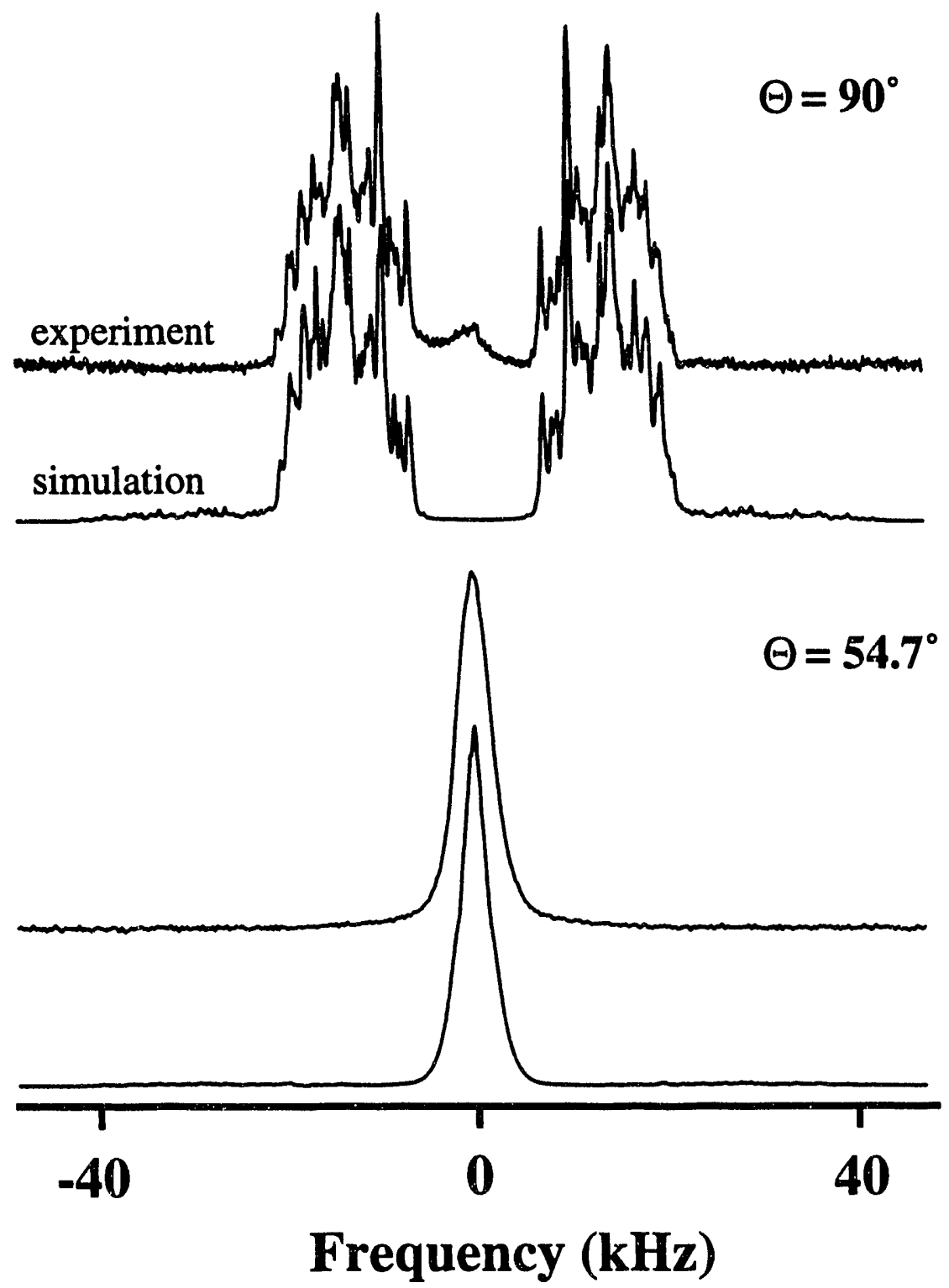

Figure 4.10: Simulation of $\mathrm{Cs}(\mathrm{THF})_{1.3} \mathrm{C}_{24}$ for $\Theta$, the angle between the external magnetic field and the normal to the graphene layers, having the values $54.7^{\circ}$ and $90^{\circ}$. The simulations were carried out with the mean planes of the THF molecules parallel to the graphene layers and the molecules rotating about an axis perpendicular to the layers. The parameters $q=0.30 \AA, V_{2}=$ $-15 \mathrm{~kJ} / \mathrm{mol}, V_{4}=3 \mathrm{~kJ} / \mathrm{mol}$, and line broadening of $200 \mathrm{~Hz}$ were used, and appropriately weighted spectra of different orientations were added together to simulate the mosaic spread of the layers in Figure 4.6. 


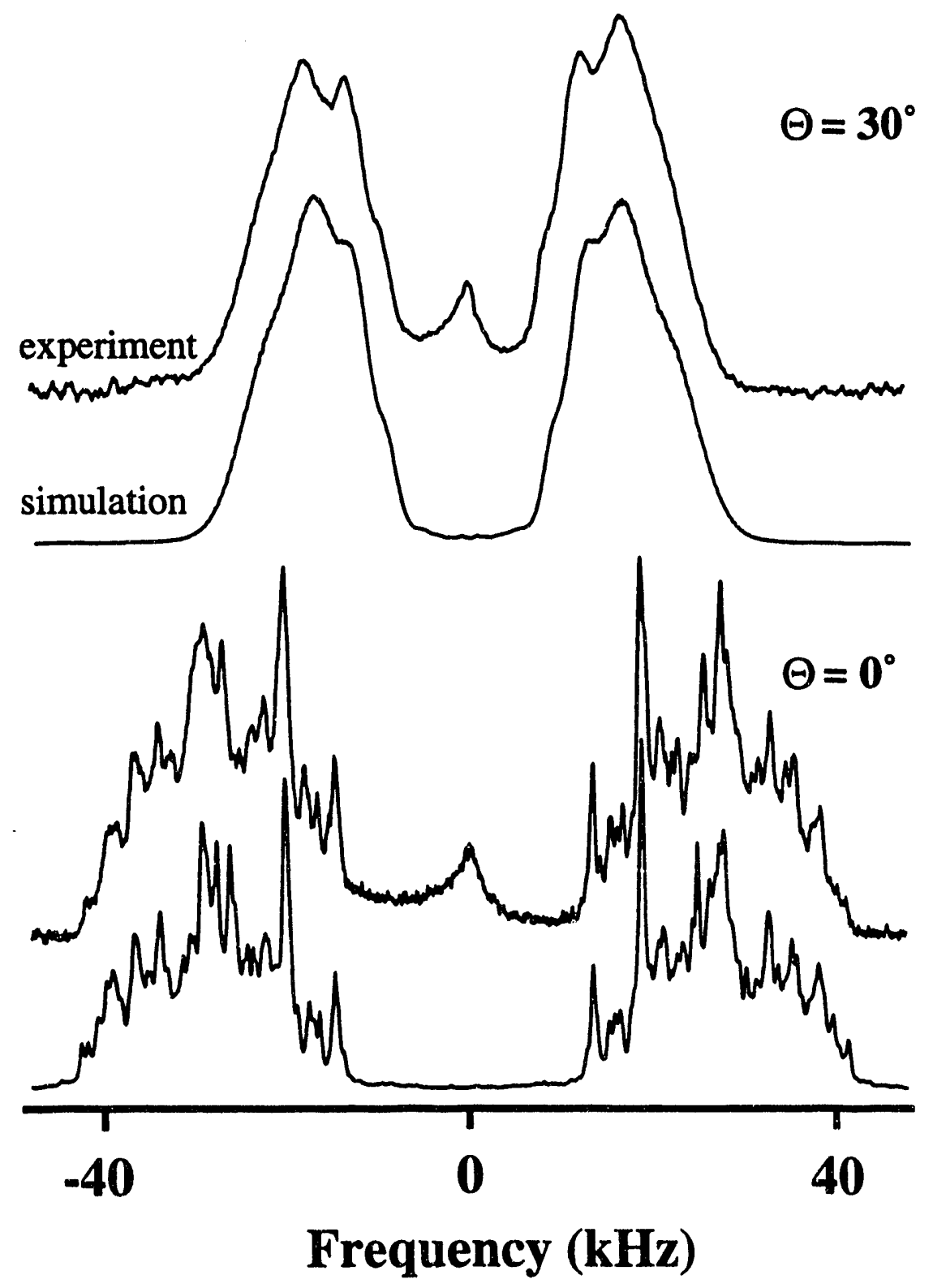

Figure 4.11: Simulation of the spectra $\mathrm{Cs}(\mathrm{THF})_{1.3} \mathrm{C}_{24}$ as in Figure 4.9 except with only one conformation, $\phi=0^{\circ}$, and with $q=0.32 \AA$. 


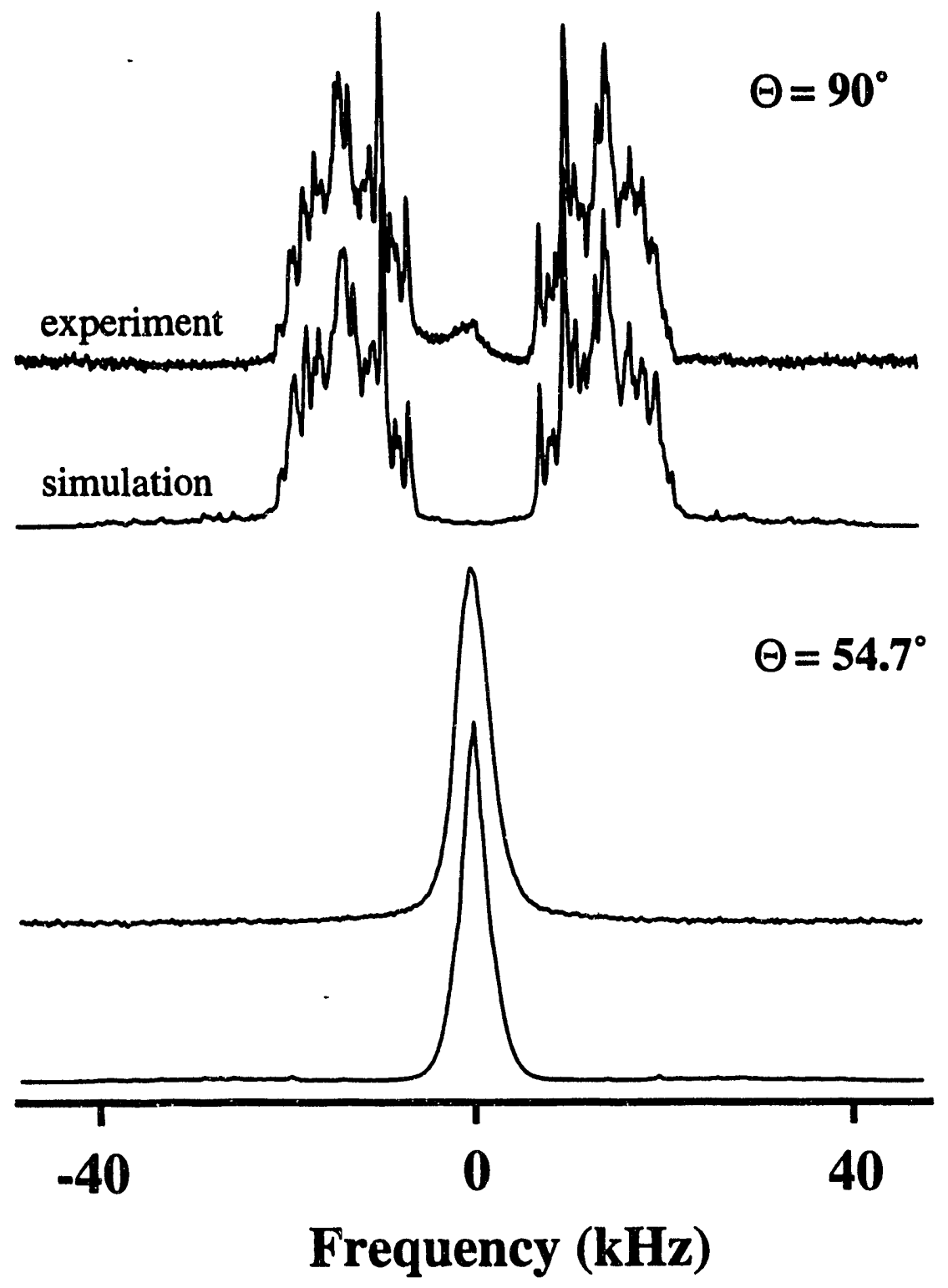

Figure 4.12: Simulation of the spectra $\mathrm{Cs}(\mathrm{THF})_{1.3} \mathrm{C}_{24}$ as in Figure 4.10 except with only one conformation, $\phi=0^{\circ}$, and with $q=0.32 \AA$. 


\subsection{2 $\mathrm{K}(\mathrm{THF})_{2.5} \mathrm{C}_{24}$}

The spectra of $\mathrm{K}(\mathrm{THF})_{2.5} \mathrm{C}_{24}$ in Figure 4.5 are quite different from those of the Cs/THF GIC in Figure 4.4, indicating that the orientation and motion of THF in the K/THF GIC are quite different from those in the Cs compound. Previous studies of saturated K/THF GICs $[58,81]$ have concluded from the presence of more THF per alkali atom and the larger interlayer spacing of $8.9 \AA$ that the mean planes of the THF molecules are approximately perpendicular to the graphene layers. It was also concluded that the THF molecules are oriented such that their oxygen atoms are near a graphene layer and that the THF molecules or $\mathrm{K}^{+} / \mathrm{THF}$ complexes are spinning about an axis perpendicular to the graphene layers. Our initial simulations showed that the THF molecules could not have their mean planes exactly perpendicular to the graphene layers, but must have some orientation between perpendicular and parallel. Varying the orientation of the THF molecules in addition to their conformation would introduce two more adjustable parameters. This leads to a large number of adjustable parameters for the simulations, so a few simplifying assumptions were made for the further simulations. Since the lineshape appears to be more sensitive to changes in the orientation than to the details of the molecular conformation, the values of $q, V_{2}$, and $V_{4}$ were no longer varied, but rather taken from the best simulations of the $\mathrm{Cs}(\mathrm{THF})_{1.3} \mathrm{C}_{24}$ compound. Only the reorientational motions of the THF molecules were varied. Like in the Cs/THF GIC, the THF molecules must be rotating about an axis perpendicular to the graphene planes, since the overall width of the spectra in Figure 4.5 as a function of $\Theta$ scales as $3 \cos ^{2} \theta-1$.

The orientation of a THF molecule with respect to the graphene layers is defined 
by the two Euler angles $\alpha$ and $\beta$ which involve counterclockwise rotations of the reference orientation shown in Figure 4.7 about space-fixed axes with the layer normal along the $\mathrm{z}$ axis. The first rotation is by the angle $\alpha$ about the $\mathrm{z}$ axis, followed by a rotation of $\beta$ about the $y$ axis. The best result for the case of two conformations of THF ( $q=0.30, V_{2}=-15 \mathrm{~kJ} / \mathrm{mol}$, and $\left.V_{4}=3 \mathrm{~kJ} / \mathrm{mol}\right)$ was obtained by averaging over orientations of THF defined by the Euler angles $\alpha=40^{\circ}$ to $120^{\circ}$ and $\beta=71^{\circ}$ and is shown in Figures 4.13 and 4.14. In this simulation the THF molecules have their mean planes tilted slightly away from perpendicular to the graphene layers, and oscillate about an axis perpendicular to the rings' mean planes, moving the oxygen atoms through a range of angles which keep it away from the graphene layers. The simulations with only one conformation $\left(\phi=0^{\circ}\right.$ and $q=0.32 \AA$ ) gave a best fit to the experimental spectra for THF oriented at Euler angles of $\alpha=22^{\circ}$ and $\beta=55^{\circ}$, shown in Figures 4.15 and 4.16. In this case the mean plane of THF is tilted further away from the layer normal, with the oxygen atoms oriented approximately towards the layers. These results show that the THF molecules can not have their mean planes perpendicular to the graphene layers, but most likely have their mean planes between $50^{\circ}$ and $75^{\circ}$ from parallel to the graphene layers. Additional motions, besides the rotation about an axis perpendicular to the graphene layers, are possible, but cannot be discerned based on our simulations.

\subsection{Conclusions}

Proton NMR spectra of $\mathrm{Cs}(\mathrm{THF})_{1.3} \mathrm{C}_{24}$ and $\mathrm{K}(\mathrm{THF})_{2.5} \mathrm{C}_{24}$ have been analyzed to determine the orientations and motions of the THF molecules in these compounds 


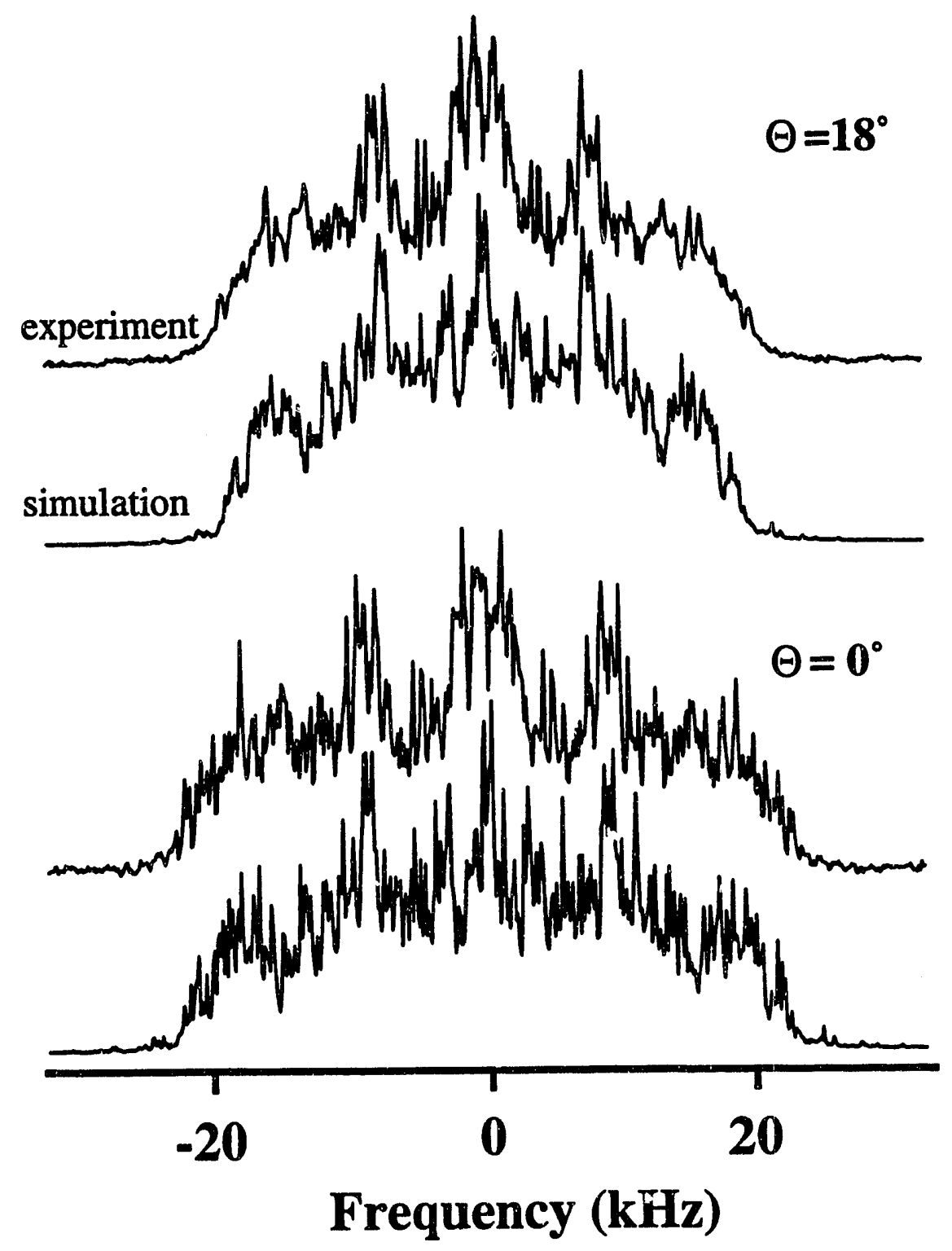

Figure 4.13: Simulation of $\mathrm{K}(\mathrm{THF})_{2.5} \mathrm{C}_{24}$ for $\Theta$, the angle between the external magnetic field and the normal to the graphene layers, having the values $0^{\circ}$ and $18^{\circ}$. The simulations were carried out by averaging over orientations of the THF molecules with respect to the graphene layers defined by the Euler angles $\alpha=40^{\circ}$ through $120^{\circ}$ and $\beta=71^{\circ}$ and the molecules rotating about an axis perpendicular to the layers. The parameters $q=0.30 \AA, V_{2}=-15 \mathrm{~kJ} / \mathrm{mol}, V_{4}$ $=3 \mathrm{~kJ} / \mathrm{mol}$, and line broadening of $100 \mathrm{~Hz}$ were used. 


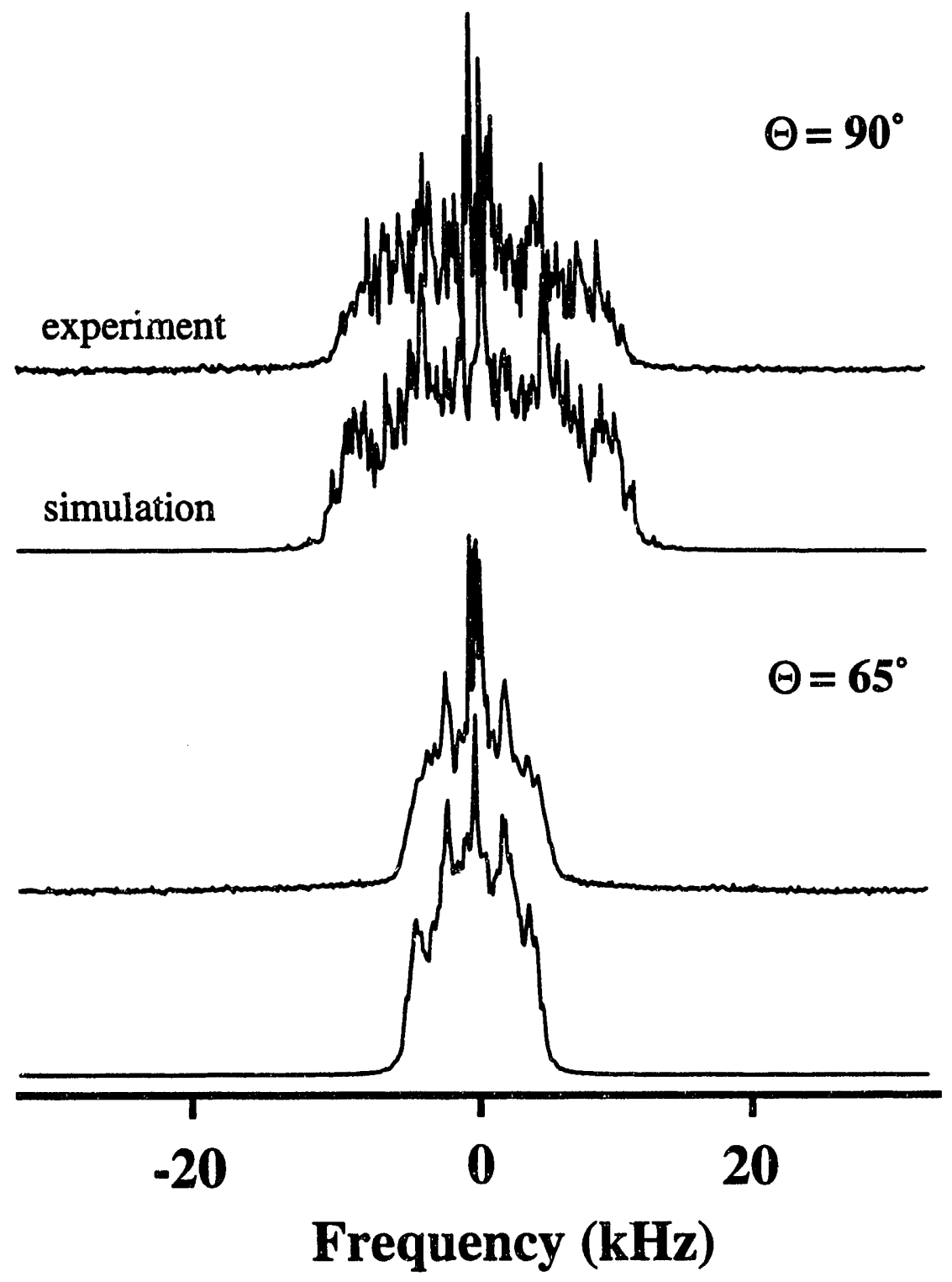

Figure 4.14: Simulation of $\mathrm{K}(\mathrm{THF})_{2.5} \mathrm{C}_{24}$ for $\Theta$, the angle between the external magnetic field and the normal to the graphene layers, having the values $65^{\circ}$ and $90^{\circ}$. The simulations were carried out by averaging over orientations of the THF molecules with respect to the graphene layers defined by the Euler angles $\alpha=40^{\circ}$ through $120^{\circ}$ and $\beta=71^{\circ}$ and the molecules rotating about an axis perpendicular to the layers. The parameters $q=0.30 \AA, V_{2}=-15$ $\mathrm{kJ} / \mathrm{mol}, V_{4}=3 \mathrm{~kJ} / \mathrm{mol}$, and line broadening of $100 \mathrm{~Hz}$ were used. 


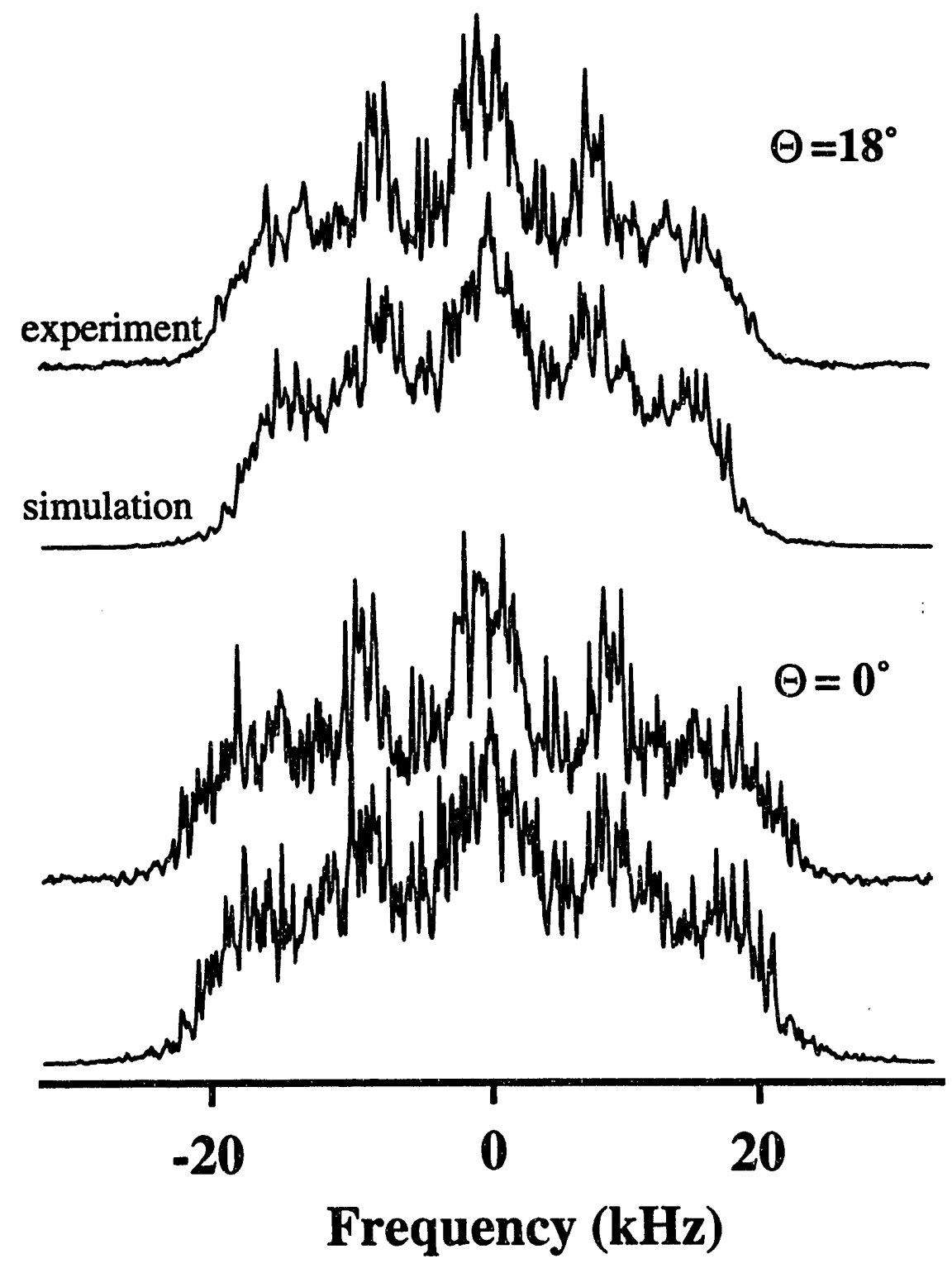

Figure 4.15: Simulation of the spectra $\mathrm{K}(\mathrm{THF})_{2.5} \mathrm{C}_{24}$ as in Figure 4.13 except with only one conformation, $\phi=0^{\circ}$, and with $q=0.32 \AA$. The orientation of the THF molecules with respect to the graphene planes is defined by the Euler angles $\alpha=22^{\circ}$ and $\beta=55^{\circ}$. 


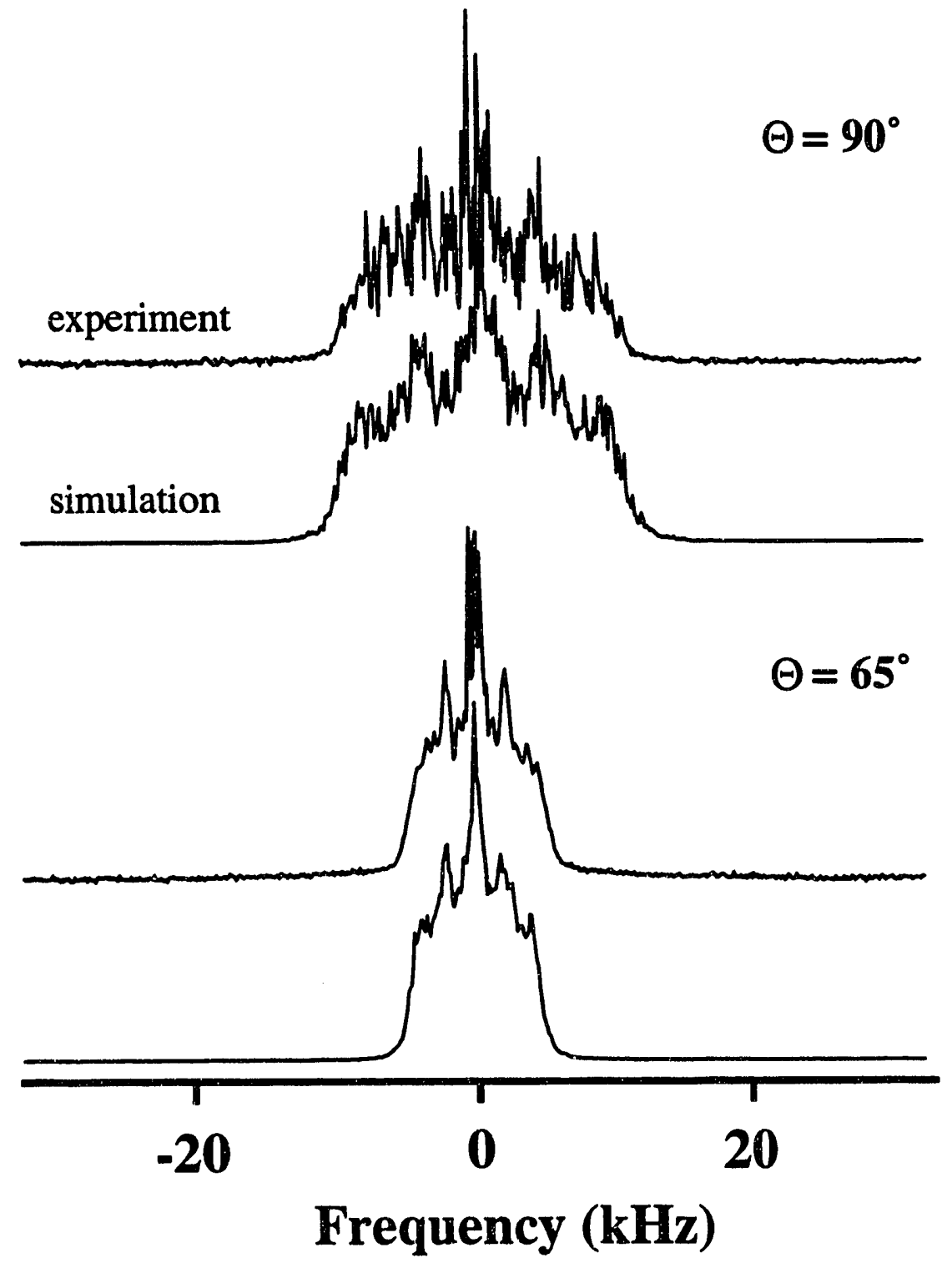

Figure 4.16: Simulation of the spectra $\mathrm{K}(\mathrm{THF}){ }_{2.5} \mathrm{C}_{24}$ as in Figure 4.14 except with only one conformation, $\phi=0^{\circ}$, and with $q=0.32 \AA$. The orientation of the THF molecules with respect to the graphene planes is defined by the Euler angles $\alpha=22^{\circ}$ and $\beta=55^{\circ}$. 
at 293K. Simulations of the NMR specra of Cs/THF GIC have confirmed previous conclusions that the THF molecules have their mean planes parallel to the graphene layers and are rotating about an axis perpendicular to the layers. Pseudorotation of THF in this compound is unlikely since a simulation of the spectrum of $\mathrm{Cs}(\mathrm{THF})_{1.3} \mathrm{C}_{24}$ with free pseudorotation, shown in Figure 4.17, differs significantly from the experimental spectrum. Simulations of the spectra of the K/THF GIC are not as conclusive, although they indicate that the THF molecules are oriented with their mean planes between $50^{\circ}$ and $75^{\circ}$ with respect the graphene layers, balanced between the forces from the graphene layers above and below and neighboring THF molecules and $\mathrm{K}^{+}$ions on the sides. The experimental data and simulations show that the THF molecules are rotating about an axis perpendicular to the graphene layers, as in the Cs compound, but that they cannot be freely rotating about an axis perpendicular to the mean planes of their rings. The question of pseudorotation of THF in the K compound cannot be answered. None of the simulations of this compound - with two conformations (Figures 4.13 and 4.14), with one conformation (Figure 4.15 and 4.16), and with free pseudorotation (Figure 4.17) - fits the experimental data well enough to rule out the others. Further studies using deuterium or multiple-quantum NMR could give more information on the nature of the motion occurring in these compounds [82]. 

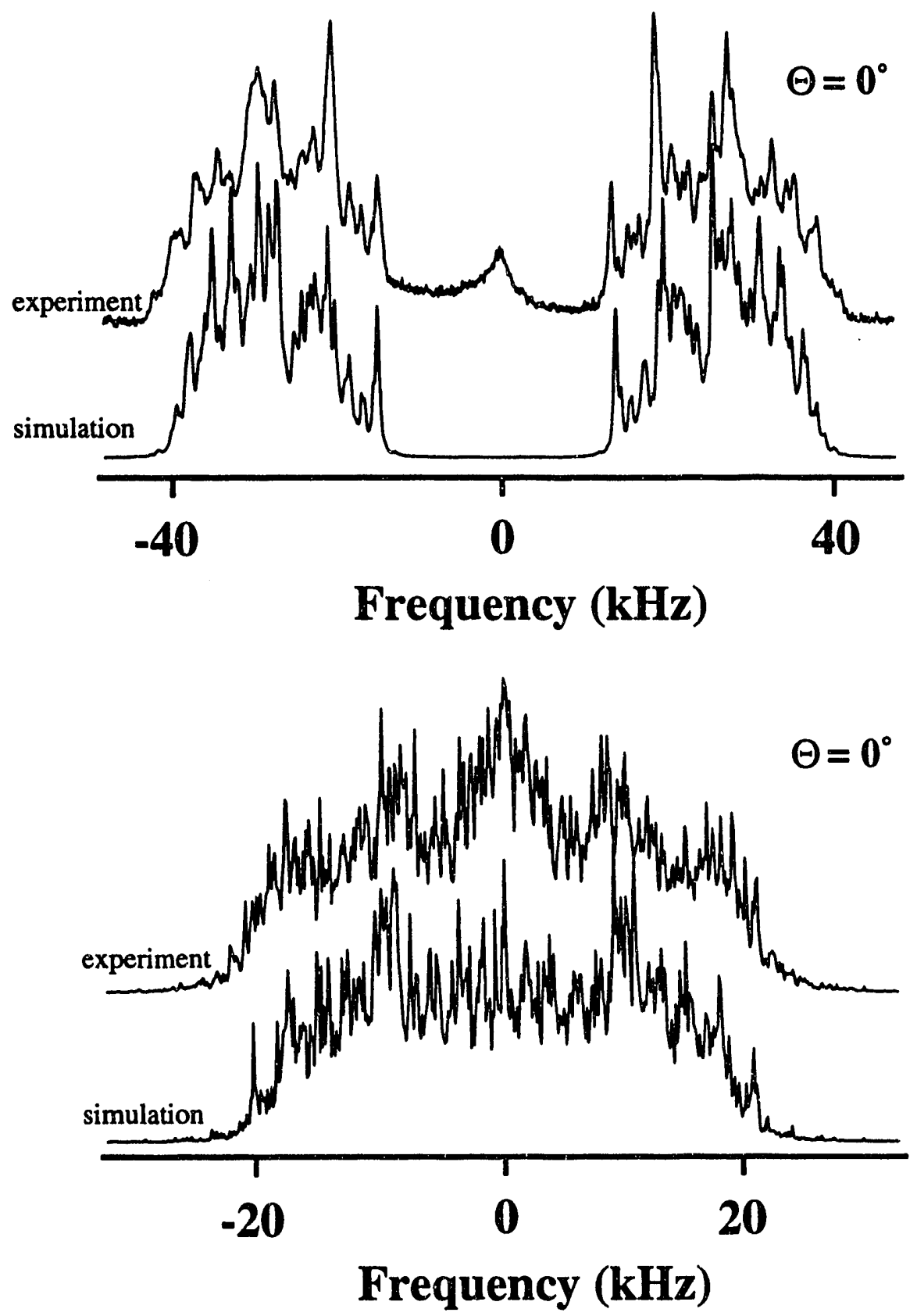

Figure 4.17: Simulation of Proton NMR Spectrum of $\mathrm{Cs}(\mathrm{THF})_{1.3} \mathrm{C}_{24}$ and $\mathrm{K}(\mathrm{THF})_{2.5} \mathrm{C}_{24}$ as in Figures 4.9 and 4.13 with free pseudorotation $\left(V_{2}=V_{4}=\right.$ 0). 


\section{Chapter 5}

\section{Tecmag Pulse Programmer Control Software}

This chapter describes the software used to control a Tecmag, Inc. [83] pulse programmer/signal acquisition unit with a DEC VAXStation II or MicroVAX II computer. The software provided by Tecmag, Inc. proved to be inadequate for control and operation of the unit, so the TECMAG program to be described in this chapter was written. The software and Tecmag PULSkit/SAkit unit is presently on the Delta spectrometer, which is described in previous theses [37, 84].

\subsection{Hardware}

The Tecmag PULSkit/SAkit unit is designed to provide TTL pulses at a rate of up to $5 \mathrm{MHz}$ and to acquire data on two channels at a rate of up to $1 \mathrm{MHz}$. It requires an analog $10 \mathrm{MHz}$ clock signal at approximately $1 \mathrm{~V}_{p p}$ (though it may not function properly if the clock signal is $<1 \mathrm{~V}_{p p}$ or if the clock signal is noisy). This clock is 
usually supplied by the PTS frequency synthesizer used on the spectrometer. The PULSkit and SAkit are enclosed in a single rack-mount box and each communicates with the VAXStation II running VMS through a 16-bit DR11-W parallel interface card from MDB Systems [85]. The two DR11-W's are known to the VMS operating systems as the devices XAA0: and XAB0:. XAA0: is the upper of the two DR11W's in the VAXStation II backplane and is connected to the SAkit (see Figure 5.1). Its bus address and vector address are octal 760240 and 300, respectively. XAB0: is connected to the PULSkit as shown in Figure 5.1, and has bus and vector addresses of octal 760250 and 304 , respectively.

\subsubsection{PULSkit}

The PULSkit pulse programmer is based on an AMD 2910 microsequencer, which clocks out 128-bit microcode words of duration 0.2 microseconds or more from its associated 2048 word microcode memory. Each 128-bit microcode word is an event of fixed duration, usually a pulse, delay, loop, or data acquisition step. An AMD 9513 chip is also present in the PULSkit and contains five 16-bit loop counters which can used to form loops in the microcode. A microcode word is shown in Figure 5.2. Starting with the highest number bit, each will now be described :

\section{Bits Description}

127-118 User-definable lines. No definition at present.

117 External trigger. If set, PULSkit stops until an external trigger pulse is received through the External Trigger line on the back of the Tecmag unit.

116-112 Loop counters. Each bit designates a loop counter to use with a loop instruction. Bit 112 corresponds to LC1.

111-108 Microsequencer instruction. Three instructions are used :

1. Continue - execute this instruction and continue to next one 
in sequence (hexadecimal code " $\mathrm{E}$ ").

2. Loop unconditional - loop to address specified in bits 106-96. (hexadecimal code "2").

3. Loop conditional - loop to address specified in bits 106-96 if the loop counter specified in bits 116-112 has a non-zero value. (hexadecimal code "3").

107 ATTN bit to send interrupt to VAXStation II.

106-96 Loop branch address. Address of microcode word to loop to.

95-94 User-definable lines. No definition at present

93 ADC bit to trigger analog-to-digital converters in the SAkit to start conversion.

92 SIG2 bit giving the sign of the data to be added to the imaginary buffer. 0 : "+" sign, 1 : "-" sign.

91 SIG1 bit giving the sign of the data to be added to the real buffer. 0 : "+" sign, 1 : "-" sign.

90 CHA bit to swap data input channels A and B. 0: A goes to real and $B$ goes to imaginary buffer. 1 : buffers swapped.

89-88 User-definable lines. No definition at present

$87 \quad$ PPG3 bit which resets the SAkit data memory address to zero.

86 CONV bit for data acquistion which causes a read of the current data word in the SAkit memory and adds it to the last output of the analog-to-digital converter.

85 EOC bit which completes the acquisition of a data point by writing it back to memory and incrementing the SAkit data memory address.

84-80 User-definable lines. No definition at present.

79-48 Two's complement time interval word in 0.1 microsecond increments. While instruction is executing, this is incremented each clock cycle until it equals zero, then the next instruction is executed.

47-35 User-definable lines. No definition at present.

34-25 Bits A10-A1. Auxiliary lines for the A (proton) channel of the spectrometer.

A8 is used for RF switch \#2 and must be high during a proton pulse.

A7 is used to toggle the PTS-500 frequency between two preprogrammed values.

A6 is used to toggle the digital phase shifter in and out of line.

A5 is used to reset the digital phase shifter.

A4 is used to increment the digital phase shifter.

24 ARF bit for RF switch \#1. Must be high during a proton pulse.

23-20 Gates for $\overline{\mathrm{Y}}, \mathrm{Y}, \overline{\mathrm{X}}$, and $\mathrm{X}$ pulses on the $\mathrm{A}$ (proton) channel. 
19-15 User-definable lines. No definition at present.

14-6 Auxiliary lines for the B channel.

B2 is used for ENI deblanking on the proton channel. For a proton pulse, this must be high for the delay preceding a pulse and during the pulse itself.

5 Receiver deblanking. This must be high during data acquistion.

4 BRF bit for the RF switch which must be high during a $B$ channel pulse.

3-0 Gates for $\bar{Y}, Y, \bar{X}$, and $\mathrm{X}$ pulses on the B channel.

The program used to control the Tecmag must load pulse programs composed of several microcode words, load values into the loop counters, and start and stop execution of these pulse programs. In Section 5.2 the TECMAG program which does this will be described.

\subsubsection{SAkit}

The SAkit contains two 12-bit analog-to-digital converters which can do a conversion in approximately 0.9 microseconds and $256 \mathrm{k}$ of 32 -bit words of memory for acquired data. It is connected to the VAXStation II computer through a DR11W parallel interface card. Acquisition of data is controlled by the PULSkit, and acquired data is transferred to the VAXStation II through the DR11-W.

\subsection{TECMAG Program}

\subsubsection{Overview}

The purpose of the TECMAG program is to control the Tecmag PULSkit/SAkit unit to perform an NMR experiment, collect data, and write it to disk. Processing and display of the data is done with separate programs (see Appendix A). The 


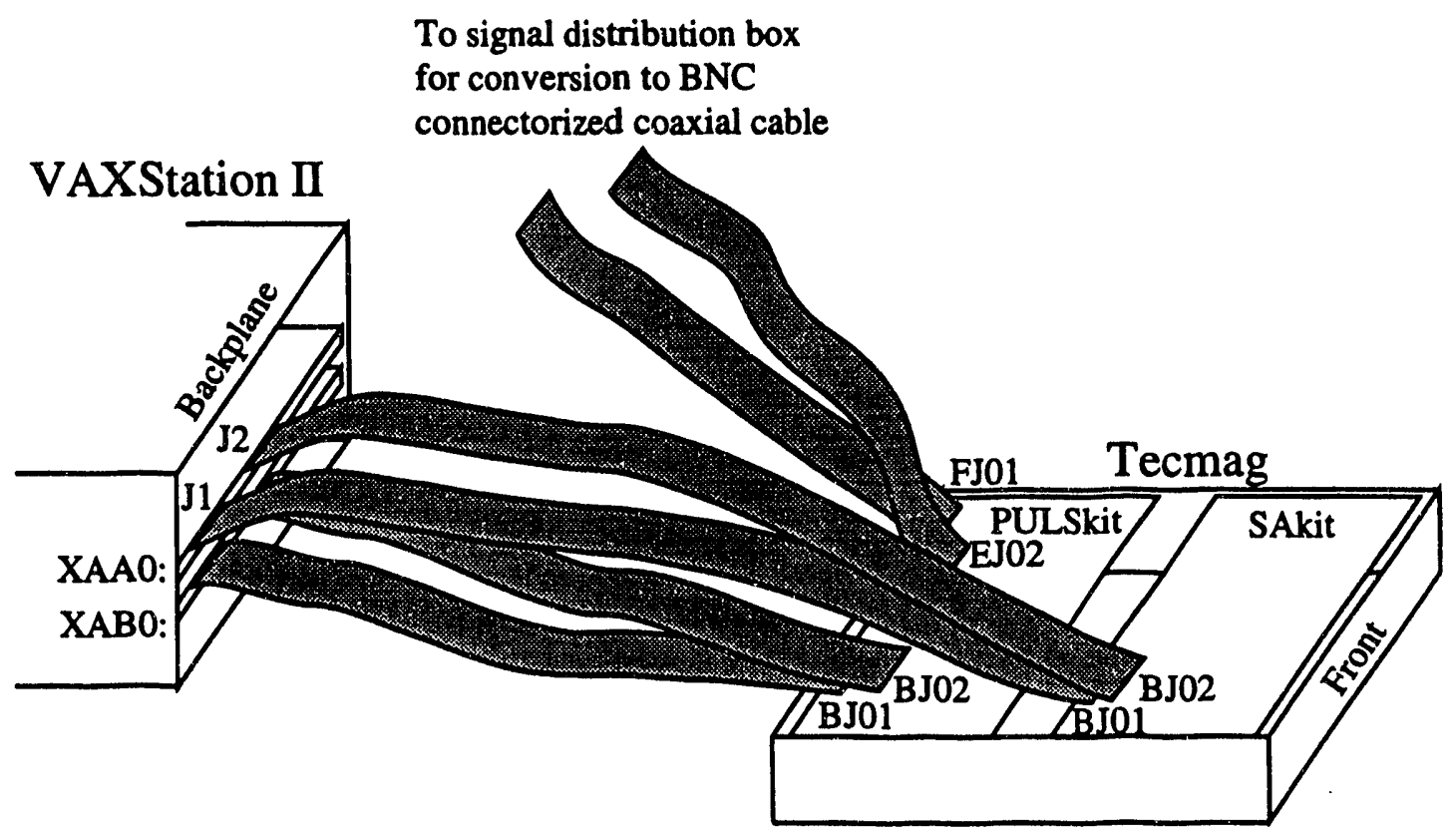

Figure 5.1: Connections between the VAXStation II and the Tecmag PULSkit and SAkit. Two 40-conductor ribbon cables connect the PULSkit and SAkit to DR11-W's in the VAXStation, as shown. The two other 40-conductor ribbon cables take output signals to a signal distribution box where the signals are buffered by TTL line drivers and redistributed on BNC connectorized coaxial cable. 

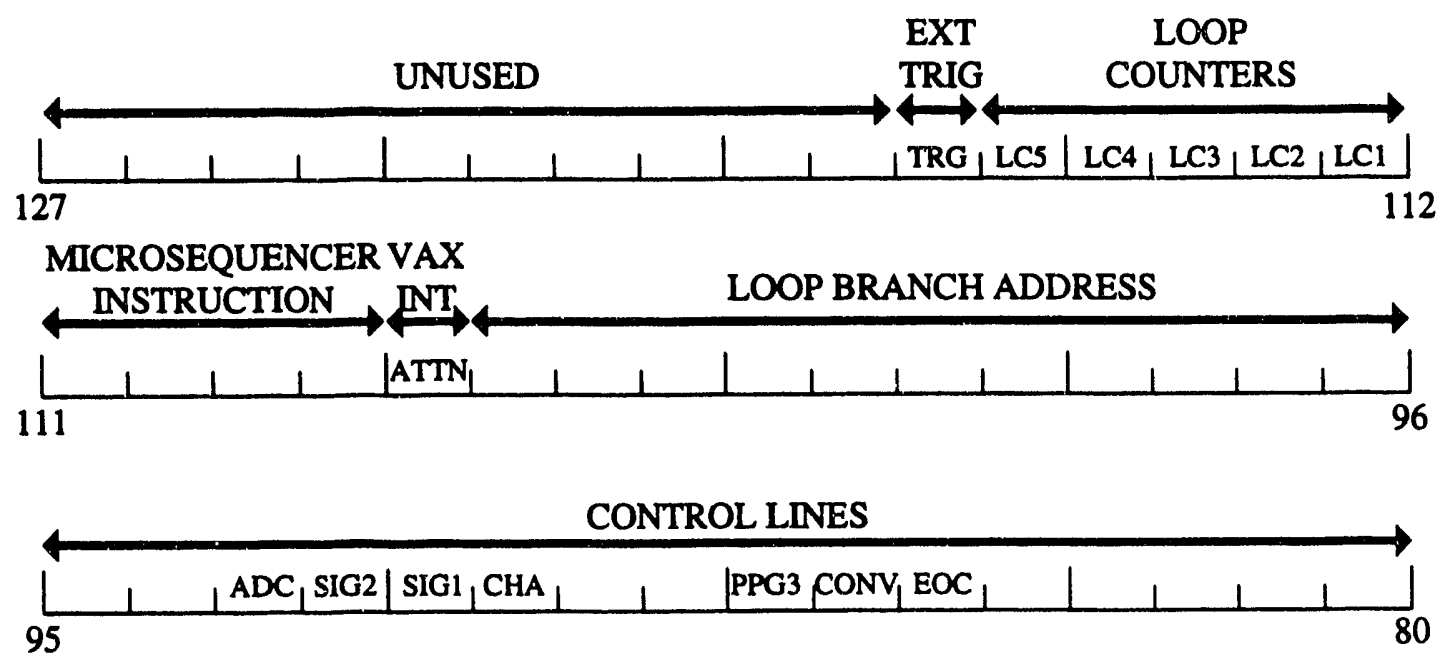

TIME INTERVAL (2'S COMPLEMENT - MSB)
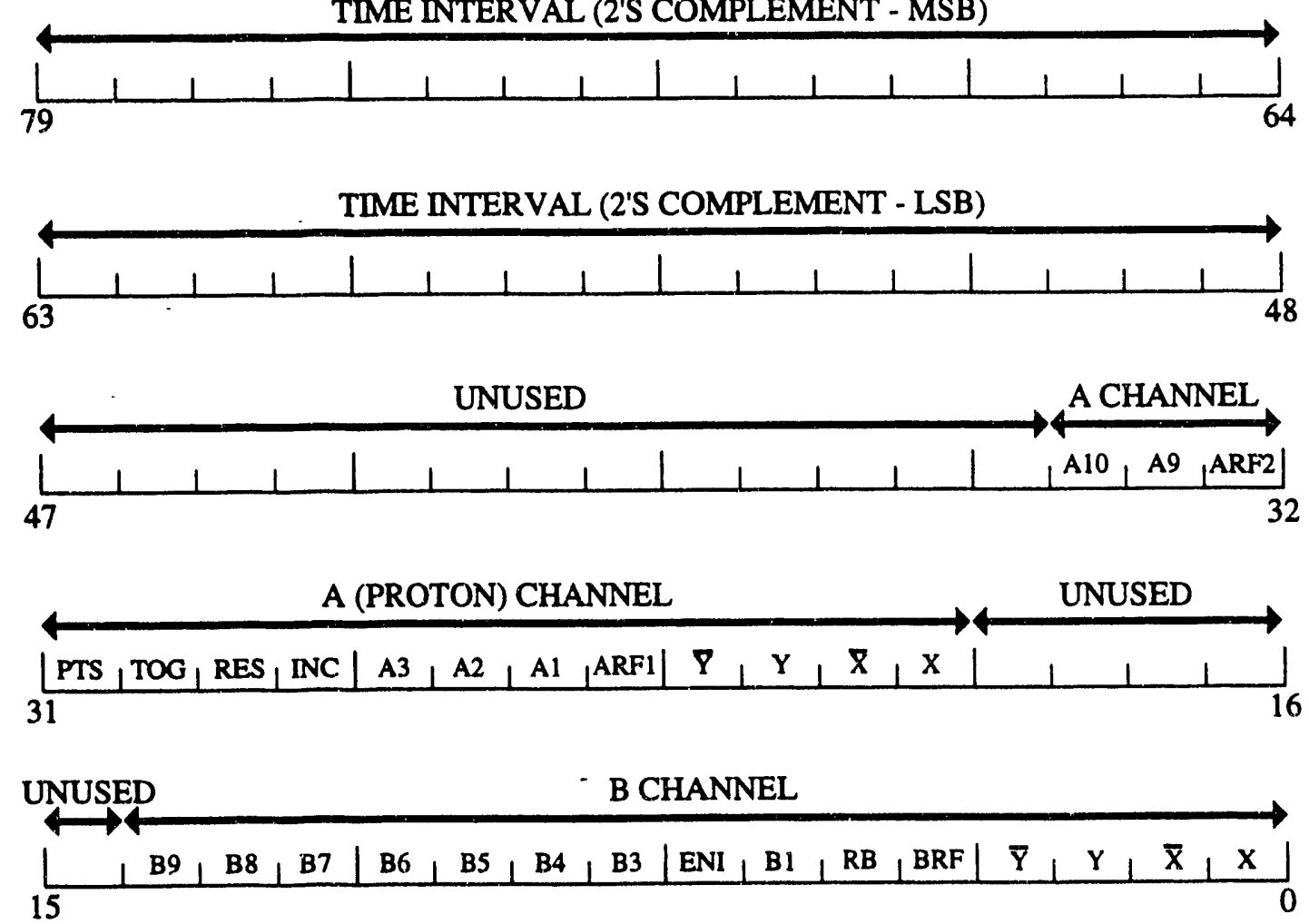

Figure 5.2: 128-bit microcode word for the PULSkit pulse programmer. A description of the lines is given in the text. 
TECMAG program reads in pulse programs which are written as lines of 128-bit microcode (written in hexadecimal), allows the user to change parameters such as time durations, phases of pulses, numbers of points, dwell time, and other experimental parameters, acquires complex data, and writes it to disk.

The program is built on a series of primitive commands that use the VMS system function "\$QIOW" to communicate through the DR11-W parallel interface cards with the PULSkit and SAkit. These primitives do the following :

- Attach DR11-W - assign a system I/O channel for device

- Reset DR11-W

- Detach DR11-W - deassign system I/O channel for device

- Initialize PULSkit and SAkit

- Set PULSkit to load mode

- Load PULSkit loop counters

- Load PULSkit memory with microcode

- Set PULSkit to execute mode

- Start and stop PULSkit

- Set SAkit to load mode

- Zero SAkit memory

- Set SAkit to execute mode

- Set SAkit to signal average mode

- Upload SAkit memory to VAXStation II

- Process interrupts generated when PULSkit sets ATTN bit high

On top of these primitives is a command interpreter which provides the user interface. The possible commands are listed in Table 5.1 with a brief description of each command. These will be discussed further in the examples in Sections 5.2.3 and 5.2.4. 
Table 5.1: TECMAG program commands

\begin{tabular}{|c|c|}
\hline$\overline{\mathrm{AT}}$ & - set acquisition time. \\
\hline $\mathrm{D} \chi$ & - set length of delay " $\chi$ " ( $\chi=0-9, \mathrm{~A}-\mathrm{H}, \mathrm{J}-\mathrm{V}, \mathrm{X}-\mathrm{Z})$. \\
\hline $\mathrm{DI} \chi$ & - set increment for delay " $\chi$ ". \\
\hline DW & - set dwell time. \\
\hline FS & - set when frequency shift bit (A7) is on. \\
\hline GO & - start pulse program without zeroing memory. \\
\hline HA & - halt pulse program after present scan. \\
\hline $\mathrm{LC} \chi$ & - set loop counter " $\chi$ " $(\chi=1,3,4,5)$. \\
\hline NA & - set number of averages per FID. \\
\hline NB & - set number of points acquired per FID. \\
\hline NF & - set number of FID's acquired in 2-D expt. \\
\hline PG & - read in pulse program (".mic" extension). \\
\hline$P \chi$ & - set length of pulse " $\chi$ " ( $\chi=0-9, \mathrm{~A}-\mathrm{F}, \mathrm{H}, \mathrm{J}-\mathrm{R}, \mathrm{T}-\mathrm{Z})$. \\
\hline $\mathrm{PI} \chi$ & - set increment for pulse " $\chi$ ". \\
\hline PSI & - set when phase shift increment bit (A4) is on. \\
\hline PSR & - set when phase shift reset bit (A5) is on. \\
\hline QU & - quit program. \\
\hline $\mathrm{RP}$ & - read parameter file (".acq" extension). \\
\hline ST & - stop pulse program immediately. \\
\hline SW & - set sweep width. \\
\hline UL & - upload data from SAkit and write to a file. \\
\hline WL & - write words to be loaded into loop counters. \\
\hline WM & - write present pulse program microcode. \\
\hline WPA & - write present acquisition parameters. \\
\hline WPH & - write present phase tables. \\
\hline $\mathrm{ZE}$ & - zero SAkit memory. \\
\hline ZG & - zero SAkit memory and start pulse program. \\
\hline
\end{tabular}




\subsubsection{Pulse Programming}

An example of a pulse program is given in Figure 5.3. This pulse program is for a standard one-dimensional proton experiment. Each line must be formatted exactly as in the example. A line starts with eight groups of four hexadecimal digits representing the microcode, with each group separated by a single space. This is followed by a 10 character line number (for convenience only, this is not used by the TECMAG program) and then a 10 character descriptor: The 10 character line number usually has 5 spaces followed by 4 characters which are hexadecimal digits starting with line 0 , followed by a single space. The descriptor begins with a two character mnemonic which has one of 4 forms :

- $\mathrm{D} \chi(\chi=0-9, \mathrm{~A}-\mathrm{H}, \mathrm{J}-\mathrm{V}, \mathrm{X}-\mathrm{Z})$ : the line is a delay

- $\mathrm{P} \chi(\chi=0-9, \mathrm{~A}-\mathrm{F}, \mathrm{H}, \mathrm{J}-\mathrm{R}, \mathrm{T}-\mathrm{Z})$ : the line is a pulse

- $\mathrm{A}$ : the line is a fixed delay during the acquisition sequence

- AL : the line is a variable delay during the acquisition sequence for the dwell time. This delay $\mathrm{f}$ us the fixed delay in lines with mnemonic " $\mathrm{A}$ " add up to the dwell time.

The mnemonic is followed by a space and then, if necessary, the fourth, fifth, and sixih characters of the mnemonic can be a number or letter $0-9$ or A-Z which specifies a phase table to use for a proton pulse, a pulse on the $B$ channel, or the receiver phase, respectively. The seventh character can take the value "I" or "R" for phase shifter incres.ent or reset.

A typical pu'se program like that in Figure 5.3 has four basic parts :

- An initial delay, the recycle delay (line 0)

- One or more pulses and delays which represent the pulse sequence (lines 1 and 2)

- The acquisition (lines 3-8) 


$\begin{array}{lllllllllll}0000 & \text { E000 } & 0080 & 0000 & 0000 & 0000 & 0000 & 0080 & 0000 & \text { D0 } & \\ 0000 & \text { E000 } & 0000 & 0000 & 0000 & 0001 & 0100 & 0080 & 0001 & \text { P1 } & 1 \\ 0000 & \text { E000 } & 0000 & 0000 & 0000 & 0000 & 0000 & 0020 & 0002 & \text { D2 } & \\ 0000 & \text { E000 } & 2000 & \text { FFFF } & \text { FFFA } & 0000 & 0000 & 0020 & 0003 & \text { A } & 3 \\ 0000 & \text { E000 } & 0000 & 0000 & 0000 & 0000 & 0000 & 0020 & 0004 & \text { AL } & 3 \\ 0000 & \text { E000 } & 2040 & \text { FFFF } & \text { FFFE } & 0000 & 0000 & 0020 & 0005 & \text { A } & 3 \\ 0000 & \text { E000 } & 0000 & \text { FFFF } & \text { FFFE } & 0000 & 0000 & 0020 & 0006 & \text { A } & 3 \\ 0000 & \text { E000 } & 0020 & \text { FFFF } & \text { FFFE } & 0000 & 0000 & 0020 & 0007 & \text { A } & 3 \\ 0002 & 3005 & 0000 & 0000 & 0000 & 0000 & 0000 & 0020 & 0008 & \text { AL } & 3 \\ 0000 & \text { E800 } & 0000 & 0000 & 0000 & 0000 & 0000 & 0000 & 0009 & \text { D3 } & \\ 0000 & 200 A & 0000 & 0000 & 0000 & 0000 & 0000 & 0000 & 000 A & \text { D5 } & \end{array}$

Figure 5.3: PULSE_ACQ.MIC, a pulse program for a simple one-dimensional proton experiment.

- Interrupt to the VAXStation II and extra line of microcode (lines 9-10)

Each line is required to have a microsequencer instruction in bits 111-108. The first line, the recycle delay D0, usually has bit 87 set to reset the SAkit data memory address back to zero. This is necessary any time more than one average is done. Otherwise, the data from each average will be written sequentially into the SAkit data memory rather than being co-added. Bit 7 for ENI deblanking is also set. This is necessary because there is a proton pulse in the next line and the ENI deblanking gate has a settling time $\gg 1$ microsecond. The only other bits that need to be set on this line are bits 79-48 which contain the timing bits for this line. This doesn't need to be set in the pulse program microcode because it is set by the TECMAG program.

The second line, labeled $\mathrm{P} 1$, is a proton pulse. This line must have 3 bits set bits 24 and 22 for the two RF gates and bit 7 for ENI deblanking. The timing bits are set in the TECMAG program and the phase of the proton pulse is set based on the values in phase table 1 . If no phase table is specified for this pulse, then one 
of the bits 23-20 must be set in the pulse program microcode to specify the phase or the pulse. Line 2, D2, is the ringdown delay before starting data acquisition. At this point, the receiver deblanking is turned on (bit 5) and the ENI deblanking is turned off (bit 7).

The next six lines comprise the data acquisition sequence shown in Figure 5.4. The lines with mnemonic " $A$ " must have the timing word set as shown, while the lines with mnemonic "AL" are set by the software, based on the dwell time. The six lines for acquisition in the pulse program (lines 3-8) all have the RB bit (bit 5) on. Also, they all use phase table \#3, as specified in column 6 of the descriptor at the end of the line. Line 3 digitizes the first point with the A to D bit (bit 93) set for 0.6 microseconds. The next line is a delay set by the TECMAG program which, when added to 0.6 microseconds, equals the dwell time. The next four lines digitize the remaining points. Line 5 triggers the $A$ to $D$ converter again (bit 93) and the CONV bit (bit 86) to add the previously digitized word to the value in the SAkit memory. Following this is a 0.2 microsecond delay in line 6 , then in line 7 the EOC bit (bit 85) is triggered to complete the acquisition of a point by writing the result of the addition triggered by the CONV bit to the SAkit data memory and incrementing the SAkit data memory address. Line 8 is a delay which when added to 0.6 microseconds equals the dwell time. Line 8 also has a conditional loop instruction in bits 111-108. It uses loop counter 2 (designated by bit 113) to determine whether or not to loop back to line 5 (designated in bits 106-96) and acquire another point. The TECMAG software assumes that loop counter 2 will be used to determine the number of points to acquire per FID. This loop counter is loaded by the TECMAG program with the two's complement value of the number 


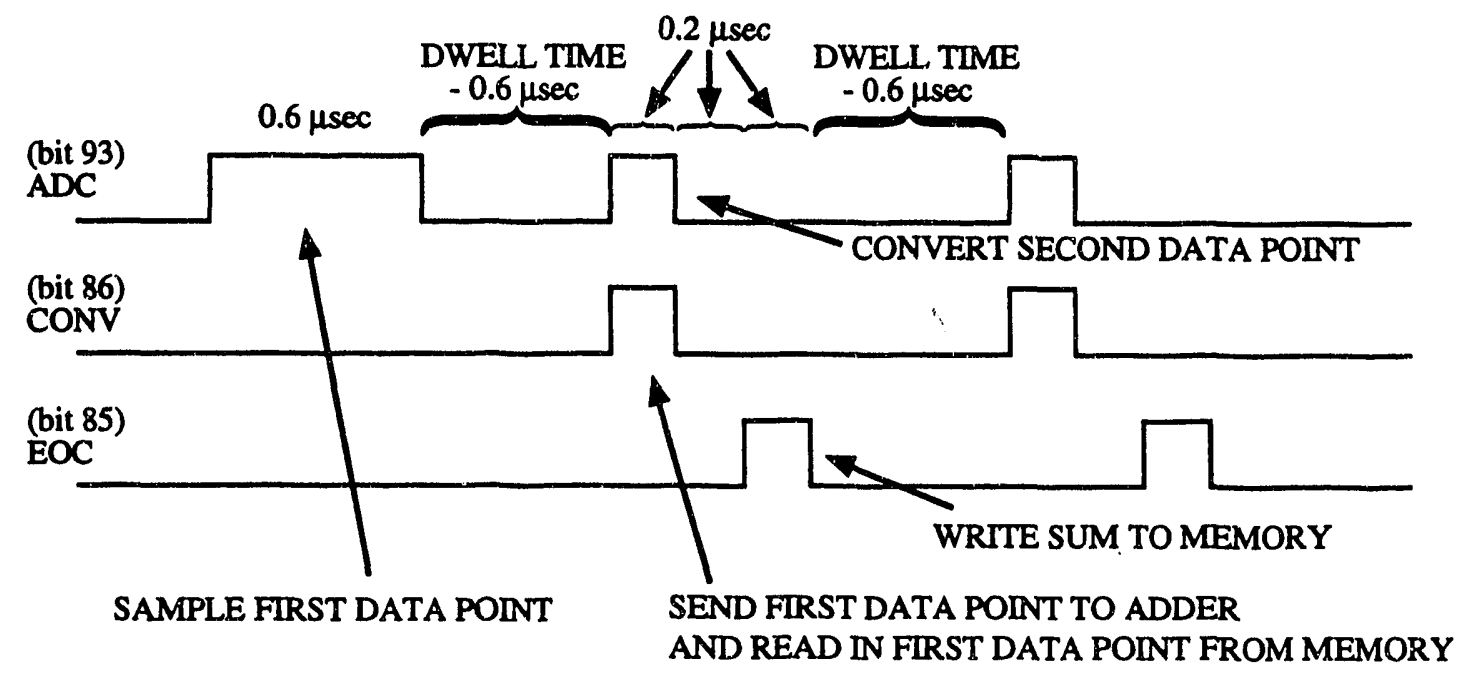

Figure 5.4: Timing diagram for data acquisition with the SAkit. All pulses are generated by the PULSkit.

of points to be acquired (NB). When line 8 is executed, if loop counter 2 has a nonzero value, then the value in loop counter 2 is incremented and line 5 is executed. Otherwise, if loop counter 2 has the value 0 , then line 9 is executed. The other bits that need to be set in these six data acquisition lines are the receiver phase bits. The receiver phase is specified in phase table 3 . If no phase table is specified here, a particular phase must be hard-coded into the microcode. For an $\mathrm{X}$ receiver phase, bit 92 must be set to "0" and bits 91 and 90 must be "1", for $Y$ - bits 92,91 , and 90 must be set to "0", for $\overline{\mathrm{X}}$ - bits 92 and 90 must be " 1 " and bit 91 must be "0", and for $\bar{Y}$ - bits 92 and 91 must be set to " 1 " and bit 90 must be " 0 " in lines 3-8 of the microcode. Generally, the data acquisition sequence for any pulse sequence can be specified by using the six lines 3-8 in this pulse program, and simply numbering them according to their location in a pulse sequence and changing the loop branch address (bits 106-96) in the last of the six lines. 
Line 9, D3, sends an interrupt to the VAXStation II by setting bit 107, the ATTN bit. The VAXStation II then calls the interrupt service routine in the TECMAG program, which immediately stops the PULSkit, and, if necessary uploads data from the SAkit memory to the VAXStation II, updates the pulse and receiver phases, loads the modified pulse program, and restarts it for the next scan. The last line in the pulse program is just a safety measure, so that if the VAXStation II fails to respond to the ATTN bit interrupt in line 9, the PULSkit goes into an infinite loop with the transmitter gated off.

\subsubsection{Example of Use of the TECMAG Program}

The TECMAG program is located in the directory DUBO:[TECMAG] and can be started by setting this to the default directory and typing "RUN TECMAG" at the VMS prompt. When the program is started, a small window is created which is used to display a running count of the number of averages completed (NA) and the number of FIDs completed (NF) so far in the experiment. Several subdirectories exist for files related to the TECMAG program. [TECMAG.MIC] contains files containing microcode pulse programs. These files must end with the suffix ".MIC". The subdirectory [TECMAG.DATA] contains the data files written by the TECMAG program. [TECMAG.PHASE] contains the phase tables used by the TECMAG program when specified in a pulse program. The format of the phase tables for a pulse or the receiver is simply a file named " $\chi$.PHASE" (where $\chi$ is a number or letter corresponding to that specified in the descriptor on a line of a pulse program) which contains lines which each have a single number on them : 0 for $\mathrm{X}$ phase, 1 for $\mathrm{Y}, 2$ for $\overline{\mathrm{X}}$, and 3 for $\overline{\mathrm{Y}}$. These tables are read by the TECMAG 
program when a pulse program is read in using the "PG" command. If you wish to change the phase table for a pulse program after it is read into the TECMAG program, you must edit the file "[TECMAG.PHASE] $\chi$.PHASE" for the particular phase tables you wish to change and do the "PG" command again to read the new phase tables. No other parameter values are changed by executing the "PG" command.

An example of using the TECMAG program to run an experiment and collect data using the PULSE_ACQ pulse program is shown below. User input is indicated by bold-faced type. All time durations in the program are entered and written out in microseconds.

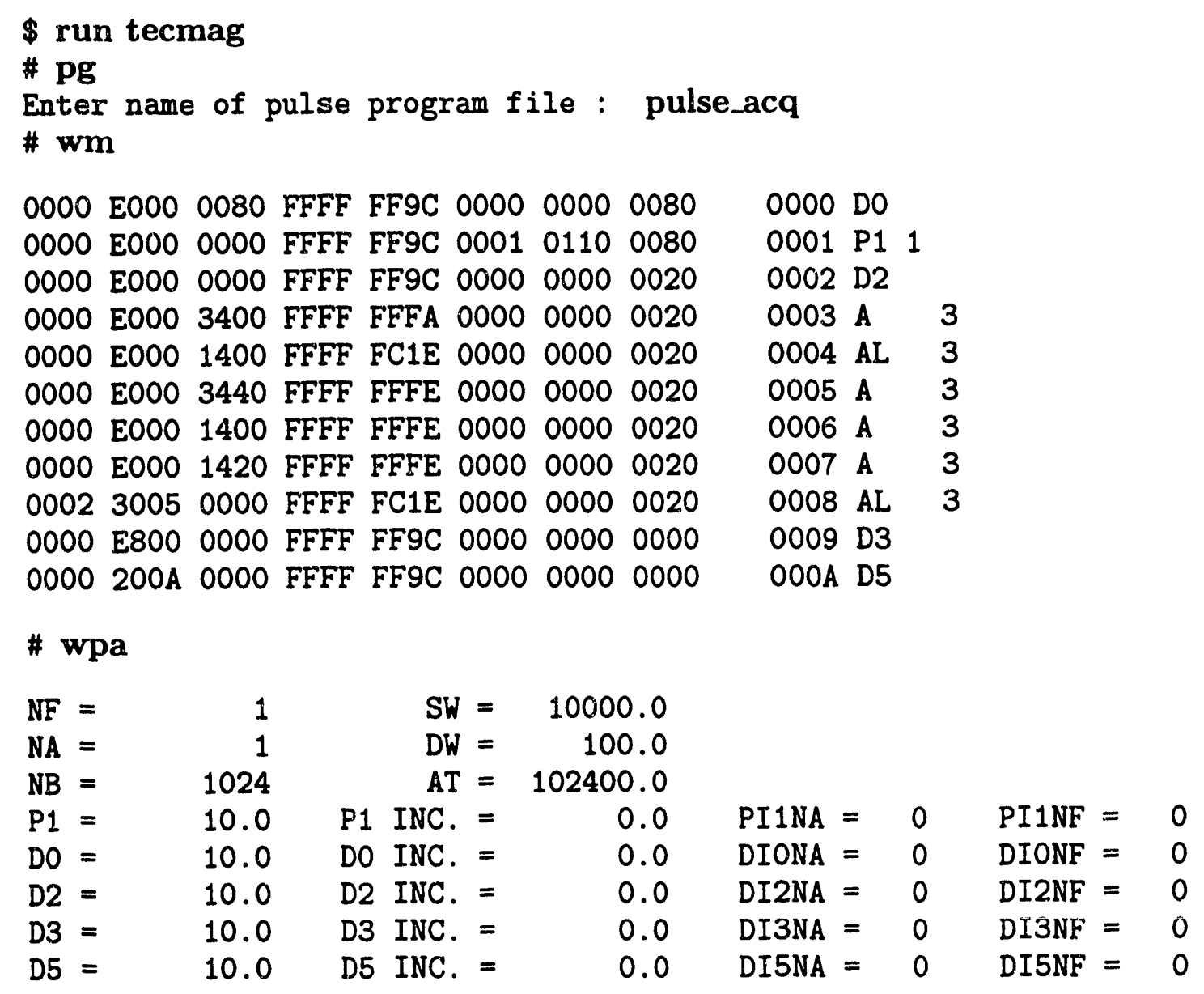


\# wph

FSNA $=0$ FSNF $=0$ PSRNA $=0$ PSRNF $=0$ PSINA $=0$

PH1 : $0 \begin{array}{llll}0 & 1 & 2 & 3\end{array}$

PH3 : $0 \begin{array}{llll}0 & 1 & 2 & 3\end{array}$

There are several things to note at this point. When the TFCMAG program is started, all pulses and delays are initialized to 10 microseconds, and the other acquisition parameters are initialized to the values shown above by the "wpa" command. The files "[TECMAG.PHASE]1.PHASE" and "[TECMAG.PHASE]3.PHASE" have the values $0,1,2$, and 3 in them and are read in when the "pg" command is executed. Also note that the pulse program as printed out by the "wm" command has been updated to contain the correct timing words and to have the correct pulse and receiver phases, based on the first values in the phase tables. The next thing to do before attempting to run an experiment is to set the pulse and delay lengths, the sweep width, the number of points, and the number of averages to the desired values.

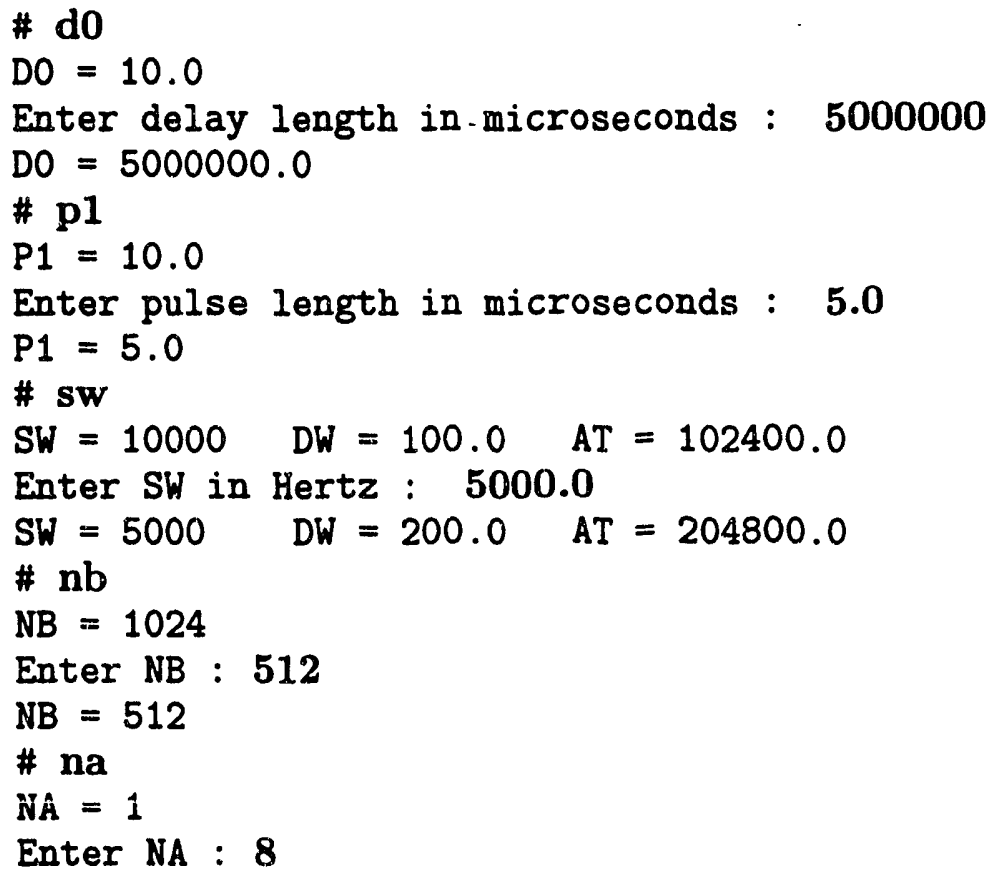


$\mathrm{NA}=8$

\# wm

$\begin{array}{lllllllllll}0000 & \text { E000 } & 0080 & \text { FD05 } & \text { OF80 } & 0000 & 0000 & 0080 & 0000 & \text { D0 } & \\ 0000 & \text { E000 } & 0000 & \text { FFFF FFCE } & 0001 & 0110 & 0080 & 0001 & \text { P1 } & 1 & \\ 0000 & \text { E000 } & 0000 & \text { FFFF FF9 } & 0000 & 0000 & 0020 & 0002 & \text { D2 } & \\ 0000 & \text { E000 } & 3400 & \text { FFFF FFFA } & 0000 & 0000 & 0020 & 0003 & \text { A } & 3 \\ 0000 & \text { E000 } & 1400 & \text { FFFF F836 } & 0000 & 0000 & 0020 & 0004 & \text { AL } & 3 \\ 0000 & \text { E000 } & 3440 & \text { FFFF FFFE } & 0000 & 0000 & 0020 & 0005 & \text { A } & 3 \\ 0000 & \text { E000 } & 1400 & \text { FFFF FFFE } & 0000 & 0000 & 0020 & 0006 & \text { A } & 3 \\ 0000 & \text { E000 } & 1420 & \text { FFFF FFFE } & 0000 & 0000 & 0020 & 0007 & \text { A } & 3 \\ 0002 & 3005 & 0000 & \text { FFFF F836 } & 0000 & 0000 & 0020 & 0008 & \text { AL } & 3 \\ 0000 & \text { E800 } & 0000 & \text { FFFF FF9C } & 0000 & 0000 & 0000 & 0009 & \text { D3 } & \\ 0000 & 200 A & 0000 & \text { FFFF FF9C } & 0000 & 0000 & 0000 & 000 A & \text { D5 } & \end{array}$

\# wpa

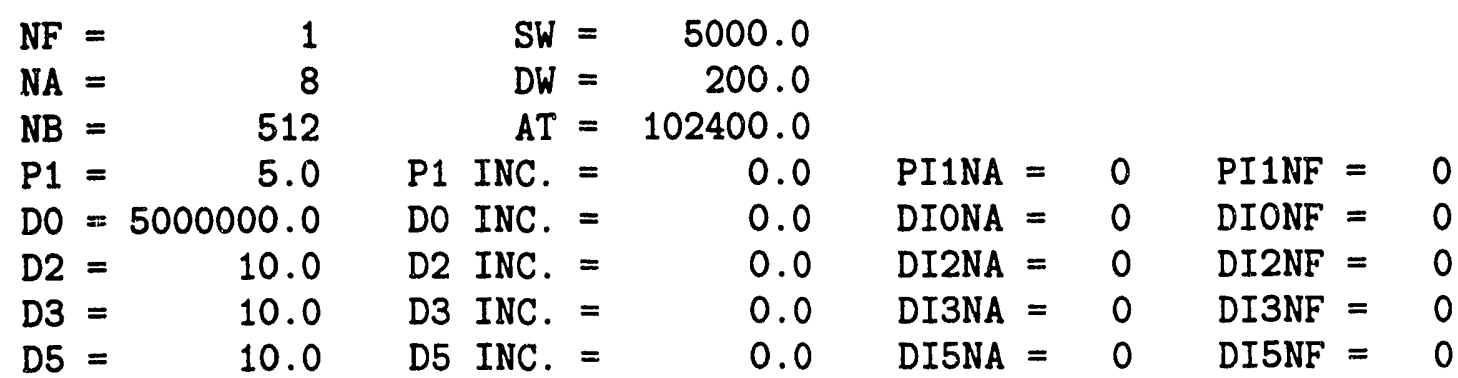

\# wph

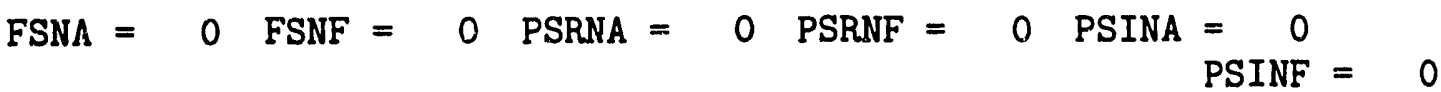

PH1 : $\begin{array}{llll}0 & 1 & 2 & 3\end{array}$

PH3 : $0 \begin{array}{llll}0 & 1 & 2 & 3\end{array}$

\# zg

Enter name of data file: 052592

\#

The "zg" command above zeros the SAkit data memory, loads the pulse program exactly as written above by the "wm" command into the PULSkit memory and starts execution of the pulse program. Four files are created in the directory "[TECMAG.DATA]". The first, "052592.par", contains acquisition parameters in a format that can be read by the data processing program "NMR1D" on the VAXStation II or "nmrX" on the Stardent computer (see Appendix A). The second, 
"052592.acq", contains the output from the TECMAG program commands "wm", "wlc", "wpa", and "wph" and can be read at any time back into the TECMAG program by the "rp" command to set the pulse program and all parameters exactly as they were at the beginning of this experiment. The third, "052592end.acq", contains the same information as the file "052592.acq" except the parameters have the values they took on the last scan prior to completion of the experiment. This file is useful for verifying that pulse or delay increments or phase tables were done correctly. The last file, "052592.data" contains the data from the experiment. It is written a a binary file of 32-bit integers with record length 512 bytes. The real buffer is written first, followed by the imaginary buffer. Phase tables are considered to be cyclical. For example, if there are 8 averages and only four entries in the phase table, then on the fifth scan the first entry in the phase table will be reused.

\subsubsection{More Pulse Programs}

Figure 5.5 shows a pulse program for a simple two-pulse COSY experiment. It is identical to the one-dimensional experiment in Figure 5.3 except for the addition of lines 2 and 3 with a delay, D1, and a second pulse, P1. The same steps as just described for the one-dimensional experiment need to be done to read the pulse program into the TECMAG program and set the parameters. The additional steps that need to be done for a two-dimensional experiment are to set D1 to an initial value, to set an increment for $\mathrm{D} 1$ (the dwell time in $\mathrm{t}_{1}$ ), and to set the number of $t_{1}$ points to acquire. Typical values for these parameters would be set as follows :

\section{\# d1}

$D 1=10.0$

Enter delay length in microseconds : 1.0 


$\begin{array}{lllllllllll}0000 & \text { E000 } & 0080 & 0000 & 0000 & 0000 & 0000 & 0080 & 0000 & \text { D0 } & \\ 0000 & \text { E000 } & 0000 & 0000 & 0000 & 0001 & 0100 & 0080 & 0001 & \text { P1 } & 1 \\ 0000 & \text { E000 } & 0000 & 0000 & 0000 & 0000 & 0000 & 0080 & 0002 & \text { D1 } & \\ 0000 & \text { E000 } & 0000 & 0000 & 0000 & 0001 & 0100 & 0080 & 0003 & \text { P1 } & 1 \\ 0000 & \text { E000 } & 0000 & 0000 & 0000 & 0000 & 0000 & 0020 & 0004 & \text { D2 } & \\ 0000 & \text { E000 } & 2000 & \text { FFFF } & \text { FFFA } & 0000 & 0000 & 0020 & 0005 & \text { A } & 3 \\ 0000 & \text { E000 } & 0000 & 0000 & 0000 & 0000 & 0000 & 0020 & 0006 & \text { AL } & 3 \\ 0000 & \text { E000 } & 2040 & \text { FFFF } & \text { FFFE } & 0000 & 0000 & 0020 & 0007 & \text { A } & 3 \\ 0000 & \text { E000 } & 0000 & \text { FFFF } & \text { FFFE } & 0000 & 0000 & 0020 & 0008 & \text { A } & 3 \\ 0000 & \text { E000 } & 0020 & \text { FFFF } & \text { FFFE } & 0000 & 0000 & 0020 & 0009 & \text { A } & 3 \\ 0002 & 3007 & 0000 & \text { D000 } & 0000 & 0000 & 0000 & 0020 & 000 A & \text { AL } & 3 \\ 0000 & \text { E800 } & 0000 & 0000 & 0000 & 0000 & 0000 & 0000 & 000 B & \text { D3 } & \\ 0000 & 200 A & 0000 & 0000 & 0000 & 0000 & 0000 & 0000 & 000 C & \text { D5 } & \end{array}$

Figure 5.5: A pulse program for a simple two-dimensional proton COSY experiment.

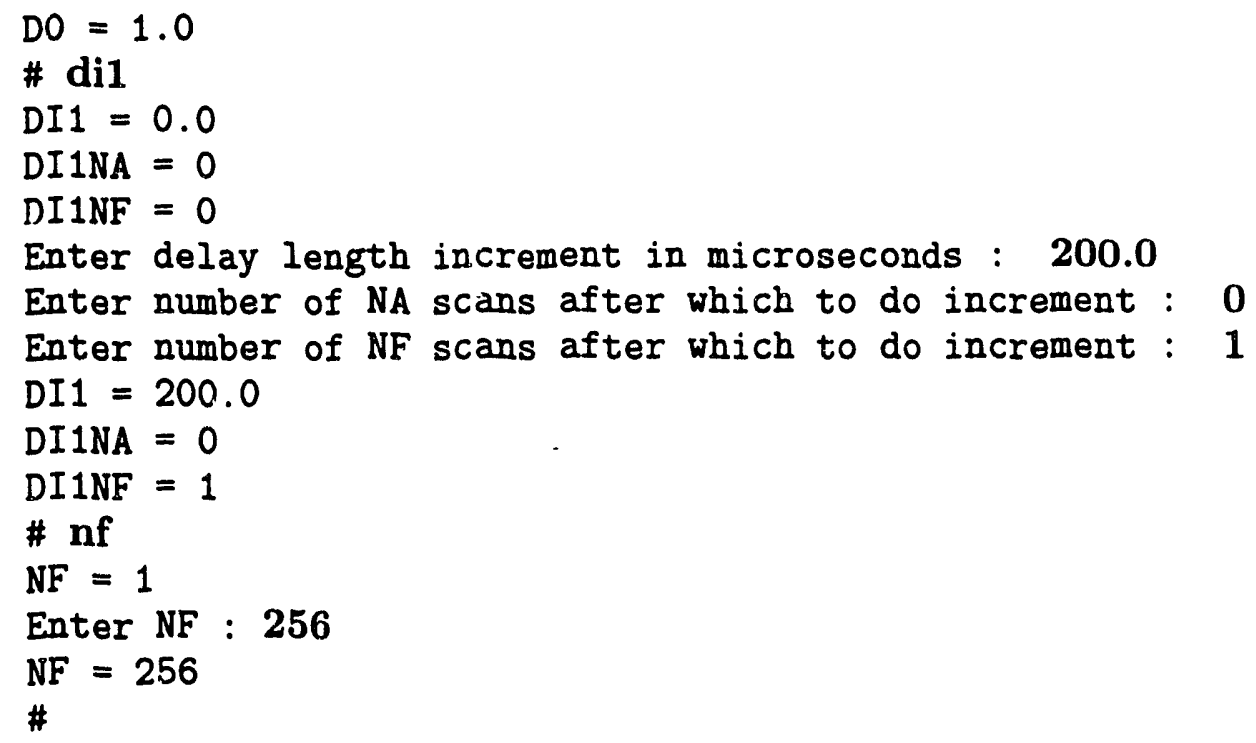

The above commands would set up the two dimensional experiment to have a sweep width of $5000 \mathrm{~Hz}$ in $t_{1}$ and to collect 256 points in $t_{1}$, that is to increment D1 by 200.0 microseconds after each fid is collected (DI1NF = 1). After each FID is collected (after each NF), the FID is uploaded to the VAXStation II and written sequentially to a file on disk in the same format as in a one-dimensional experiment. 


$\begin{array}{lllllllllll}0000 & E 000 & 0080 & 0000 & 0000 & 0000 & 0000 & 0080 & 0000 & \text { D0 } & \\ 0000 & \text { E000 } & 0000 & 0000 & 0000 & 0001 & 0110 & 0080 & 0001 & \text { P1 } & \\ 0004 & 3001 & 0000 & 0000 & 0000 & 0000 & 0000 & 0080 & 0002 & \text { D2 } & 3 \\ 0000 & \text { E800 } & 0000 & 0000 & 0000 & 0000 & 0000 & 0000 & 0003 & \text { D3 } & \\ 0000 & 2004 & 0000 & 0000 & 0000 & 0000 & 0000 & 0000 & 0004 & \text { D5 } & \end{array}$

Figure 5.6: A pulse program for producing a rapid train of pulses for observation on an oscilloscope.

As another example of a pulse program, the pulse program in Figure 5.6 can be used to look at pulses on an oscilloscope. It has no acquisition sequence, but uses a loop counter to provide a train of pulses spaced by a delay. Typically the delay D0 would be set $>0.1$ second, $\mathrm{P} 1$ would be set to 10 microseconds and D2 would be set to $>100$ microseconds. Then loop counter 3 (specified by setting bit 114 on line 2 of the microcode) would be set to a large value using the TECMAG command "lc3" and a train of pulses separated by a delay would be produced. The loop counters are only 16 bits so that the maximum value for "lc3" is 65535 . It is not possible to use "NA" to produce a train of pulses for the scope, since NA is incremented each time the ATTN bit (bit 107) is set and the VAXStation II calls the interrupt service routine, and to do this takes $\sim 0.1$ seconds.

The next example is a two-dimensional, even-quantum excitation experiment shown in Figure 5.7. The experiment was used to obtain 6-QT vs. 1-QT correlation spectra of $1,1,1,6,6,6-\mathrm{d}_{6} n$-hexane dissolved in a liquid crystal with $\mathrm{CW}$ deuterium decoupling, as described in references $[36,37,38]$. The pulse sequence for this experiment is :

$$
\left(\frac{\pi}{2}\right)_{\phi}-\frac{\tau_{1}}{2}-(\pi)_{\phi}-\frac{\tau_{1}}{2}-\left(\frac{\pi}{2}\right)_{\phi}-\frac{t_{1}}{2}-(\pi)_{\chi}-\frac{t_{1}}{2}-\left(\frac{\pi}{2}\right)_{x}-\frac{\tau_{2}}{2}-(\pi)_{x}-\frac{\tau_{2}}{2}-t_{2} .
$$

TPPI was use to excite and separate up to 8 quantum transitions by incrementing 
the phase of pulses, $\phi$, by $22.5^{\circ}$ in step with incrementation of $t_{1}$. The phase $\chi$ was cycled through $\mathrm{X}, \mathrm{Y}, \overline{\mathrm{X}}$, and $\overline{\mathrm{Y}}$ and the receiver phase was not cycled. The results of the TECMAG commands "wm", "wpa", and "wph" just prior to starting the experiment are shown below :

\# wm

0000 E000 0080 FFFF FF38 000000000000 0000 E000 0000 FC20 2DC0 000000000080 0000 E000 0000 FFFF FF93 000101100091 0000 E000 0000 FFFF 1B5E 000000000091 0000 E000 0000 FFFF FF26 000101100091 0000 E000 0000 FFFF 1B5E 000000000091 0000 E000 0000 FFFF FF93 000101100091 0000 E000 0000 FFFF FFD8 000040000091 0000 E000 0000 FFFF FF26 000101100091 0000 E000 0000 FFFF FFD8 000000000091 0000 E000 0000 FFFF FF93 000101100091 0000 E000 0000 FFFF C568 000000000091 0000 E000 0000 FFFF FF26 000101100091 0000 E000 0000 FFFF C568 000040000091 0000 E000 3400 FFFF FFFA 000000000031 0000 E000 1400 FFFF FFOC 000000000031 0000 E000 3440 FFFF FFFE 000000000031 0000 E000 1400 FFFF FFFE 000000000031 0000 E000 1420 FFFF FFFE 000000000031 000230100000 FFFF FFOC 000000000031 0000 E800 0000 FFFF FF9C 000000000000 0000 200A 0000 FFFE 7960000000000000

$\begin{array}{llll}0000 & \text { DA } & & \text { I } \\ 0001 & \text { DO } & & \text { R } \\ 0002 & \text { P1 } & 1 & \\ 0003 & \text { D4 } & & \\ 0004 & \text { P2 } & 1 & \\ 0005 & \text { D4 } & & \\ 0006 & \text { P1 } & 1 & \\ 0007 & \text { D1 } & \\ 0008 & \text { P2 } & 2 \\ \text { 0009 } & \text { D1 } & \\ \text { 000A } & \text { P1 } & 1 \\ \text { 000B } & \text { D6 } & \\ \text { 000C } & \text { P2 } & 1 & \\ 000 D & \text { D6 } & \\ \text { 000E } & \text { A } & 3 \\ \text { 000F } & \text { AL } & 3 \\ \text { 0010 } & \text { A } & 3 \\ \text { 0011 } & \text { A } & 3 \\ \text { 0012 } & \text { A } & 3 \\ \text { 0013 } & \text { AL } & 3 \\ \text { 0014 } & \text { D3 } & \\ \text { 0015 } & \text { D5 } & \end{array}$

\section{\# wpa}

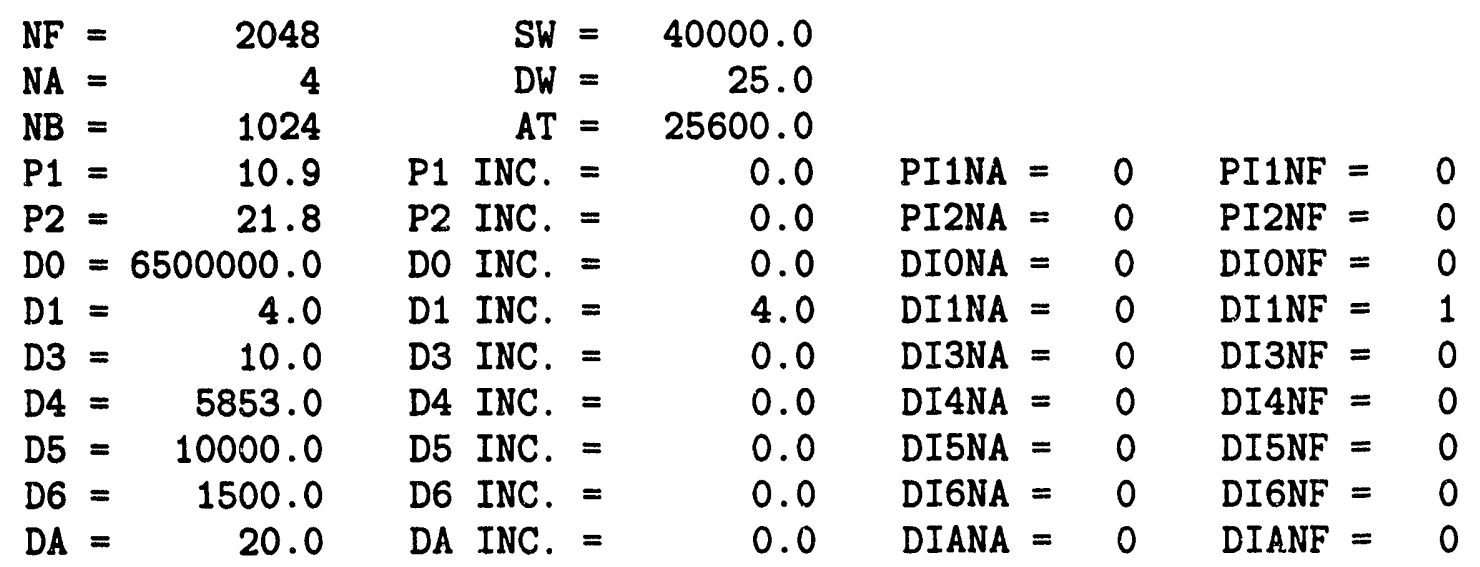


FSNA $=0$ FSNF $=0$ PSRNA $=0$ PSRNF $=16 \quad$ PSINA $=\begin{aligned} & 0 \\ & \text { PSINF }=1\end{aligned}$

\# wph

FSNA $=0$ FSNF $=0$ PSRNA $=0$ PSRNF $=16$ PSINA $=0$

PH1 : 0

PH3 : $0 \begin{array}{llll}0 & 1 & 2 & 3\end{array}$

PH3 : 0

The things to note here are the use of the phase shift reset, increment and toggle bits. The phase shift reset bit is set every 16 FID's (after it should be back to zero anyway) and the phase shift increment bit is set at the start of every FID to implement the TPPI scheme. The phase shift toggle bit is set in lines 7 and D to toggle the digital phase shifter out of line and then back in line, so that the pulses between those lines will have the quadrature phases specified by the phase tables, and not the phase specified by the digital phase shifter.

The final pulse program example is a Carr-Purcell-Meiboom-Gill echo train acquisition $[86,87]$ to demonstrate how single point acquisition is done. The pulse program for this experiment is shown in Figure 5.8. Acquisition of a single point is done at the tops of the echos between successive $\pi$ pulses. The dwell time for such an experiment should be set to be the same as D2. NB will still control the number of points that are acquired using loop counter 2 to loop, do another $\pi$ pulse and delay, and acquire another point.

\subsubsection{Miscellany about the TECMAG Program}

The upload command "UL" allows the user to upload the data at any point in the experiment. If an experiment is running when the "UL" command is typed, the next time a scan is finished (the next time the ATTN bit is set in the pulse program), the 


\begin{tabular}{|c|c|c|c|c|c|c|c|c|c|c|}
\hline 0000 & $\mathrm{E} 000$ & 0080 & 0000 & 0000 & 0000 & 0000 & 0080 & 0000 & $\mathrm{DA}$ & \\
\hline 0000 & E000 & 000 & 000 & 0000 & 0000 & 0000 & 0080 & 0001 & DO & \\
\hline 0000 & EOOO & 0000 & 0000 & 0000 & 0001 & 0100 & 0091 & 0002 & P1 & 1 \\
\hline 0000 & E000 & 0000 & 0000 & 0000 & 0000 & 0000 & 0091 & 0003 & D4 & \\
\hline 0000 & E000 & 0000 & 0000 & 0000 & 0001 & 0100 & 0091 & 0004 & P2 & 1 \\
\hline 0000 & $\mathrm{E} 000$ & 0000 & 0000 & 0000 & 0000 & 0000 & 0091 & 0005 & $\mathrm{D} 4$ & \\
\hline 0000 & E000 & 0000 & 0000 & 0000 & 0001 & 0100 & 0091 & 0006 & P1 & 1 \\
\hline 0000 & E000 & 0000 & 0000 & 0000 & 0000 & 4000 & 0091 & 0007 & D1 & \\
\hline 0000 & EOOO & 0000 & 0000 & 0000 & 0001 & 0100 & 0091 & 0008 & P2 & 2 \\
\hline 0000 & E000 & 0000 & 0000 & 0000 & 0000 & 0000 & 0091 & 0009 & D1 & \\
\hline 0000 & E000 & 0000 & 0000 & 0000 & 0001 & 0100 & 0091 & $000 \mathrm{~A}$ & P1 & 1 \\
\hline 0000 & E000 & 0000 & 0000 & 0000 & 0000 & 0000 & 0091 & $000 B$ & D6 & \\
\hline 0000 & $\mathrm{E} 000$ & 0000 & 0000 & 0000 & 0001 & 0100 & 0091 & $000 \mathrm{C}$ & $\mathrm{P} 2$ & 1 \\
\hline 0000 & EOOO & 0000 & 0000 & 0000 & 0000 & 4000 & 0020 & OOOD & D6 & \\
\hline 0000 & E000 & 2000 & FFFF & FFFA & 0000 & 0000 & 0020 & $000 \mathrm{E}$ & A & 3 \\
\hline 0000 & $\mathrm{E} 000$ & 0000 & 0000 & 0000 & 0000 & 0000 & 0020 & OOOF & $\mathrm{AL}$ & 3 \\
\hline 0000 & E000 & 2040 & FFFF & FFFE & 0000 & 0000 & 0020 & 0010 & A & 3 \\
\hline 0000 & $\mathrm{E} 000$ & 0000 & FFFF & FFFE & 0000 & 0000 & 0020 & 0011 & A & 3 \\
\hline 0000 & E000 & 0020 & FFFF & FFFE & 0000 & 0000 & 0020 & 0012 & A & 3 \\
\hline 0002 & 3010 & 0000 & 0000 & 0000 & 0000 & 0000 & 0020 & 0013 & $A L$ & 3 \\
\hline 0000 & $\mathrm{E} 800$ & 0000 & 0000 & 0000 & 0000 & 0000 & 0000 & 0014 & D3 & \\
\hline 0000 & 2015 & 0000 & 0000 & 0000 & 0000 & 0000 & 0000 & 0015 & D5 & \\
\hline
\end{tabular}

Figure 5.7: A pulse program for a TPPI multiple-quantum experiment. 


$\begin{array}{llllllllllll}0000 & \text { E000 } & 0080 & 0000 & 0000 & 0000 & 0000 & 0080 & 0000 & \text { D0 } & \\ 0000 & E 000 & 0000 & 0000 & 0000 & 0001 & 0100 & 0080 & 0001 & \text { P1 } & 1 \\ 0000 & E 000 & 0000 & 0000 & 0000 & 0000 & 0000 & 00 A 0 & 0002 & \text { D2 } & \\ 0000 & \text { E000 } & 0000 & 0000 & 0000 & 0001 & 0100 & 0080 & 0003 & \text { P2 } & 2 \\ 0000 & \text { E000 } & 0000 & 0000 & 0000 & 0000 & 0000 & 0020 & 0004 & \text { D2 } & \\ 0000 & \text { E000 } & 2000 & \text { FFFF } & \text { FFFA } & 0000 & 0000 & 0020 & 0005 & \text { A } & 3 \\ 0000 & \text { E000 } & 0000 & 0000 & 0000 & 0000 & 0000 & 0020 & 0006 & \text { AL } & 3 \\ 0000 & \text { E000 } & 0000 & 0000 & 0000 & 0001 & 0100 & 0080 & 0007 & \text { P2 } & 2 & \\ 0000 & \text { E000 } & 0000 & \text { D000 } & 0000 & 0000 & 0000 & 0020 & 0008 & \text { D2 } & \\ 0000 & \text { E000 } & 2040 & \text { FFFF } & \text { FFFE } & 0000 & 0000 & 0020 & 0009 & \text { A } & 3 \\ 0000 & \text { E000 } & 0000 & \text { FFFF } & \text { FFFE } & 0000 & 0000 & 0020 & 000 A & \text { A } & 3 \\ 0000 & \text { E000 } & 0020 & \text { FFFF } & \text { FFFE } & 0000 & 0000 & 0020 & 000 B & \text { A } & 3 \\ 0002 & 3007 & 0000 & 0000 & 0000 & 0000 & 0000 & 0020 & 000 C & \text { AL } & 3 \\ 0000 & \text { E800 } & 0000 & 0000 & 0000 & 0000 & 0000 & 0000 & 000 D & \text { D3 } & \\ 0000 & 200 F & 0000 & 0000 & 0000 & 0000 & 0000 & 0000 & 000 E & \text { D5 } & \end{array}$

Figure 5.8: A pulse program for single point acquisition during a Carr-PurcellMeiboom-Gill echo train.

data in the SAkit memory is uploaded aud written out to the file "TEMP.DATA". A "TEMP.PAR" file is also created for use with the data processing programs. If no experiment is running when the "UL" command is typed, then "NB" points are uploaded from the SAkit memory and user is prompted for a filename to which the data should be written.

The user account on the VAXStation II needs extra privileges besides the default ones to run the TECMAG program. Some of these are specified in the Tecmag, Inc. manual for Pulse123 software [88]. If the users of the TECMAG program can be trusted, then it is usually easier to just give the account(s) which use the TECMAG program "SYSPRV" privilege.

Two common problems can occur, which result in strange error messages from the TECMAG program. The first occurs if the Tecmag unit does not have fower to it. When the "RUN TECMAG" command is issued, an error message "Reset 
DR11-W failed" will appear. Second, if the $10 \mathrm{MHz}$ clock for the Tecmag unit is either of insufficient amplitude or is not connected, the TECMAG program will appear to run normally until a "GO" or "ZG" command is executed, at which time the program will crash with the error message "LDLC failed".

Some cautions for use of the TECMAG program :

- The recycle delay (usually D0) should not be set less than 0.1 seconds. If this is absolutely necessary, it should be tried first with the RF amplifier(s) off, so that problems with the VAXStation II or Tecmag PULSkit don't result in long pulses which could damage a probe or sample. Alternatively, it is possible to use a loop counter to do a fast recycle delay without having to set the ATTN bit and send an interrupt to the VAXStation II. If this is the case, all phase cycling must be hard-coded into the pulse program, since software phase updates using phase tables can only be done in the TECMAG program when the interrupt service routine is called by setting the ATTN bit in the pulse program.

- Pulse lengths should not be set longer than about 100 microseconds, unless you know what you are doing, since such pulses could damage a probe or sample.

- Always check the pulse program and parameters using the "wrn", "wpa", and "wph" commands before running a pulse program. It ray also be prudent to run the pulse program with the RF amplifiers turned off to test that it does what it should before running an experiment.

- It may be useful to type the "wm" command during recycle delays between 
scans to see that the phases of pulses and the receiver are being updated properly. 


\section{Appendix A}

\section{Software}

\section{A.1 Data Processing}

In addition to the TECMAG program described in the previous chapter, several programs were written or modified to process and display data on different computers.

On the VAXStation II and MicroVAX II computers :

- NMR1D - A program originally written by Dieter Suter to process and display one-dimensional NMR data. It has been modified to enhance its capabilities. It can read the ".par" and ".data" files written by the TECMAG program.

- NMR2D - A program originally written by Dieter Suter to process and display two-dimensional NMR data. Very slow for processing large data sets.

- NMR2DPLOT - A program derived from the NMR2D program which reads in processed two-dimensional NMR data and plots it to the screen or to an 
HP7550 plotter. Better plotting and interactive peak-picking than NMR2D.

- 2DFT - A program originally written by Steve Rucker. It reads and processes two-dimensional NMR data files written by the TECMAG program. The output file from this program can be read by the NMR2DPLOT program.

On the Stardent computer :

- nmrX - A program for processing and display of one-dimensional NMR data. It can read the ".par" and ".data" files produced by the TECMAG program, as well as Bruker data and binary data stored as float's with alternating real and imaginary points. Outputs include PostScript and Adobe Illustrator formats.

- nmrX_2D - A program for processing and display of two-dimensional NMR data. It can read the ".par" and ".data" files produced by the TECMAG program, as well as Bruker data and binary data stored as float's with alternating real and imaginary points. Outputs include PostScript, Adobe Illustrator, and AVS field formats.

\section{A.2 Simulation}

The programs used for the simulations in Chapters 3 and 4 can be found on the Stardent computer. The programs for the mean-field simulations in Chapter 3 are located in the directory "/opt/pines/rosen/marksalks/rmsdev/sources" and are in subdirectories named for the model and the number of adjustable parameters used in the simulation. The model names used are "burnell" for Model A, "samulski" for Model B, "inertia" for Model C, and "photinos" for Model D. The program for 
the simulations in Chapter 4 is in the directory "/opt/pines/rosen/thf/sim" and is named "thf". 


\section{Bibliography}

[1] D. J. Photinos, E. T. Samulski, and H. Toriumi, J. Phys. Chem. 94, 4688 (1990).

[2] A. Abragam, Principles of Nuclear Magnetism (Oxford University Press, Oxford, 1962).

[3] C. P. Slichter, Principles of Magnetic Resonance, $3^{\text {rd }}$ edition (Springer Verlag, New York, 1.989).

[4] R. R. Ernst, G. Bodenhausen, and A. Wokaun, Principles of Nuclear Magnetic Resonance in One and Two Dimensions (Clarendon Press, Oxford, 1987).

[5] M. Munowitz, Coherence and NMR (John Wiley and Sons, New York, 1988).

[6] M. Goldman, Quantum Description of High-Resolution NMR in Liquids (Clarendon Press, Oxford, 1988).

[7] T. C. Farrar and E. D. Becker, Pulse and Fourier Transform NMR (Academic Press, New York, 1971).

[8] J. J. Sakurai, Modern Quantum Mechanics (Benjamin/Cummings Publishing Company, Inc., Menlo Park, CA, 1985). S. F. Tuan, ed.

[9] M. E. Rose, Elementary Theory of Angular Momentum (Wiley, New York, 1957).

[10] B. L. Silver, Irreducible Tensor Methods (Academic Press, New York, 1976).

[11] M. Mehring, Principles of High Resolution NMR in Solids (Springer Verlag, Berlin, 1983).

[12] U. Haeberlen, High Resolution NMR in Solids : Selective Averaging (Academic Press, New York, 1976). 
[13] J. W. Emsley and J. C. Lindon, NMR Spectroscopy using Liquid Crystal Solvents (Pergamon Press, Oxford, 1975).

[14] J. Jeener, in Amperè International Summer School (Basko Polje, Yugoslavia, 1971).

[15] W. P. Aue, E. Bartholdi, and R. R. Ernst, J. Chem. Phys. 64, 2229-2246 (1976).

[16] P. G. de Gennes, The Physics of Liquid Crystals (Clarendon Press, Oxford, 1974).

[17] ed. G. W. Gray, Thermotropic Liquid Crystals (John Wiley \& Sons, Chichester, 1987).

[18] G. Vertogen and W. H. de Jeu, Thermotropic Liquid Crystals, Fundamentals (Springer-Verlag, Berlin, 1988).

[19] ed. F. D. Saeva, Liquid Crystals - The Fourth State of Matter (Marcel Dekker, Inc., New York, 1979).

[20] eds. G. R. Luckhurst and G. W. Gray, The Molecular Physics of Liquid Crystals (Academic Press, London, 1979).

[21] eds. C. Hilsum and E. P. Raynes, Liquid Crystals : Their Physics, Chemistry and Applications (The Royal Society, London, 1983).

[22] S. Chandrasekhar, Liquid Crystals (Cambridge University Press, Cambridge, 1977).

[23] S. Chandrasekhar and G. S. Ranganath, Rep. Prog. Phys. 53, 57-84 (1990).

[24] T. Seideman, Rep. Prog. Phys. 53, 659-705 (1990).

[25] ed. R. C. Weast, CRC Handbook of Chemistry and Physics (CRC Press, Inc., Boca Raton, 1985).

[26] G. S. Attard and P. R. Cullum, Liquid Crystals 8, 299-309 (1990).

[27] K. Ohta, T. Watanabe, S. Tanaka, T. Fujimoto, I. Yamamoto, P. Bassoul, and N. Kucharczyk, Liquid Crystals 8, 311-330 (1990).

[28] K. Praefke, B. Kohne, B. Gundogan, D. Demus, and G. Pelzl, Liquid Crystals 7, 589-594 (1990). and references therein. 
[29] A. Khan, in Nuclear Magnetic Resonance, volume 18 (The Royal Society of Chemistry, London, 1991).

[30] ed. J. W. Emsley, Nuclear Magnetic Resonance of Liquid Crystals, NATO ASI Series C: Mathematical and Physical isiences, volume 141 (Reidel Publishing Company, Dordrecht, 1985).

[31] E. T. Samulski and R. Y. Dong, J. Chem. Phys. 77, 5090-5096 (1982).

[32] B. Janik, E. T. Samulski, and H. Toriumi, J. Phys. Chem. 91, 1842-1850 (1987).

[33] C. J. R. Counsell, J. W. Emsley, G. R. Luckhurst, and H. S. Sachdev, Mol. Phys. 63, 33-47 (1988).

[34] G. L. Hoatson, A. L. Bailey, A. J. van der Est, G. S. Bates, and E. E. Burnell, Liq. Cryst. 3, 683-694 (1988).

[35] D. J. Photinos, E. T. Samulski, and H. Toriumi, J. Phys. Chem. 94, 4694 (1990).

[36] M. Gochin. Two-Dimensional NMR of Liquids and Oriented Molecules. PhD thesis, University of California, Berkeley, 1987.

[37] S. P. Rucker. Two-Dimensional Nuclear Magnetic Resonance Studies of Molecular Structure in Liquids and Liquid Crystals. PhD thesis, University of California, Berkeley, 1991.

[38] M. Gochin, A. Pines, M. E. Rosen, S. P. Rucker, and C. Schmidt, Mol. Phys. 69, 671-695 (1990).

[39] A. Pines, S. Vega, and M. Mehring, Phys. Rev. B 18, 112 (1978).

[40] B. Janik, E. T. Samulski, and H. Toriumi, J. Phys. Chem. 91, 1842-1850 (1987).

[41] J. W. Emsley and G. R. Luckhurst, Mol. Phys. 41, 19-29 (1980).

[42] P.J. Flory, Statistical Mechanics of Chain Molecules (Wiley, New York, 1969).

[43] K. B. Wiberg and M. A. Murcko, J. Am. Chem. Soc. 10, 8029-8038 (1988).

[44] A. J. van der Est, M. Y. Kok, and E. E. Burnell, Mol. Phys. 60, 397-413 (1987).

[45] D. S. Zimmerman and E. E. Burnell, Mol. Phys. 69, 1059-1071 (1990). 
[46] G. R. Luckhurst, C. Zannoni, P. L. Nordio, and U. Segre, Mol. Phys. 30, 1345-1358 (1975).

[47] J. W. Emsley, G. R. Luckhurst, and C. P. Stockley, Proc. R. Soc. Lond. A 381, 117-138 (1982).

[48] J. P. Straley, Phys. Rev. A 10, 1881-1887 (1973).

[49] D. J. Photinos, E. T. Samulski, and H. Toriumi, Mol. Cryst. Liq. Cryst. 204, 161-176 (1991).

[50] A. M. Thayer. Pulsed Zero Field NMR of Solids and Liquid Crystals. $\mathrm{PhD}$ thesis, University of California, Berkeley, 1987.

[51] D. J. Photinos, B. J. Poliks, E. T. Samulski, A. F. Terzis, and H. Toriumi, Mol. Phys. 72, 333-344 (1991).

[52] ed. M. S. Dresselhaus, Chemical Physics of Intercalation, NATO ASI Series B: Physics, volume 148 (Plenum, New York, 1986).

[53] eds. A. P. Legrand and S. Flandrois, Chemical Physics of Intercalation", NATO ASI Series B: Physics, volume 172 (Plenum, New York, 1987).

[54] S. A. Solin and H. Zabel, Adv. Phys. 37, 87 (1988).

[55] F. Béguin, H. Estrade-Szwarckopf, J. Conard, P. Lauginie, P. Marceau, D. Guerard, and L. Facchini, Synth. Met. 7, 77 (1983).

[56] J. M. Zhang and P. C. Eklund, Synth. Met. 26, 357 (1988).

[57] M. Goldmann, A. J. Dianoux, B. Gonzalez, F. Béguin, H. Estrade-Szwarckopf, and J. Conard, Synth. Met. 23, 55 (1988).

[58] F. Béguin, R. Setton, A. Hamwi, and P. Touzain, Mat. Sci. Eng. 40, 167 (1979).

[59] N. Daumas and A. Herold, C. R. Acad. Sc. Paris 268, 373 (1969).

[60] M. F. Quinton, C. Fretigny, and A. P. Legrand, Synth. Met. 34, 569 (1989).

[61] M. F. Quinton, A. P. Legrand, L. Facchini, and F. Béguin, Synth. Met. 23, 271 (1988).

[62] F. Béguin, B. Gonzalez, M. Goldmann, and M. F. Quinton, Synth. Met. 23, 155 (1988).

[63] M. Nomine and L. Bonnetain, J. Chim. Phys. 66, 1731 (1969). 
[64] M. F. Quinton, A. P. Legrand, and F. Béguin, Synth. Met. 14, 179 (1986).

[65] M. Goldmann, J. Pannetier, F. Béguin, and B. Gonzalez, Synth. Met. 23, 133 (1988).

[66] G. R. Miller, M. J. Moran, H. A. Resing, and T. Tsang, Langmuir 2, 194 (1986).

[67] E. M. Kunoff, S. D. Goren, C. Korn, H. Riesemeier, I. Stang, and K. Luders, Phys. Rev. B 39, 6148 (1989).

[68] A. M. Panich, J. Magn. Res. 66, 9 (1986).

[69] H. Moller, W. Muller-Warmuth, E. Wein, Z. T. Lalowicz, and R. Schollhorn, J. Magn. Res. 83, 1 (1989).

[70] J. Laane in, Vibrational Spectra and Structure, volume 1 (Dekker, New York, 1972). J. R. Durig, ed.

[71] J. E. Kilpatrick, K. S. Pitzer, and R. Spitzer, J. Am. Chem. Soc. 69, 2483 (1947).

[72] W. J. Lafferty, D. W. Robinson, R. V. St. Louis, J. W. Russel, and H. L. Strauss, J. Chem. Phys. 42, 2915 (1965).

[73] K. S. Pitzer and W. E. Donath, J. Am. Chem. Soc. 81, 3213 (1959).

[74] H. J. Geise, W. J. Adams, and L. S. Bartell, Tetrahedron 25, 3045 (1969).

[75] A. L. Esteban, M. P. Galache, E. Diez, J. S. Fabian, and F. J. Bermejo, Mol. Phys. 66, 429 (1990).

[76] J. A. Greenhouse and H. L. Strauss, J. Chem. Phys. 50, 124 (1969).

[77] G. G. Engerholm, A. C. Luntz, W. D. Gwinn, and D. O. Harris, J. Chem. Phys. 50, 2446 (1969).

[78] E. Diez, A. L. Esteban, and M. Rico, J. Magn. Res. 16, 136 (1974).

[79] R. Lozach, B. Lemarie, and B. Braillon, J. Chim. Phys. 72, 873 (1975).

[80] R. Davidson and P. A. Warsop, J. Chem. Soc., Faraday Trans. 2 68, 1875 $(1972$ )

[81] L. Facchini, M. F. Quinton, A. P. Legrand, F. Béguin, and R. Setıon, Physica 99B, $525(1980)$. 
[82] D. F. Caplan. NMR Studies of the Conformation and Motion of Tetrahydrofuran in Graphite Intercalation Compounds. Master's thesis, University of California, Berkeley, 1991.

[83] Tecmag Inc., 6006 Bellaire Blvd., Houston, TX 77081.

[84] G. P. Drobny. NMR Studies of Liquid Crystals and Molecules Dissolved in Liquid Crystal Solvents. PhD thesis, University of California, Berkeley, 1982.

[85] MDB Systems Inc., 1995 N. Batavia St., Orange, CA 92613-5508.

[86] H. Y. Carr and E. M. Purcell, Phys. Rev. 94, 630 (1954).

[87] S. Meiboom and D. Gill, Rev. Sci. Instrum. 29, 688 (1958).

[88] Tecmag, Inc., Houston, TX 77081. PULSE123 User's Guide. 

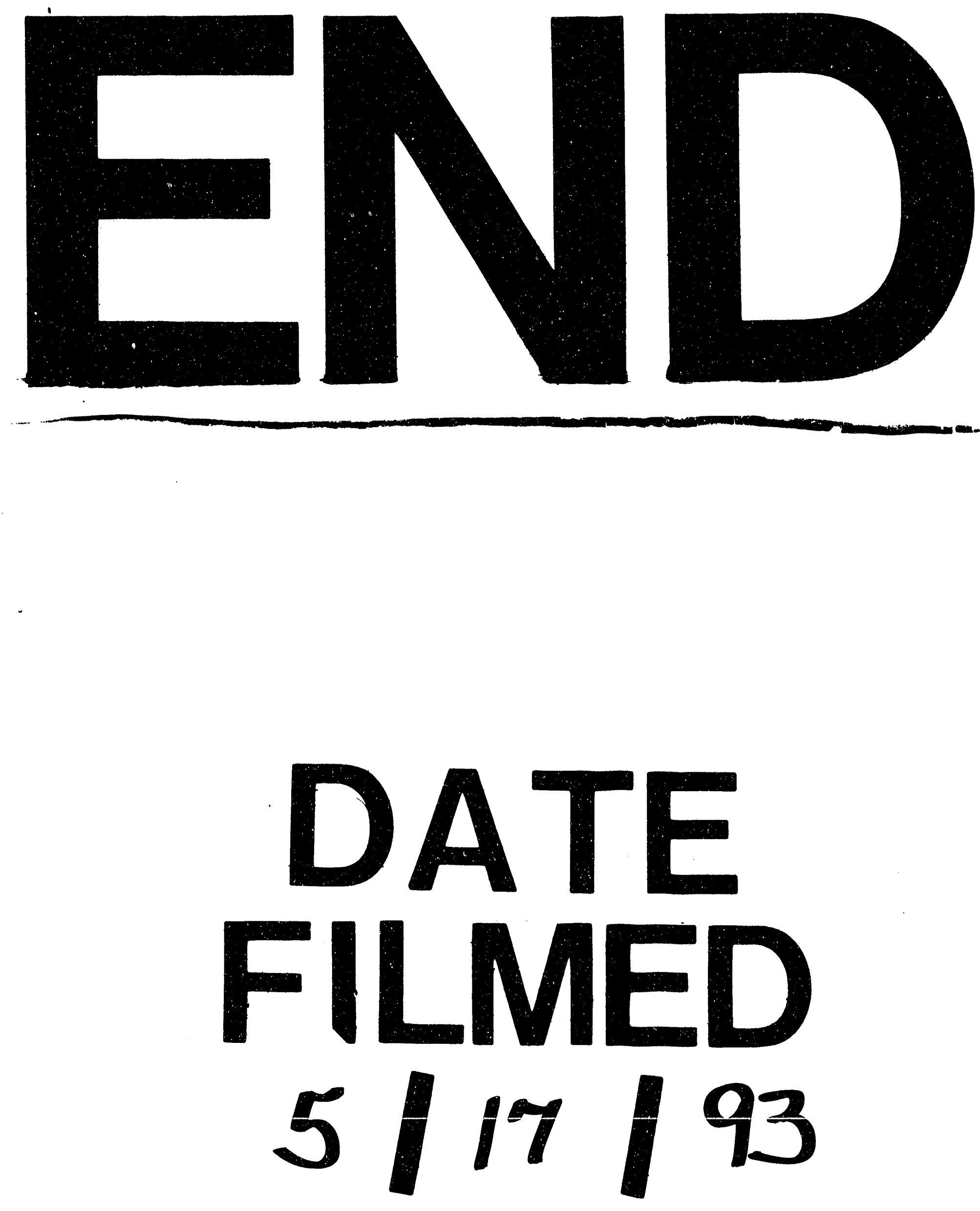

4 
